\title{
Development and Aerothermal Investigation of Integrated Combustor Vane Concept
}

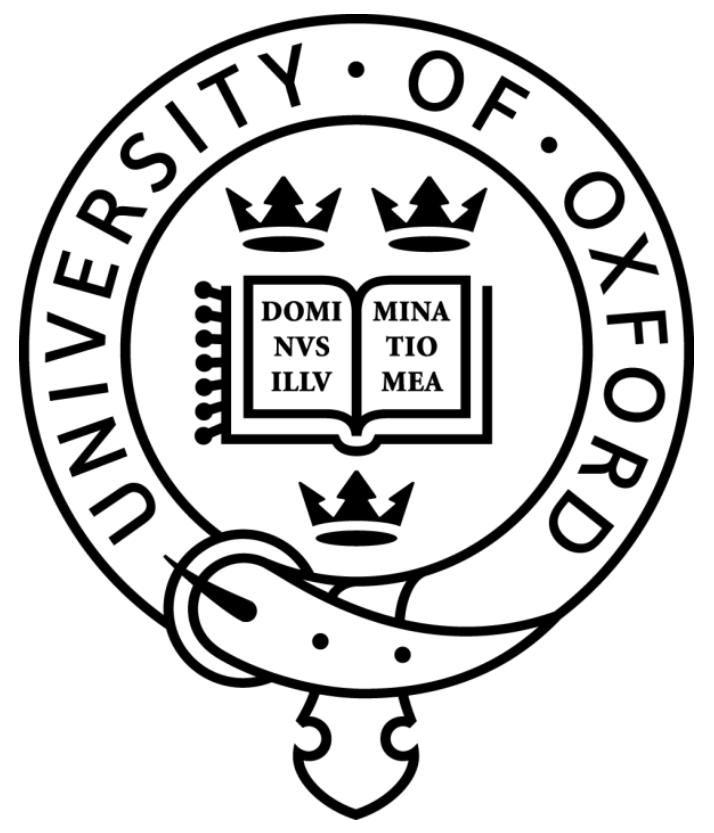

\section{Simon M. Jacobi}

Brasenose College \& Hertford College Department of Engineering Science

University of Oxford

A thesis submitted for the degree of

Doctor of Philosophy

Trinity Term 2016 


\section{Abstract}

This work focuses on the interface of combustor and high-pressure turbine of power generation gas turbines with individual can combustors. More specifically the thesis presents the development and aerothermal investigation of the integrated combustor vane concept. In this novel concept, first introduced in 2010, the conventional nozzle guide vanes (NGVs) are removed and flow turning is achieved by vanes that extend the combustor walls. The concept was developed using the inhouse CFD code TBLOCK. Aerothermal experiments were conducted using the Oxford combustor-turbine high-speed research facility. The linear cascade is comprised of two can combustor transition ducts and either four conventional vanes (CVs) or two integrated vanes (IVs). The experimental study validates the linear numerical simulations of the integrated vane development. Annular full stage simulations, used to evaluate aerodynamics and stage efficiency, confirm the trends of the linear numerical and experimental results and thus demonstrate the concept's potential for real gas turbine applications. Results show a reduction of the total pressure loss coefficient at the exit of the stator vanes by more than $25 \%$ due to a reduction in profile and endwall loss. Combined with an improved rotor performance demonstrated by unsteady stage simulations, these aerodynamic benefits result in a gain in stage efficiency of $1.5 \%$. A distinct reduction in HTC levels on vane surfaces and endwalls on the order of $40 \%-50 \%$ is observed. This is attributed to the transferral of the combustor wall's boundary layer onto the integrated vane and to the removal of the horseshoe vortex system. Furthermore it is shown that engine-realistic combustor flow with swirl leads to a slight, non-detrimental shift in the heat transfer coefficient distributions and to a less homogenous turning distribution downstream of the integrated vanes. Threedimensional vane profiling is suitable to modify the downstream turning and thus counteract the increased losses downstream of the two-dimensional integrated vanes incurred by the combustor's swirl. The experimental and numerical investigation of the 
newly developed integrated vane's endwall and leading edge cooling geometries shows a superior surface coverage of cooling effectiveness, and the cooling requirements for the first vane are expected to be halved. Moreover, by halving the number of vanes, simplifying the design and eliminating the need for vane leading edge film cooling, manufacturing and development costs can be significantly reduced. 


\section{Acknowledgements}

First and foremost I would like to thank my supervisor Dr. Budimir Rosic for his extraordinary guidance, help, and patience in conducting this work. His technical expertise and constant goodwill have been essential for the success of the project and the enjoyment along the way. Gratitude is also extended to Dr. Cosimo Mazzoni for his programming help with the in-house code TBLOCK. Furthermore I would like to thank Mitsubishi Heavy Industries for supporting this work technically and financially. Finally I am grateful to my family and friends who supported me in good times as in bad. 


\section{Table of contents}

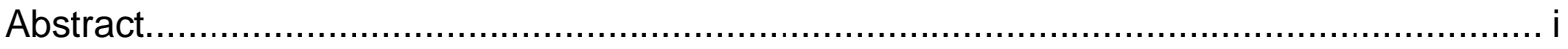

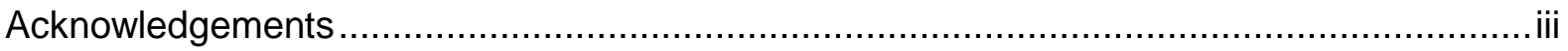

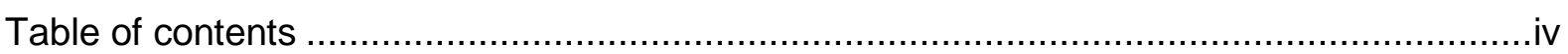

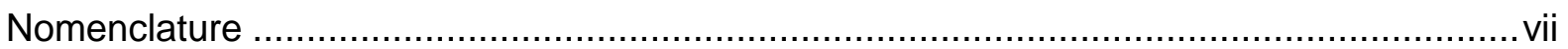

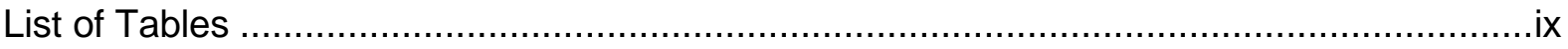

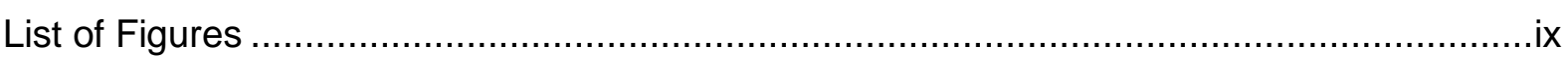

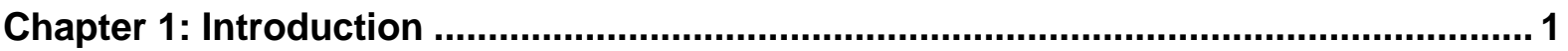

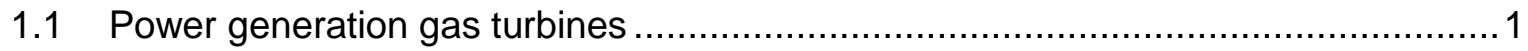

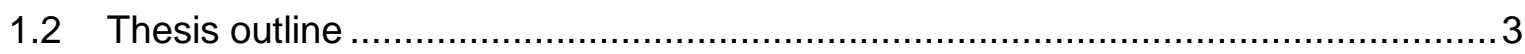

Chapter 2: The combustor-turbine aero-thermal flowfield............................................... 4

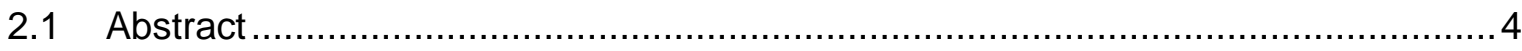

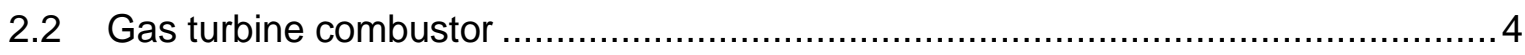

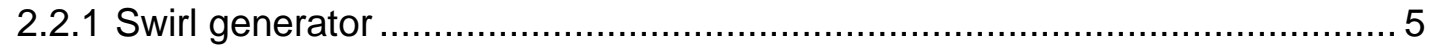

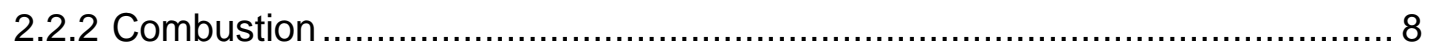

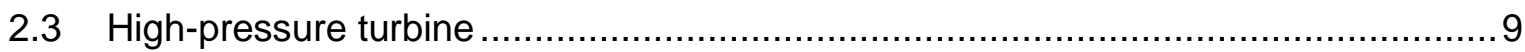

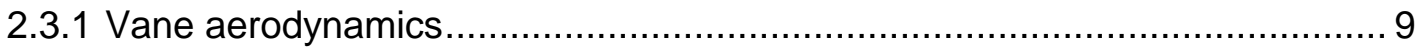

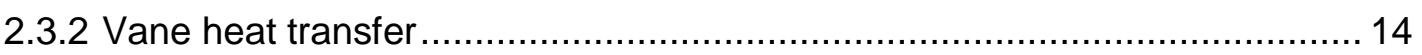

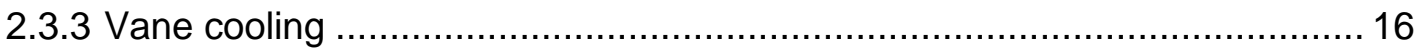

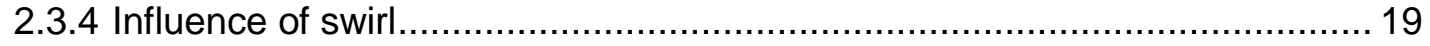

2.4 Combustor turbine interaction and integration ............................................. 20

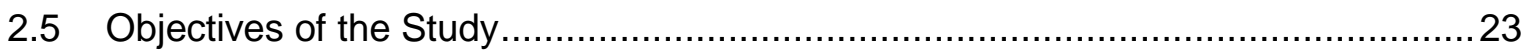

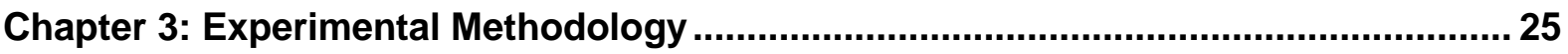

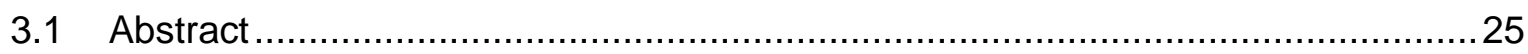

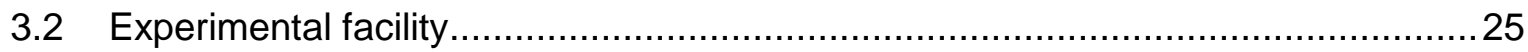

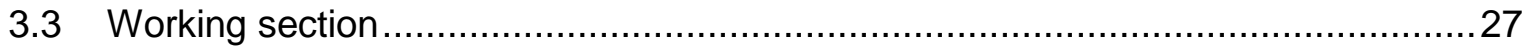

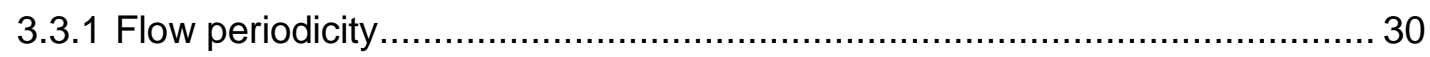

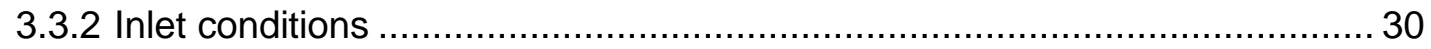




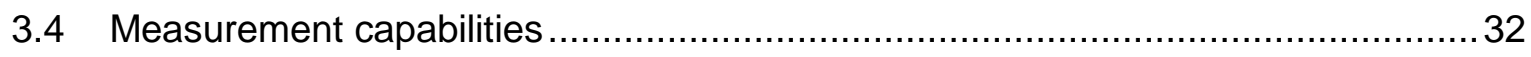

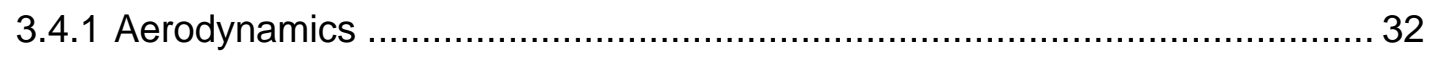

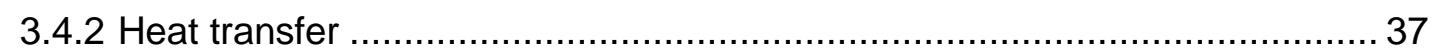

3.4.3 Summary of operating conditions …..................................................... 41

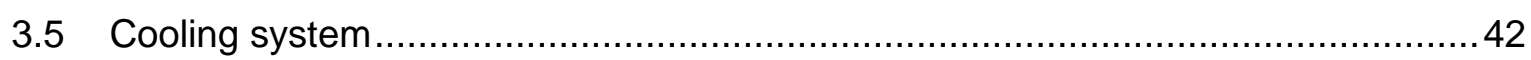

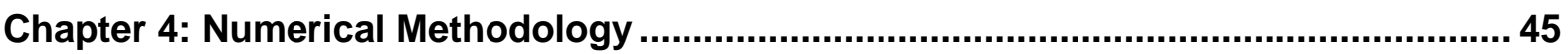

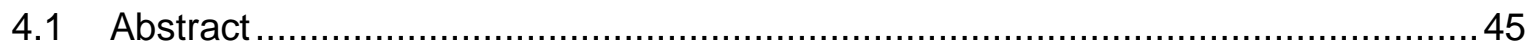

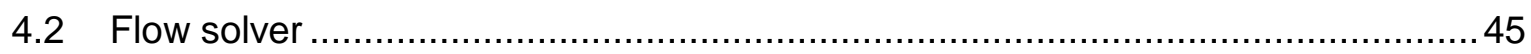

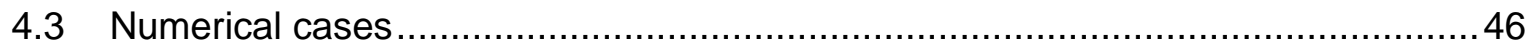

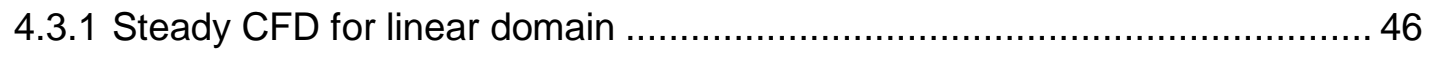

4.3.2 Unsteady CFD for annular full-stage domain ......................................... 48

Chapter 5: Design and Aerodynamic Performance of Integrated Vane ........................ 52

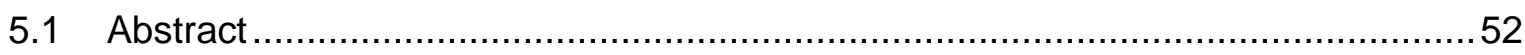

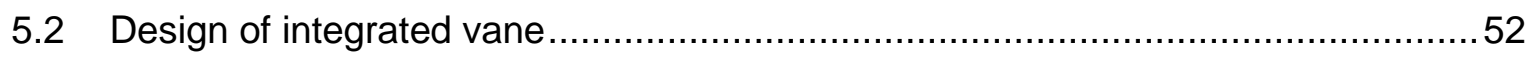

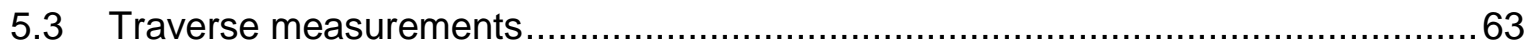

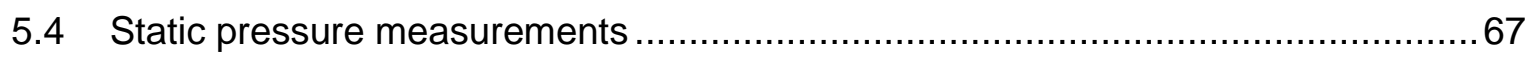

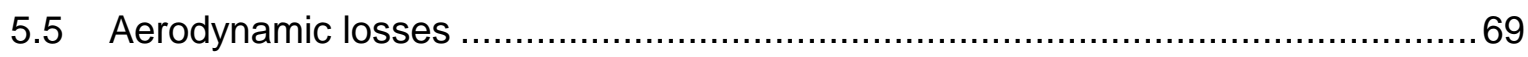

Chapter 6: Thermal Performance of Integrated Vane .................................................... 74

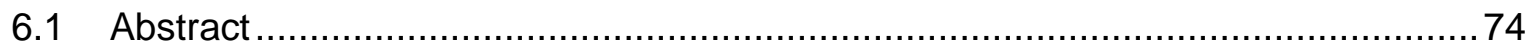

6.2 Experimental comparison of vane surface heat transfer .................................... 74

6.3 Experimental comparison of vane endwall heat transfer ...................................79

6.4 Numerical comparison of vane heat transfer.................................................. 84

6.5 Empirical correlation of vane surface heat transfer ........................................ 87

Chapter 7: Full Stage Performance of Integrated Vane ............................................... 89

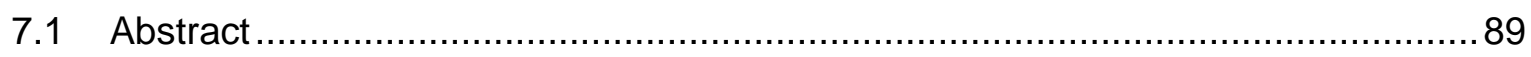

7.2 Influence of transition from linear to annular domain....................................... 89

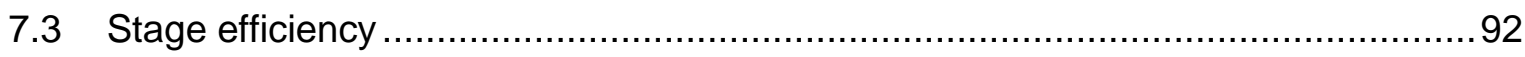

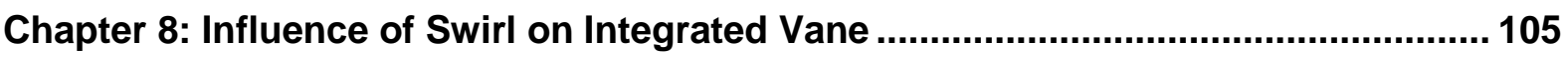

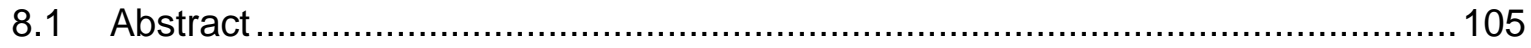




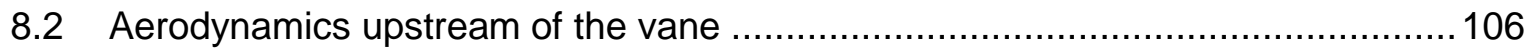

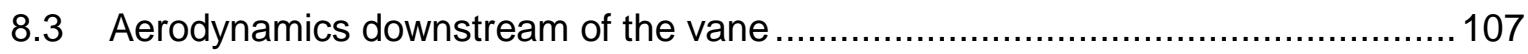

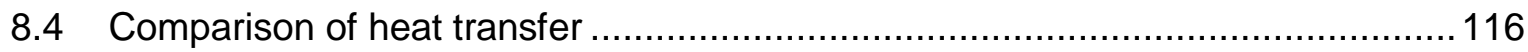

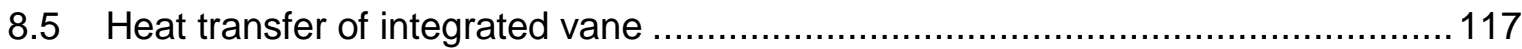

8.6 Stage performance of conventional and integrated vane ................................ 121

8.7 Integrated vane profiling for swirl ............................................................... 130

Chapter 9: Cooling Performance of Integrated Vane ..................................................... 139

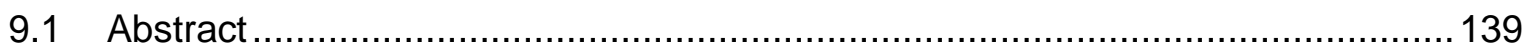

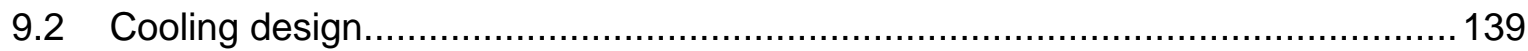

9.3 Experimental procedure and conditions...................................................... 145

9.4 Experimental and numerical cooling of conventional vane ............................... 148

9.5 Experimental and numerical cooling of integrated vane .................................. 154

9.6 Numerical comparison of cooling effectiveness with realistic endwall cooling slot

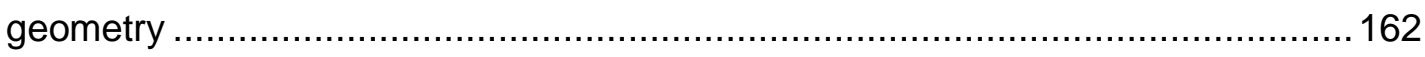

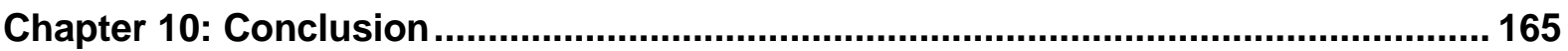

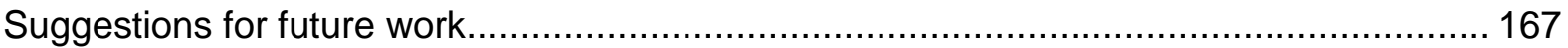

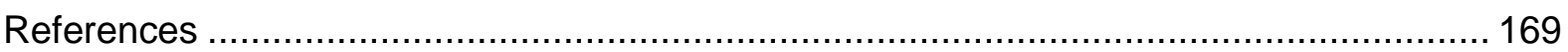




\section{Nomenclature}

\section{Abbreviations}

$\mathrm{BL}$

Boundary Layer

CV

Conventional Vane

CFD

Computational Fluid Dynamics

DC

Direct Current

DSA

Digital Sensor Array

EW

Endwall

$\mathrm{HP}$

High Pressure

HTC

Heat transfer coefficient

IP

Intermediate Pressure

IR

Infrared

IV

Integrated Vane

LE

Leading Edge

ML

Mixing Length

$\mathrm{MHI}$

Mitsubishi Heavy Industries

LPM

Lean Premixed

NGV

Nozzle Guide Vane

PS

Pressure Surface

PVC

Precessing Vortex Core

SS

Suction Surface

TE

Trailing Edge

(U)RANS

(Unsteady) Reynolds-Averaged Navier-Stokes

\section{Subscripts}

0

1

2

$\mathrm{ad}$

C

i

I

$r$
Stagnation

Inlet

Outlet

Adiabatic wall

Coolant

Initial

Lengthscale

Recovery 


$\begin{array}{ll}\text { ref } & \text { Reference } \\ \text { rel } & \text { Relative } \\ \mathrm{t} & \text { Tangential } \\ \mathrm{W} & \text { Wall } \\ \mathrm{x} & \text { Axial } \\ \infty & \text { Free-stream } \\ \theta & \text { Circumferential }\end{array}$

\section{Functions}

Entropy generation rate

$\dot{S}=\frac{T \tau \frac{d V}{d n}+\lambda\left(\frac{d T}{d n}\right)^{2}}{T^{2}}$

Dimensionless total pressure

$\overline{p_{0}}=\frac{p_{0}}{p_{0 \text { upstream }}}$

Isentropic Mach number

$M_{i s}=\sqrt{\left(\left(\frac{p_{0}}{p}\right)^{\frac{\gamma-1}{\gamma}}-1\right) \cdot \frac{2}{\gamma-1}}$

Stagnation pressure loss coefficient

$$
c_{p 0}=\frac{p_{01}-p_{0}}{p_{01}-p_{2}}
$$

Cooling effectiveness

$\eta_{\text {cooling }}=\frac{T_{\infty}-T_{a d}}{T_{\infty}-T_{c(\text { out })}}$

Entropy function

$\left(\frac{p}{p_{\text {ref }}} \cdot \frac{T}{T_{\text {ref }}}\right)^{\left(\frac{-\gamma}{\gamma-1}\right)}$

Efficiency loss

$\Delta \eta=1-\eta=\frac{T_{02} \Delta s}{\left(h_{01}-h_{02}\right)+T_{02} \Delta s_{2}}$

Swirl angle

$$
\tan ^{-1}\left(\frac{v_{t}}{v_{x}}\right)
$$

Heat flux per unit area

$\dot{q}=W /\left(m^{2}\right)$

Stanton number

$S t=\frac{N u}{R e \cdot P r}$

Heat load

$\int h t c d A$

Yaw \& pitch coefficient

$$
c_{\text {yaw }}=\frac{p_{2}-p_{4}}{p_{5}-\sum_{i=1}^{5} p_{i}} \& c_{\text {pitch }}=\frac{p_{1}-p_{3}}{p_{5}-\sum_{i=1}^{5} p_{i}}
$$




\section{List of Tables}

Table 3-1: Experimental conditions of the Oxford combustor turbine research facility ......... 42

Table 4-1: Numerical setup of full-stage simulations ................................................. 50

\section{List of Figures}

Figure 2-1: Schematic comparison of rich burn and lean premixed combustors, adapted from (Ford, Carrotte, \& Walker, 2012)

Figure 2-2: Lean premixed combustor geometry (Mandai, Matsuura, Saitoh, Tanaka, \& Akizuki, 2004) (a) and NOx generation due to combustion (b)

Figure 2-3: Flowfield downstream of swirl generator (Huang \& Yang, 2009)

Figure 2-4: Horse-shoe vortex development in first turbine vane (Sharma \& Butler, 1987).. 11

Figure 2-5: Schematic of first turbine vane cooling technology (Rolls-Royce, 2015)..... 16

Figure 2-6: Cut through an MHI F-type power generation gas turbine (a), and schematic interface of combustor and high-pressure turbine for conventional (b) and integrated (c) vane design

Figure 3-1: CAD overview (a)(b) and photo (c) of the high-speed combustor turbine research facility.

Figure 3-2: CAD (a) and photo (b) of the experimental facility's linear cascade (view from downstream), including swirlers, transition ducts and conventional vanes.

Figure 3-3: $C A D$ of the experimental facility's working section (view from downstream), including swirlers, transition ducts and integrated vanes (a) and photo of integrated vane working section (view from upstream) (b)

Figure 3-4: Measured isentropic Mach number around conventional vanes, adapted from (Luque, Kanjirakkad, Aslanidou, Lubbock, \& Rosic, 2015)

Figure 3-5: Photo of inlet cassette showing turbulence grid (a) and reversed swirler (b) ..... 31

Figure 3-6: Calibration map (a), CAD geometry (b) and photo (c) of 5-hole probe......

Figure 3-7: Photo of the experimental facility with automated 3-axis traverse mechanism and 5-hole probe.

Figure 3-8: Photo of integrated vane with pressure tappings (a) and integrated vane midspan section specifying number, position and geometry of pressure tappings (b) 36

Figure 3-9: Steel wire heater mesh of the experimental facility used for heat transfer measurements 38

Figure 3-10: Typical temperature trace of experimental run with infrared camera 38

Figure 3-11: Graph of heat flux vs. temperature of the impulse response method 40 
Figure 3-12: Schematic of vortex tube used in cooling system of the experimental facility (Airtx, 2016)

Figure 3-13: Schematic overview (top) and photo (bottom) of the cooling system for the experimental facility

Figure 4-1: Schematic of steady CFD domain for conventional (a) and integrated (b) vane design 46

Figure 4-2: Total pressure at outlet of combustor transition duct obtained from experimental traverse measurements with upstream turbulence grid (no-swirl case) . 48

Figure 4-3: Geometry of high-pressure turbine rotor used for full stage simulations 49 Figure 4-4: Annular full stage domain of conventional vane (a) integrated vane (b) and mesh at stator-rotor interface (c) 51

Figure 5-1: Predicted static pressure around integrated vane's midspan for three geometries

Figure 5-2: Predicted streamtraces on the integrated vane suction side and endwall for three geometries....

Figure 5-3: Predicted effect of three-dimensional geometry variations on dimensionless total pressure downstream of integrated vane; numbers represent averaged values

Figure 5-4: Predicted effect of three-dimensional geometry variations on turning downstream of integrated vane; numbers represent averaged values 58

Figure 5-5: Predicted effect of frontloading on the midspan static pressure distribution of the integrated vane 59

Figure 5-6: Predicted effect of frontloading on the pitchwise averaged total pressure downstream of the integrated vane

Figure 5-7: Predicted streamtraces on suction surface (top), dimensionless total pressure (middle) and yaw (bottom) distribution downstream of final integrated vane.

Figure 5-8: Aerodynamic traverse measurements showing dimensionless total pressure downstream of conventional (a) and integrated (b) vanes

Figure 5-9: Aerodynamic traverse measurements showing dimensionless total pressure downstream of conventional (a) and integrated (b) vanes

Figure 5-10: Comparison of pitchwise-averaged total pressure between traverse measurements and predictions downstream of conventional (left) and integrated (right) vanes

Figure 5-11: Comparison of pitchwise-averaged yaw between traverse measurements and predictions downstream of conventional (left) and integrated (right) vanes

Figure 5-12: Isentropic Mach number surface distribution for conventional and integrated vanes at midspan; lines and dots represent numerical and experimental results respectively 
Figure 5-13: Schematic regions of high isentropic Mach numbers and of boundary layer diffusion on suction surfaces.

Figure 5-14: Dimensionless entropy generation rate on hub endwall of conventional (a) and integrated (b) vane domain; surface streamtraces .... 71

Figure 5-15: Schematic of endwall boundary layer development for conventional (a) and integrated (b) vane design 72

Figure 5-16: Growth of total pressure loss coefficient through linear CFD domains. 73 Figure 6-1: Measured heat transfer coefficient on conventional vane (a) and integrated vane (b) suction surfaces. 75

Figure 6-2: Measured heat transfer coefficient on shielded conventional vane's (a) and integrated vane's (b) pressure surface. 77

Figure 6-3: Measured heat transfer coefficient on the conventional (a) and integrated (b) vanes leading edges

Figure 6-4: Measured heat transfer coefficient on the conventional (a) and integrated (b) vanes hub endwall (upstream view)....

Figure 6-5: Measured heat transfer coefficient on conventional vane's (a) and integrated vane's (b) hub endwall .

Figure 6-6: Axial cut showing predicted dimensionless total pressure distribution for conventional vanes (a) and integrated vane (b).....

Figure 6-7: Predicted heat transfer coefficient distribution for conventional (a) and integrated (b) vane design 84

Figure 6-8: Heat transfer coefficient levels for conventional and integrated vanes at midspan; lines and dots represent numerical and experimental results respectively. 85

Figure 6-9: Empirical correlation for the ratio of HTC between conventional and integrated vane's pressure side along conventional vane's chord.

Figure 7-1: Predicted turning (a) and dimensionless total pressure (b) contours downstream of integrated vane for annular and linear domain

Figure 7-2: Predicted heat transfer coefficient distributions of annular conventional (top) and integrated (bottom) vane domain 91

Figure 7-3: Time-averaged efficiency loss through conventional and integrated full stage domains.

Figure 7-4: Instantaneous entropy function contour of unsteady full-stage CFD for conventional (top) and integrated (bottom) vane design at midspan for no-swirl case.

Figure 7-5: Instantaneous entropy function at sliding interface for conventional vane (a) and integrated vane (b) design 95

Figure 7-6: Instantaneous relative turning at sliding interface for no-swirl case 
Figure 7-7: Conventional vane rotor instantaneous streamtraces and inlet total pressure \& relative yaw at hub (a) and casing (c) endwall, and instantaneous streamtraces on conventional vane rotor suction surfaces (b) 99

Figure 7-8: Integrated vane rotor instantaneous streamtraces and inlet total pressure \& relative yaw at hub (a) and casing (c) endwall, and instantaneous streamtraces on integrated vane rotor suction surfaces (b). 100

Figure 7-9: Axial cut of instantaneous entropy function downstream of rotor for conventional (top) and integrated (bottom) vane design for a no-swirl case

Figure 7-10: Time- and pitchwise-averaged entropy function at rotor inlet and outlet for a noswirl case. 104

Figure 8-1: Measured dimensionless total pressure (top), yaw (middle) and pitch (bottom) distributions downstream of the transition duct (vanes not present) for a swirl (a) and a reversed swirl (b) case

Figure 8-2: Traverse measurements downstream of integrated vane showing dimensionless total pressure distributions for a no-swirl (top), swirl (middle) and reversed swirl (bottom) setup 108

Figure 8-3: Traverse measurements downstream of integrated vane showing yaw distributions for a no-swirl (top), swirl (middle) and reversed swirl (bottom) setup 109 Figure 8-4: Predicted turning for a no-swirl (a), swirl (b) and reversed swirl (c) case 110 Figure 8-5: Pitchwise averaged dimensionless total pressure (left) and yaw (right) downstream of the integrated vane for a no-swirl, swirl and reversed swirl scenario; dots and lines represent experiments and simulations respectively

Figure 8-6: Predicted dimensionless total pressure downstream of the integrated vane with swirl (a) and reversed swirl (b); axial cut of static pressure; iso-surface of total pressure; streamtraces on suction surface

Figure 8-7: Traverse measurements downstream of conventional vanes showing dimensionless total pressure (top) and yaw (bottom) distributions for a swirl setup 114 Figure 8-8: Pitchwise averaged dimensionless total pressure (left) and yaw (right) obtained from experimental traverse measurements for the swirl and no-swirl cases. 115 Figure 8-9: Measured heat transfer coefficient on conventional vane (a) and integrated vane (b) suction surfaces with inlet swirl. 116 Figure 8-10: Measured HTC contour and predicted isentropic Mach number lines (top) and predicted HTC contour (bottom) on integrated vane's suction surface with swirl (left) and reversed swirl (right).

Figure 8-11: Measured HTC contour and predicted isentropic Mach number lines on integrated vane's pressure surface with swirl (left) and reversed swirl (right) 119 
Figure 8-12: Measured HTC on integrated vane's endwall with swirl (a) and reversed swirl (b) 120

Figure 8-13: Influence of swirl on stage efficiency loss for conventional and integrated vanes

Figure 8-14: Instantaneous entropy function at midspan (a), and axial cut upstream (b) downstream (c) of rotor for conventional vane stage simulation with swirl.

Figure 8-15: Time- and pitchwise-averaged turning at the sliding interface for a no-swirl (solid line) and swirl (dotted line) case

Figure 8-16: Instantaneous streamtraces on integrated vane rotor for a no-swirl (a) and a swirl (b) case. 126

Figure 8-17: Instantaneous entropy function at 33\% span (a), and axial cut upstream (b) and downstream (c) of rotor for integrated vane stage simulation with swirl. 128

Figure 8-18: Time- and pitchwise-averaged entropy function at rotor inlet and outlet for the swirl case.

Figure 8-19: Schematic of three-dimensional vane profiling with 'original' two-dimensional integrated (a) and 'new' profiled integrated vane (b) 131

Figure 8-20: Predicted turning downstream of two- (a) and three- (b) dimensional integrated vane, and pitchwise averaged yaw (c) for steady linear simulation.

Figure 8-21: Predicted dimensionless total pressure distribution downstream of two- (a) and three- (b) dimensional integrated vane, and pitchwise averaged dimensionless total pressure (c) for steady linear simulation 133

Figure 8-22: Predicted heat transfer coefficient distribution on two- (a) and three- (b) dimensional integrated vane suction surface for steady linear simulation. 134

Figure 8-23: Predicted effect of integrated vane profiling on stage efficiency loss 135 Figure 8-24: Instantaneous entropy function at 33\% span (top) and axial cut downstream of rotor (bottom) for profiled integrated vane stage simulation with swirl 136 Figure 8-25: Instantaneous streamtraces on integrated (a) and profiled integrated (b) vane rotor for the swirl case.

Figure 8-26: Time- and pitchwise-averaged entropy function at rotor inlet and outlet for the swirl case.

Figure 9-1: Schematic of integrated vane's endwall and leading edge cooling geometry and mesh

Figure 9-2: Predicted static pressure contour of integrated vane leading edge cooling design and seal at midspan; velocity vectors.

Figure 9-3: Predicted stagnation pressure in feeding plenum for leading edge cooling design 
Figure 9-4: Geometry of cooling box used for endwall cooling (top), and slice of predicted

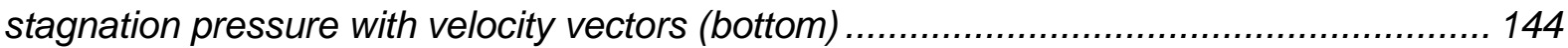

Figure 9-5: Photo of cooling box for leading edge cooling (a) and endwall cooling (b)....... 145 Figure 9-6: Measured temperatures during a cooling run on the experimental facility ....... 146 Figure 9-7: Measured pressures in the working section during an experimental cooling run

Figure 9-8: Predicted cooling effectiveness of conventional vane design with endwall cooling 149

Figure 9-9: Measured cooling effectiveness on conventional vane hub endwall with endwall cooling for a suction side view. 150

Figure 9-10: Measured cooling effectiveness on conventional vane hub endwall with endwall cooling for a pressure side view

Figure 9-11: Measured cooling effectiveness on conventional vane hub endwall (looking from downstream) with endwall cooling.

Figure 9-12: Measured cooling effectiveness on conventional vane suction surfaces with endwall cooling 153

Figure 9-13: Predicted cooling effectiveness of integrated vane design with leading edge (a) and endwall (b) cooling 155

Figure 9-14: Measured cooling effectiveness on integrated vane leading edge with leading edge cooling 156

Figure 9-15: Measured cooling effectiveness on integrated vane suction surface with leading edge cooling 157

Figure 9-16: Measured cooling effectiveness on integrated vane hub endwall with endwall cooling

Figure 9-17: Measured cooling effectiveness on integrated vane hub endwall and suction surface with endwall cooling. 159

Figure 9-18: Measured cooling effectiveness on integrated vane suction surface with endwall cooling

Figure 9-19: Dimensionless static pressure on hub endwall of conventional and integrated vane in the location of the cooling slot obtained from steady CFD simulations without cooling 161

Figure 9-20: Schematic of realistic geometry and mesh of endwall cooling slots 163 Figure 9-21: Predicted endwall cooling effectiveness of conventional vane (left), and endwall \& leading edge cooling effectiveness of integrated vane (right)..... 164 


\section{Chapter 1: Introduction}

\subsection{Power generation gas turbines}

The quest for sustainability has been leading to a transition in power generation. Pollutive energy sources such as coal are continuously phased out to be replaced with renewable energy sources such as wind and the sun. While costs of these renewables are decreasing due to economies of scale and increases in efficiency, unpredictability of their power supply still represents an impediment: matching of energy supply (the wind does not always blow, the sun does not always shine) and demand (low at night, high in the mornings and evenings) is difficult. Despite significant research on energy storage, current technologies are not capable to handle this task at the scales needed. This is where gas turbine power generation comes into play. It allows very flexible operation (turning it on and off when needed) at a relatively low cost and low emission footprint. As a result, predictions show that the share of gas turbines in the power generation mix (22\% in 2011 (IEA, 2011)) is likely to increase for the next decades to come. This puts significant emphasis on further improving gas turbine efficiency and reducing emissions. A further advantage lies in the easy scaling of gas turbine size and power output. A suitable gas turbine can thus be installed easily where energy is needed. This not only helps when electricity transport is difficult (such as in remote areas that are not or not well connected to the grid) but also allows for autonomous operation (independence of grid allows to avoid power outages). The vast applicability of gas turbines puts it in direct competition with other power technologies. One of the most driving parameters in this competition is cost, not only in operation (defined by efficiency) and maintenance (defined by part life cycle) but especially by acquisition. This can be 
achieved by simplifying the design in order to employ only the essential parts and minimize the development time (and thus cost).

Gas turbine research and development has been an ongoing process for over a century. Particular focus of power generation gas turbines is the thermal efficiency $\eta_{t h}=\frac{\dot{W}}{\dot{Q}}$. It is defined as the ratio of useful work $(\dot{W})$ to heat $(\dot{Q})$ supplied in the combustor and it determines the fuel consumption. Increasing peak gas temperatures and reducing coolant requirements are paramount for optimising gas turbine performance and maximising efficiency. Impressive achievements have been made so far: MHI's J-type, for instance, is a $1600^{\circ} \mathrm{C}$ class gas turbine with a combined cycle efficiency of $61.5 \%$ (Hada, Yuri, Masada, Ito, \& Tsukagoshi, 2012) and targets for temperature as well as efficiency are set even higher for the next generation gas turbine. This has been made possible by various core technologies such as employing advanced materials (e.g. super-alloy), single crystal casting, thermal barrier coating, and sophisticated internal and external cooling strategies. Despite the abundance of research on a variety of topics such as these, it seems as though bottom-line progress has become rather incremental. In order to achieve further significant improvements, a step change in power generation gas turbine technology is needed. And for this to happen, innovation should supplement convention. The integrated combustor vane concept represents an unconventional approach. It suggests the removal of the high-pressure turbine's nozzle guide vanes and the achievement of the necessary flow turning with novel vanes that effectively prolong the combustor walls. In order to deem it a viable technology, its benefits should outweigh its drawbacks compared to the datum design. This thesis thus aims to analyse the four most crucial aspects: aerodynamic performance, thermal performance, stage efficiency and cooling performance. Further important aspects such as manufacturability and costs shall also be briefly discussed. 


\subsection{Thesis outline}

This thesis consists of 9 chapters which will be briefly outlined at this point. Chapter 2 gives an overview of the aerothermal flowfield at the combustor turbine interface and of the relevant research up-to-date. Chapter 3 introduces the experimental facility, its working principle and the measurement capabilities. Chapter 4 presents the numerical methodology comprising the in-house flow solver TBLOCK, the computational domains and the boundary conditions. Chapter 5 describes the development of the integrated vane's profile based on two parametric numerical studies. The predictions are verified with experimental measurements, and the aerodynamic benefits compared to a conventional vane design are highlighted and explained. Chapter 6 focuses on the investigation of the integrated vane's thermal performance. Experimental measurements show thermal benefits of the new concept. These are validated with numerical simulations - which also help to explain these benefits - and an empirical correlation. Chapter 7 shows the predicted fullstage performance of the integrated vane design with a considerable efficiency advantage over the datum case. Chapter 8 investigates the effect of combustor swirl on the integrated vane's aerodynamic and thermal performance. Experimental measurements validate the numerical predictions and show considerable variations in downstream turning. It is furthermore shown that integrated vane profiling is suitable to homogenize the flow non-uniformities caused by swirl and thus further improve stage efficiency. Finally, the integrated vane's cooling characteristics are presented in Chapter 9. The predictions are validated with experiments, and the concept's benefits over the conventional vane design are highlighted. 


\section{Chapter 2: The combustor-turbine aero- thermal flowfield}

\subsection{Abstract}

This chapter will discuss the aerothermal flowfield at the combustor-turbine interface and present relevant research to date. Firstly, the gas turbine combustor will be introduced, including the importance of the combustion process and the aerodynamic effects of its swirl-generators. Subsequent discussions concentrate on the most relevant aspects of the high-pressure turbine: Aerodynamics, heat transfer, cooling, and the influence of swirl. Finally, the importance of coupling the combustor with the turbine in experiments and simulations is highlighted.

\subsection{Gas turbine combustor}

Gas turbine combustors add energy (in the form of heat) to the air stream exiting the compressor in order to power the turbine (which drives the compressor and potentially the fan) and to provide rotational energy (power generation) or propulsive thrust (aviation). A historical overview of gas turbine combustor design, dating back to 1940s, is given by Lefebvre and Ballal (2010). Nowadays, Lean-Premixed (LPM) combustors are broadly used in industry. Stringent regulations are set for the goal of reducing $\mathrm{NO}_{\mathrm{x}}$ emissions (levels below 9ppm already) due to their detrimental effects including acid rain, photochemical smog, depletion of the ozone layer and global warming (Correa, 1998). This has raised public concern and been the driving parameter in the development of NOx-emissions control techniques and combustion technology (Bowman, 1992). LPM combustion promises to offer the highest potential for $\mathrm{NO}_{x}$ reduction (Lazik, Doerr, Bake, Bank, \& Rackwitz, 2008). This is achieved by 
LPM combustor's architecture, in which different configurations of swirl injectors generate a lean mixture of fuel and air prior to combustion. Figure 2-1 shows that rich burn combustors use only $30 \%$ of the air flow for combustion and $70 \%$ for quenching, while lean premixed combustors use $70 \%$ of the air flow for the combustion and $30 \%$ for cooling (Ford, Carrotte, \& Walker, 2012). This results in lower temperatures and thus lower $\mathrm{NO}_{x}$ emissions compared to conventional rich-burn combustors.
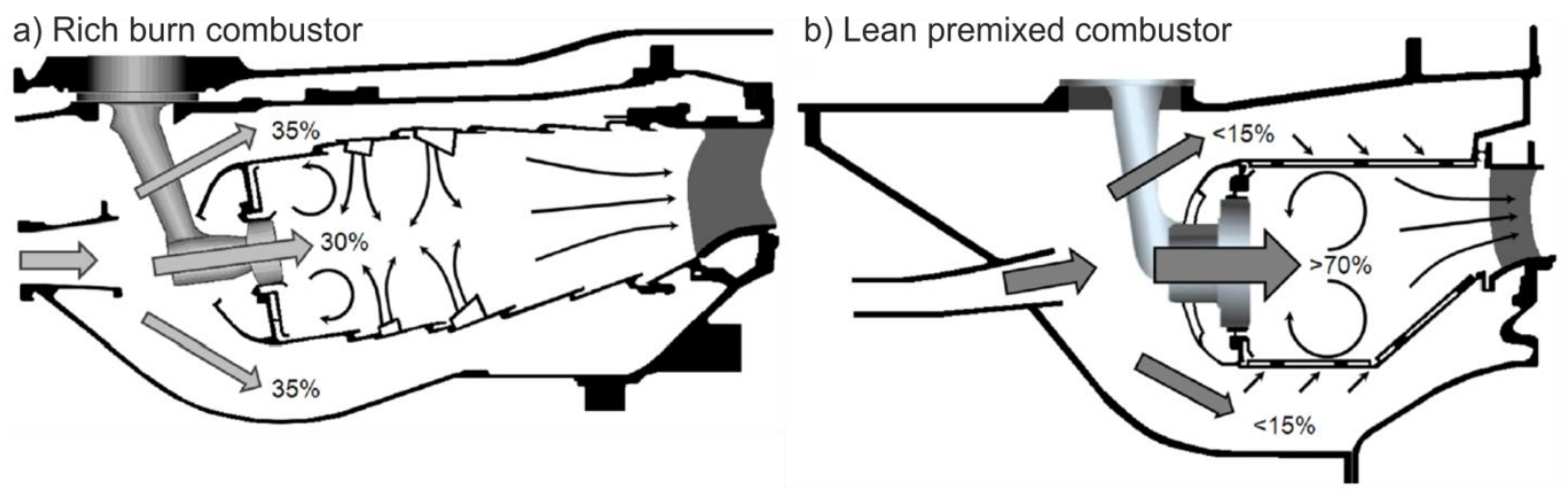

Figure 2-1: Schematic comparison of rich burn and lean premixed combustors, adapted from (Ford, Carrotte, \& Walker, 2012)

\subsubsection{Swirl generator}

Swirl generators are used in gas turbine combustors to achieve effective combustion through mixing of fuel and air. They consist of one pilot swirler in the centre and a number of main swirlers situated around the pilot swirler. The pilot swirler produces a diffusion flame to ensure steady and safe operation, while the main swirlers produce a premixed flame for a more efficient combustion process with low emissions. This can be seen in Figure 2-2 (right), which also shows a typical geometry of such a lean premixed combustor (left). This can-combustor is based on the United States patent US6701713 B2 published by Mandai et al. (2004) and granted to Mitsubishi Heavy Industries Ltd. 
a) Lean-premixed combustor geometry

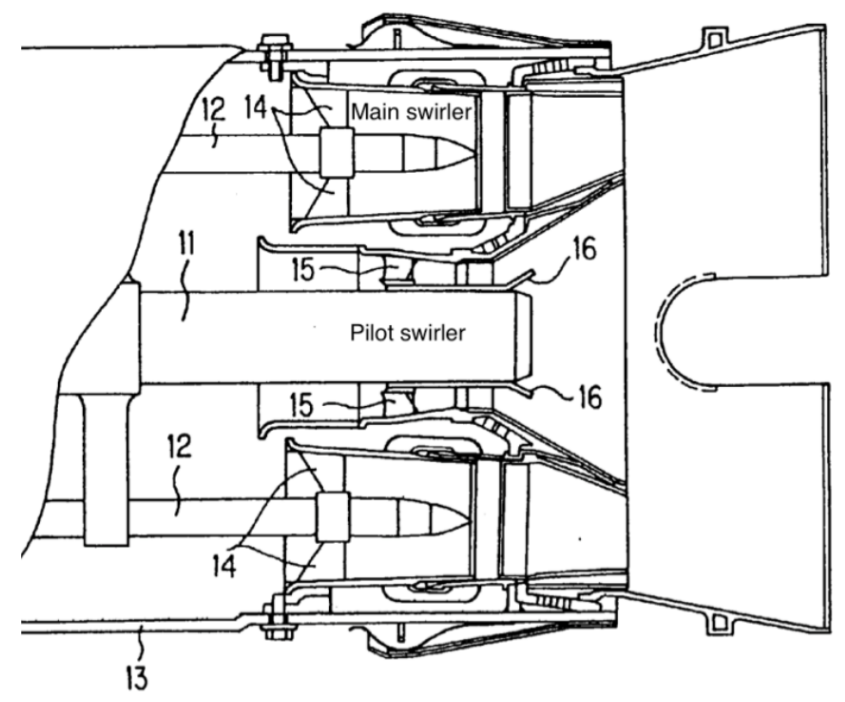

b) Combustion $\mathrm{NO}_{x}$ generation

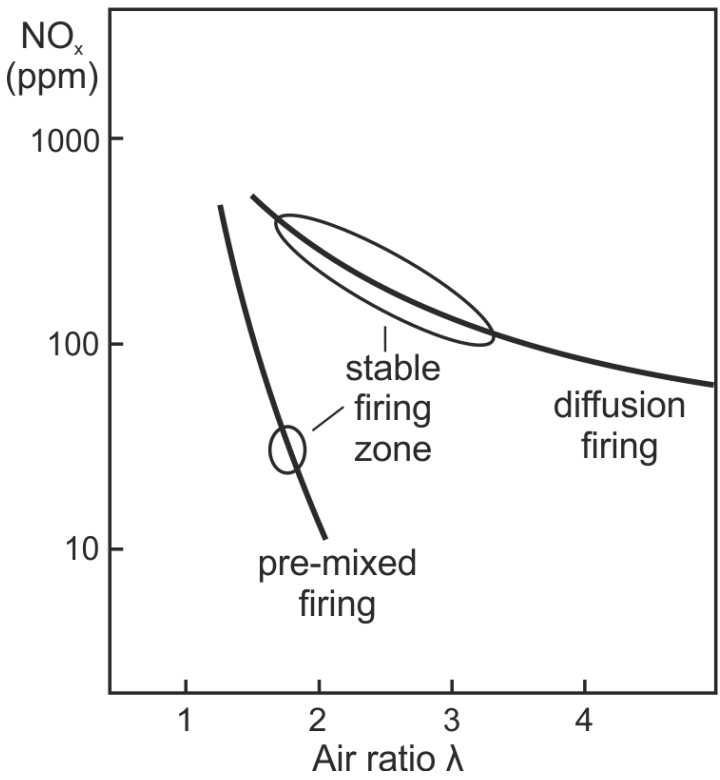

Figure 2-2: Lean premixed combustor geometry (Mandai, Matsuura, Saitoh, Tanaka, \& Akizuki, 2004) (a) and NOx generation due to combustion (b)

The swirl generators greatly influence the aerodynamic behaviour of fluids within LPM combustors, and an overview of different configurations and designs can be found in (Huang \& Yang, 2009). The effective mixing is achieved through strong shear regions and high turbulence (Lefebvre A. , 1999) (Alekseenko, Kuibin, \& Okulov, 2007). The fluid exiting the swirl generators typically shows three flow structures (Huang \& Yang, 2009), which can be schematically seen in Figure 2-3.

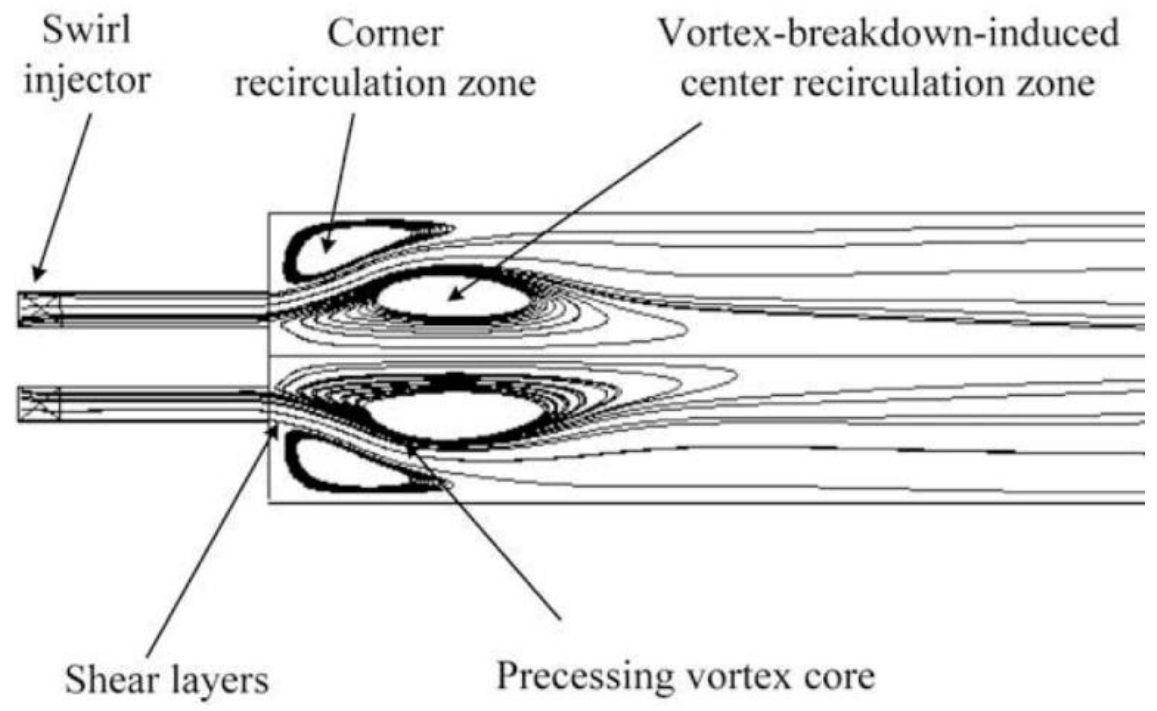

Figure 2-3: Flowfield downstream of swirl generator (Huang \& Yang, 2009) 
Firstly, a recirculation zone develops in the centre due to vortex breakdown which causes an adverse pressure gradient. The momentum balance:

$$
\frac{d p}{d r}=\frac{\rho u_{\theta}}{r}
$$

shows that high circumferential velocities $u_{\theta}$ (caused by strong swirl) lead to a radial static pressure gradient which in turn can cause a flow reversal downstream of the swirler (Xiyun, Wang, Sung, Hsieh, \& Yang, 2005). This is beneficial in combustors as it increases residence times and thus leads to a more complete combustion process. Secondly, corner recirculation zones establish when the main flow exiting the swirl generators encounters the ambient fluid, leading to shear layers due to velocity gradients and thus to vortex structures. Thirdly, a Precessing Vortex Core (PVC) is often observed at a certain frequency around the former recirculation zone (Anacleto, Fernandes, Heitor, \& Shtork, 2003); the frequency of precession depends on the burner/swirl configuration and increases linearly with flow rate (Syred, 2006). There are also a few studies which quantify the processes occurring under PVC driven oscillations for representative but very different conditions, using a variety of experimental techniques (Schildmacher \& Koch, 2005) (Dawson, RodriquezMartinez, Syred, \& O'Doherty, 2006) and numerical approaches (Turrell, Stopford, Syed, \& Buchanan, 2004) (Schluter, Schonfeld, Poinsot, Krebs, \& Hoffman, 2001). This precessing vortex core has also been shown to have a significant effect on the vanes unsteady aerothermal performance (Jacobi, Mazzoni, Chana, \& Rosic, 2017). It is critical to have a good understanding of the flowfield in the combustor in order to ensure combustion and turbine performance, operating reliability and structural integrity. 


\subsubsection{Combustion}

The chemical reactions occurring during combustion influence the flowfield within the combustor downstream of the swirl-injectors (Cameron, Brouwer, Wood, \& Samuelsen, 1989) (Goebel, Abuaf, Lovett, \& Lee, 1993). It is reported that the precessing vortex core and vortex breakdown will likely differ between a reacting and a non-reacting scenario for high swirl and diffusive flows (Roux, Lartigue, \& Poinsot, 2005) (Selle, Benoit, Poinsot, Nicoud, \& Krebs, 2006) (Wankhede, Bressloff, Keane, Caracciolo, \& Zedda, 2010) (O'Connor \& Lieuwen, 2011). The oscillations of the PVC can potentially also be suppressed by combustion, as illustrated by Selle et al. (Selle, et al., 2004). This difference is due to the instability caused by heat-release in the reacting case. This lack of heat-release in the non-reactive case imposes challenges for the goal of achieving engine representative swirl- and temperature-distributions as target combustor outlet profiles simultaneously. Nonetheless, chemical reactions are omitted in many studies for reasons of simplification (Wang, Bai, \& Wessman, 2004) (Wang, Yang, Hsieh, \& Mongiah, 2007) (Hall, Chana, \& Povey, 2014).

A comprehensive overview of the research on unsteady pressure, temperature and turbulence in combustors is given by Lubbock (2013). Current research suggests that exit turbulence is primarily a result of combustor geometry, swirl and secondary flows (Moss, 1992), and the combustion process does not appear to have a significant impact (Zimmerman, 1979) (Barringer, Richard, Walter, Stitzel, \& Thole, 2002). The level of swirl is however affected by combustion, with which it appears to decrease (Cameron, Brouwer, Wood, \& Samuelsen, 1989). Turbulence intensity may therefore be reduced by combustion depending on the level of swirl responsible for the turbulence. 


\subsection{High-pressure turbine}

The high pressure and temperature flow from the combustor enters the turbine. The fluid's energy is partially converted into mechanical energy by the rotor. "The first task of the turbine system is [however] survival" (Rolls-Royce, 2015). The complex flowfield produced by LPM combustors leads to aerothermal challenges for the turbine. The first vane is subject to high and non-uniform turbulence intensities and lengthscales (as high as 35\% (of $\overline{v_{x}}$ ) and $25 \%$ (of $c_{x}$ ) respectively (Cha, Hong, Ireland, Denman, \& Savarianandam, 2012)), and non-uniform temperature, pressure and velocity profiles (e.g. PVC, residual swirl, boundary layers) which significantly affect the aerodynamic performance, heat transfer, and film cooling within the turbine. Predicting the heat transfer and cooling of aerofoil endwalls has become particularly critical. This is due to its large surface area (in today's low aspect ratio and low solidity turbine designs) and due to the increased temperatures at the endwalls caused by a flatter temperature profile (in modern lean burn combustion systems).

\subsubsection{Vane aerodynamics}

The fluid emanating from the combustor is expanded through the stationary nozzle guide vanes and turned from approximately $0^{\circ}$ to $70^{\circ}$. Closer to the endwalls, the flow is however subject to secondary flows.

\section{Secondary flows}

The existence of velocity, temperature and pressure gradients at the endwall leads to secondary flows such as the horse-shoe vortex which are difficult to predict but have a strong influence on turbine efficiency, endwall cooling and heat transfer (Thole, 2006). Secondary flow can be defined as the velocity component normal to the 'primary' direction: 


$$
\overline{v_{\text {sec }}}=\bar{v} \times \overline{\boldsymbol{e}_{p}}
$$

with $\overline{e_{p}}$ being the unit vector in the 'primary' direction. Langston (1980), Sharma and Butler (1987), and Goldstein and Spores (1988) illustrated the three main representative models of the secondary flow development on the endwall. When the upstream boundary layer approaches the vane stagnation pressure region, the low momentum fluid close to the endwall is turned faster than the higher momentum fluid further from the endwall. The boundary layer thus rolls up into a horse-shoe vortex. The pressure side leg of the horse-shoe vortex is convected to the adjacent vanes suction side due to the passage's pitchwise pressure gradient. This passage vortex 'collects' the inlet boundary layer (and sweeps away potential upstream endwall film coolant (Friedrichs, Hodson, \& Dawes, 1999)), and finally interacts with the suction side leg of the horse-shoe vortex. A schematic of this horse-shoe vortex development can be seen in Figure 2-4. It should be noted however that this is a simplified schematic. Since the flowfield in turbomachines is highly unsteady, so are its secondary flows. Jacobi et al. (2017) for instance observed large pitchwise fluctuations of the passage vortex over time, contrary to the steady convection suggested in the textbook example.

Secondary flow is an inviscid phenomenon and its mechanisms are described by Came and Marsh (1974). As such it does not necessarily result in loss but usually does. Although the loss associated with endwall flows has been studied for a long time, full physical understanding is still limited. A comprehensive overview of the research on secondary flows in axial turbines is given by Pullan and Denton (2012), Sieverding (1985) and Gregory-Smith et al. (1988). 


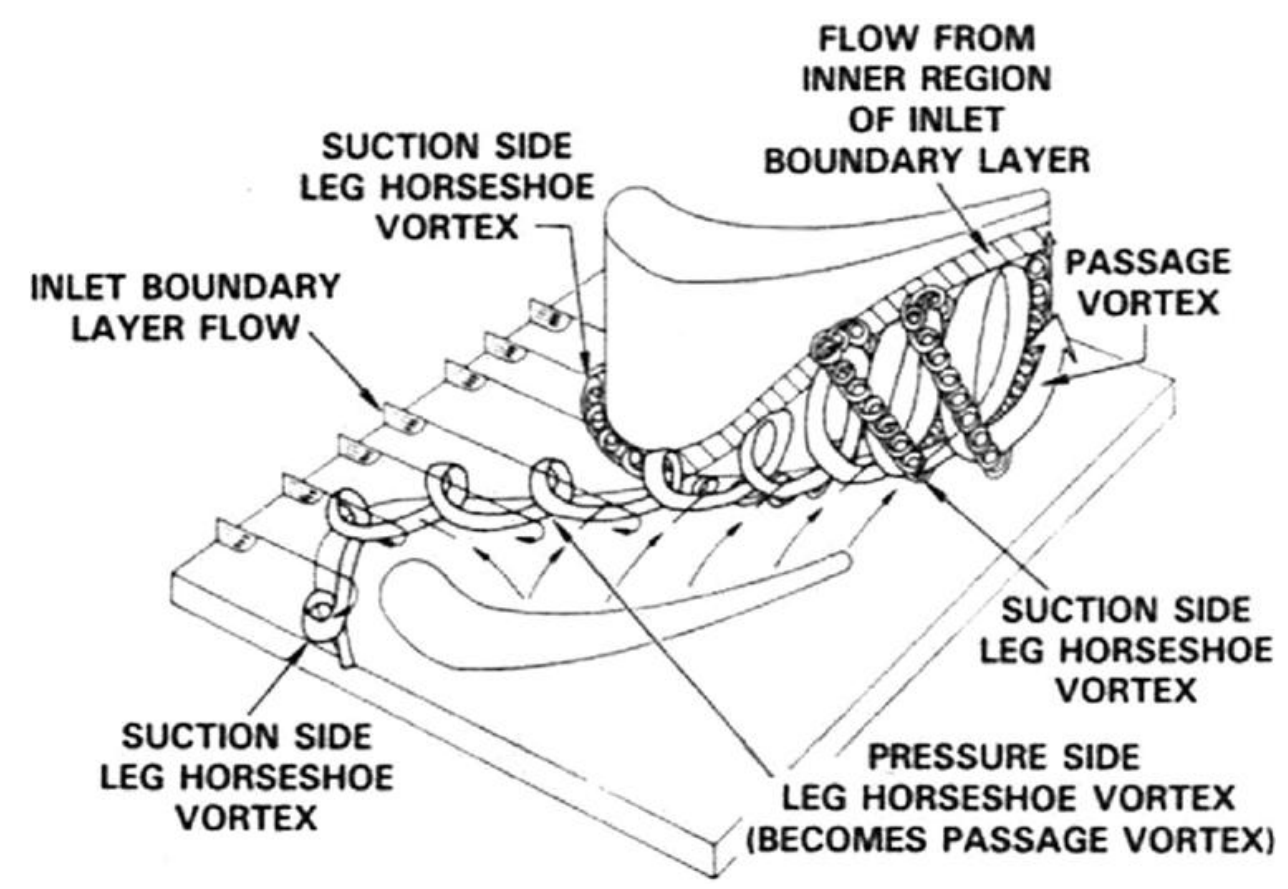

Figure 2-4: Horse-shoe vortex development in first turbine vane (Sharma \& Butler, 1987)

\section{Losses}

A thorough description of all fundamental loss mechanisms in turbomachines is given by Denton (1993). Entropy increase is an accurate measure of loss and is caused by shock waves, viscous effects during mixing and in boundary layers, and heat transfer across temperature differences. The loss can be categorized into profile loss, leakage loss and endwall loss although it is difficult to completely separate them. Each of them usually accounts for $1 / 3$ of the total turbine efficiency loss. Profile loss is generated in the boundary layer on the blade away from the endwall and includes the trailing edge loss. Leakage loss comprises the loss generated due to the rotor clearance at the casing and the stator clearance at the hub. Endwall loss constitutes the loss not accounted for by profile and leakage loss and is usually a result of secondary flows.

While entropy generation is the most accurate description of loss, loss coefficients are often used. This is because while entropy cannot be directly measured, loss 
coefficients can be easily calculated from test data. In this work, the stagnation pressure loss coefficient will be used. It is defined as:

$$
c_{p 0}=\frac{p_{01}-p_{0}}{p_{01}-p_{2}}
$$

Another measure of loss creation used in this study is the entropy generation rate per unit volume of fluid, which helps to pinpoint the loss origin in the CFD-simulations. It is defined as (Denton J., 1993):

$$
\dot{S}=\frac{T \tau \frac{d V}{d n}+\lambda\left(\frac{d T}{d n}\right)^{2}}{T^{2}}
$$

Furthermore, the turbine stage efficiency is defined as (Denton J. , 1993):

$$
\eta_{t} \approx \frac{h_{1}-h_{2}}{h_{1}-h_{2}+T_{2}\left(s_{2}-s_{1}\right)}
$$

Differences between stagnation and static conditions are neglected for this equation, with no external heat transfer. This thesis will compare the growth of entropy through the stage for various computational domains and inlet conditions using the lost efficiency (Denton J. , 1993):

$$
\Delta \eta \approx \frac{T_{02} \Delta s}{h_{01}-h_{02}+T_{02} \Delta s_{2}}
$$

\section{Vane design}

The aim of the first vane is to smoothly guide the flow into the rotor. This vane turning should involve minimal loss and result in homogeneous downstream distributions. This is achieved through varying the vane loading (e.g. front-/aft-loading) and using vane profiling techniques (e.g. sweep and lean). Sweep, which induces stream surface twist, can be used to alter the loading at leading and trailing edge near the endwalls (Denton J. , 1994). Blade lean, i.e. non-radial stacking that causes a 
change in stream surface thickness and twist, is mostly used to vary the loading distribution across the span and to reduce the endwall loss.

Studies by Pullan et al. (2006) on a low aspect ratio nozzle guide vane showed that the influence of secondary flows can be reduced by aft-loading the vane, thus resulting in a considerable stage efficiency gain (in this case of $0.5 \%$ over the datum case). Further theoretical, experimental and numerical investigations by Pullan and Harvey showed that blade sweep leads to increased profile loss due to increased blade area and pressure surface velocity (Pullan \& Harvey, 2007), and that blade sweep can reduce the secondary flow loss due to changes in blade loading at the endwalls (Pullan \& Harvey, 2008). A trade-off between profile and endwall loss is thus obtained for swept blading. The improved understanding of the effect of blade sweep was used by Yoon et al. (2014) to design non-orthogonal stators (with linear and compound sweep). The flow diffusion between stator and rotor was thus reduced, leading to an increase in stage efficiency of $0.49 \%$ (over the orthogonal stator). Investigations by Wang et al. (Wang \& He, 2010) (Wang, He, \& Wells, 2010) presented a blade design optimization for multistage turbomachines based on the adjoint gradient solution approach. Significant stage efficiency gains are illustrated for several test cases. Marn et al. (2009) investigated the possibility to shorten the intermediate turbine duct length by replacing the low pressure vane row with struts. Their design achieved a significant blading weight reduction and a similar flowfield to the base design with enlarged variations in turning due to stronger secondary flows.

Since the flow in a turbine is significantly influenced by secondary flows, many studies have investigated the potential of redesigning the endwall accordingly, and thus reduce loss. Computational and experimental studies on the effect of endwall contouring have, for example, been undertaken by Harvey et al. (2000) and Brennan et al. (2003), and Hartland et al. (2000), Rose et al. (2001) and Harvey et al. (2002) 
respectively. Their studies indicate a reduction in loss and stage efficiency improvements as a result of endwall contouring. More recent numerical and experimental studies by Germain et al. (2010) and Schüpbach et al. (2010) similarly show a stage efficiency gain of $1.0 \% \pm 0.4 \%$ for non-axisymmetric endwalls, which is attributed to reduced secondary flow loss and improvements in the midspan flow. Fillets in the vane-endwall region can similarly affect the aerodynamic behaviour in the vane passage. Zess and Thole (2002) for example showed the potential to eliminate the horse-shoe vortex system and considerably reduce the passage secondary flows.

Regardless of the subject of a design study (be it vane profile, endwalls or other turbomachinery parts), it is important to validate the predictions with detailed measurements. A design study by Cranstone et al. (2014) (Part I \& II), for example, aimed to develop a low aspect ratio stator for annuli with high endwall angles. A flow separation occurred on the rear suction surface that was not predicted by the numerical simulations and had a detrimental effect on stage performance. The authors thus stress the importance to take care of employing CFD when the blade is outside the current design space. Since the integrated combustor vane concept represents a considerable alteration to the conventional nozzle guide vanes, a variety of aerothermal measurements will be used to validate the predictions.

\subsubsection{Vane heat transfer}

The complex flowfield exiting the combustor causes thermal challenges for the turbine. The temperature profile is non-uniform due to the discrete number of combustors which also results in hot streaks and can lead to thermal problems. This challenge is intensified by the continuous strive for higher turbine inlet temperatures to increase thermal efficiency as well as further thermal stress at the endwalls due to 
the flatter temperature profile of LPM combustors (due to more effective mixing). The life of a turbine blade may be halved when the metal temperature is under-predicted by a mere 28K (Han, Dutta, \& and Ekkad, 2000). A thorough understanding and prediction of the hot gas flow physics and heat transfer involved is thus critical to fulfil operational requirements and allow sufficient component life.

Many studies have investigated the effects of inlet variations on the vanes thermal performance. Nealy et al. (Nealy, Mihelc, Hylton, \& and Gladden, 1984) for example varied the Mach number, Reynold's number, turbulence intensity, and wall-to-gas temperature ratio for a highly loaded turbine first vane. It is suggested that the boundary layer transitional behaviour, freestream turbulence intensity, aerofoil surface curvature and roughness, pressure gradient, coolant injection location, flow separation and reattachment, and shock and boundary layer interactions are the basic mechanical aspects contributing to the vane's heat transfer. Abuaf et al. (1998) investigations suggest that surface roughness not only increases heat transfer coefficients but also leads to earlier transition on the suction surface of an aerofoil. Ames et al (1997) experimentally investigated the influence of combustor-simulated turbulence intensity and lengthscale on the turbine vane heat transfer. It is shown that an increase in the freestream turbulence intensity of $4 \%$ shifts the location of transition closer to the leading edge and increases the Stanton number of up to $20 \%$ on the vane. An increase in turbulence intensity does not seem to affect heat transfer levels after transition has occurred. Furthermore, large lengthscale turbulence seems to produce lower heat transfer augmentation than small lengthscale turbulence over the baseline case at similar turbulence levels. Baringer et al. (2009) showed the influence of different total temperature and pressure inlet profiles on the vane and found a reduction of HTC levels on the hub and casing in the order of $25 \%-40 \%$ for specific inlet profiles. 
To address problems of heat transfer in the endwall region, various studies have investigated the effects of leading edge modifications and endwall contouring. The papers of Shish and Lin (2003), Lethander and Thole (2004), and Han and Goldstein (2006) for instance illustrated how a modification of the leading-edge endwall region can alter the vane's aerothermal performance.

\subsubsection{Vane cooling}

Temperatures in today's gas turbines already exceed $1600^{\circ} \mathrm{C}$ and will rise even further in the continuous struggle for higher efficiencies. This temperature is far above metal alloy's melting point (Rolls-Royce, 2015), even when thermal barrier coatings are employed. Operation at these conditions is only possible through using elaborate internal and external cooling technology. A schematic overview of a turbine's first vane cooling technology can be seen in Figure 2-5. Cold air (coming straight from the compressor) feeds the first vane and is ejected through holes on the vane surface and endwall. Minimizing coolant flow is paramount for reducing loss and increasing efficiency.

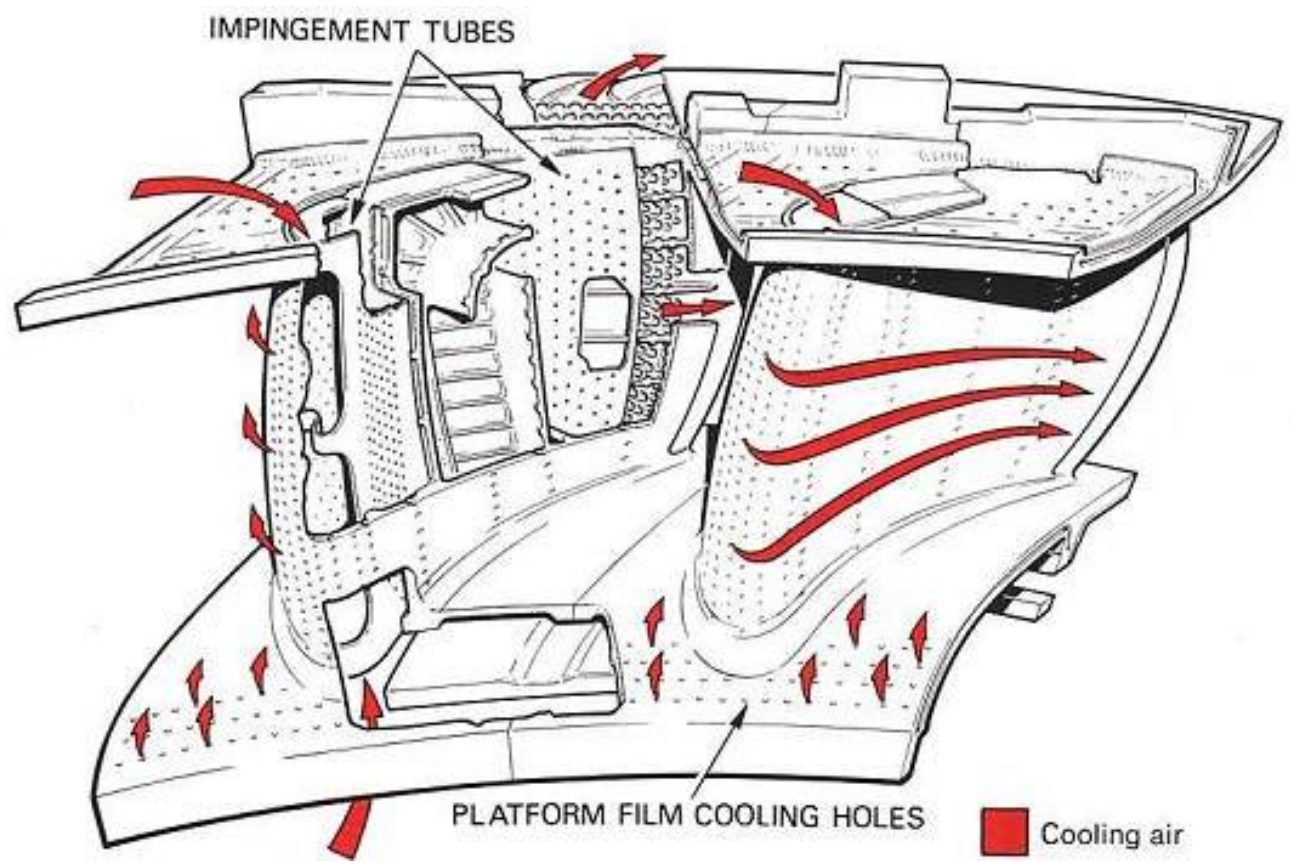

Figure 2-5: Schematic of first turbine vane cooling technology (Rolls-Royce, 2015) 
This thesis will make use of the adiabatic cooling effectiveness in order to compare different vane designs. The isothermal cooling effectiveness may be a more appropriate measure of cooling in the real engine environment, but the adiabatic cooling effectiveness provides a useful tool which is likely to obtain close ranking of cooling schemes quickly. The adiabatic cooling effectiveness is defined as:

$$
\eta_{\text {cooling }}=\frac{T_{\infty}-T_{a d}}{T_{\infty}-T_{c(o u t)}}
$$

$T_{\infty}$ is the freestream static temperature, $T_{a d}$ is the adiabatic wall temperature and $T_{c \text { (out) }}$ is the coolant temperature at the point of ejection. In compressible flow, the freestream temperature should be replaced by the recovery temperature $T_{r}$ since the deceleration of the flow towards the wall is not exactly adiabatic (Lakshminarayana, 1996). It is defined as:

$$
T_{r}=T_{\infty}+r \frac{u_{\infty}^{2}}{2 c_{p}}
$$

with the freestream velocity $u_{\infty}$, the specific heat capacity $c_{p}$ and the recovery factor $r=\sqrt[3]{P r} \approx 0.9$ for turbulent flow at typical gas temperatures. This thesis will make the simplification of $T_{0} \approx T_{r}$ which results in only a minor error for given velocities of roughly $1-2 \mathrm{~K}$. For $\mathrm{M}=1$ at ambient conditions for example, the error would be $T_{0}-T_{r} \approx(1-r) \frac{\left(330 \frac{m}{s}\right)^{2}}{2 c_{p}} \approx 5.4 \mathrm{~K}$.

The cooling effectiveness is affected by three dimensionless parameters, the density ratio (which can be derived from the other two dimensionless parameters, i.e. $\left.D R=B R^{2} / I\right)$, momentum flux ratio and blowing ratio:

$$
D R=\frac{\rho_{c}}{\rho_{\infty}} ; I=\frac{\rho_{c} u_{c}^{2}}{\rho_{\infty} u_{\infty}^{2}} ; B R=\frac{\rho_{c} u_{c}}{\rho_{\infty} u_{\infty}}
$$

In most experimental measurements $\frac{T_{C}}{T_{\infty}} \approx 1$ whereas $\frac{T_{c}}{T_{\infty}} \approx 2$ for engine conditions. In order to match the density ratio (DR), experiments thus often use a foreign gas for 
the coolant (for example $\mathrm{CO}_{2}$, which is cheap and has a density of approximately 1.67 times that of air). The momentum flux ratio (I) is important close to injection and gives an indication for the likelihood of lift-off of the coolant jet while the blowing ratio (BR) gives a measure of the local mass flux ratio and determines the mixed out behaviour of the two streams far downstream.

The prediction of film cooling performance is difficult due to the complex flowfield in today's gas turbines and the strong dependence on parameters such as the ones mentioned above. Predictions should thus if possible be complimented with experimental measurements. The two most crucial areas for first vane cooling are the leading edge and the endwalls, and a good overview is given by Bogard and Thole (2006). The leading edge area is subject to the highest heat loads due to the high heat transfer coefficient values in this region. Film cooling is achieved through rows of cooling holes called the showerhead, with detailed measurements presented by Polanka et al. (1999), Wittefeld et al. (1999), and Cutbirth and Bogard (2002). Challenges arise due to coolant separation from the surface even at low blowing ratios, and due to the varying pressure across the leading edge making the design of effective showerhead cooling difficult. Ames (1998) showed an increase in heat transfer coefficient due to film cooling, while Mehendale and Han (1992), and Reiss and Bölcs (2000) investigated various cooling hole configurations and designs.

The endwall region has become increasingly important for film cooling due to the high heat loads caused by the flatter temperature profile of modern lean premixed combustion systems. Difficulties arise due to the horse-shoe vortex system that leads to a removal of coolant from the surface. Friedrichs et al. (1996) (1997) (1999) presented detailed measurements of different cooling hole patterns. Blair (1974), and Granser and Schulenberg (1990), and Thole and Knost (2005) investigated the performance of a continuous cooling slot upstream of the vane. Their studies showed 
a significant variation of cooling effectiveness across the pitch, with the majority of cooling being convected towards the suction side. In this work, cooling will be supplied through slots. Although they have been shown to provide better cooling effectiveness compared to cooling holes (Oke, Simon, Burd, \& Vahlberg, 2000), they are generally not used in gas turbines due to mechanical constraints. They provide however a convenient test case suitable for preliminary investigations and allow a good representation of leakage flow.

\subsubsection{Influence of swirl}

Initial studies on combustor turbine interaction focused on the effects of non-uniform total temperature inlet profiles (e.g. (Hurrion, 2002) and (Haldeman, Mathison, \& Dunn, 2004)) and non-uniform total pressure profiles (e.g. (Barringer, Thole, \& Polanka, 2004)) on heat transfer, turbine life, aerodynamics, blade forcing, and efficiency. An overview of these studies is given by Povey and Qureshi (2009). More recently, investigations started focusing on combustor swirl which influences the aerothermal performance of the high-pressure turbine. Experimental and numerical studies by Qureshi et al. (Qureshi, Beretta, Chana, \& Povey, 2012) and (Qureshi, Smith, \& Povey, 2013) on the Oxford Turbine Research facility (OTRF) used a swirl simulator capable of achieving over $\pm 40^{\circ}$ of yaw and pitch at the turbine inlet. Results showed considerable spanwise variations in vane loading and a shift of the stagnation point on the leading edge (which also leads to different pressure ratios across the showerhead film cooling holes (Griffini, Insinna, Salvadori, \& Martelli, 2015)) between a uniform and a swirling flow. The considerable incidence and the convection of a vortex through the vane passage significantly affect secondary flows. Especially on the pressure surface, the strong streamline divergence poses challenges for the cooling design. Also, changes in the rotor relative inlet whirl and total pressure distribution are highlighted. The inlet swirl furthermore changes the 
Nusselt distributions on the vane surface and endwall. An increase of the Nusselt number on the rotor suction surface of $7 \%-13 \%$ was attributed to increased turbulence, while an increase of $8 \%-40 \%$ on the rotor pressure surface is attributed to increased turbulence and secondary flows. Beard et al. (2013) also showed a $1.24 \%$ mixed-out reduction in stage efficiency with swirl primarily due to altered vane loading with off-design incidence, and comparatively small changes to the rotor. All this shows significant implications of inlet swirl on the vanes aerodynamics, heat transfer and cooling design. Schmid et al. (Schmid, Krichbaum, Werschnik, \& Schiffer, 2014) used a combustor emulator together with a 1.5-stage axial turbine to show the influence of different swirl orientations and clocking positions on the turbine's aerothermal performance and also found a reduction of turbine efficiency for the swirl case - mainly due to increased turbulence and incidence. Inlet swirl also influences the hot-streak migration as shown by Khanal et al. (2013) and Rahim \& He (2015) by leading to a radial migration (depending on swirl direction and vortex-NGV clocking) of the hot fluid within the NGV passage and thus altering the aerothermal performance, as shown on simulations on Rolls-Royce's inhouse code Hydra. Experimental and numerical studies by Jacobi et al. (2017) identified and explained the formation of a secondary flow like structure around the first vane -caused by the interaction of the combustor's residual swirl core and vane potential field- with large temporal fluctuations in heat flux and potentially significant implications on film cooling.

\subsection{Combustor turbine interaction and integration}

In the past, combustor and turbine have usually been developed separately due to the complexities of each component, with simple boundary plots being exchanged at the interface. This has, however, been shown to be unsatisfactory. Salvadori et al. 
(2012) demonstrate the necessity to model the combustor in conjunction with the turbine by comparing a decoupled with a loosely-coupled steady RANS simulation. This approach is proven to be mandatory for an adequate representation of the thermal field and vane row exit temperature. Cha et al. (2012) similarly show that using simple inlet profiles for turbine development does not adequately represent the complex flow at the combustor-turbine interface and that NGVs can have a significant effect on the flow upstream of the turbine. The downstream rotor on the other hand does not significantly affect the vane's heat transfer, as shown by Dunn et al. (1985). Furthermore, it is common practice to use steady CFD as a design tool. Although steady methods may capture some basic flow features, turbomachinery flows are naturally unsteady. In fact, the evidence of instability in swirl combustion systems has been extensively reported in literature (Huang \& Yang, 2009) (Syred, 2006). Therefore, unsteady flow simulations seem to be needed to characterise the complex mechanisms occurring in LPM combustors, to properly investigate the effect of inlet non-uniformities in gas turbines, and to capture the correct physics of hot-streak migration (Takahashi \& Ni, 1990). Cha et al. (2012) furthermore highlight that modelling NGV passages with LES instead of two-equation RANS models could be beneficial for simulating the turbulence. You et al. (2008) performed an integrated simulation including fan, compressors, turbines (URANS) and combustor (LES) for a $20^{\circ}$ sector of a Pratt \& Whitney gas turbine engine and illustrated that the flow exiting the combustor features fine-scale velocity fluctuations and intermittent large-scale flow structures.

Besides efforts to understand the physics of these complex flow interactions and their effect on the HP turbine's aerothermal performance there are some attempts to alter the flow interaction of the combustor-turbine interface by physically integrating combustors and NGVs. Rosic et al. (2011), for instance, suggest minimising the axial 
distance between can combustor wall trailing edge and NGV leading edge, thus shielding the NGV. This helps to diminish the detrimental impact of the vortices shed from the gas turbine can combustor walls on the downstream NGVs' film cooling effectiveness (Mazzoni, Klostermeier, \& Rosic, 2014). The approach furthermore allows for a removal of every second NGV's showerhead, thus reducing the amount of coolant air and simplifying the NGV's internal cooling architecture. Minimizing the distance between combustor wall and nozzle guide vane also leads to a removal of the shielded vane's horse-shoe vortex system which can lead to an improvement of vane aerodynamics and endwall cooling effectiveness, as shown by Aslanidou et al. (2011).

A further design evolution, the Integrated Combustor Vane concept first introduced by Rosic et al. (2011), is presented. It suggests achieving the flow turning with vanes that are fully integrated into the combustor walls and thus eliminate the need for NGVs altogether. For very long, the focus in industry has been to control and reduce losses by applying different 3-dimensional features in blade design. The work of Denton (1999) explains the physics of three main 3-dimensional features usually applied in blade design (lean, sweep, twist). These mechanisms are however based on conventional turbine design wisdom. Since the integrated vane concept significantly alters the first vane geometry, passage structure and thus the overall flowfield, a thorough investigation of the integrated vane concept should highlight performance aspects such as aerodynamics, heat transfer, cooling and manufacturing. This is essential to draw conclusions on IV-concept's applicability, potential benefits and limitations. 


\subsection{Objectives of the Study}

This work focuses on the interface of the combustor and high-pressure turbine of power generation gas turbines with individual can combustors. Figure 2-6a shows a cut through an MHI F-type gas turbine, and the area of interest is highlighted in red. More specifically, this thesis will investigate the potential of a physical integration of the combustor and turbine. In the Integrated Combustor Vane concept, the first vanes of the high-pressure turbine (which will be called 'conventional vanes') are replaced by vanes that effectively prolong the combustor walls (which will be called 'integrated vanes'). The new integrated vanes are still separate components and thus allow the same assembly method as conventional vanes. Figure 2-6b \& Figure 2-6c illustrate the concept's suggestion for geometrical change at the combustor-turbine interface. The study firstly describes the development and aerodynamic investigation of these integrated vanes using experimental measurements on a linear high-speed cascade as well as steady RANS simulations with the in-house code TBLOCK. The key design goal was to achieve the necessary turning while reducing aerodynamic loss. The thermal performance of the integrated vane design was subsequently investigated numerically and experimentally. Annular full stage simulations confirm the feasibility and benefits of the integrated vane design for real gas turbine applications. Subsequently, the influence of an engine realistic combustor flow with swirl on the integrated vane's aerothermal performance is investigated and potential drawbacks are addressed with three-dimensional integrated vane profiling. Finally, cooling designs were developed to effectively cool the integrated vane surfaces and endwalls, and the results are compared to the conventional vane's cooling characteristics. 


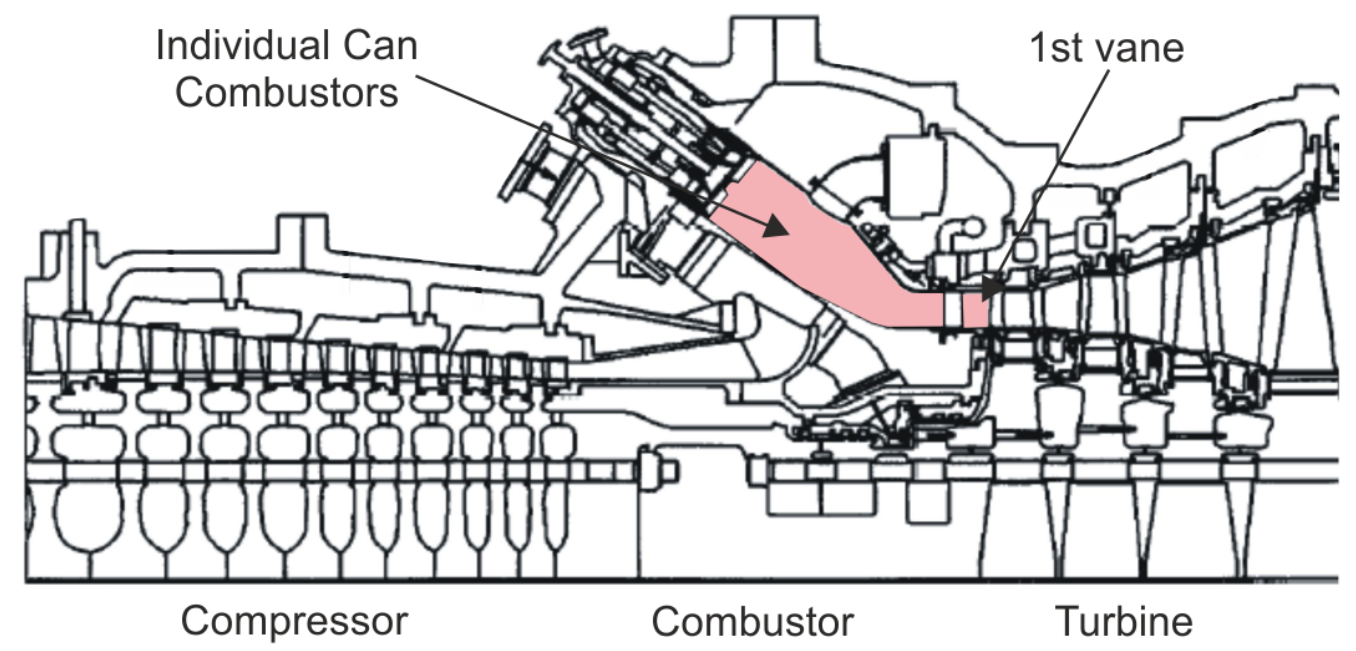

a) F Type gas turbine (Mitsubishi Heavy Industries Ltd.)

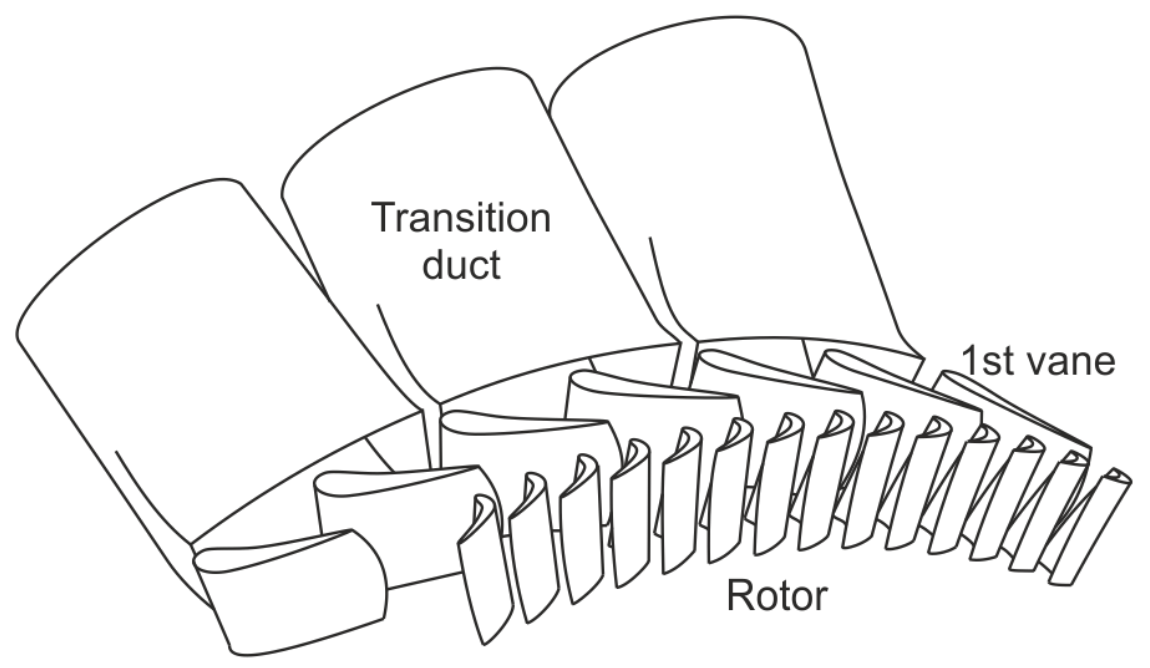

b) Conventional separated combustor and vane design

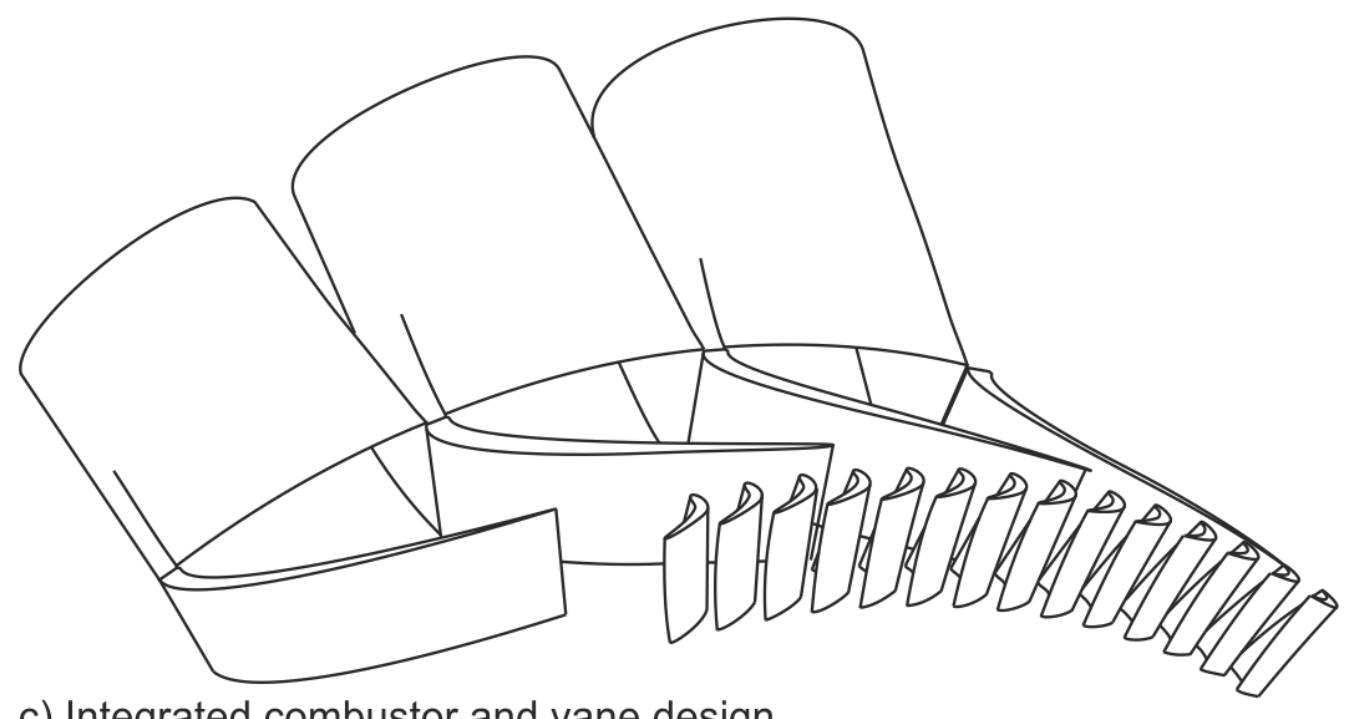

c) Integrated combustor and vane design

Figure 2-6: Cut through an MHI F-type power generation gas turbine (a), and schematic interface of combustor and high-pressure turbine for conventional (b) and integrated (c) vane design 


\section{Chapter 3: Experimental Methodology}

\subsection{Abstract}

This chapter will introduce the reader to the working principle of the high-speed combustor-turbine research facility. The working section will be presented for a conventional and integrated vane design, and the three main flow inlet conditions (comprising a no-swirl, swirl and reversed swirl case) will be described. Moreover, the measurement techniques for aerodynamic and thermal experiments are explained in detail. Finally, an overview is given on the design and functioning of the cooling system.

\subsection{Experimental facility}

The experimental facility used in this study is a linear cascade operated at scaled engine conditions (Luque, Kanjirakkad, Aslanidou, Lubbock, \& Rosic, 2015). It has been developed for the acquisition of detailed aerothermal measurements in the interface region between the combustor and turbine's first stage nozzle guide vanes. It is a blow-down type facility that is high subsonic, non-rotating, and non-reactive. The facility is scaled down to $64 \%$, and matches the Mach and Reynolds numbers and inlet turbulence intensity, thereby non-dimensionally achieving near engine conditions. Figure 3-1 shows a CAD overview and a photo of the experimental facility. The control panel in the photo operates the inlet valve and heater mesh. Air to the experimental facility is supplied from a high pressure reservoir at atmospheric temperature (2 metric tonnes at 27.6 bar) and delivered to the test section at approximately $4.88 \mathrm{~kg} / \mathrm{s}$ via a high mass flow rate regulator and a critical flow Venturi nozzle, which maintain a constant pressure of 1.6 bar $( \pm 0.5 \%)$ at the inlet of the working section. Prior to the working section, the flow is conditioned using perforated plates and a honeycomb panel to achieve uniform inlet conditions. The working gas 
is finally exhausted to a silencer at atmospheric conditions. The mainstream flow is heated by using a steel wire heater mesh, which dissipates up to $150 \mathrm{~kW}$ of $\mathrm{DC}$ power for a maximum temperature rise of $30^{\circ}$.

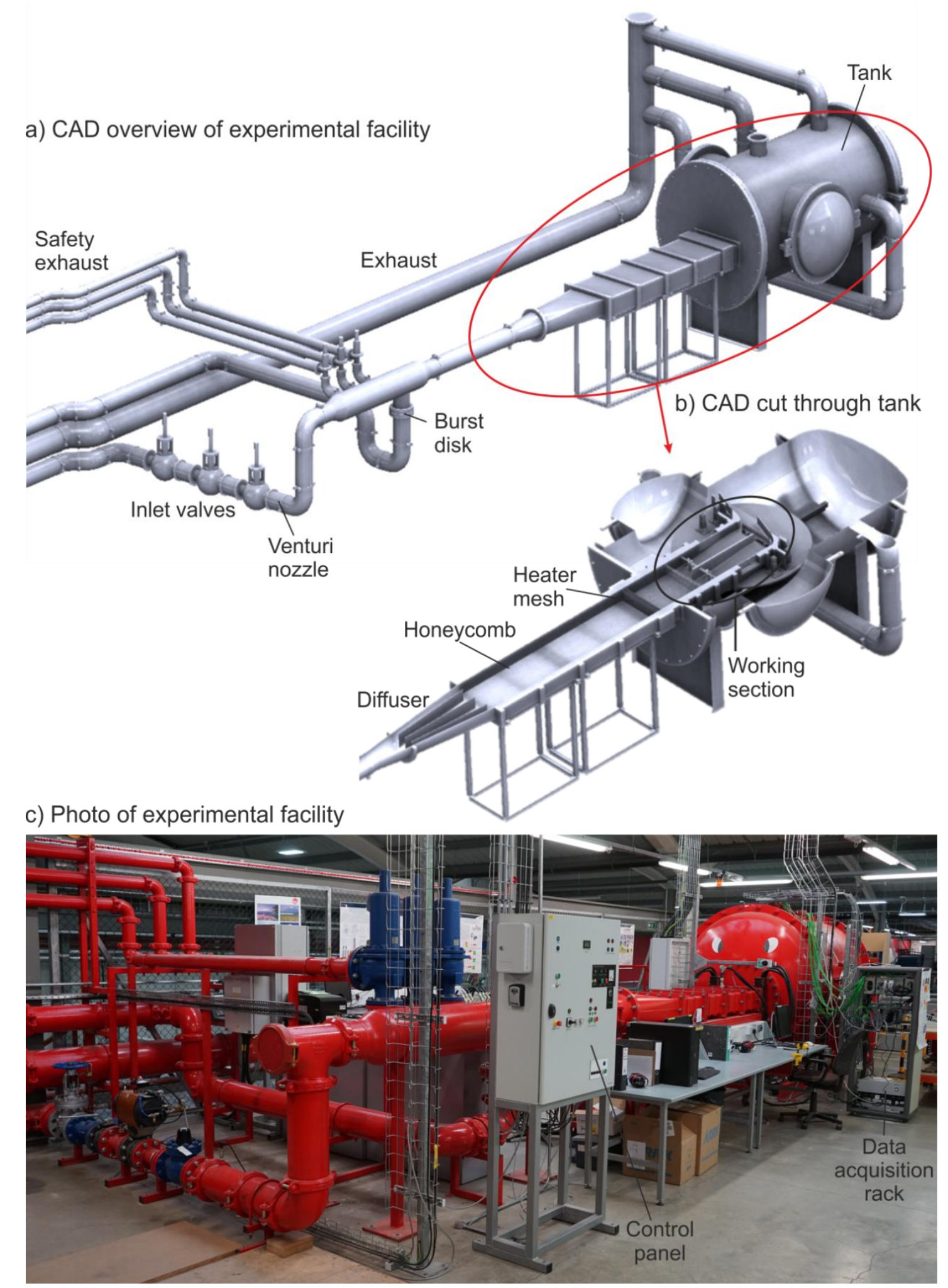

Figure 3-1: CAD overview (a)(b) and photo (c) of the high-speed combustor turbine research facility 


\subsection{Working section}

The working section is located inside of the experimental facility's tank. It has a modular design that allows for a very quick and flexible adaptation of the main components. Figure 3-2 shows the CAD geometry of the working section and a photo of the linear cascade.

a) CAD overview of linear cascade with conventional vanes

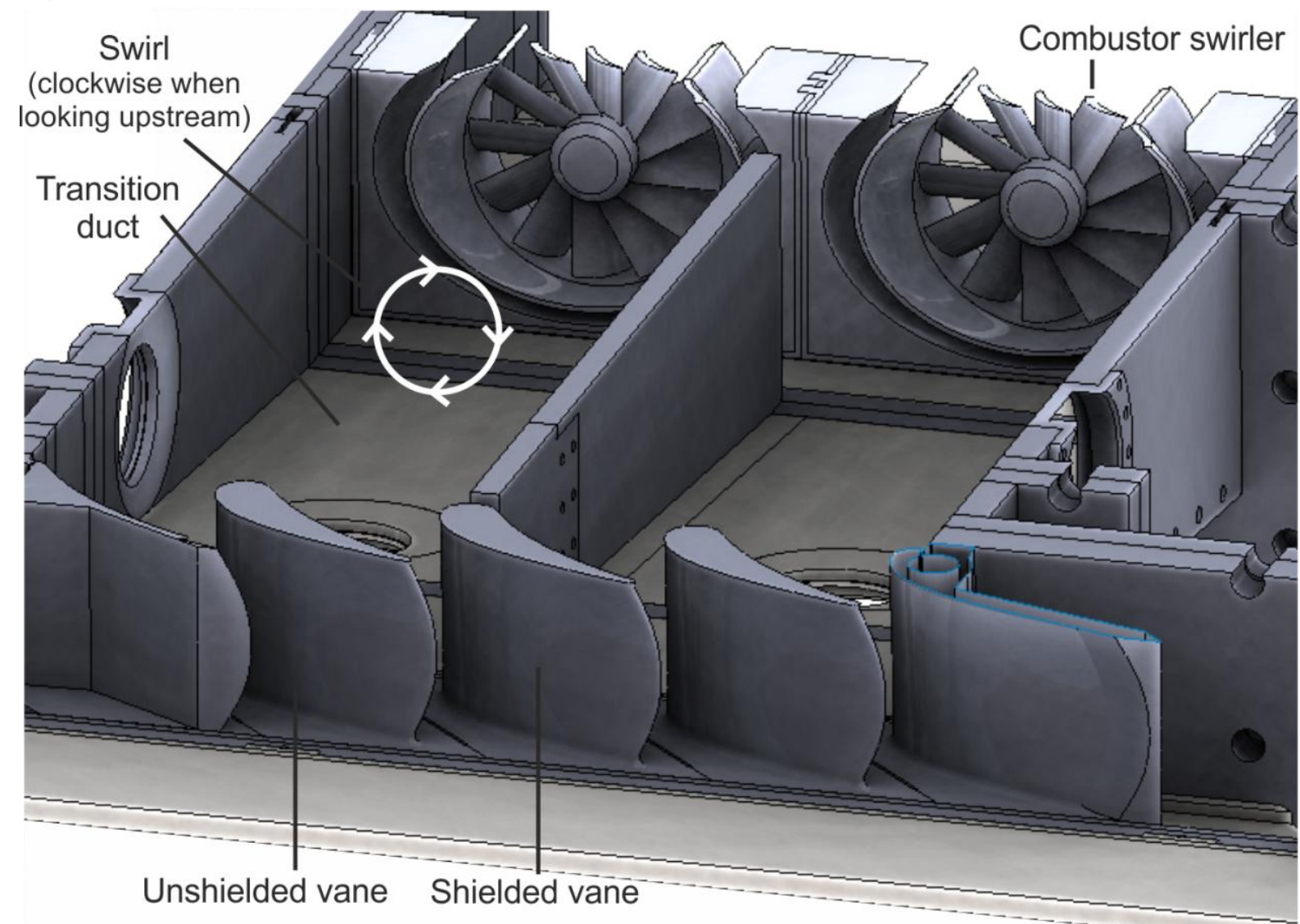

b) Photo of conventional vane linear cascade

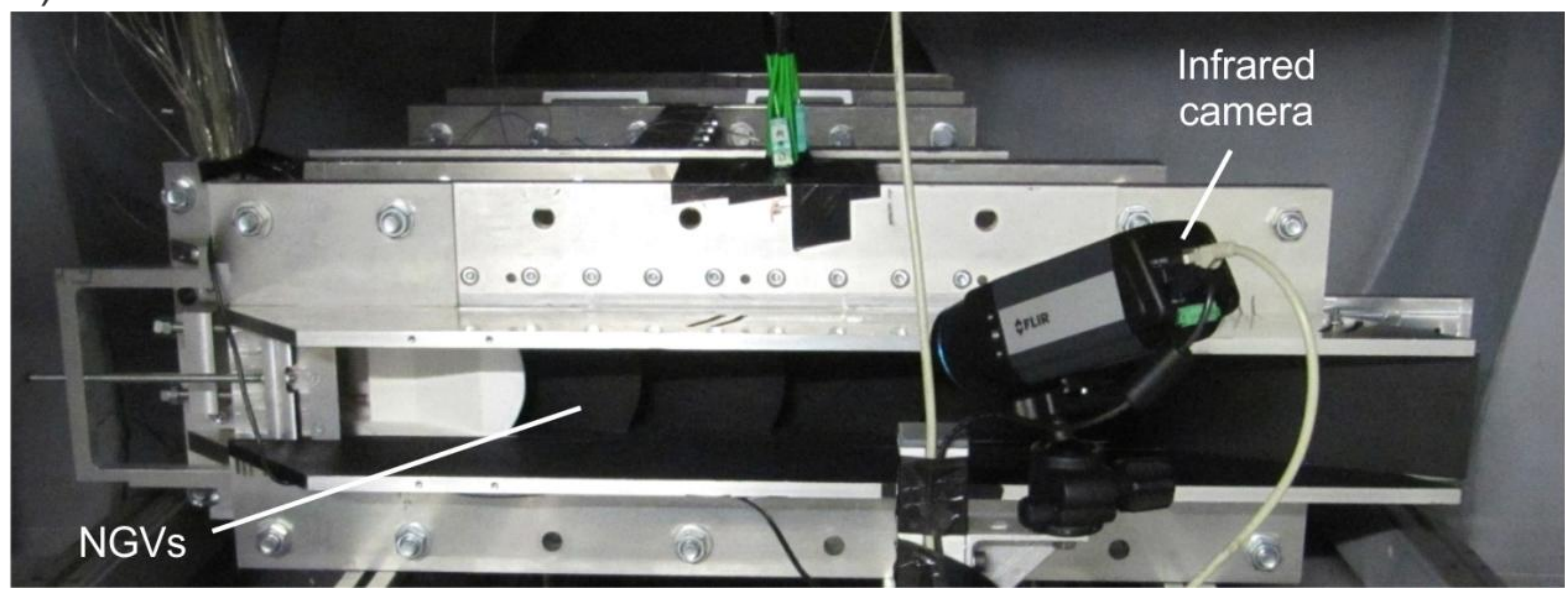

Figure 3-2: CAD (a) and photo (b) of the experimental facility's linear cascade (view from downstream), including swirlers, transition ducts and conventional vanes 
It models two can combustors including inlet cassette (in this case swirlgenerators) and transition duct, four high-pressure nozzle guide vanes, and tailboards (not shown) for flow periodicity. It can be seen that there are two conventional vanes per combustor. One of these vanes is 'shielded' by the upstream combustor wall while the other is 'unshielded' (i.e. in the centre of the duct), as indicated in Figure 3-2a. Furthermore, the CAD figure indicates the direction of the swirl (white arrows), which is defined as clockwise when looking upstream. The infrared camera in the photo is used for thermal measurements and can be installed in various locations up- and downstream of the vanes.

Figure 3-3 similarly shows the working section with the novel integrated vanes which are located downstream of the combustor walls. There is thus no vane in the centre of the passage anymore. The CAD figure also shows a window that allows acquisition of thermal measurements from upstream using the infrared camera. The photo in Figure 3-3b shows the actual integrated vane working section hardware. The working section was designed for this project to be easily attached to or detached from the combustor transition duct (sealed by an O-ring). The novel integrated vanes are simply slid into the working section. Also the mechanism for fixing and clocking the integrated vanes can be seen. The vanes are painted black (using 3M Nextel velvet coating $811-21$, primer 5523 , and hardener 5524 ) to achieve low reflectivity in the thermal measurements. 
a) CAD overview of linear cascade with integrated vanes

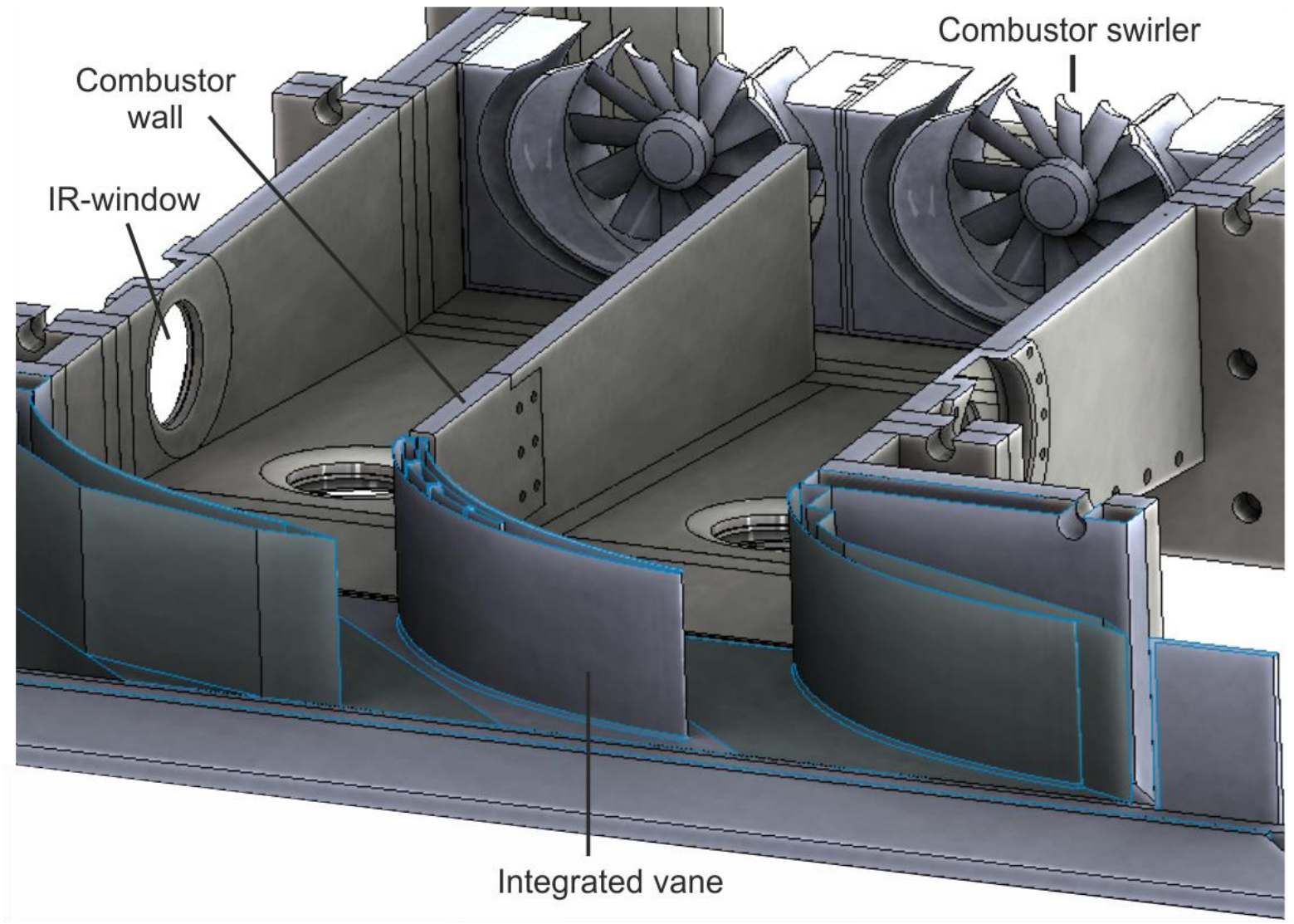

b) Photo of integrated vane working section

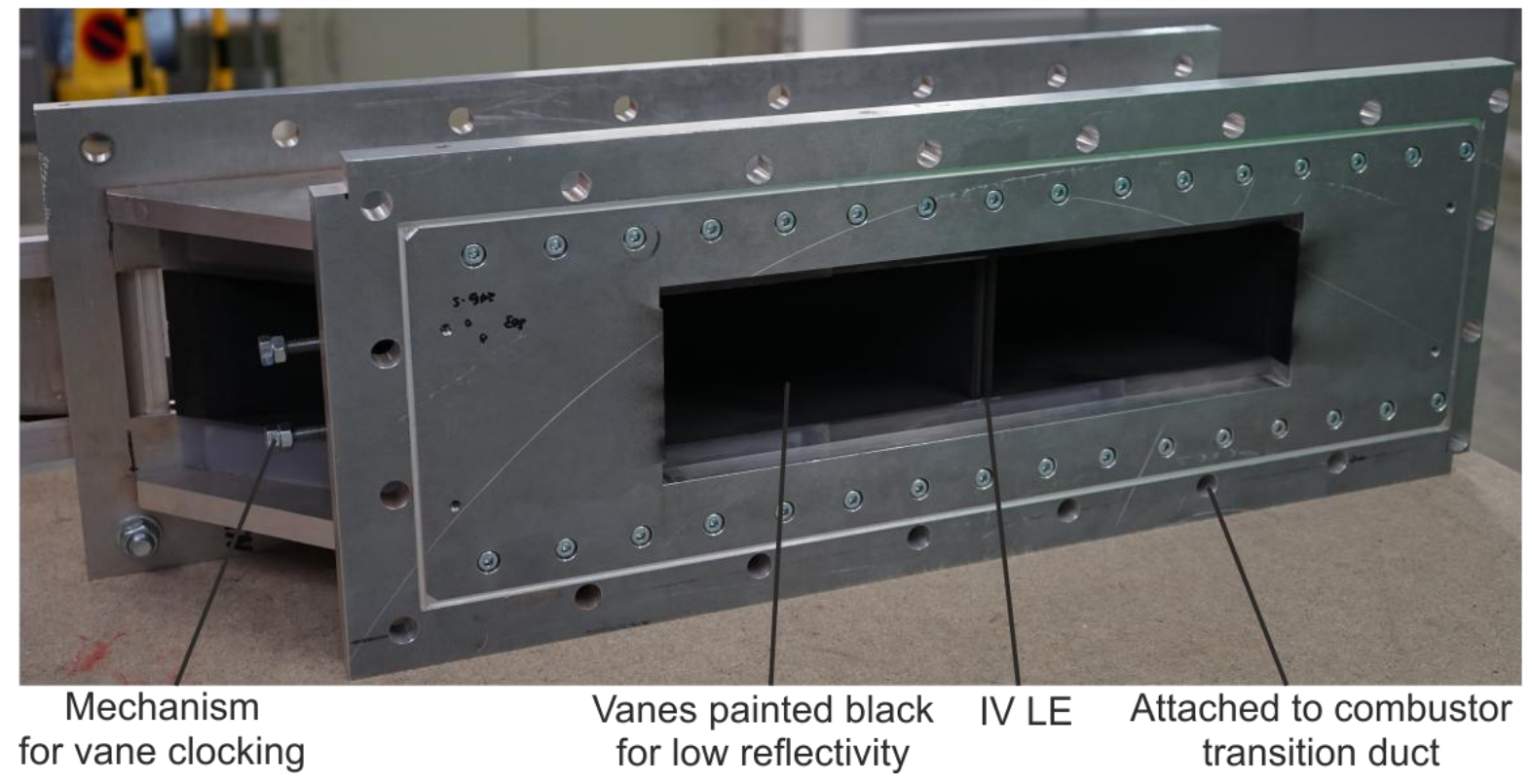

Figure 3-3: $C A D$ of the experimental facility's working section (view from downstream), including swirlers, transition ducts and integrated vanes (a) and photo of integrated vane working section (view from upstream) (b) 


\subsubsection{Flow periodicity}

The flow in the working section is well conditioned. Despite the relatively low number of components (i.e. 2 ducts and 2-4 vanes) flow periodicity is achieved by employing tailboards downstream of the vane sidewalls which are inclined by approximately $72^{\circ}$ to guide the flow. The resulting flow periodicity can be seen in the isentropic Mach number measurements (Luque, Kanjirakkad, Aslanidou, Lubbock, \& Rosic, 2015) performed at the midspan of three conventional vanes as seen in Figure 3-4. It is evident that the Mach number levels are nearly identical except for the leading edge region of vane 2 which is influenced by the upstream combustor wall.

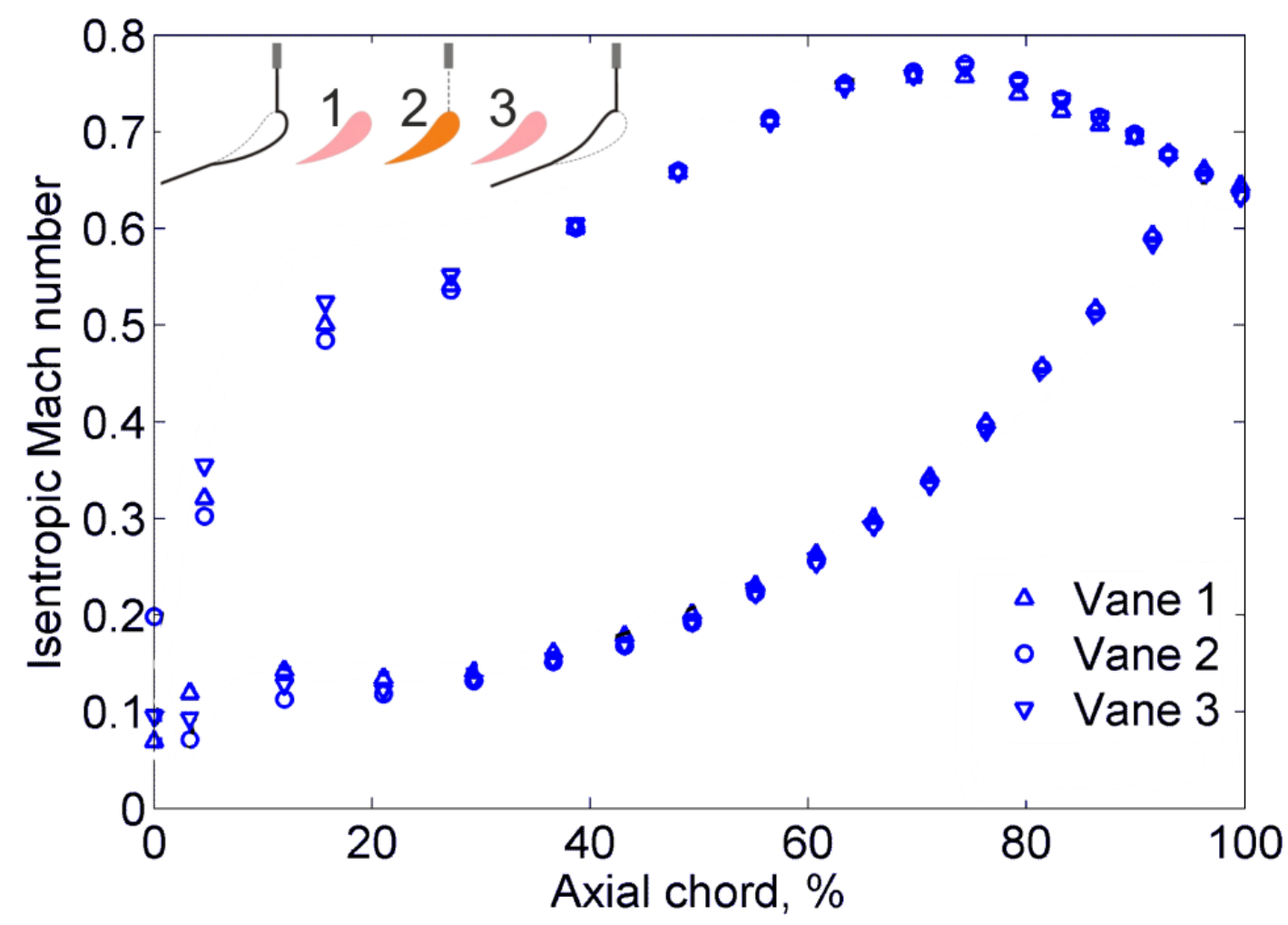

Figure 3-4: Measured isentropic Mach number around conventional vanes, adapted from (Luque, Kanjirakkad, Aslanidou, Lubbock, \& Rosic, 2015)

\subsubsection{Inlet conditions}

The facility is highly modular and allows quick adaptations of the flow conditions by exchanging cassettes upstream of the transition ducts. Three different cassettes 
allow testing a swirl scenario, reversed swirl scenario and a no-swirl scenario which represent engine-realistic combustor flow conditions. The no-swirl scenario constitutes an upstream turbulence grid (with $45 \mathrm{~mm}$ diameter holes and a thickness of $5 \mathrm{~mm}$ ) that results in approximately $12 \%$ turbulence intensity (estimated through empirical correlations by Roach (1987)) and the swirlers result in an averaged swirl angle $\tan ^{-1}\left(\frac{v_{t}}{v_{x}}\right)$ of $\pm 8^{0}$ at the inlet of the vanes (more detailed information can be found in (Jacobi, 2013)). The cassettes for the turbulence grid and reversed swirler can be seen in Figure 3-5.

a) Turbulence grid representative of no-swirl case

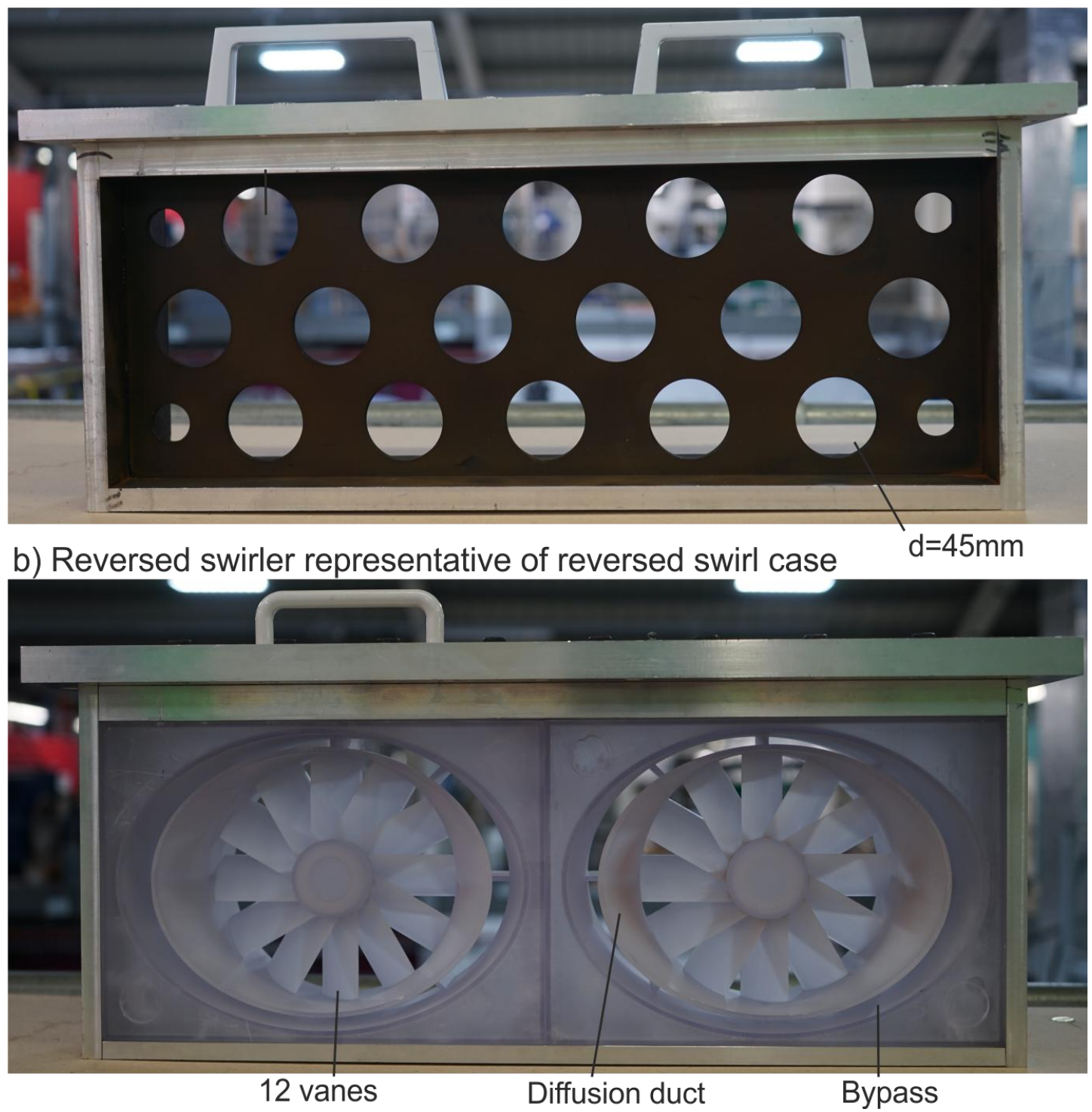

Figure 3-5: Photo of inlet cassette showing turbulence grid (a) and reversed swirler (b) 
The swirl strength is slightly higher than in industrial practice (approximately $6^{\circ}$ for a swirler similar to the technical drawing in Figure 2-2) to illustrate the effect of the swirl on the vanes aerothermal performance more clearly. The swirl-generators have a generic geometry with one pilot swirler, a diffusion duct and a bypass which result in flow features commonly found in combustors, such as a recirculation zone and a precessing vortex core. The swirler with reversed swirl direction is obtained by mirroring the swirler geometry on a vertical plane.

\subsection{Measurement capabilities}

\subsubsection{Aerodynamics}

Aerodynamic measurements include up- and downstream (of the vane) fully resolved automated area traverse measurements with a 5-hole probe and a pitot probe, and static pressure measurements on the vane surfaces using a Digital Sensor Array (DSA 3128, Scanivalve Corp.) box with 16 pressure transducers and a measurement accuracy of $0.05 \%$. The 5 -hole probe was designed by V. Kanjirakkad and manufactured using laser metal sintering. It features a $2.05 \mathrm{~mm}$ head diameter and $0.3 \mathrm{~mm}$ hole diameter. A calibration of the 5 -hole probe was performed on an experimental facility consisting of a fan that ejects air through a nozzle at a constant velocity and a 2 -axis rotating table that mounts the 5 -hole probe. The obtained calibration map is shown in Figure 3-6a. It can be seen that the calibration map is very symmetric and finely resolves each $1.5^{\circ}$ increment. The probe is thus able to accurately predict flow angles of pitch and yaw of up to $\pm 30^{\circ}$ based on the pressures obtained in the five holes. This is done by calculating the yaw and pitch coefficients:

$$
c_{\text {yaw }}=\frac{p_{2}-p_{4}}{p_{5}-\frac{1}{4} \Sigma_{i=1}^{4} p_{i}} ; c_{p i t c h}=\frac{p_{1}-p_{3}}{p_{5}-\frac{1}{4} \Sigma_{i=1}^{4} p_{i}}
$$

and subsequently looking up the corresponding flow angles in the probe calibration. 

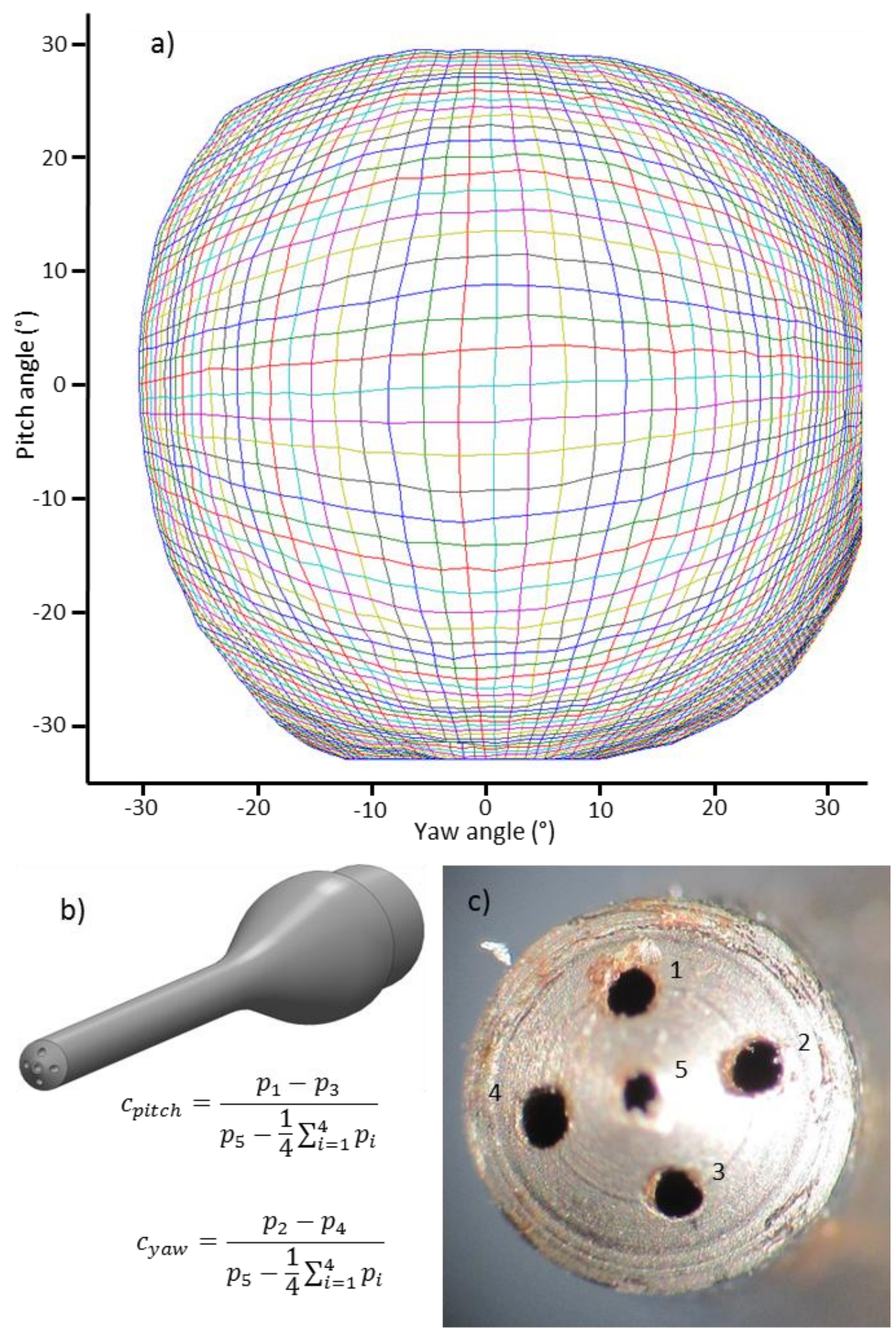

Figure 3-6: Calibration map (a), CAD geometry (b) and photo (c) of 5-hole probe 
The estimated uncertainty to within $95 \%$ confidence is $\pm 0.35^{\circ}$ (Luque, Kanjirakkad, Aslanidou, Lubbock, \& Rosic, 2015). Figure 3-6b and Figure 3-6c also show the CAD geometry of the probe and a photo (through a microscope) of the probe head. It can be seen that the outer holes are well resolved by the manufacturing process while the central hole did not quite build to the desired dimensions.

The 5-hole probe is then mounted to a fully automated 3-axis traverse mechanism that allows to measure roughly one location per second. Thus, multiple runs are necessary to resolve the entire measurement areas. The aerodynamic traverse system was used for measurements up- and downstream of the conventional and integrated vanes, and a photo of the setup is shown in Figure 3-7. The probe is installed at an angle of $70^{\circ}$ for measurements downstream of the vanes (due to the high flow angles in this area). A Labview script (written by W. Ramm) is used to define the probe's measurement locations. These are tailored to each experiment in order to finely resolve the most relevant areas (e.g. more points in vane wake than downstream of passage). 

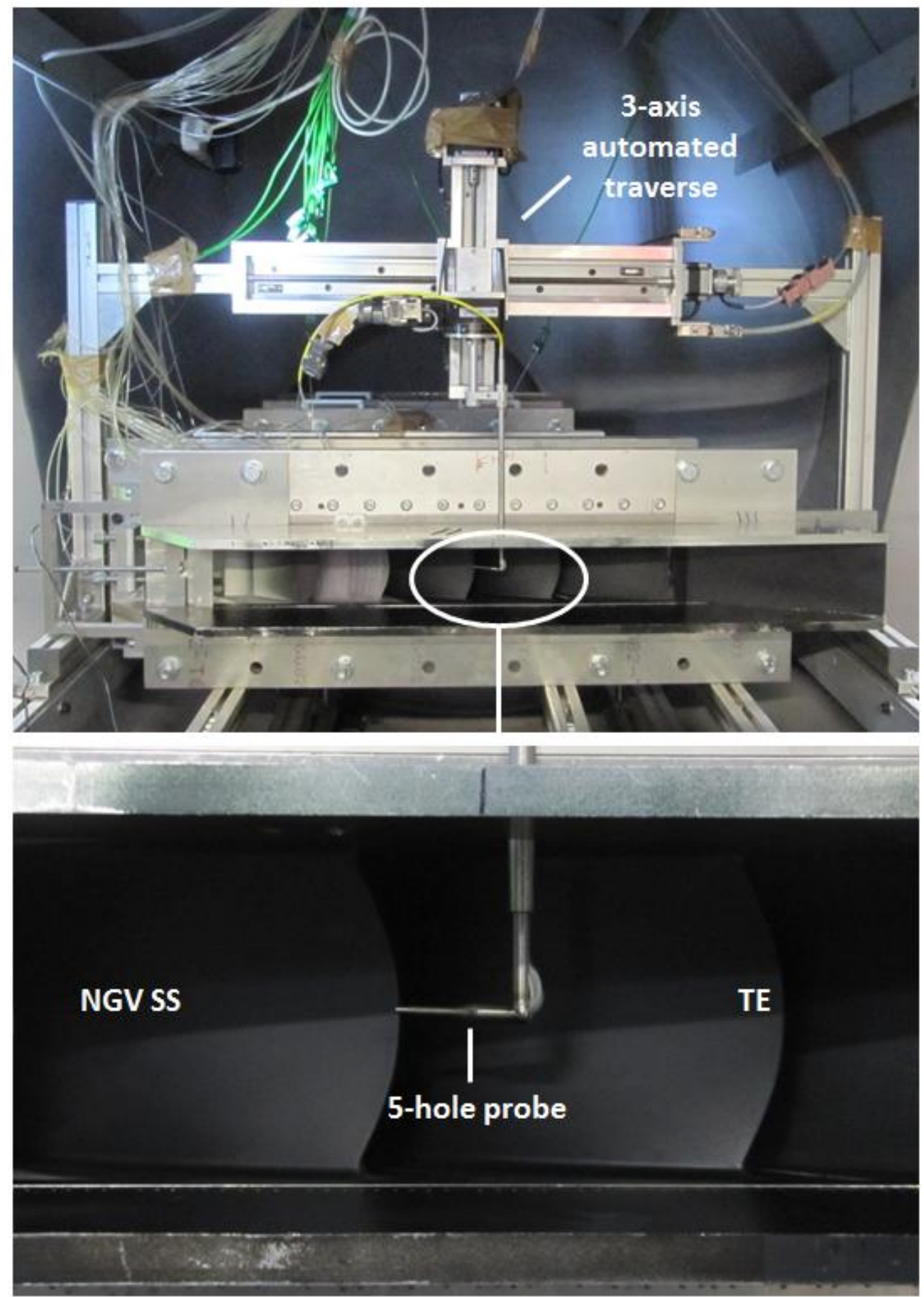

Figure 3-7: Photo of the experimental facility with automated 3-axis traverse mechanism and 5-hole probe

In order to obtain a better understanding of the flow within the passage, the integrated vane was equipped with pressure tappings. Figure 3-8 shows a photo of this integrated vane and gives a schematic overview of the position, geometry and number of the pressure tappings along the chord of the integrated vane's midspan. 
a) Photo of integrated vane with pressure tappings

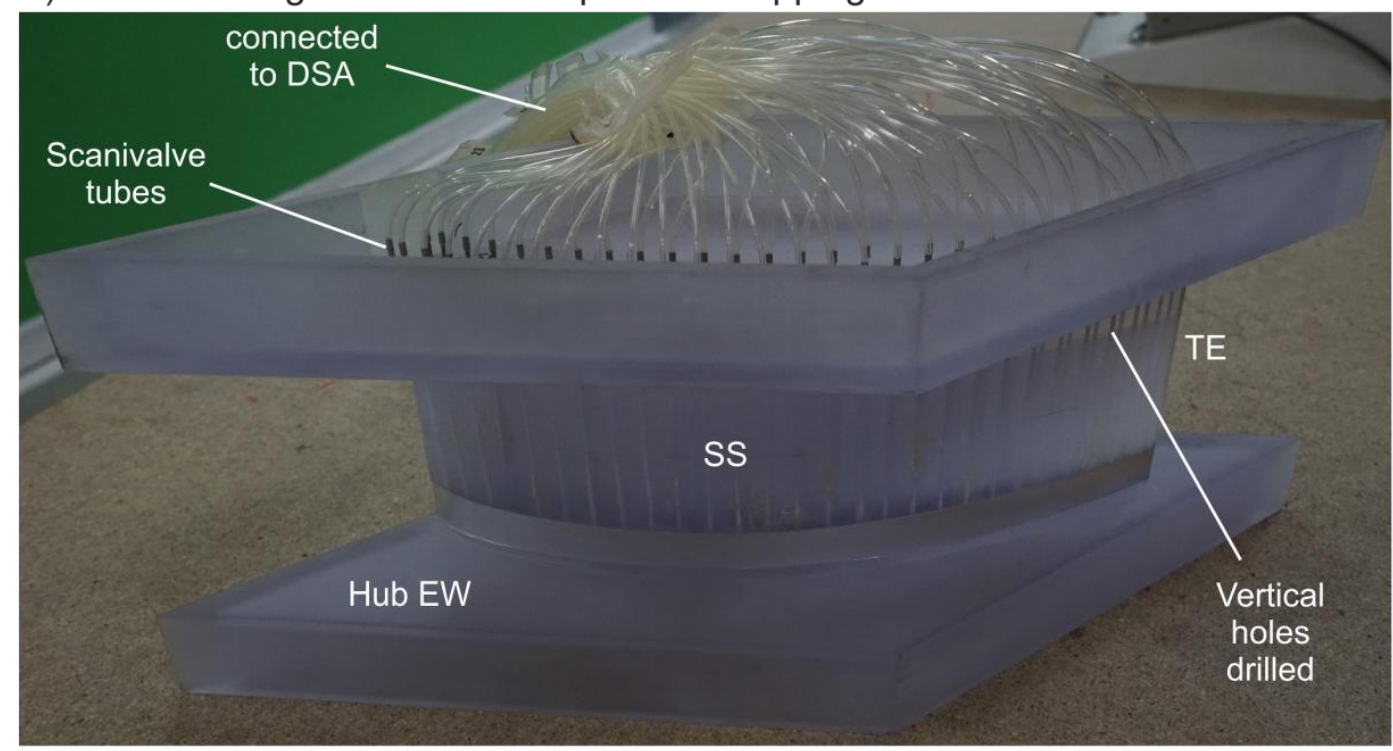

b) Schematic of pressure tappings' location \& geometry

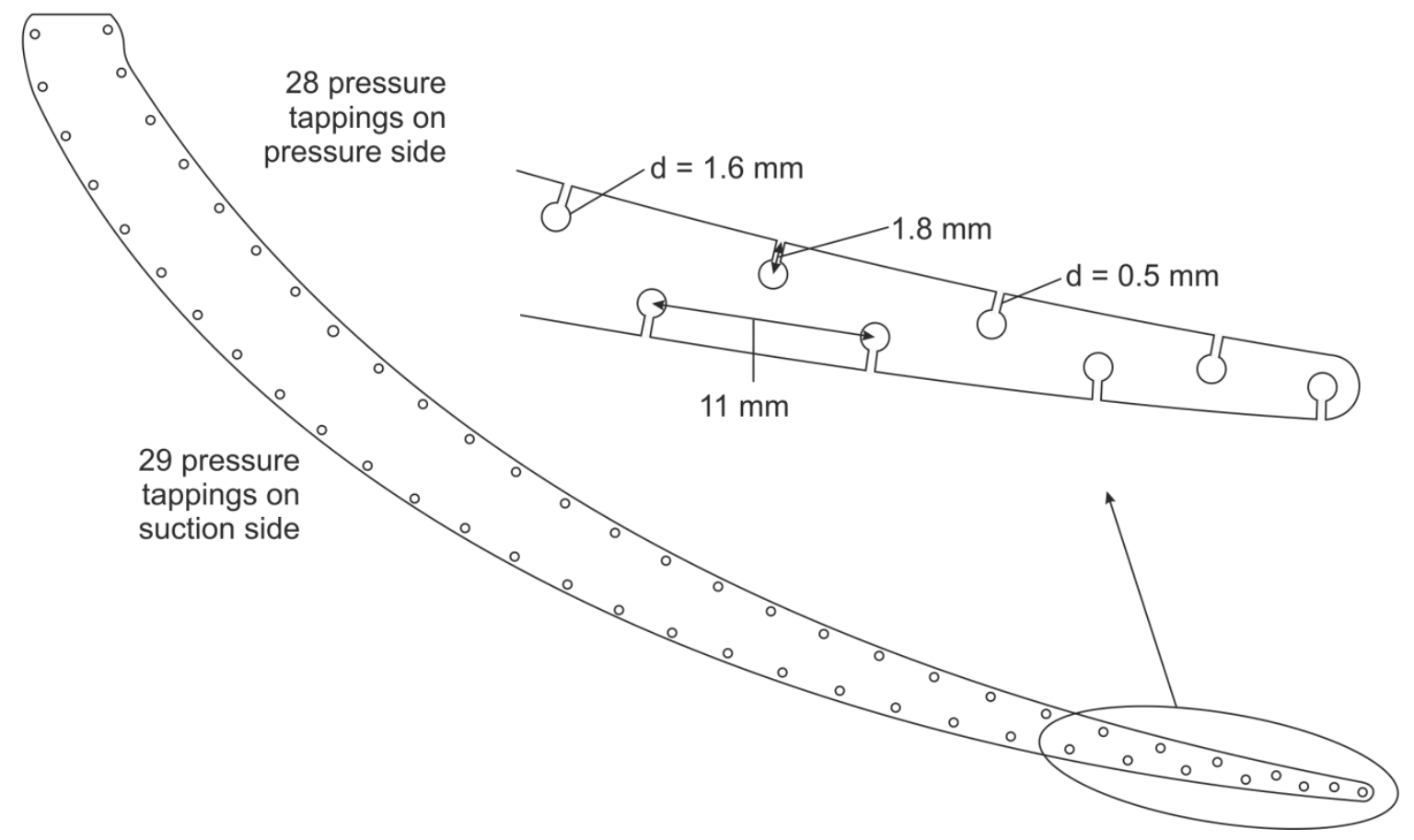

Figure 3-8: Photo of integrated vane with pressure tappings (a) and integrated vane midspan section specifying number, position and geometry of pressure tappings (b)

A vertical hole diameter of $1.6 \mathrm{~mm}$ was chosen to simplify the instrumentation using $1.6 \mathrm{~mm}$ Scanivalve tubing, and to reduce velocity and thus losses inside of the holes. The horizontal holes (connecting the vertical holes with the integrated vane outer wall) feature a diameter of $0.5 \mathrm{~mm}$ and are orientated perpendicular to the outer wall in order to measure the static pressure. Since the manufacturing process was unable 
to build such small holes, the vertical and horizontal holes were drilled in the workshop ex post. In total, the integrated vane features 57 pressure tappings, 29 on the vane's suction and 28 on the vane's pressure side.

\subsubsection{Heat transfer}

The thermal measurement method used in this study is based on infrared camera measurements. Vane heat transfer coefficient distributions are obtained by comparing the transient response of the vanes to a step-rise in incident heat flux (recorded with the infrared camera) with the analytical curve by using the impulseresponse method (Oldfield, 2008).

\section{Heater mesh}

The step rise in mainstream temperature is achieved by applying a steel wire heater mesh with $150 \mathrm{~kW}$ of $\mathrm{DC}$ power. The temperature rise can be varied between $0^{\circ} \mathrm{C}$ $25^{\circ} \mathrm{C}$ by setting the voltage at the power supply unit to between $0-110 \mathrm{~V}$. The design of the mesh is based on Gillespie's doctoral thesis (1996) and was employed in a variety of transient heat transfer experiments in the Osney Thermo-Fluids Laboratory. A picture of the heater mesh used in this work (which was designed by S. Luque) is shown in Figure 3-9. The steel wire mesh is supported around the perimeter by a Tufnol insulating frame and soldered to bus bars. To minimize the thermal stress of the mesh and prolong its lifetime (failure is usually caused by partial or complete burning of the mesh), a serial setup of two meshes was used. The wire diameter was set to $280 \mu \mathrm{m}$ (larger than in previous attempts) since this reduces the resistance of the wire (which is inversely proportional to the wire diameter). 


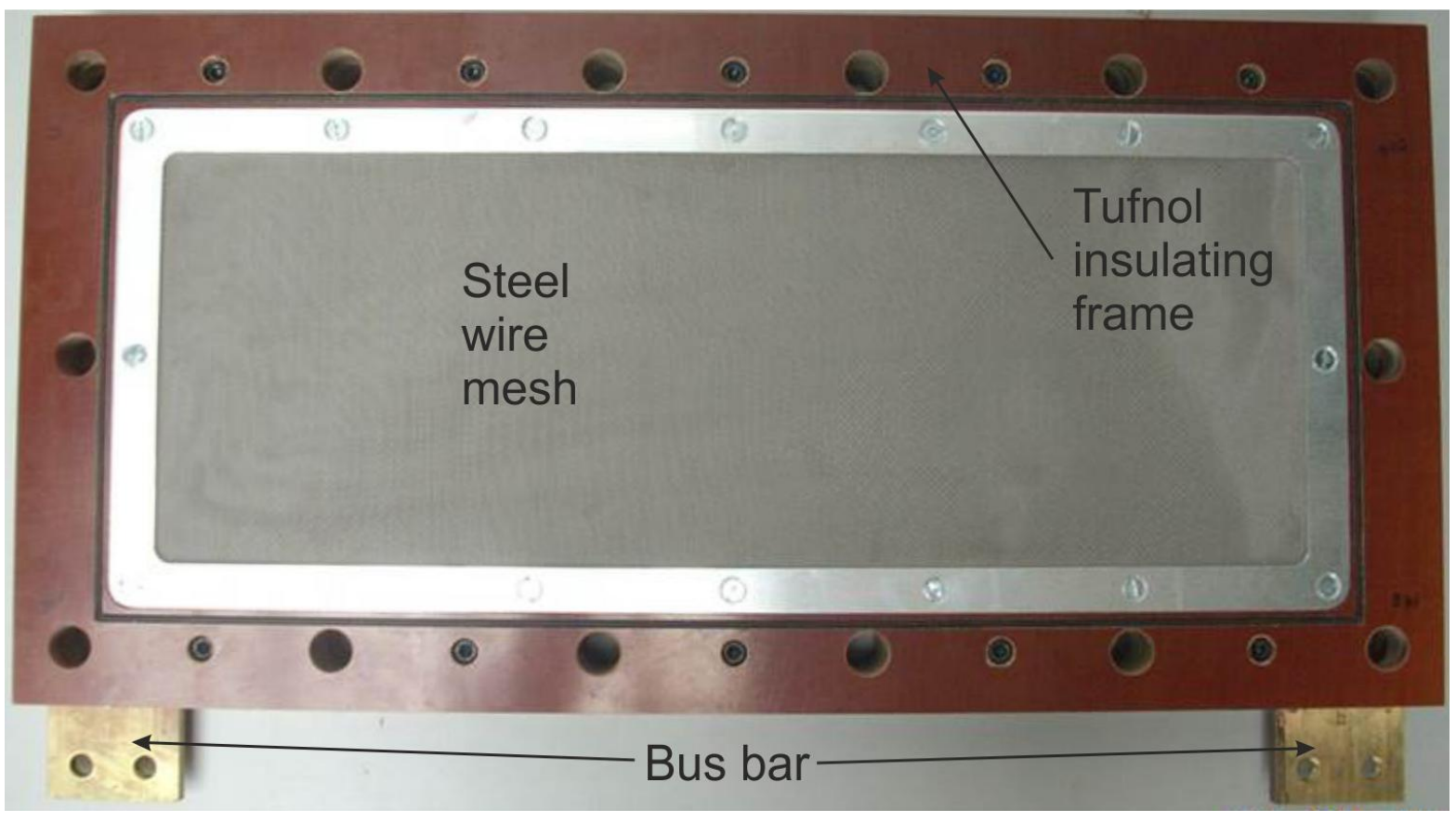

Figure 3-9: Steel wire heater mesh of the experimental facility used for heat transfer measurements

Figure 3-10 shows a typical temperature trace obtained during an experimental run.

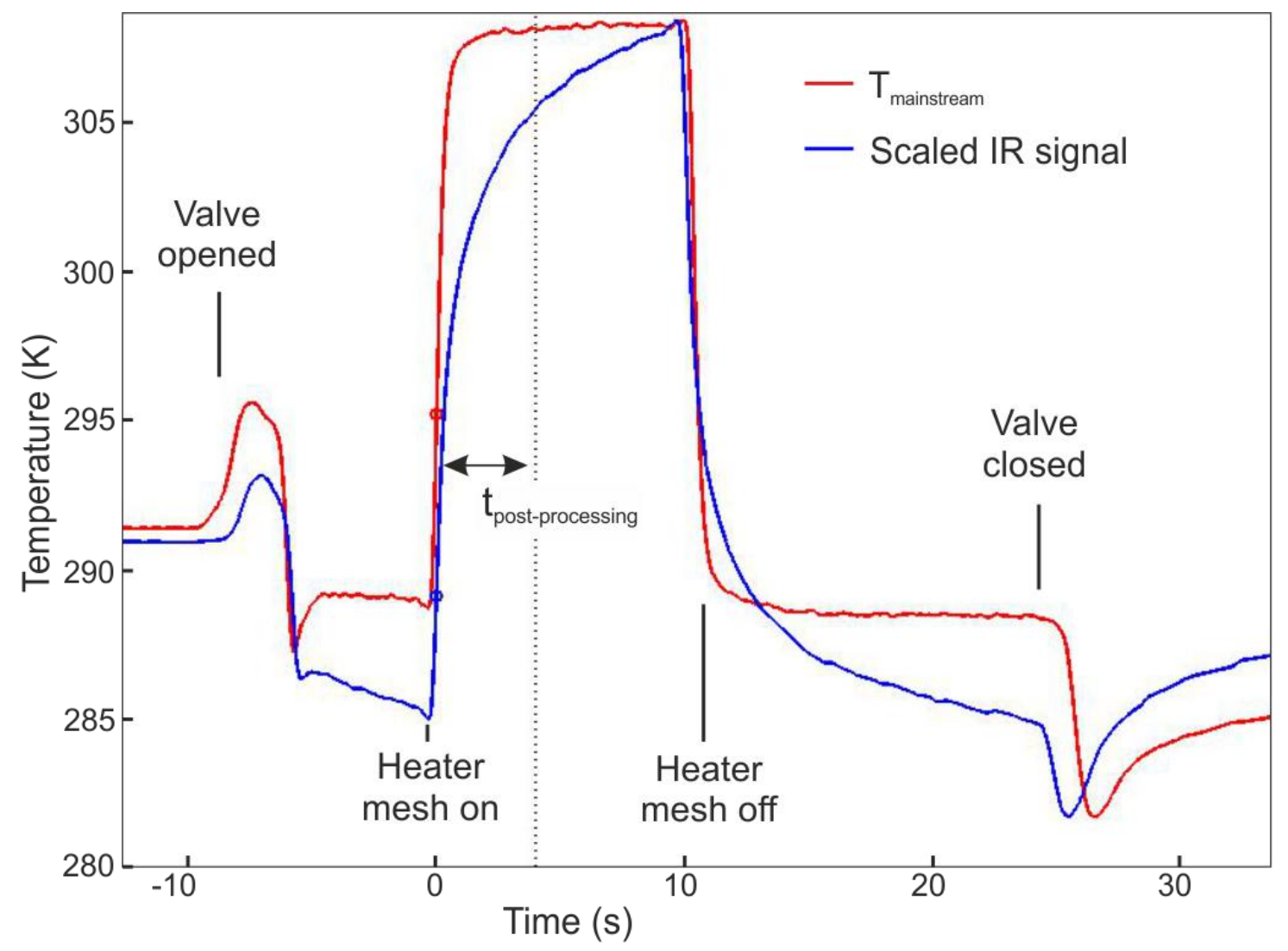

Figure 3-10: Typical temperature trace of experimental run with infrared camera 
The mainstream temperature is measured using a thermocouple in the combustor transition duct, while the wall surface temperature is measured by the infrared camera. The step rise in mainstream temperature (at $\mathrm{t}=0 \mathrm{~s}$ ), with an approximate time constant of $0.22 \mathrm{~s}$, can be seen when the heater mesh is turned on. This results in a heat flux at the wall causing the wall surface temperature to increase over time which is dependent on the material properties of the substrate.

\section{Infrared camera}

The high resolution infrared camera used for thermal measurements is the FLIR a655sc which can acquire 16-bit greyscale images at a resolution of $640 \cdot 480$ pixels and a frame rate of $50 \mathrm{~Hz}$. It uses a $25^{\circ}$ lens and has a spectral range of 7.5-13 $\mu \mathrm{m}$. Six different camera positions will be presented in this work, including two views from downstream and four views from upstream through a zinc selenide infrared window (which offers low infrared absorptivity, thus high transmission).

\section{Impulse response method}

The infrared camera's temperature measurements are processed with the transient infrared camera thermography technique to obtain heat transfer coefficient and adiabatic wall temperature distributions. To do this, Oldfield's impulse response method is used: the temperature response of the surface may be considered the convolution of the temporal impulse response function and the magnitude of the heat flux impulse at any point in time. The impulse response function can be found by discretising any known pair of heat flux inputs and wall temperature responses as a series of temporal impulses. Using the discrete Fourier transform and their inverse the transfer function from wall temperature to heat flux can be formed. The benefit of the method is that the prior wall temperature history during the period of non-constant heat transfer coefficient can be accounted for without needing to know the heat transfer coefficient transient history. 
The impulse response method is based on Fourier's law of heat conduction on a onedimensional, semi-infinite solid body:

$$
k \frac{\partial^{2} T}{\partial y^{2}}=\rho c_{p} \frac{\partial T}{\partial t} \text { with } T=T(y, t)
$$

with the thermal conductivity $k$, the density $\rho$, and the specific heat capacity $c_{p}$. For the experiments conducted in this thesis, the boundary conditions used to solve this equation are the initial temperature $T(y, t=0)=T_{i}$, a semi-infinite boundary $T(y=\infty, t)=T_{i}$, and the convective heat transfer on the wall surface $-k\left(\frac{\partial T}{\partial y}\right)_{y=0}=$ $h\left(T_{a d}-T_{w}\right)$. The analytical solution to this, described in the literature, is:

$$
\frac{T_{W}(t)-T_{i}}{T_{a d}-T_{i}}=1-e^{-\frac{h^{2} t}{\rho c_{p} k}} \operatorname{erfc} c^{\frac{h^{2} \sqrt{t}}{\sqrt{\rho c_{p} k}}}
$$

with the surface heat transfer coefficient $h$, the substrate's thermal properties (in this case the resin used for stereolithography SL7870) and the error function erfc (Weisstein). Employing the impulse response method for each of the infrared camera's pixels results in a pair of variables for each time step, namely the heat flux $\dot{q}$ and the wall surface temperature $T_{W}$. Figure 3-11 shows an exemplary $\dot{q}-T_{W}$ plot.

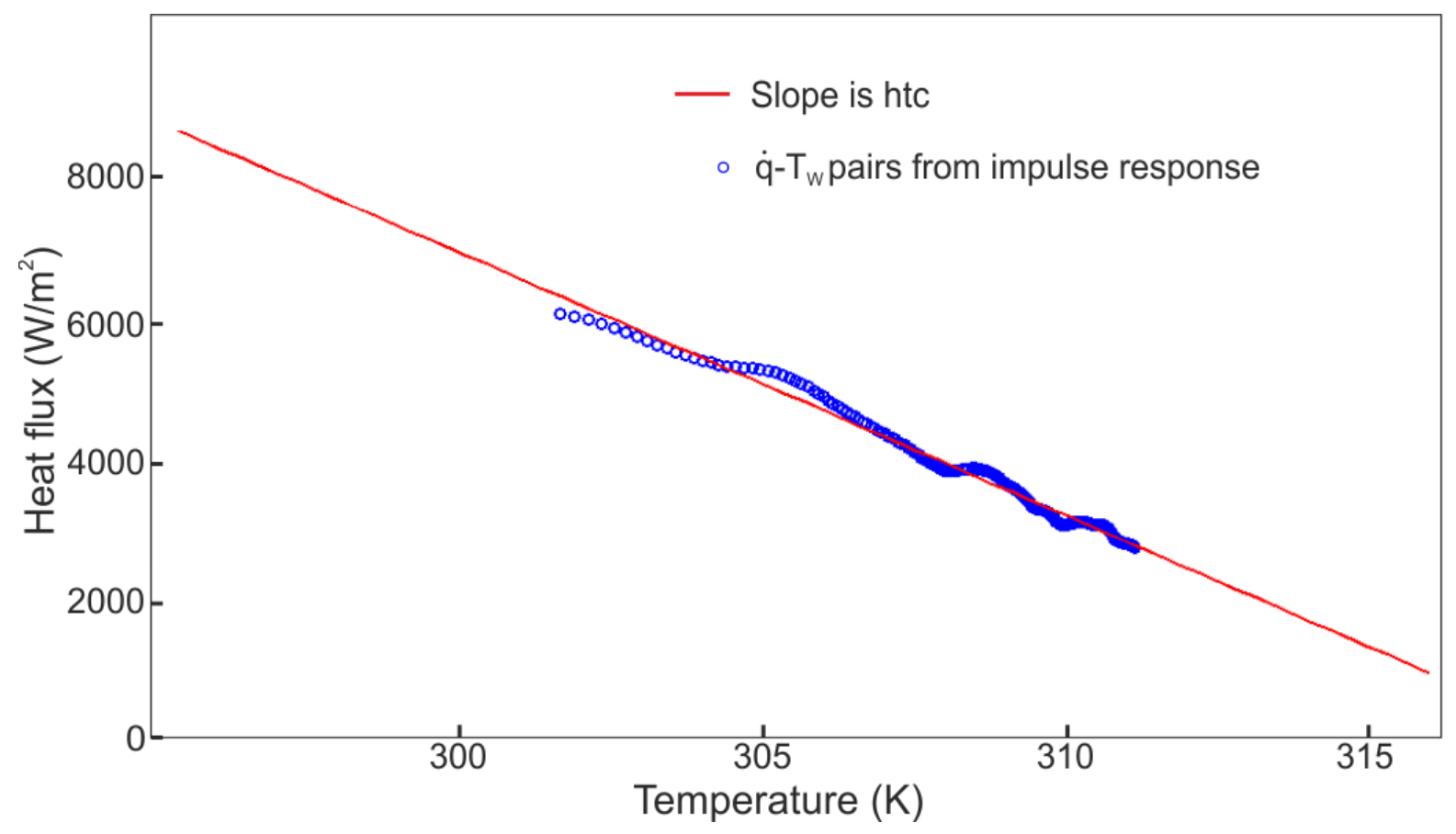

Figure 3-11: Graph of heat flux vs. temperature of the impulse response method 
The heat transfer coefficient is the slope of the linear fit and $T_{a d}$ is the $x$-axis value at $\dot{q}=0$. The Matlab routine that performs these steps was developed by Salvador Luque and is used in this thesis.

\section{Repeatability and accuracy}

The dotted line in Figure 3-10 indicates the time span that is used for postprocessing. A study was undertaken to investigate the repeatability of thermal measurements and it was found that a post-processing time of 3-4 seconds results in highly repeatable measurements independent of the day and the time between measurements. Reducing the post-processing time leads to a lower resolution of the heat transfer distributions, while increased post-processing time results in larger deviations in heat transfer coefficient levels. $A \pm 4.87 \% \mathrm{HTC}$ accuracy is achieved for an idealized heat transfer process when taking into account the uncertainty of the infrared camera measurements, material properties and the $\dot{q}-T_{W}$ least-squares fit (Luque, Kanjirakkad, Aslanidou, Lubbock, \& Rosic, 2015).

\subsubsection{Summary of operating conditions}

Table 3-1 summarizes the operating conditions in the experimental facility. A vane pressure ratio of $\mathrm{p}_{2} / \mathrm{p}_{01}=0.75$ results in an average exit Mach number of 0.65 (peak Mach number on the suction side is 0.8) with the use of the one-dimensional compressible equations for isentropic flow. This represents an engine-realistic highvelocity flow condition. A Reynold's number of $1.6 \cdot 10^{6}$ (based on axial chord at midspan) is obtained. The inlet's total temperature is approximately $320 \mathrm{~K}$ and the facility has been designed so that coolant flow is supplied by a set of vortex tubes.

\begin{tabular}{|l|l|}
\hline Condition & Value \\
\hline NGV inlet total pressure & $\mathrm{p}_{01}=1.6 \mathrm{bar}$ \\
\hline NGV outlet static pressure & $\mathrm{p}_{2}=1.2 \mathrm{bar}$ \\
\hline
\end{tabular}




\begin{tabular}{|l|l|}
\hline NGV exit Mach number & $\mathrm{M}_{2}=0.65$ \\
\hline NGV pressure ratio & $\mathrm{p}_{2} / \mathrm{p}_{01}=0.75$ \\
\hline Inlet total temperature & $\mathrm{T}_{01}=320 \mathrm{~K}$ \\
\hline Axial chord of CV \& IV & $\mathrm{C}_{\mathrm{xCV}}=90 \mathrm{~mm} \& \mathrm{c}_{\mathrm{xIV}}=160 \mathrm{~mm}$ \\
\hline Mass flow rate & $\dot{m}=4.88 \mathrm{~kg} / \mathrm{s}$ \\
\hline NGV exit Re number (based on $\mathrm{c}_{\mathrm{xCV}}$ ) & $\mathrm{Re}_{\mathrm{x}}=1.6 \cdot 10^{6}$ \\
\hline Turbulence intensity \& lengthscale & $\mathrm{T}_{\mathrm{i}}=12 \% \& \mathrm{~T}_{\mathrm{I}}=40 \mathrm{~mm}$ \\
\hline
\end{tabular}

Table 3-1: Experimental conditions of the Oxford combustor turbine research facility

\subsection{Cooling system}

In order to extend the capabilities of the experimental facility and make testing as realistic as possible, a cooling system was devised. It was developed by Salvador Luque, Roderick Lubbock and Ioanna Aslanidou, and commissioned with the help of Simon Jacobi. A constant stream of cold air (below ambient temperature) is supplied by a set of vortex tubes and finally feed the vanes and endwalls. A schematic of such a vortex tube is shown in Figure 3-12.

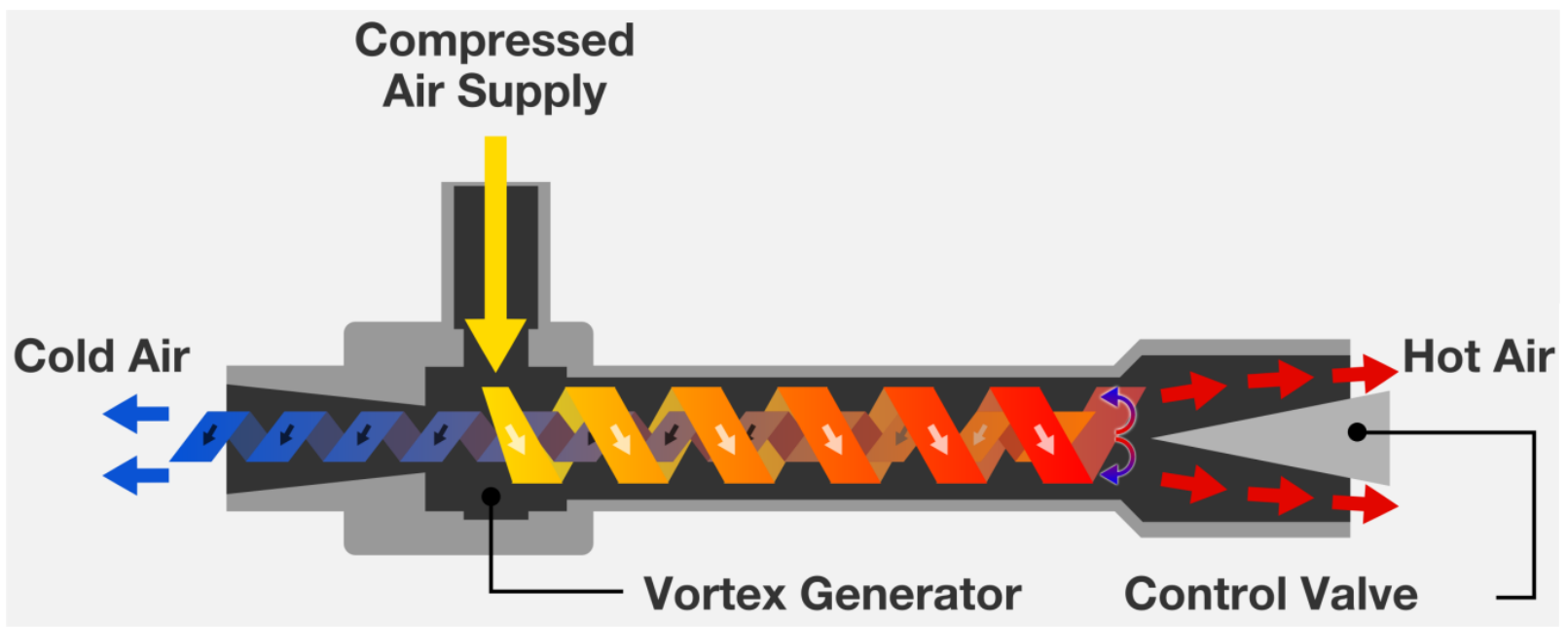

Figure 3-12: Schematic of vortex tube used in cooling system of the experimental facility (Airtx, 2016) 
The spinning chamber admits the compressed air and causes it to rotate. A vortex is formed and a pressure gradient establishes inside of the tube, forcing the high velocity and high temperature air to the outside and the low velocity and low temperature air to the inside. The stream is then split by a conical nozzle, with the high temperature stream being ejected at one end and the low temperature stream being ejected at the opposite end of the tube. The fraction of the two streams can be adjusted using a control valve. This allows to regulate the mass flow rate and temperature of both streams (reducing the cold stream temperature simultaneously reduces the cold stream mass flow rate and vice versa). Since each vortex tube can only provide a certain amount of coolant, a system comprising of four Exair Corporation vortex tubes (each able to handle approximately $85 \mathrm{~g} / \mathrm{s}$ of air) in parallel operation is used. This results in a total coolant mass flow rate of roughly $230 \mathrm{~g} / \mathrm{s}$ when the vortex tubes are set to $70 \%$ cold mass flow fraction. This amount is required to cool the three nozzle guide vanes and endwalls.

The schematic overview and a photo of the cooling system's setup are shown in Figure 3-13. Compressed air is supplied by the 27.6 bar tanks (1). The air's pressure is dropped to 13.8 barg (maximal operating pressure of vortex tube is 17.2 bar) by a high-pressure regulator (2). The flow enters (3) the inlet manifold (4) in order to uniformly feed the four vortex tubes (5). The hot stream of the vortex tubes is ejected into the atmosphere while the tubes' cold stream is collected in the coolant plenum (6). The surplus valve (7) keeps the pressure of the coolant plenum constant (valve opens when pressure exceeds 2 barg). The coolant flow's pressure is finally dropped to 0.7 barg by the low pressure regulator (8) and is transmitted to the working section via plastic tubing (9). The entire cooling system and tubing is insulated (although not shown) to minimize the heat pickup between the coolant plenum and the working section. 

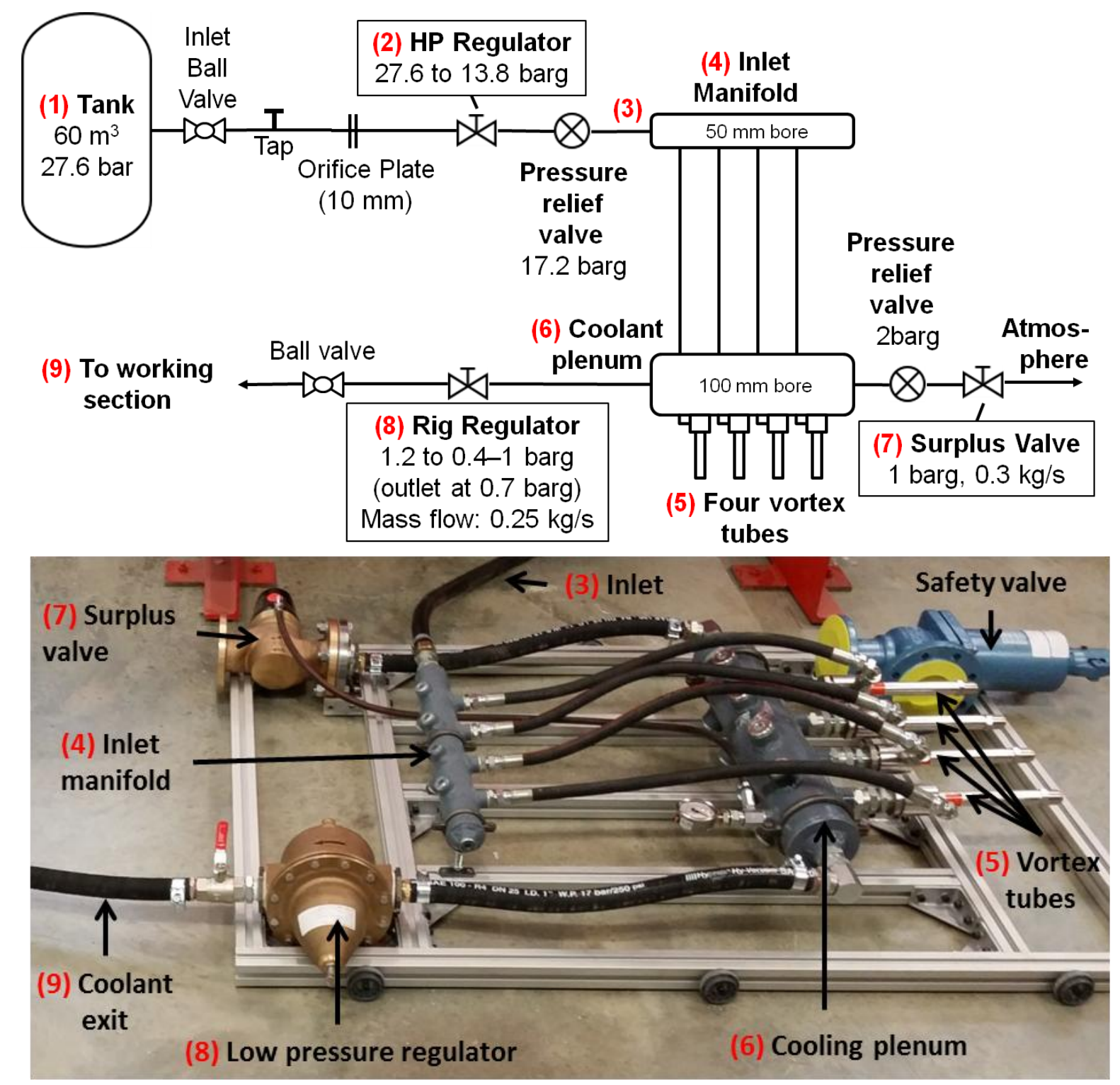

Figure 3-13: Schematic overview (top) and photo (bottom) of the cooling system for the experimental facility 


\section{Chapter 4: Numerical Methodology}

\subsection{Abstract}

This chapter will introduce the numerical methodology used in the study. Firstly, the in-house flow solver TBLOCK will be briefly described. Subsequently the conventional and integrated vane domains will be illustrated for a linear setup and an annular full-stage setup. Finally, information on the boundary conditions will be supplied.

\subsection{Flow solver}

All numerical simulations in this study were performed on the CFD in-house code TBLOCK. TBLOCK is a flow solver developed by Denton (1983) and is widely used in the turbomachinery community. It solves the unsteady, compressible, threedimensional governing equations in a cylindrical coordinate system using the finite volume method with cell corner storage of the flow variables. The multiblock nature of the code allows the efficient treatment of complex geometries. To reduce the computation time and enable large-scale computations the code was parallelised using MPI. Different LES models were implemented by Klostermeier (2008). To march the flow forward in time, TBLOCK offers an explicit scheme and the implicit dual time stepping algorithm. The mixing length (ML) approach is chosen for turbulence modelling for its simplicity and good agreement to test cases that have been widely demonstrated (Denton \& Pullan, 2012). It allows the tuning of two parameters - namely the free stream turbulence level and the free stream mixing length - which were calibrated against experimental measurements for this study. The heat transfer prediction capabilities and different temperature wall functions, 
which were validated against experimental measurements conducted on the same cascade used in this work, were added by Mazzoni et al. (2015). Heat transfer coefficient distributions are obtained by comparing the difference in wall heat flux of two iso-thermal simulations (with differing wall temperatures). The heat transfer simulations do not incorporate a transition model such that results in the leading edge region may not be accurate.

\subsection{Numerical cases}

In this work, two different sets of simulations were performed. Firstly, steady CFDsimulations were performed for linear domains to compare the aerothermal and cooling performance of the conventional and integrated vanes. Secondly, unsteady annular full-stage simulations were used to determine the influence of the integrated combustor vane concept on the rotor and stage efficiency.

\subsubsection{Steady CFD for linear domain}

The steady CFD-simulations for the linear domains of the integrated and conventional vanes can be seen in the schematic of Figure 4-1.

a) Conventional vane (CV)

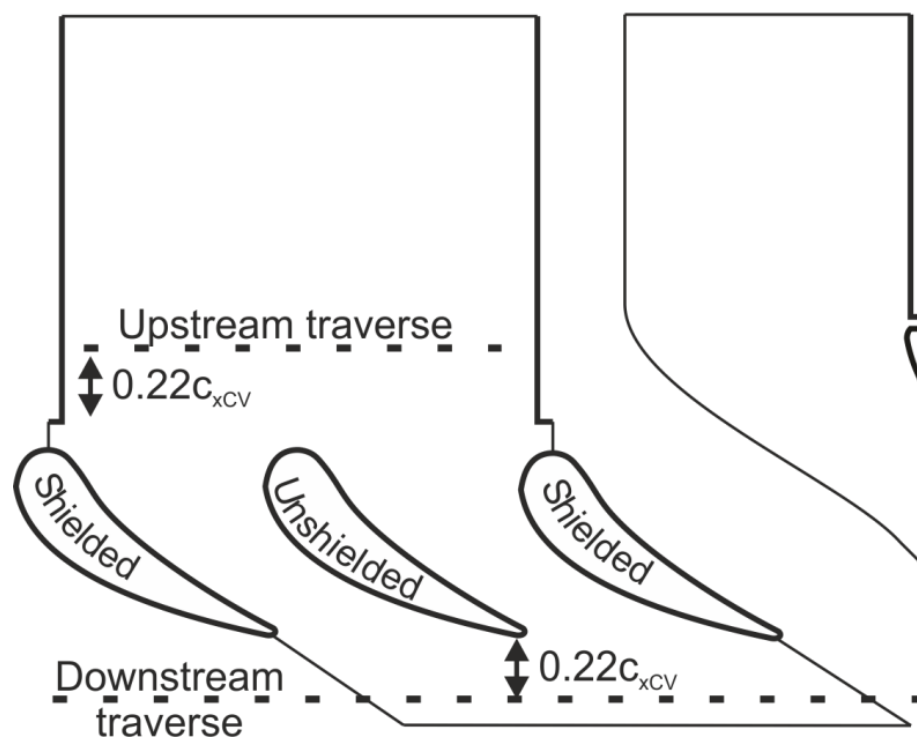

b) Integrated vane (IV)

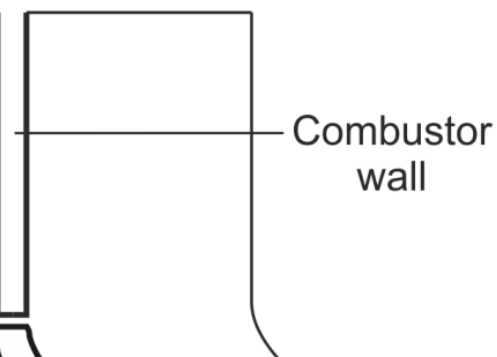

Figure 4-1: Schematic of steady CFD domain for conventional (a) and integrated (b) vane design 
Since the integrated vane design aims to achieve the same turning as the conventional vane design with half the number of vanes, the axial chord length had to be increased. The axial position of the trailing edge is kept identical in order to not prolong the engine. The integrated vane leading edge is thus moved further upstream into the transition duct. Moreover, the trailing edge thickness is kept constant to allow a fair comparison of losses. Structured meshes were obtained using ANSYS ICEM. The relatively simple geometry allows to use the H-grid approach. Mesh independence studies were conducted and resulting meshes consist of 4.3 million and 5.6 million nodes for the conventional and integrated vane domains respectively. The pitchwise node count is between 110-140 and the spanwise node count is 90 . Figure 4-1 also indicates the axial position of the upstream and downstream aerodynamic traverse measurements presented in the study.

Three different inlet boundary conditions were applied to represent a no-swirl (representative of the upstream turbulence grid), a swirl and a reversed swirl inlet scenario. At the inlet, two-dimensional total pressure profiles were used together with pitch and yaw angle distributions (set to 0 for no-swirl). These were obtained from aerodynamic traverse measurements downstream of the combustor transition duct (see 'upstream traverse' in Figure 4-1). The total pressure distribution for the no-swirl case is displayed in Figure 4-2. The boundary layer on the hub and casing endwall, as well as on the combustor walls can be seen. The graph shows the total pressure along a horizontal (pitchwise) and vertical (spanwise) cut. The total pressure is nearly constant at 1.6 bar in the mainstream (i.e. between $5 \%-95 \%$ of span and pitch) and drops towards the walls. An accurate inlet boundary condition is important for an accurate prediction of aerodynamics and heat transfer in the first vane passage, as shown in the introduction. Similar measurements (including pitch and yaw profiles) for the swirl and reversed swirl cases will be presented later in the thesis (see Figure 
8-1). The outlet of all three cases is specified as a constant static pressure boundary condition.

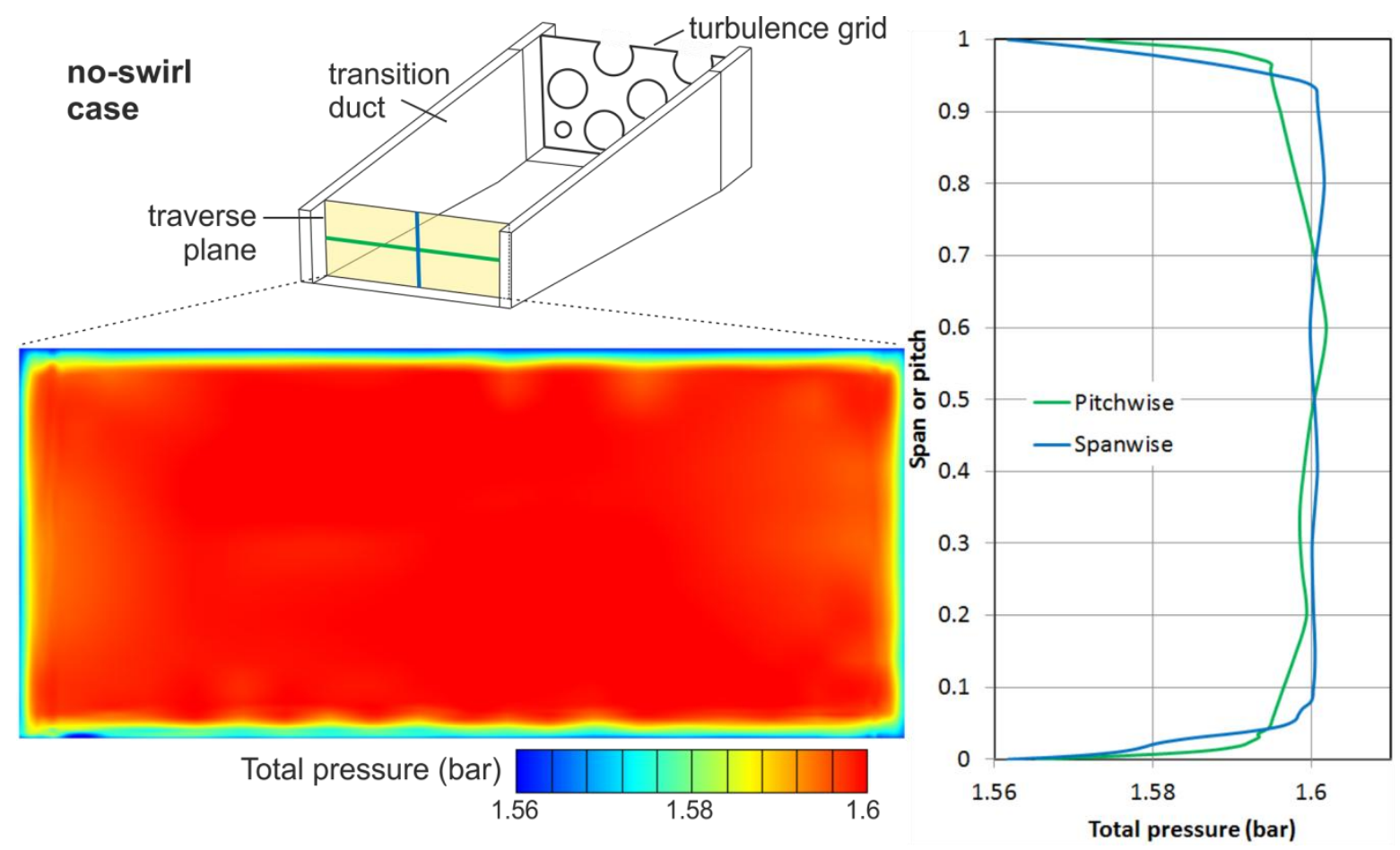

Figure 4-2: Total pressure at outlet of combustor transition duct obtained from experimental traverse measurements with upstream turbulence grid (no-swirl case)

\subsubsection{Unsteady CFD for annular full-stage domain}

Finally, CFD-simulations were performed for annular stage geometries for the conventional and integrated vanes in order to investigate the concept's potential for real-engine application and the effects on the turbine rotor. The conventional and integrated vanes were thus scaled up to the real engine size (the linear cascade is a $60 \%$ model) and converted from a linear into an annular geometry. The rotor used for all full stage simulations is from the MHI F-type gas turbine, and its geometry, provided by the industrial partner, can be seen in Figure 4-3. The rotor inlet metal angle is smallest at the casing since the circumferential rotor velocity is highest here (because of highest radius and constant rotational speed). It is noted that this rotor 
was designed for the application with conventional vanes, and using it for integrated vane full stage simulations represents a conservative approach. Moreover it is noted that the simulations in this thesis do not include the modelling of the rotor tip leakage flow for simplicity reasons.

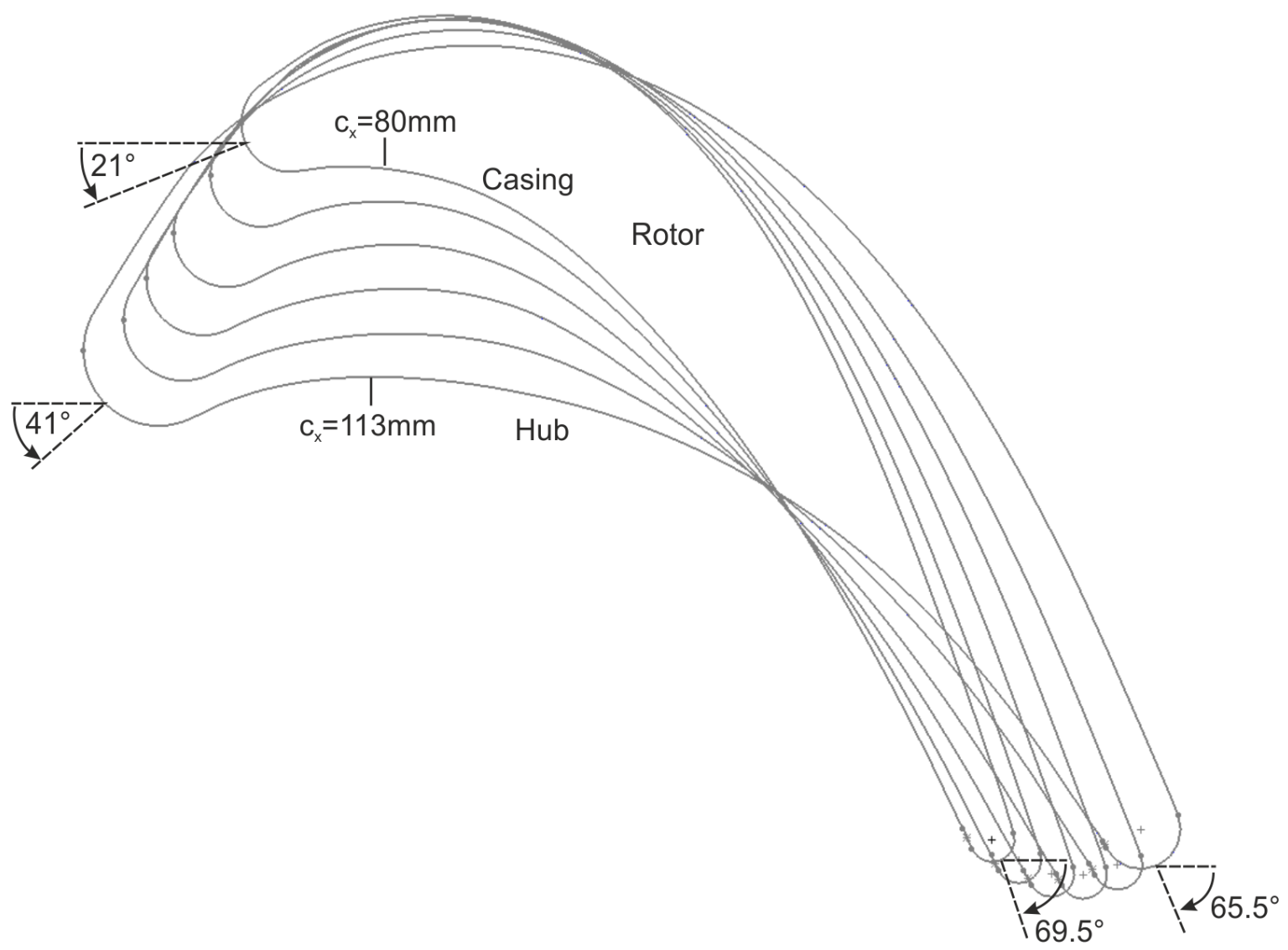

Figure 4-3: Geometry of high-pressure turbine rotor used for full stage simulations

The flow domain investigated for the full stage simulations consists of combustor transition duct, stator vanes and rotor. The ratio of the number of rotors to combustors is 5 to 1 and the annular-stage domains represent $18^{\circ}$ of the annulus. Figure 4-4 shows the annular-stage domain for the integrated vane, the conventional vane, and a section of the mesh at the integrated vane stator-rotor interface. A corresponding profiled integrated vane stage domain was also designed. The meshes have 14.2 million and 14.3 million nodes for the conventional and integrated vane design respectively. The simulations used a total pressure inlet, a static 
pressure outlet and a rotor rotational speed of $3000 \mathrm{rpm}$ representative of real engine conditions. More detailed information on the numerical setup can be found in Table 4-1. For the swirl case, the full-stage simulations used the total pressure distribution obtained through the traverse measurements (downstream of the combustor transition duct with upstream swirl generator) scaled up to 16.4 bar and identical pitch and yaw distributions. A mixing plane model was used for steady full stage calculations, and sliding interfaces were used for unsteady full stage calculations. The unsteady simulations were initiated from a converged steady solution and run for 60 rotor blade passes so that fully periodic aerodynamic behaviour was obtained.

\begin{tabular}{|c|c|}
\hline Condition & Value \\
\hline Inlet total pressure & $16.4 \mathrm{bar}$ \\
\hline Outlet static pressure & $8.7 \mathrm{bar}$ \\
\hline Inlet total temperature & $1690 \mathrm{~K}$ \\
\hline Rotor rotational speed & $3000 \mathrm{rpm}$ \\
\hline Duct inclination in respect to $\mathrm{3}$ & $4^{\circ}$ \\
\hline Computational domain & 1 duct; $2 \mathrm{CV} / 1 \mathrm{IV} ; 5$ rotor blades \\
\hline Domain periodicity & $1 / 20$ th of annulus $\left(=18^{\circ}\right)$ \\
\hline Axial chord at midspan & $\mathrm{CV} / \mathrm{IV} /$ Rotor $=140 / 260 / 96 \mathrm{~mm}$ \\
\hline Mesh size & 14 million \\
\hline Turbulence model & Mixing length \\
\hline Stator-rotor interface & Sliding plane \\
\hline
\end{tabular}

Table 4-1: Numerical setup of full-stage simulations 

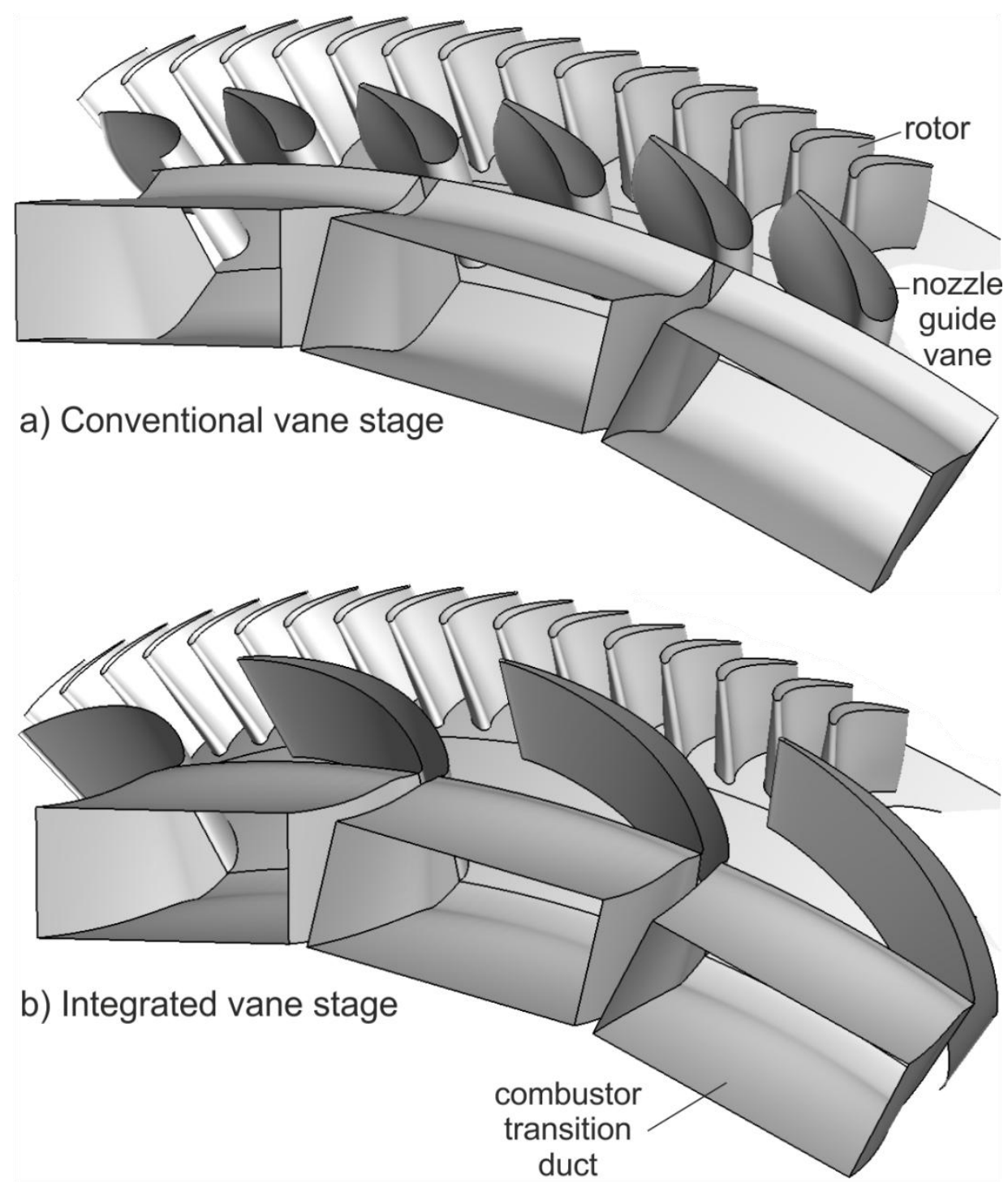

c) Integrated vane stage mesh at stator-rotor interface

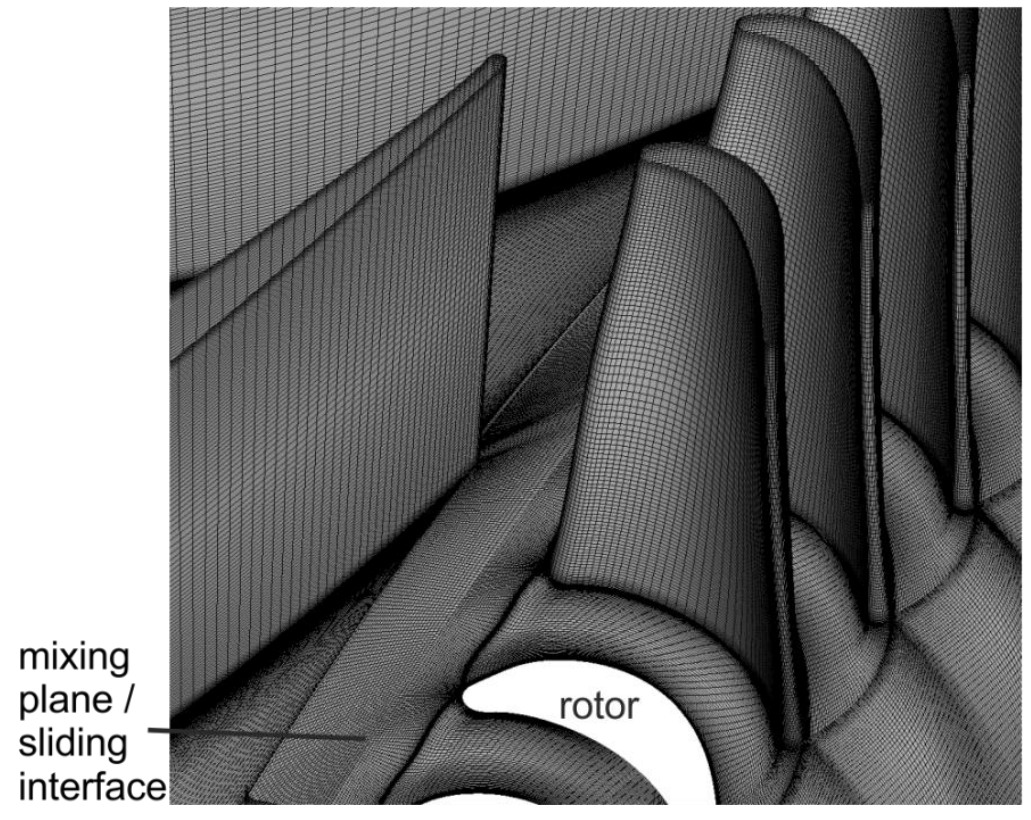

Figure 4-4: Annular full stage domain of conventional vane (a) integrated vane (b) and mesh at stator-rotor interface (c) 


\section{Chapter 5: Design and Aerodynamic Performance of Integrated Vane}

\subsection{Abstract}

This chapter focuses on the design and aerodynamic performance of the integrated vane. Two numerical parametric studies are performed to determine the integrated vane's profile. The first study explains the effects of three-dimensional vane profiling (such as sweep and lean) on the flowfield inside and downstream of the vane passage. The second study highlights the benefits of shifting the loading distribution in the axial direction. The two studies suggest a suitable integrated vane profile which is subsequently manufactured and tested in the linear cascade. A good agreement is found between numerical predictions and experimental measurements and a comparison is drawn with the conventional vane's aerodynamic performance. Finally, a reduction in loss of the integrated vane compared to the conventional vane design is illustrated and explained by changes in the profile and endwall loss generation. An overview of the results presented in this chapter is given by Jacobi and Rosic (2015).

\subsection{Design of integrated vane}

The conventional as well as integrated vane domains shown in Figure 4-1 are of identical axial length. This is achieved by axially moving the integrated vane, which has a $78 \%$ longer axial chord and $7 \%$ smaller solidity (ratio of true chord to pitch) than the conventional vane, further into the transition duct. This helps to avoid a enlongation of the engine and allows for a fair comparison. The latter is also facilitated by identical trailing edge thickness of conventional and integrated vanes, which further ensures that the trailing edge internal cooling geometry can be implemented in the same way as for a conventional vane design. 
The development of the integrated vane was started with the goal to achieve the same turning as the conventional vanes while ideally reducing aerodynamic loss. A comprehensive numerical parametric study was undertaken to investigate the benefits of three-dimensional geometry variations such as blade sweep and lean.

Figure 5-1 shows the schematic of these three geometries. The two-dimensional vane has the same profile at every span position and includes fillets at the endwalls. The fillets' effect on aerodynamic performance was found to be marginal. The vane with sweep has the same profile as the two-dimensional vane and only a slightly extended midspan section. The vane with lean features a slightly reduced metal exit angle towards the endwalls and slightly increased metal exit angle towards the midspan.

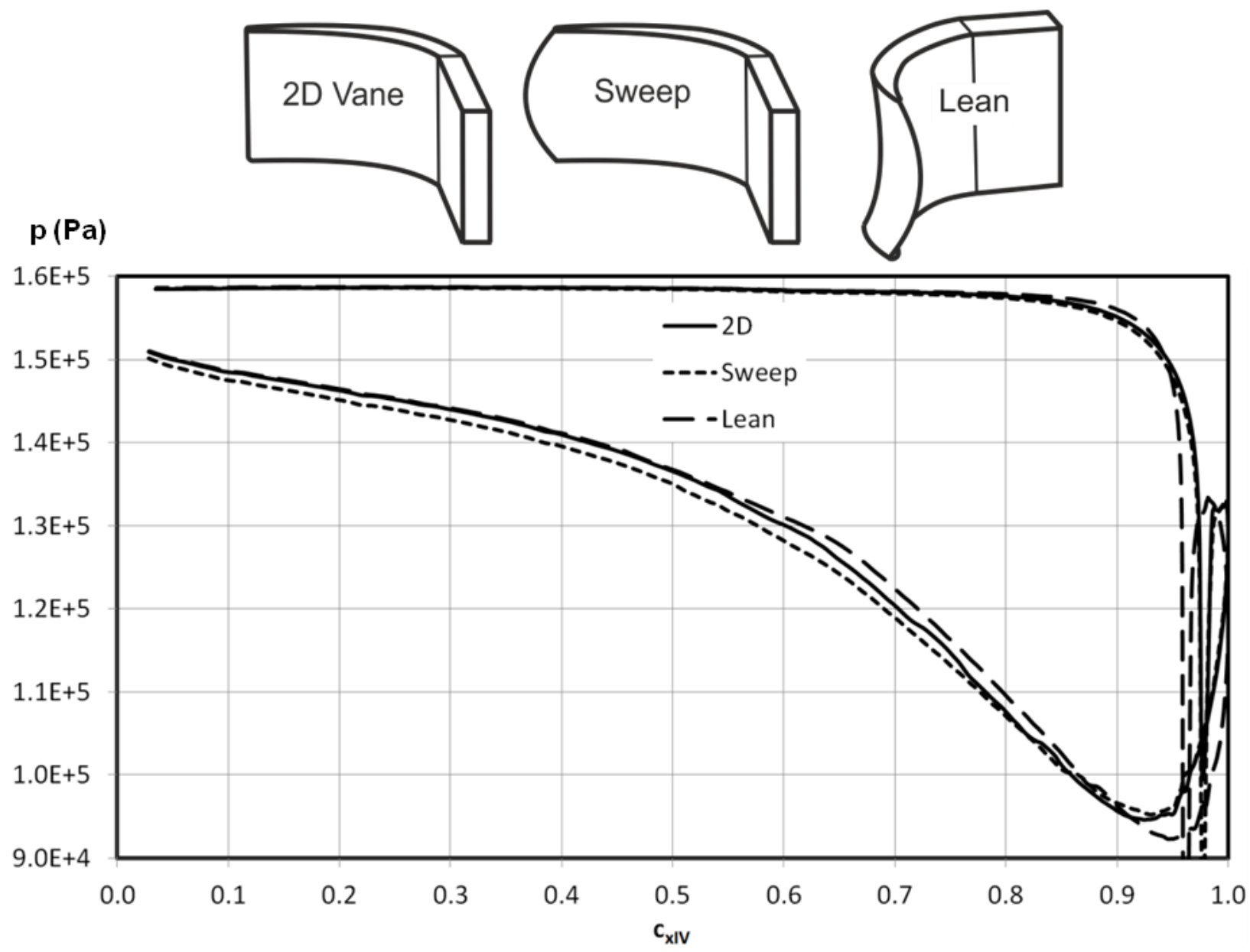

Figure 5-1: Predicted static pressure around integrated vane's midspan for three geometries 
Moreover, Figure 5-1 shows the predicted static pressure distribution along the integrated vane's midspan. The pressure stays nearly constant on the pressure side until $90 \%$ of the axial chord after which the flow is quickly accelerated. On the suction side, the flow is accelerated nearly linearly until $50 \%$ of the axial chord after which the pressure drops quicker reaching its minimum between $90 \%-95 \%$ of the axial chord. At the trailing edge the solver fails to predict the expected pressure distribution ('fishtail'). It can be seen that the two-dimensional vane and the vane with sweep have a nearly identical loading distribution (only slightly offset due to the swept vane's slightly longer axial chord). The vane with lean shows more abrupt flow acceleration on the pressure side and a lower pressure minimum on the suction side at a slightly later axial chord position, indicating the increased loading due to the increased metal exit angle.

In order to obtain a better understanding of the flowfield in the integrated vane passage, Figure 5-2 shows the predicted streamtraces on the integrated vane suction surface and endwall for the three geometries. The streamtraces of the twodimensional integrated vane contract on the suction surface towards the trailing edge. This is caused by the migration of the low-momentum endwall fluid (that is overturned within the passage by the pitchwise pressure gradient) onto the suction surface. The flow at midspan stays attached until the trailing edge while the secondary flow close to the endwalls separates from the suction surface towards the trailing edge. The vane with sweep shows a very similar distribution since the vane profile stays identical. A slightly enlarged casing secondary flow separation is evident, and the flow at midspan does not stay fully attached until the trailing edge due to the prolonged chord length (and thus longer boundary layer diffusion). 
a) Two-dimensional vane

midspan flow stays

attached until TE
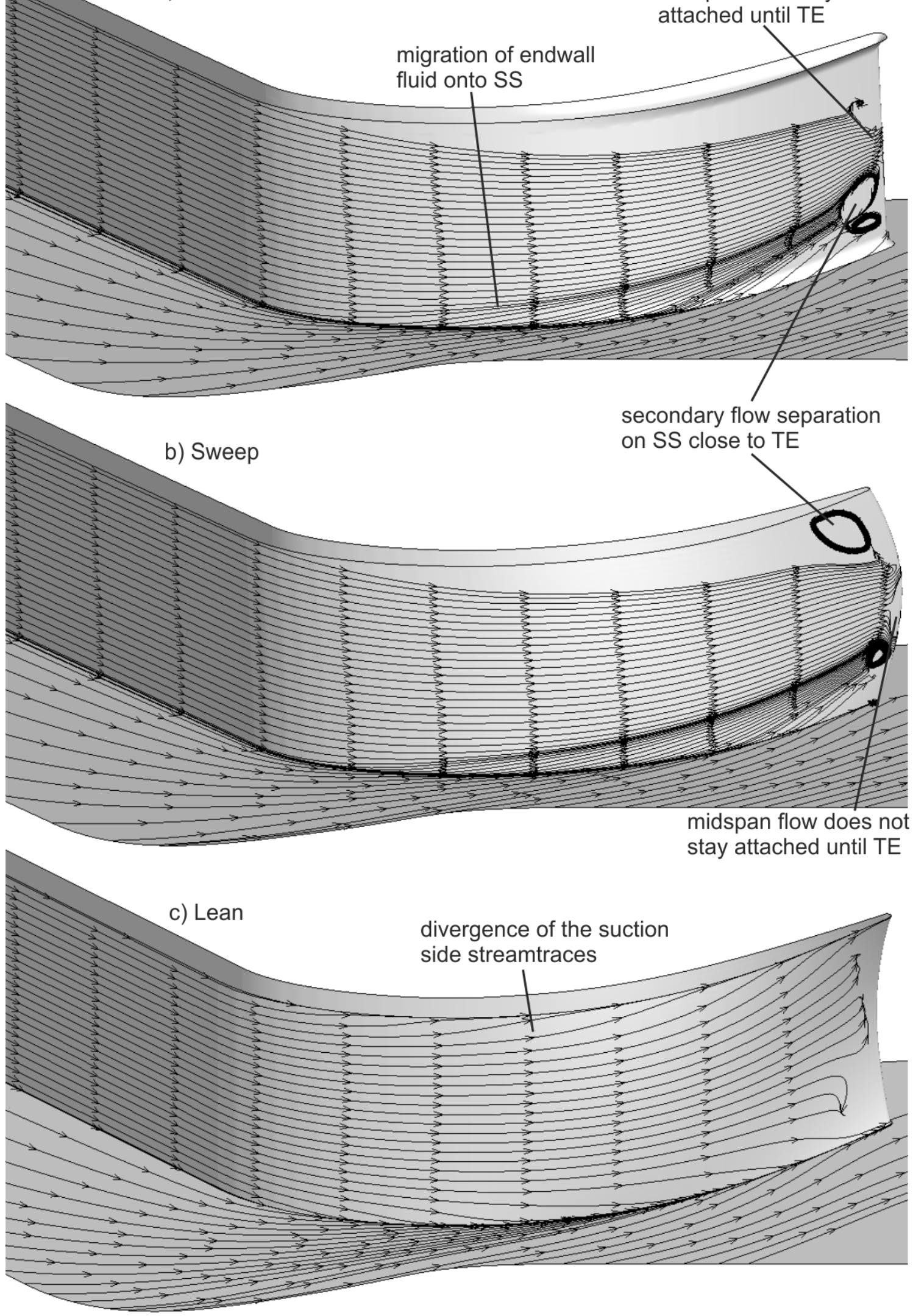

Figure 5-2: Predicted streamtraces on the integrated vane suction side and endwall for three geometries 
The vane with lean shows a distinctively different distribution. The streamtraces diverge on the suction surface. The increased loading at midspan (and thus higher static pressure) incentivises the flow to radially convect to spanwise positions of lower vane turning (and thus lower static pressure). The reduced vane turning towards the endwalls further lowers the overturning of the endwall fluid and thus its potential migration onto the suction surface. The flow on the suction surface does not stay fully attached until the trailing edge but a region of strong separation is not evident. Now that a better understanding of the flow within the passage is established, further investigations will focus on the downstream aerodynamic performance of the integrated vane.

The plot in Figure 5-3 shows the total pressure (non-dimensionalised by upstream total pressure in transition duct) $\overline{p_{0}}$ downstream of the integrated vane for the three selected geometries. The two-dimensional integrated vane results in a symmetric wake with very similar loss core distribution (at $20 \%$ and $70 \%$ of span) and levels. The wakes of the vane with sweep and lean are more skewed. The vane with sweep results in increased profile loss (due to the increased chord length at midspan) and a stronger casing loss core that migrated closer to the casing endwall (at $90 \%$ of span). This could be expected from the previous streamtrace distributions. The vane with lean shows a reduced radial migration of the secondary flow due to the reduced vane metal exit angle at the endwalls and the divergence of the streamtraces seen on the suction surface. 

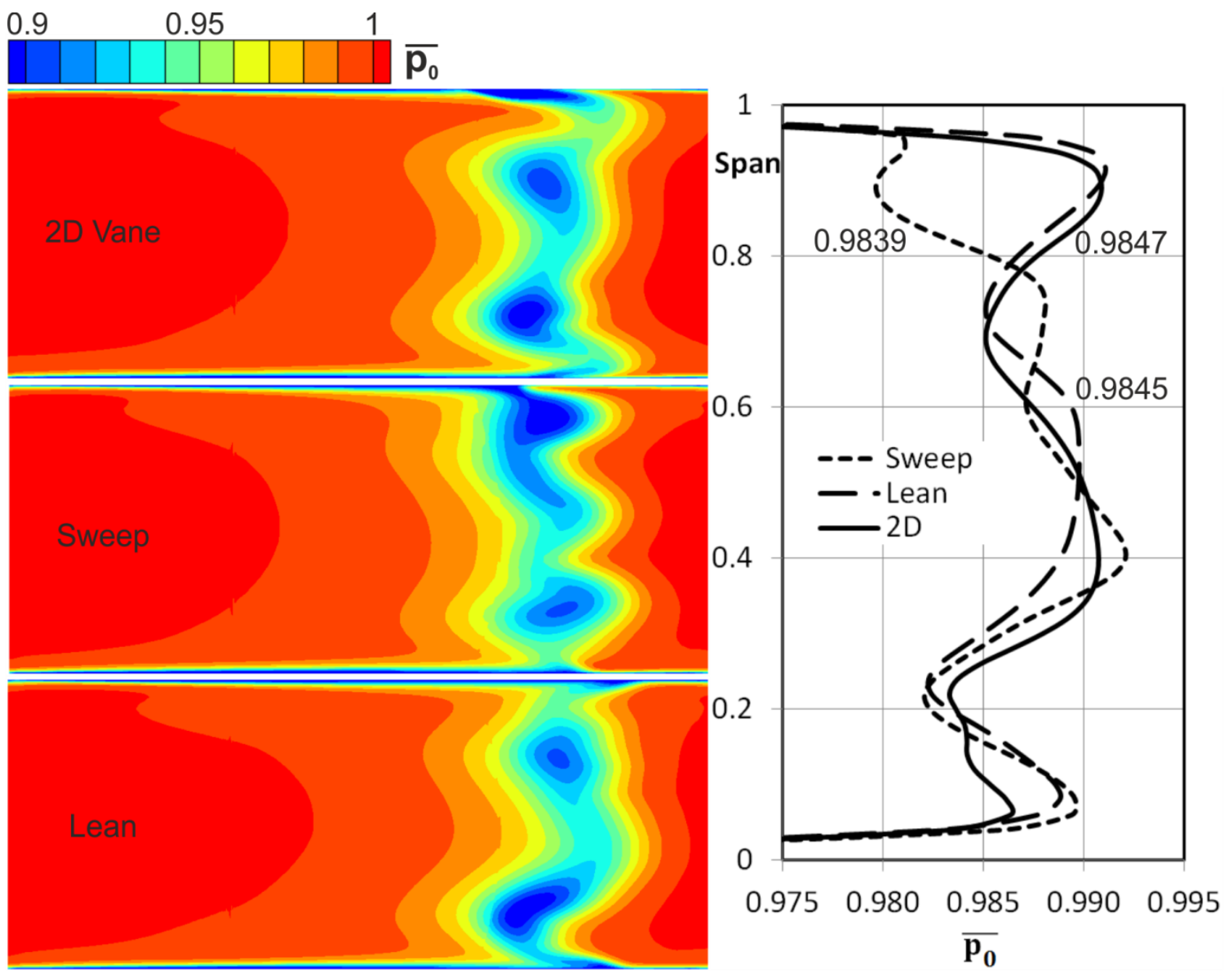

Figure 5-3: Predicted effect of three-dimensional geometry variations on dimensionless total pressure downstream of integrated vane; numbers represent averaged values

Figure 5-4 similarly shows the turning downstream of the integrated vane for the three vane profiles. The two-dimensional integrated vane shows nearly symmetric distributions (as expected), with overturning close to the endwalls and a region of underturned flow on the vanes pressure side (to the right of the wake). The vane with sweep leads to reduced turning close to the endwalls due to the shorter chord length in this region. The vane with lean results in increased turning in the midspan region due to the increased vane metal exit angle at midspan, and decreased turning close to the endwalls (due to the decreased vane metal exit angle at the endwalls). Furthermore a small region of very low turning can be seen close to the casing 
endwall of the vane with lean. The low momentum fluid is turned so strongly that it leads to slightly negative axial velocities.
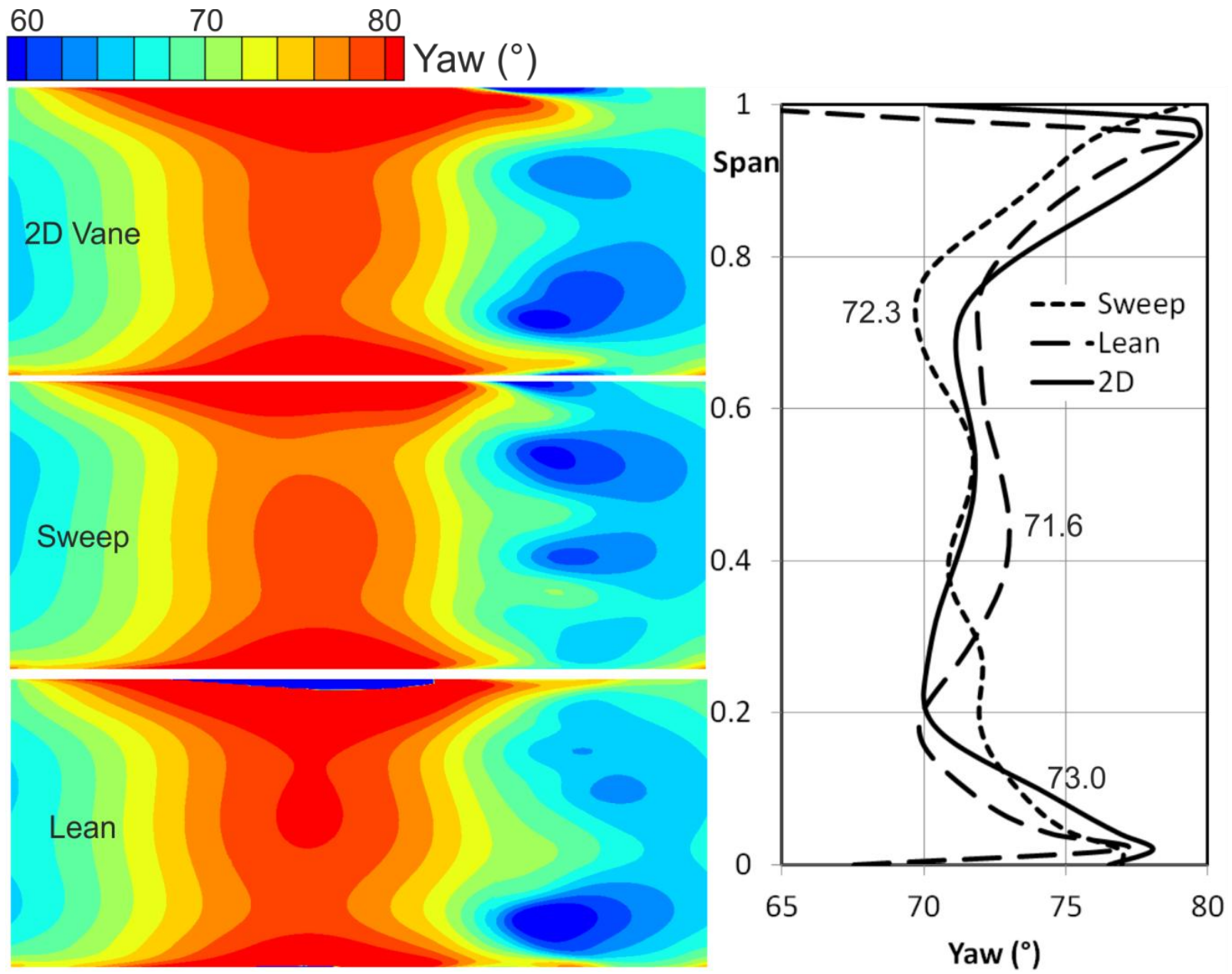

Figure 5-4: Predicted effect of three-dimensional geometry variations on turning downstream of integrated vane; numbers represent averaged values

Averaging the distributions of Figure 5-3 and Figure 5-4 shows the highest turning of $73.0^{\circ}$ (72.3 $3^{\circ}$ with sweep and $71.6^{\circ}$ with lean) and the lowest dimensionless total pressure loss of $1.53 \%$ (1.61\% with sweep and $1.55 \%$ with lean) for the twodimensional vane. It was thus decided to choose the two-dimensional vane as the best and simplest starting design choice. It should furthermore be noted that these results suggest that conventional vane profiling wisdom is only partially applicable due to the alteration of the overall vane passage geometry. The integrated vane passage now exhibits an apparent resemblance of a box doing the turning. 
A further study was performed to investigate the influence of a changed loading distribution on the vane's aerodynamics. Figure 5-5 shows the predicted static pressure distribution around the integrated vane's midspan for four different integrated vane designs, with 'Frontloaded 0' (representing the two-dimensional vane obtained in the last parametric study) being the most aft-loaded and 'Frontloaded 3' being the most front-loaded.

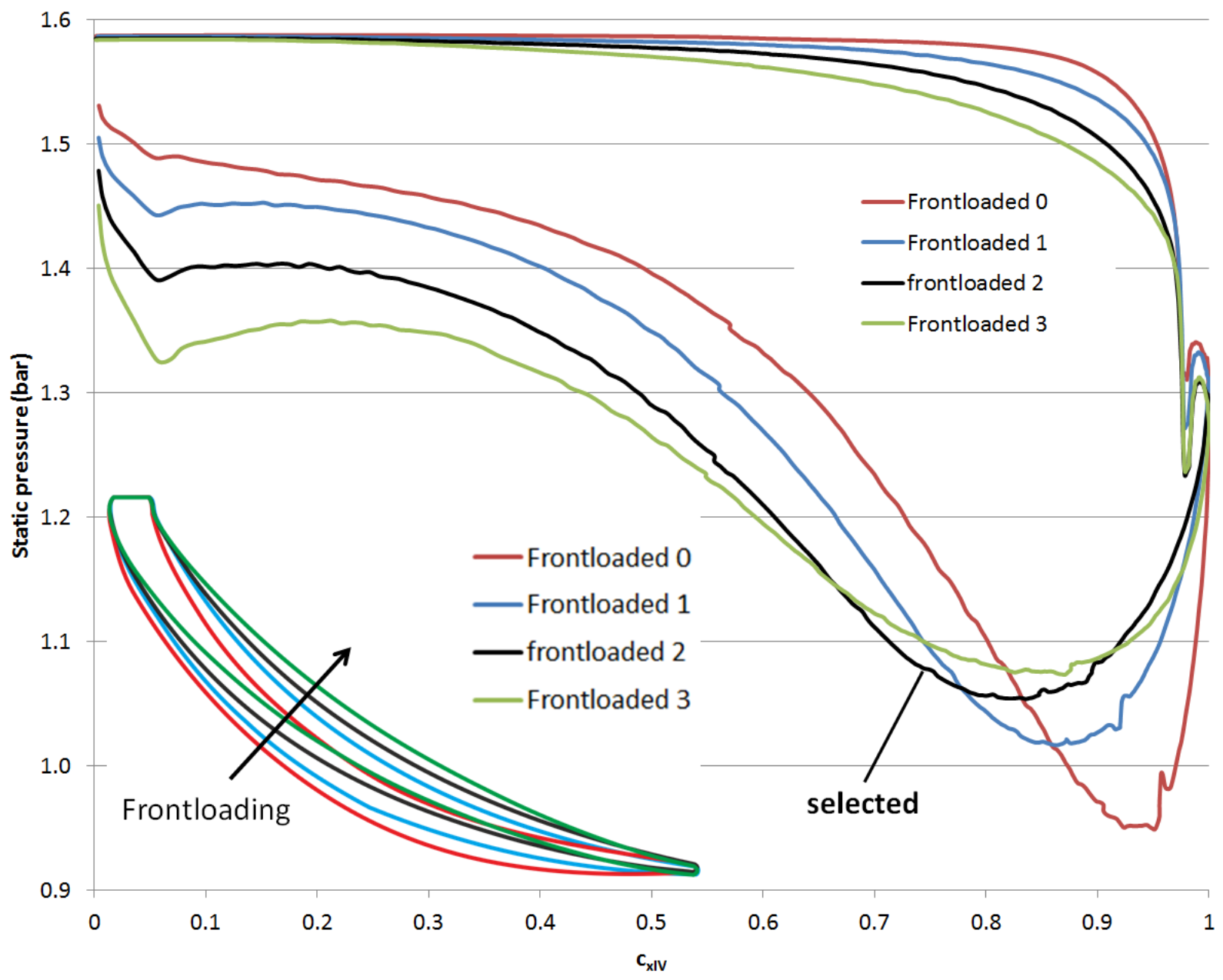

Figure 5-5: Predicted effect of frontloading on the midspan static pressure distribution of the integrated vane

It can be seen that frontloading the vane leads to an increased flow velocity on the suction side in the first approximately $75 \%$ of axial chord, and to an increased minimum static pressure (thus lower peak velocity) at a slightly earlier axial location. The flow acceleration on the pressure side also occurs earlier and is thus less abrupt. 
By frontloading the vane, the turning is thus distributed more evenly across the vane and leads to a decreased risk of boundary layer separation on the suction surface. The latter is to be avoided at all costs as it leads to a poor performance. On the other hand, frontloading the integrated vane will increase the strain on the combustor walls and lead to an increased pressure gradient in the gap between the combustor wall trailing edge and integrated vane leading edge which can cause difficulties for cooling the integrated vane pressure surface (see chapter 'cooling').

In order to evaluate the effect of frontloading on the pressure loss, Figure 5-6 shows the predicted pitchwise-averaged dimensionless total pressure downstream of the integrated vane for the four integrated vane designs. The aft-loaded (Frontloaded 0 \& Frontloaded 1) designs lead to the highest profile loss due to the high peak velocity on the suction side, and to the most pronounced loss cores with strong radial migration. The front-loaded (Frontloaded 2 \& Frontloaded 3) designs resulted in the lowest profile loss due to the lowest peak velocities (highest minimum static pressure) and the least pronounced loss cores. The 'Frontloaded 2' option (black line in Figure 5-5 and Figure 5-6) was finally selected based on the described trade-offs (risk of boundary layer separation, pronounced loss cores and high profile loss for aft-loaded vane; strain on combustor walls and cooling challenges for front-loaded vane). 


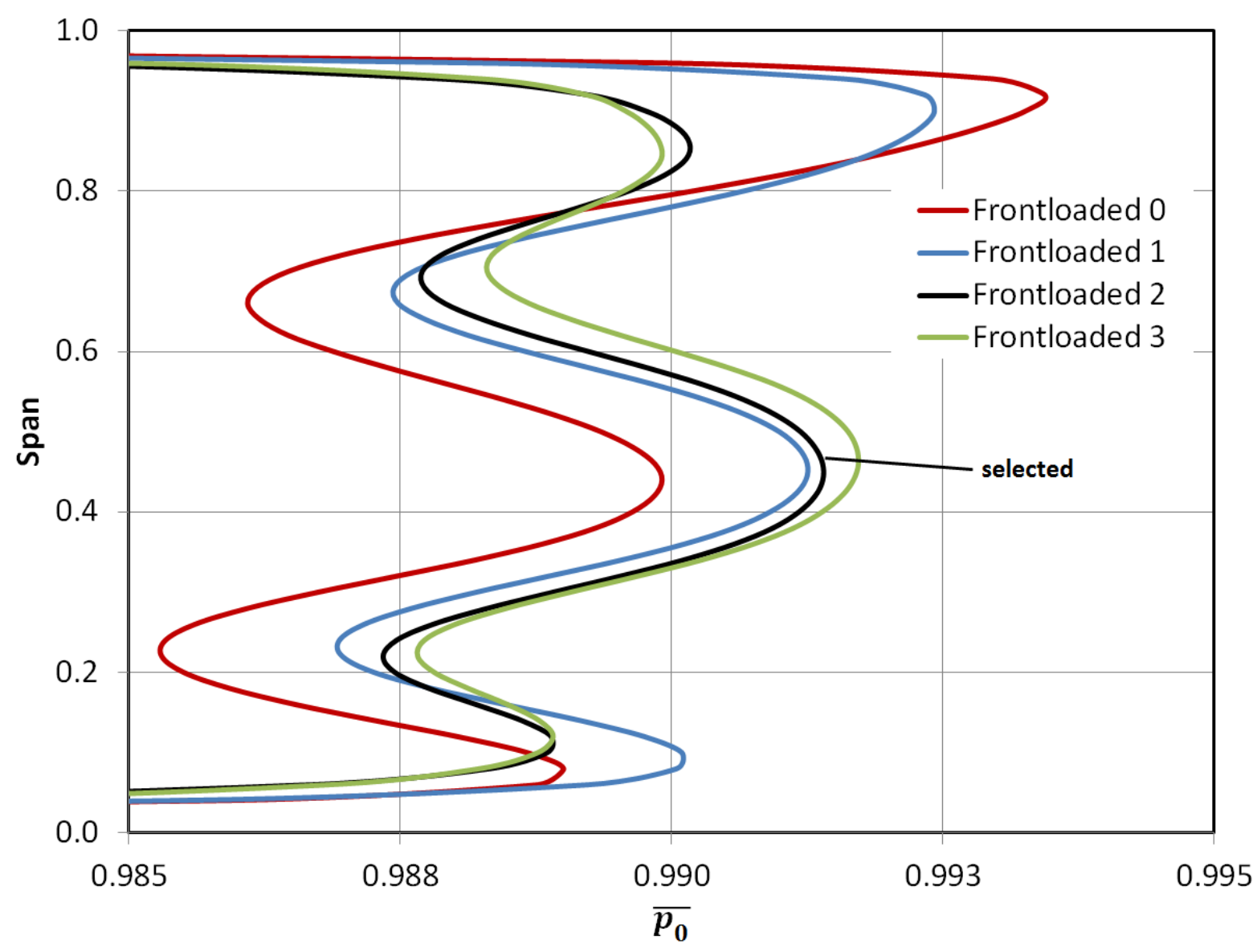

Figure 5-6: Predicted effect of frontloading on the pitchwise averaged total pressure downstream of the integrated vane

The predicted aerodynamic behaviour of the selected, final integrated vane is shown in Figure 5-7. It can be seen that the less aft-loaded design of this final integrated vane leads to the removal of the secondary flow separation on the vane's suction surface close to the trailing edge seen in Figure 5-2a. The dimensionless total pressure distribution downstream of the integrated vane shows a symmetric wake with two distinct loss cores that contain the low-momentum fluid from the upstream endwall boundary layer. The hub and casing boundary layers can still be seen downstream of the vane and reach across the whole pitch. The yaw distribution downstream of the integrated vane is symmetric as well, with a region of high turning close to the endwalls (located to the left of the wake) and a region of low turning located to the right of the wake. In order to validate the aerodynamic predictions of 
the numerical simulations, the final integrated vane was subsequently experimentally tested in the linear cascade.
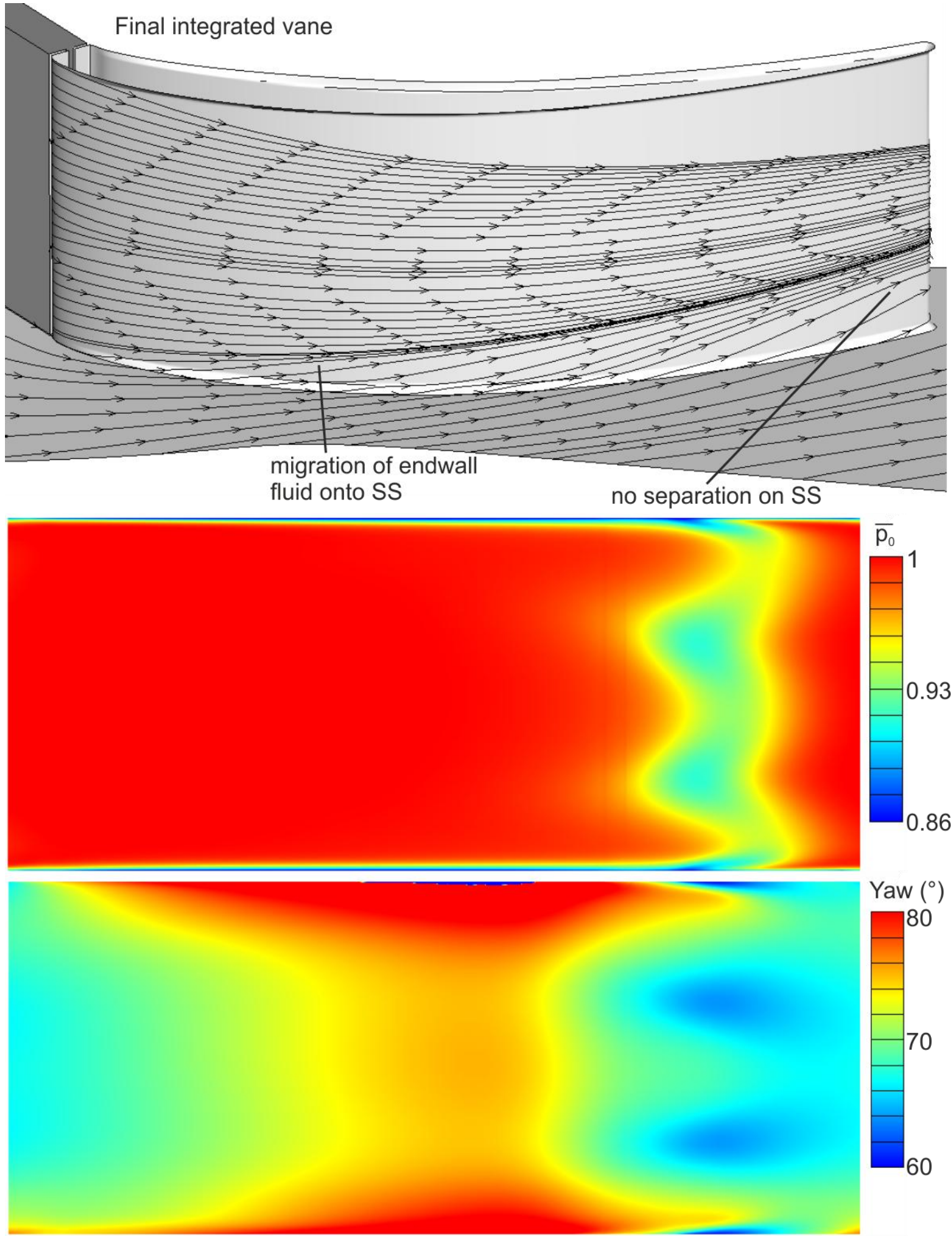

Figure 5-7: Predicted streamtraces on suction surface (top), dimensionless total pressure (middle) and yaw (bottom) distribution downstream of final integrated vane 


\subsection{Traverse measurements}

Aerodynamic measurements were conducted downstream of the vanes (location specified in Figure 4-1) for conventional and integrated vanes. Regions in the mainflow (distance from endwall $\geq 4 \mathrm{~mm}$ ) were measured by the 5 -hole-probe while regions in the boundary layer (distance from endwall $\leq 4 \mathrm{~mm}$ ) were resolved by the flat pitot-probe. Figure 5-8 shows total pressure (non-dimensionalised by upstream total pressure) $\overline{p_{0}}$ downstream of the conventional and integrated vanes.

a) Conventional vane
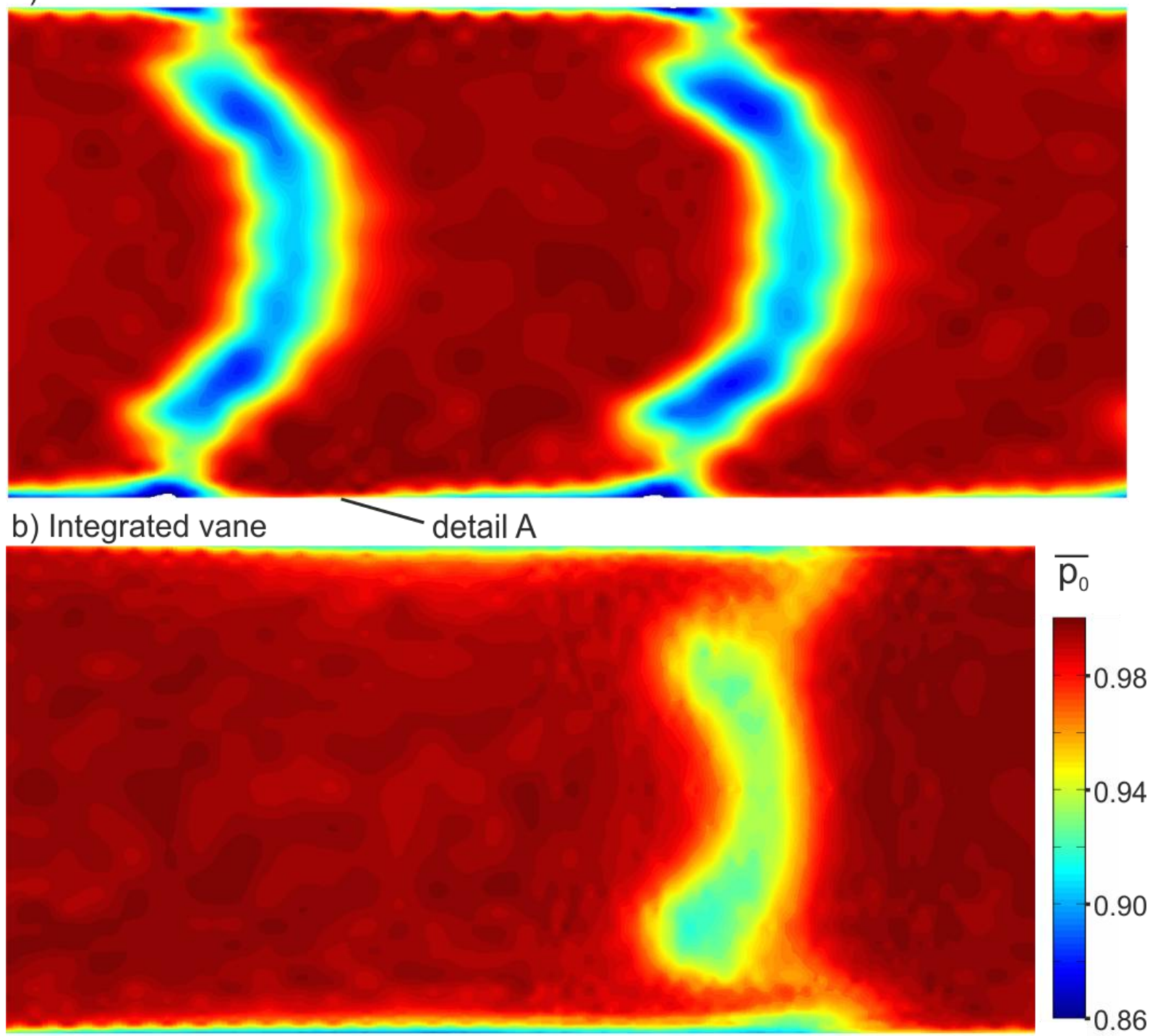

Figure 5-8: Aerodynamic traverse measurements showing dimensionless total pressure downstream of conventional (a) and integrated (b) vanes 
Since the integrated vane concept achieves the turning with one integrated instead of two conventional vanes, the number of wakes is halved. The integrated vane's wake is only slightly thicker than a single conventional vane's wake since the axial length (including transition duct wall and vane) of boundary layer growth and diffusion is similar. The contours furthermore show increased strength of secondary flow for the integrated vane. An increased radial migration of the loss cores can be seen which is due to the integrated vane's reduced aspect ratio and increased loading. Focusing on the endwall shows that for the conventional vanes there is an area of an extremely thin boundary layer towards the pressure side (to the right of the wake, see detail A). The integrated vane's boundary layer, in contrast, is thicker and reaches across the whole pitch.

Figure 5-9 similarly shows the turning downstream of the conventional and integrated vanes. It can be seen that the compound lean of the conventional vanes (with increased and reduced metal exit angle at midspan and at the endwalls respectively) leads to higher turning in the midspan region and lower turning in the endwall region. For the integrated vane, turning at midspan is less homogeneous due to the halved number of vanes. Furthermore, it can be seen that overturning at the endwalls is high for the integrated vane due to the increased vane metal exit angle and due to the thicker boundary layers compared to the conventional vanes. It is noted that the turning distributions resolve a smaller fraction of the actual span (approximately 94\%) then the total pressure distributions since the pitot probe (used in the endwall region) can only measure total pressure and is thus not able to capture flow angles. 
a) Conventional vane

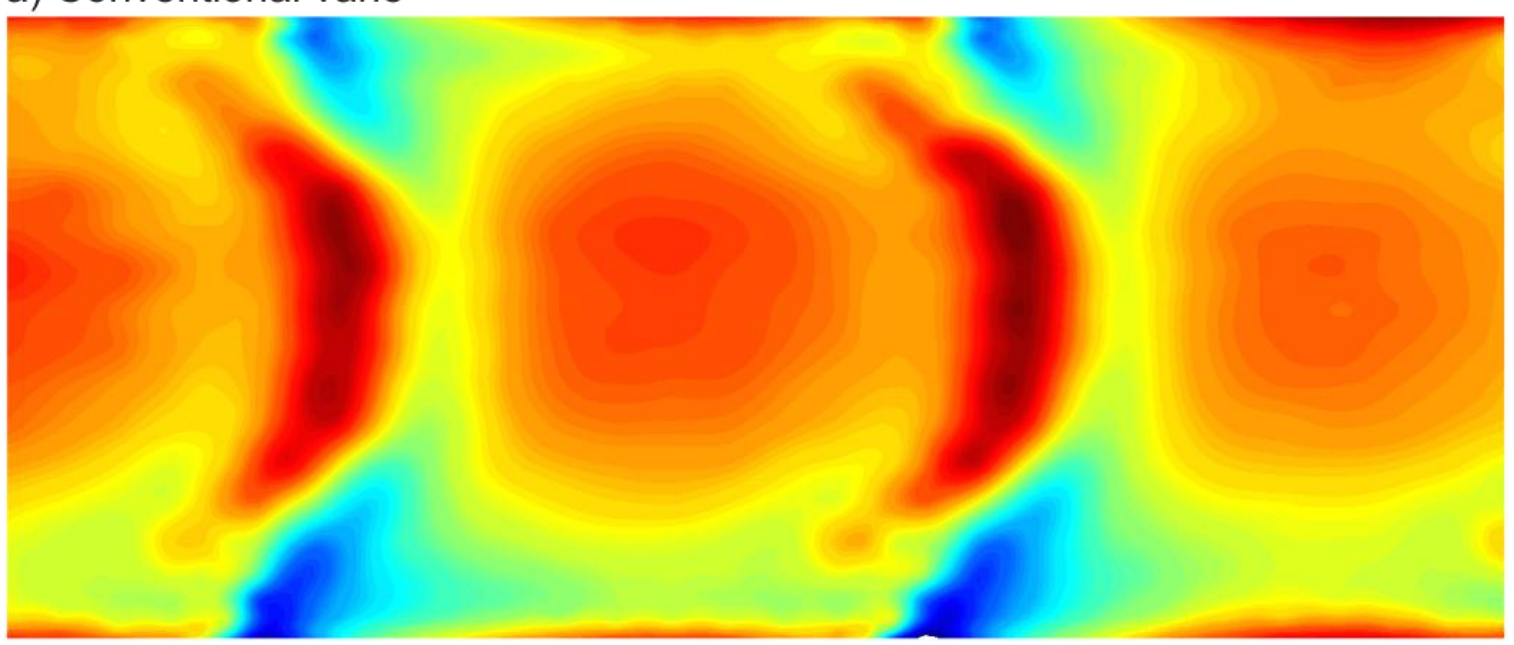

b) Integrated vane

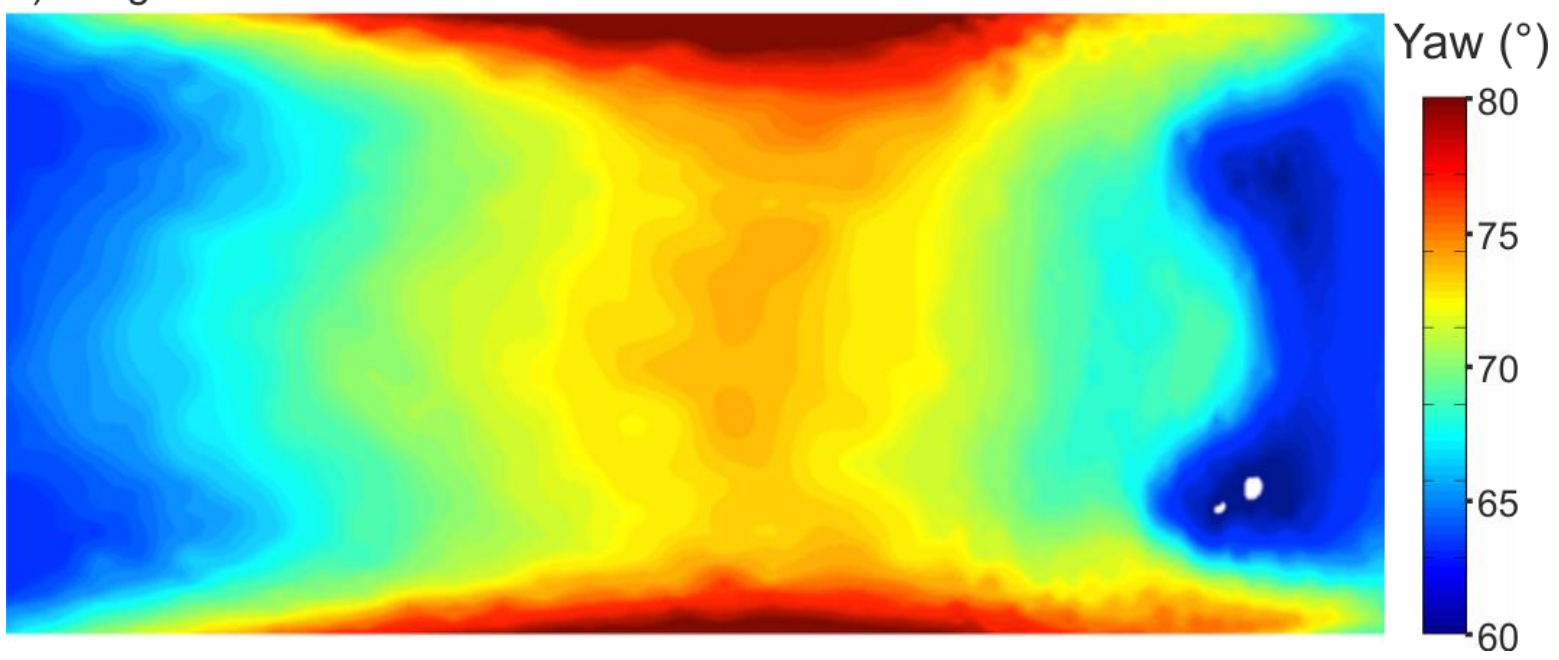

Figure 5-9: Aerodynamic traverse measurements showing dimensionless total pressure downstream of conventional (a) and integrated (b) vanes

Comparing the traverse measurements of the integrated vane's total pressure and yaw distributions to the numerical predictions in Figure 5-7 reveals a very good qualitative and quantitative agreement. Figure 5-10 furthermore compares the spanwise distribution of pitchwise-averaged dimensionless total $\overline{p_{0}}$ downstream of the vane trailing edges between experiments and CFD. The CFD-simulations of the conventional vanes underestimate the strength of secondary flows. Overall, qualitative and quantitative agreement is found to be good for both cases. A distinct overall increase of dimensionless total pressure of the integrated vane compared to the conventional vanes is apparent, already indicating a reduction in loss. 

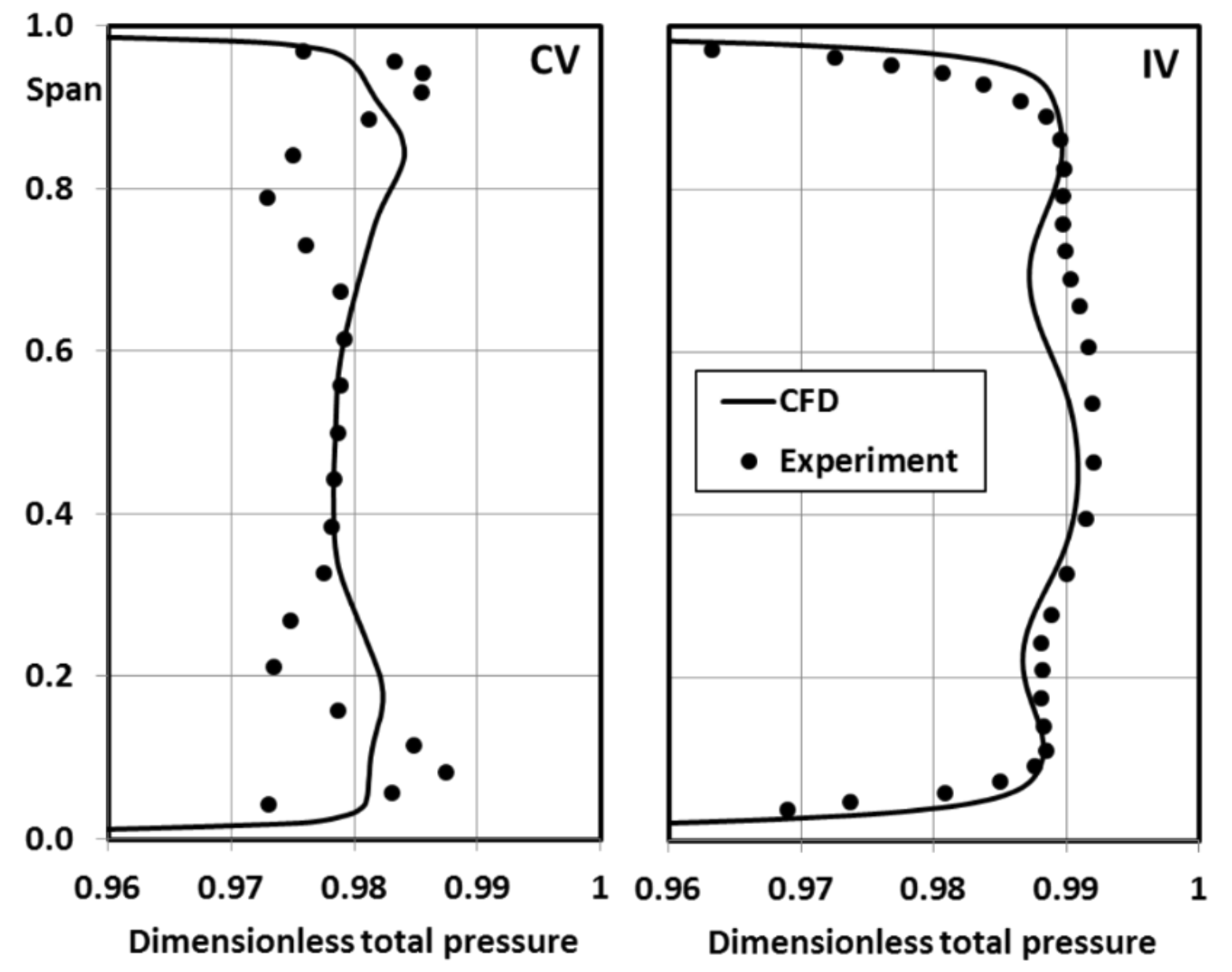

Figure 5-10: Comparison of pitchwise-averaged total pressure between traverse measurements and predictions downstream of conventional (left) and integrated (right) vanes

Figure 5-11 similarly compares pitchwise-averaged yaw downstream of the vane trailing edges between experiments and CFD. The overall agreement is found to be good, with only a slight shift in yaw (maximum of approximately $2^{0}$ ) across the span. The necessary turning, $71.4^{\circ}$ and $71.0^{\circ}$ for conventional and integrated vane design respectively, is maintained. The local turning is however considerably altered with increased turning towards the endwalls and reduced turning towards the midspan for the integrated vane. The effect of the altered yaw angle distribution on the rotor's aerodynamic performance has to be investigated in more detail with full stage simulations. More homogeneous turning could potentially be achieved by introducing different levels of vane compound lean. 

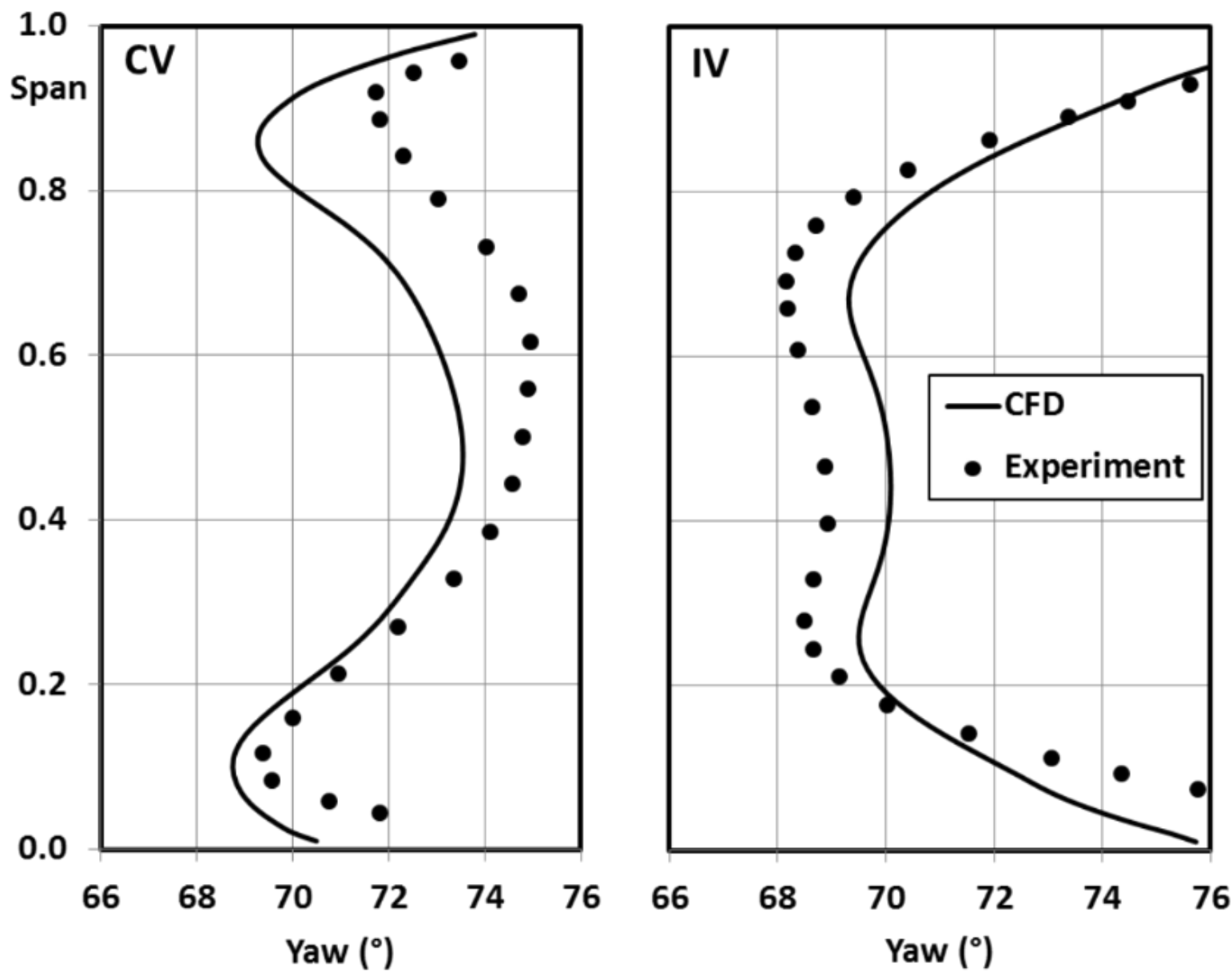

Figure 5-11: Comparison of pitchwise-averaged yaw between traverse measurements and predictions downstream of conventional (left) and integrated (right) vanes

\subsection{Static pressure measurements}

The integrated vane was equipped with static pressure tappings along the chord in order to obtain a better understanding of the flow around the vane, and to compare the loading distribution to the conventional vanes and to the numerical predictions. Experimental measurements were thus conducted for conventional and integrated vanes, with the turbulence grid installed upstream of the combustor transition duct. The pressure tappings were connected to the DSA via Scanivalve tubing. Since the DSA only features 16 channels (of which 6 are used to measure the pressures upstream and inside of the combustor transition duct), ten separate experiments had 
to be conducted to measure all 57 pressure tappings. The pressure stays very constant during the run, and averaging over the run's duration gives a static pressure value for each tapping. Using the total pressure inside of the combustor transition duct allows obtaining the isentropic Mach number for each location using the equation:

$$
M_{i s}=\sqrt{\left(\left(\frac{p_{0}}{p}\right)^{\frac{\gamma-1}{\gamma}}-1\right) \cdot \frac{2}{\gamma-1}}
$$

The same principle was used to acquire the isentropic Mach numbers of the conventional vanes. Figure 5-12 shows an experimental comparison, complimented by numerical values, of the isentropic Mach number plotted against the integrated vane's axial chord at midspan between conventional and integrated vanes.

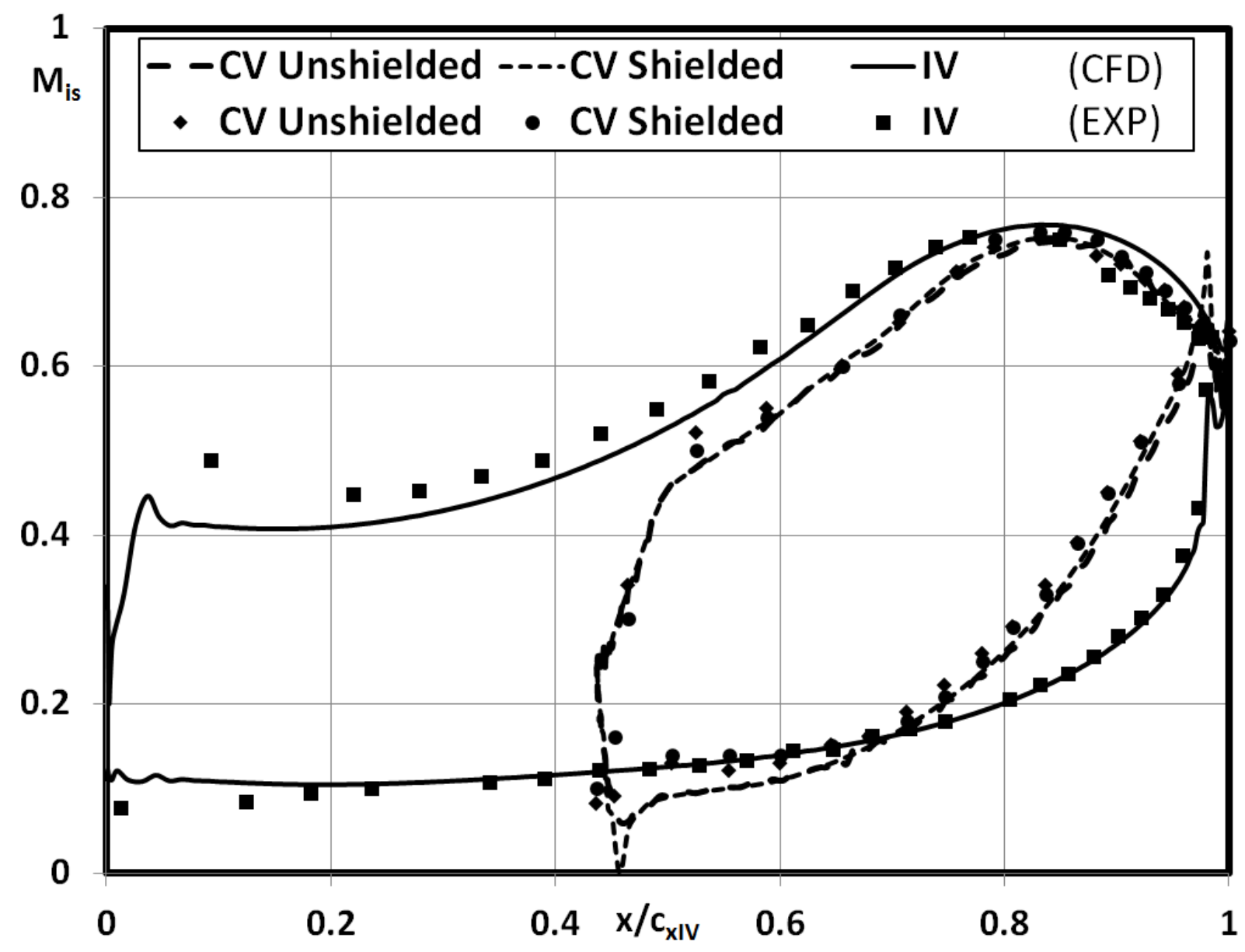

Figure 5-12: Isentropic Mach number surface distribution for conventional and integrated vanes at midspan; lines and dots represent numerical and experimental results respectively 
CFD and experiments show good agreement for both cases. The Mach numbers along shielded and unshielded conventional vanes are nearly identical. The acceleration on the integrated vane's suction surface is smoother than for the conventional vanes and peak Mach numbers, achieved at the same axial position of $c_{x \mid V}=0.84$, are nearly identical in magnitude for integrated and conventional vanes. The loading of the integrated vane is nearly equal to the conventional vanes' combined loading and is distributed along the integrated vane's increased chord length.

\subsection{Aerodynamic losses}

Figure 5-12 showed that the axial distance of high Mach numbers and of boundary layer diffusion on the suction surface is similar for the conventional and integrated vanes. Since these occur on only one integrated vane instead of two conventional vanes, the affected surface area is significantly reduced. The reduction of the diffusion region and of the area of high velocities is illustrated in Figure 5-13 and results in a reduction of profile loss. 

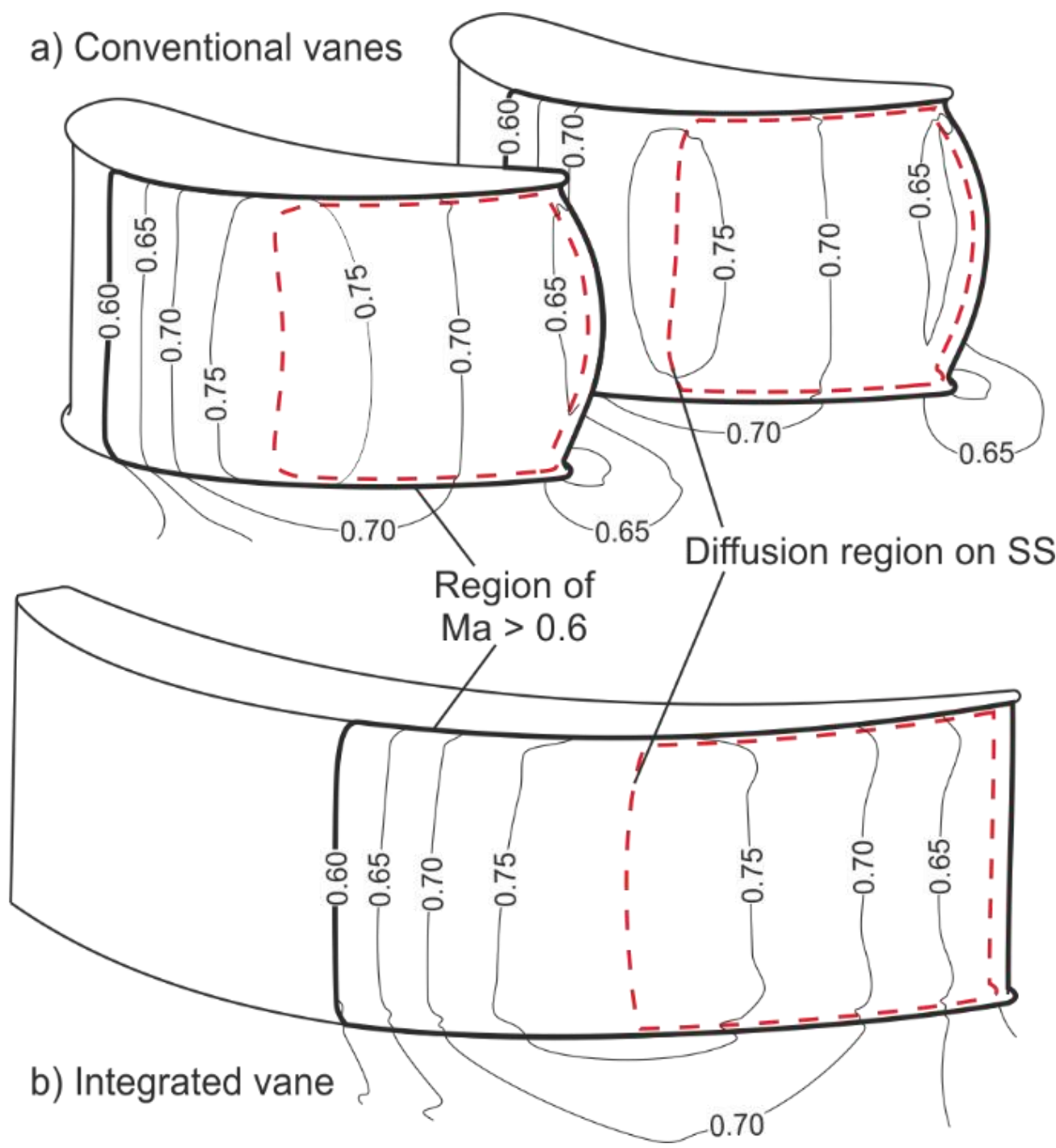

Figure 5-13: Schematic regions of high isentropic Mach numbers and of boundary layer diffusion on suction surfaces

Figure 5-14 shows contours of entropy generation rate per unit surface area on the hub endwall for conventional and integrated vanes. The values are nondimensionalised by $\rho_{2} v_{2}{ }^{3} / T_{2}$. The horseshoe-vortex system, induced by the interaction of upstream boundary layer and the unshielded conventional vane leading edge, and resulting passage vortex are apparent (indicated by dashed white line). It is likely (although not conclusively established in literature) that the thin boundary layer developing downstream of this passage vortex becomes turbulent very quickly due to the high levels of freestream turbulence from the combustor. The resulting thin boundary layer gives rise to a region of high entropy generation. 


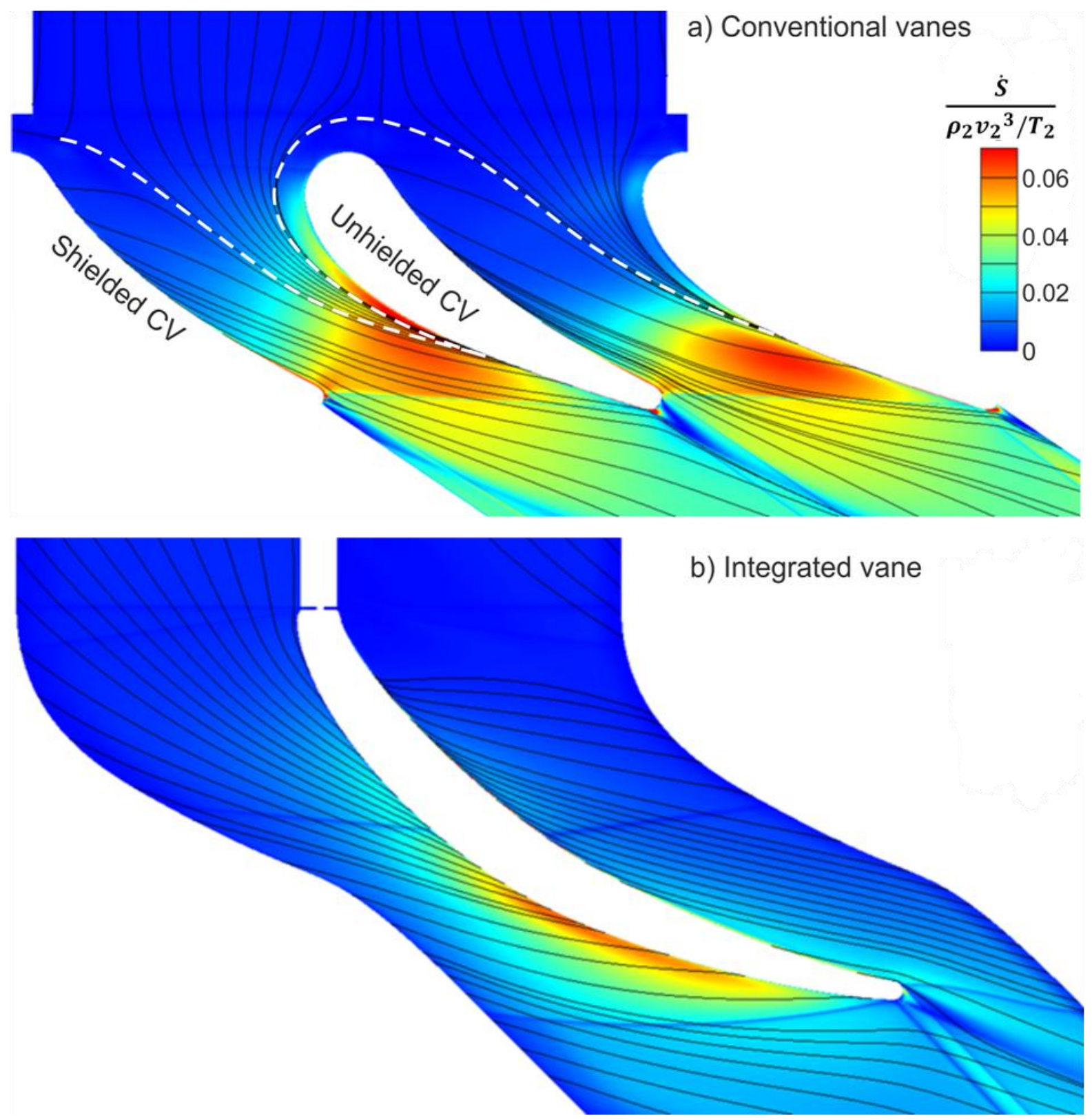

Figure 5-14: Dimensionless entropy generation rate on hub endwall of conventional (a) and integrated (b) vane domain; surface streamtraces

This phenomenon is absent for the integrated vane design due to the lack of a leading edge stagnation pressure region. Thus, the integrated vane design removes the horseshoe-vortex system, allowing the thick, uniformly distributed inlet boundary layer to be transported through the passage. This results in an overall thicker endwall boundary layer inside the passage compared to the conventional vane design (also seen in Figure 5-8) and considerably reduced entropy generation rate. This can be seen schematically in Figure 5-15. 
a) Conventional vanes

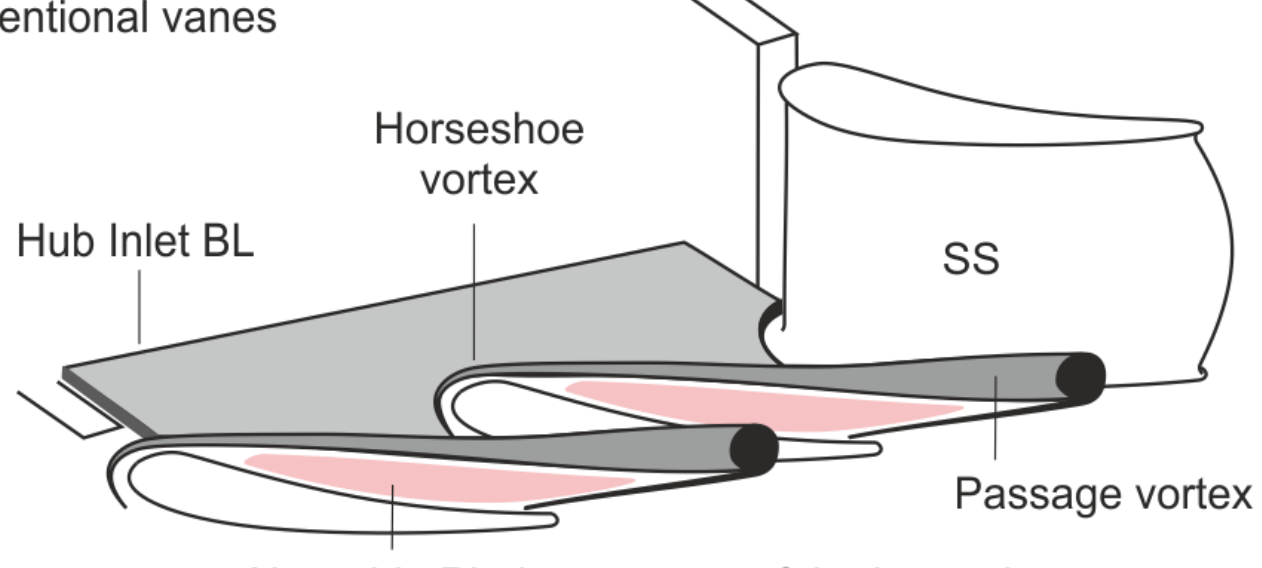

New, thin $B L$ downstream of the horseshoe vortex

b) Integrated vane

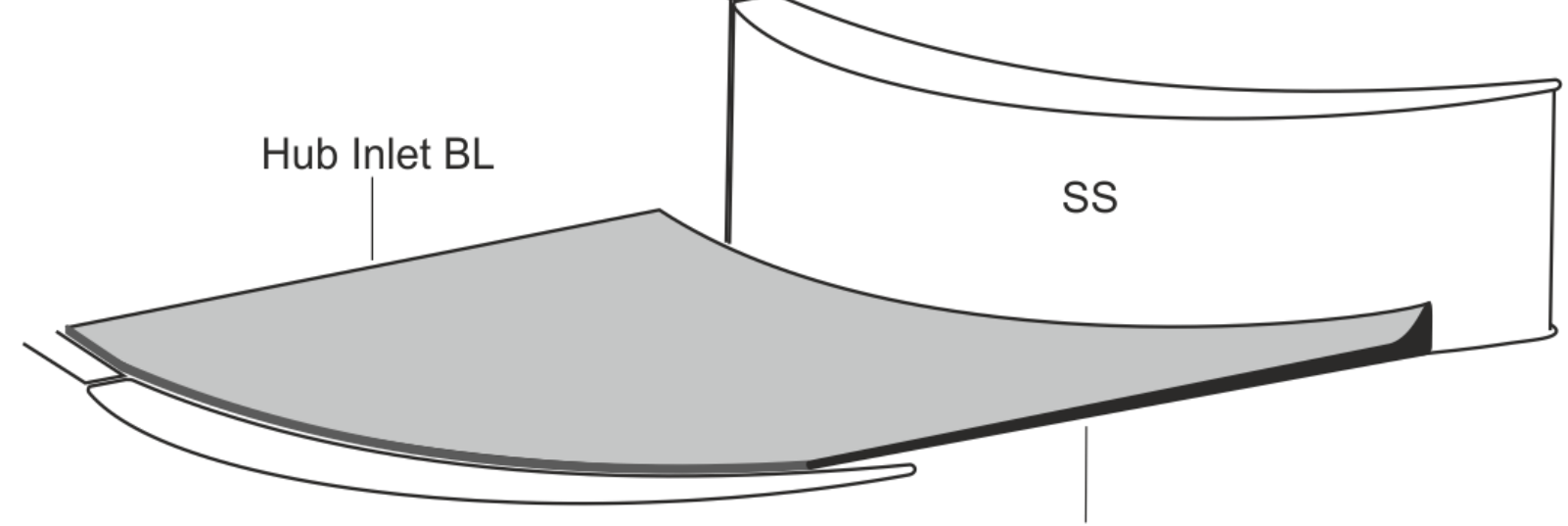

Thickening \& cross-passage migration of low momentum BL fluid

Figure 5-15: Schematic of endwall boundary layer development for conventional (a) and integrated (b) vane design

In order to sum up and compare the losses incurred by conventional and integrated vanes, the total pressure loss coefficient will be used. This coefficient gives an indication of the loss generated in a turbine blade (Denton J. , 1993) and was defined in equation (3). Figure 5-16 shows the massflow-weight averaged total pressure loss coefficient through both domains. Since the integrated vane is shifted axially into the transition duct, loss generation starts earlier (i.e. at $x / \mathrm{c}_{x \mid \mathrm{V}}=0$ ). This is, however, offset by the illustrated reduction in profile as well as endwall loss for the integrated vane and results in a considerable reduction of total pressure loss coefficient at the exit of the domain $\left(27 \%\right.$ at $\left.x / c_{x \mid V}=1.5\right)$. A further contributor to this reduction is the halving 
of the number of trailing edges (while integrated and conventional vane trailing edge thicknesses are nearly identical). Full stage simulations will need to examine if the altered flowfield leads to an increased loss in the rotor and how the stage efficiency is affected.

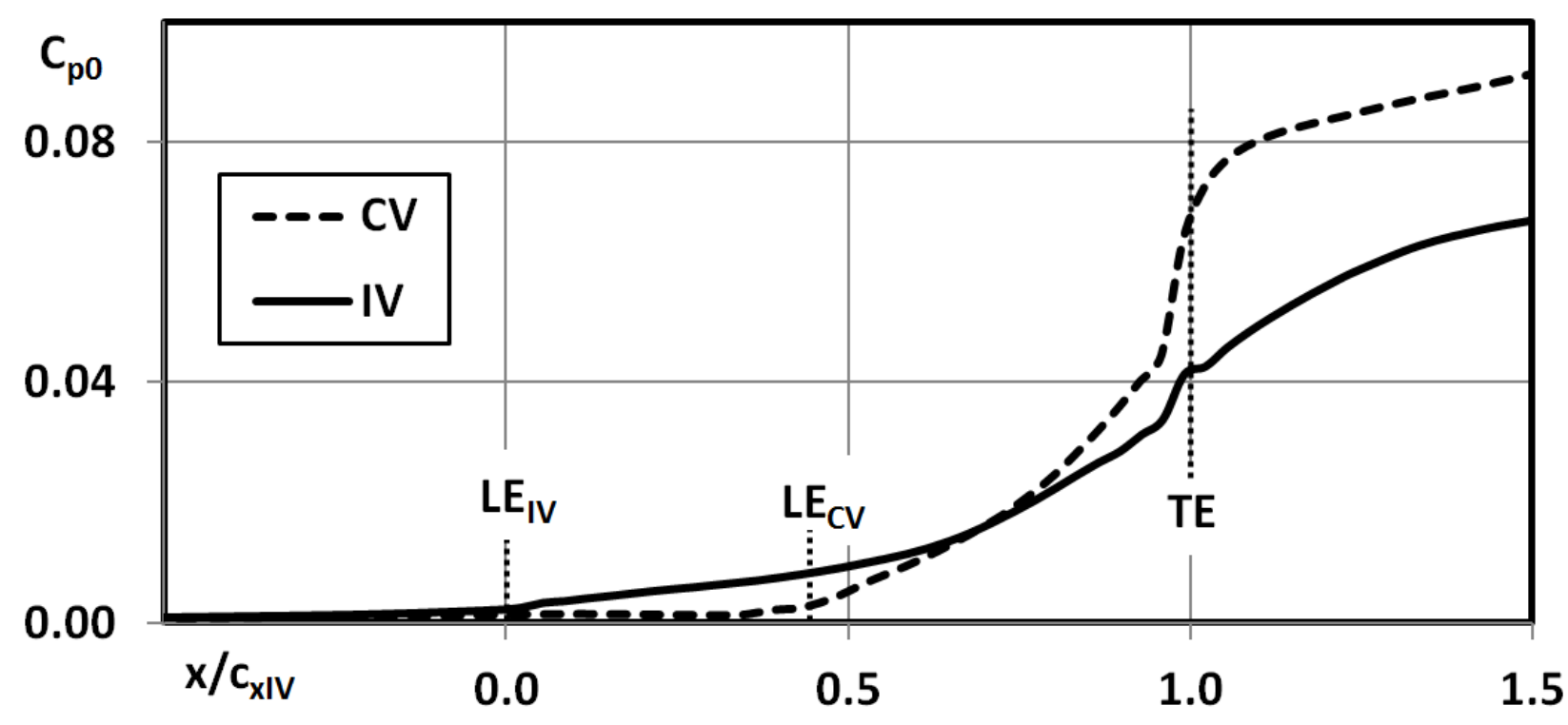

Figure 5-16: Growth of total pressure loss coefficient through linear CFD domains 


\section{Chapter 6: Thermal Performance of Integrated Vane}

\subsection{Abstract}

This chapter focuses on a detailed comparison of the thermal performance of the integrated vane design in comparison to the conventional vane design. A significant reduction in heat transfer levels for the integrated vane surfaces (pressure and suction side) is shown and attributed to the transferral of the combustor wall boundary layer onto the integrated vane. Similar levels of heat transfer coefficient reductions are observed on the integrated vane endwall, caused by a removal of the horse-shoe vortex system and a resulting thicker endwall boundary layer. The experimental measurements are supported and validated by numerical simulations. Finally, the reductions in the heat transfer coefficient levels are also validated by using Reynold's empirical correlation of the heat transfer in a turbulent boundary layer for a flat plate. An overview of the results presented in this chapter is given by Jacobi and Rosic (2016).

\subsection{Experimental comparison of vane surface heat transfer}

Experimental measurements using the infrared camera were conducted for the conventional vanes and integrated vane to obtain the heat transfer coefficient on vanes and endwalls. A turbulence grid was installed upstream of the combustor transition duct to achieve engine representative turbulence levels of approximately $12 \%$. The subsequent measurements will present the heat transfer characteristics on the vanes suction surfaces, pressure surfaces and leading edges. 
Figure 6-1 shows the heat transfer coefficient on the conventional and integrated vanes suction surfaces and the measured area is indicated by the grey surfaces in the schematic.
a) Conventional vanes
b) Integrated vane
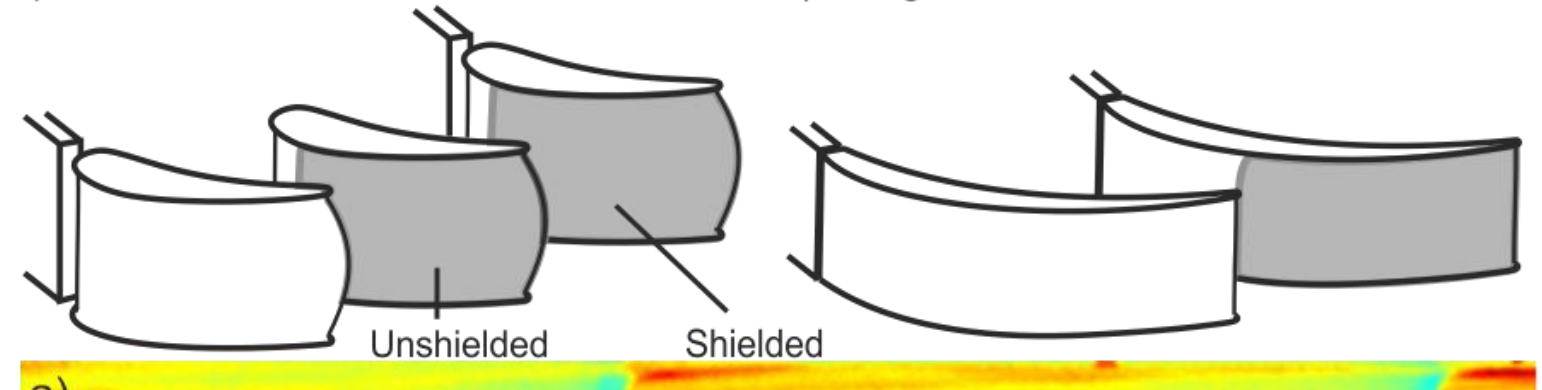

a)

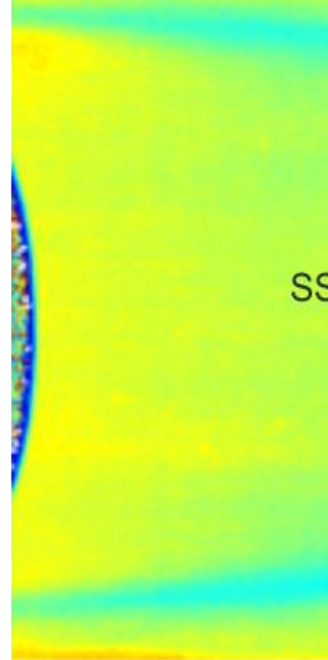

SS

TE

SS

TE

b)

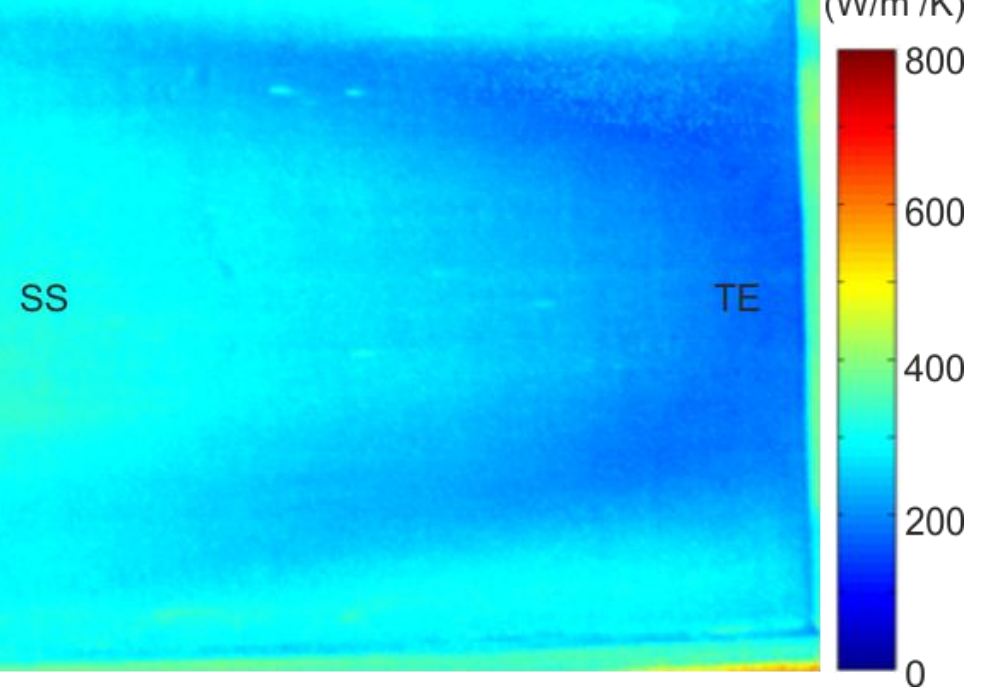

Figure 6-1: Measured heat transfer coefficient on conventional vane (a) and integrated vane (b) suction surfaces 
It can be seen that the levels of the heat transfer coefficient are qualitatively similar for both conventional vanes. Heat transfer coefficient levels are slightly higher for the shielded vane compared to the unshielded vane, especially in regions close to the leading edge. This is caused by the upstream transition duct wall that generates additional turbulence. Moreover, the secondary flows on the conventional and integrated vanes suction surfaces can clearly be seen by the separation lines and reduced heat transfer coefficient values. The strength of the secondary flows on the integrated vane's suction surface is slightly increased in comparison to the conventional vanes (see increased spanwise position of the separation lines) due to the increased loading and reduced aspect ratio. This trend agrees well with the aerodynamic traverse measurements presented in Figure 5-8. Overall, a significant reduction in the magnitude of the heat transfer coefficient is evident, for peak values as well as overall. Peak values (obtained in the region of peak velocities) are reduced by more than $40 \%\left(600 \mathrm{~W} /\left(\mathrm{m}^{2} \mathrm{~K}\right)\right.$ to $\left.360 \mathrm{~W} /\left(\mathrm{m}^{2} \mathrm{~K}\right)\right)$ and minimum values at the trailing edge are reduced by approximately $60 \%\left(450 \mathrm{~W} /\left(\mathrm{m}^{2} \mathrm{~K}\right)\right.$ to $\left.180 \mathrm{~W} /\left(\mathrm{m}^{2} \mathrm{~K}\right)\right)$.

Figure 6-2 shows the distribution of the heat transfer coefficient on the shielded conventional and the integrated vane's pressure surface. It can be seen that the stagnation pressure region at the leading edge of the shielded conventional vane leads to high heat transfer coefficient values of approximately $400 \mathrm{~W} /\left(\mathrm{m}^{2} \mathrm{~K}\right)$. The levels of the heat transfer coefficient also quickly increase on the pressure surface in downstream direction due to the acceleration of the flow. In comparison, levels of heat transfer on the integrated vane are very homogenous and only slightly increase on the pressure surface in downstream direction due to the very slow acceleration of the flow in this region (the flow only starts to significantly increase after approximately $80 \%$ of axial chord, as seen in Figure 5-12). 


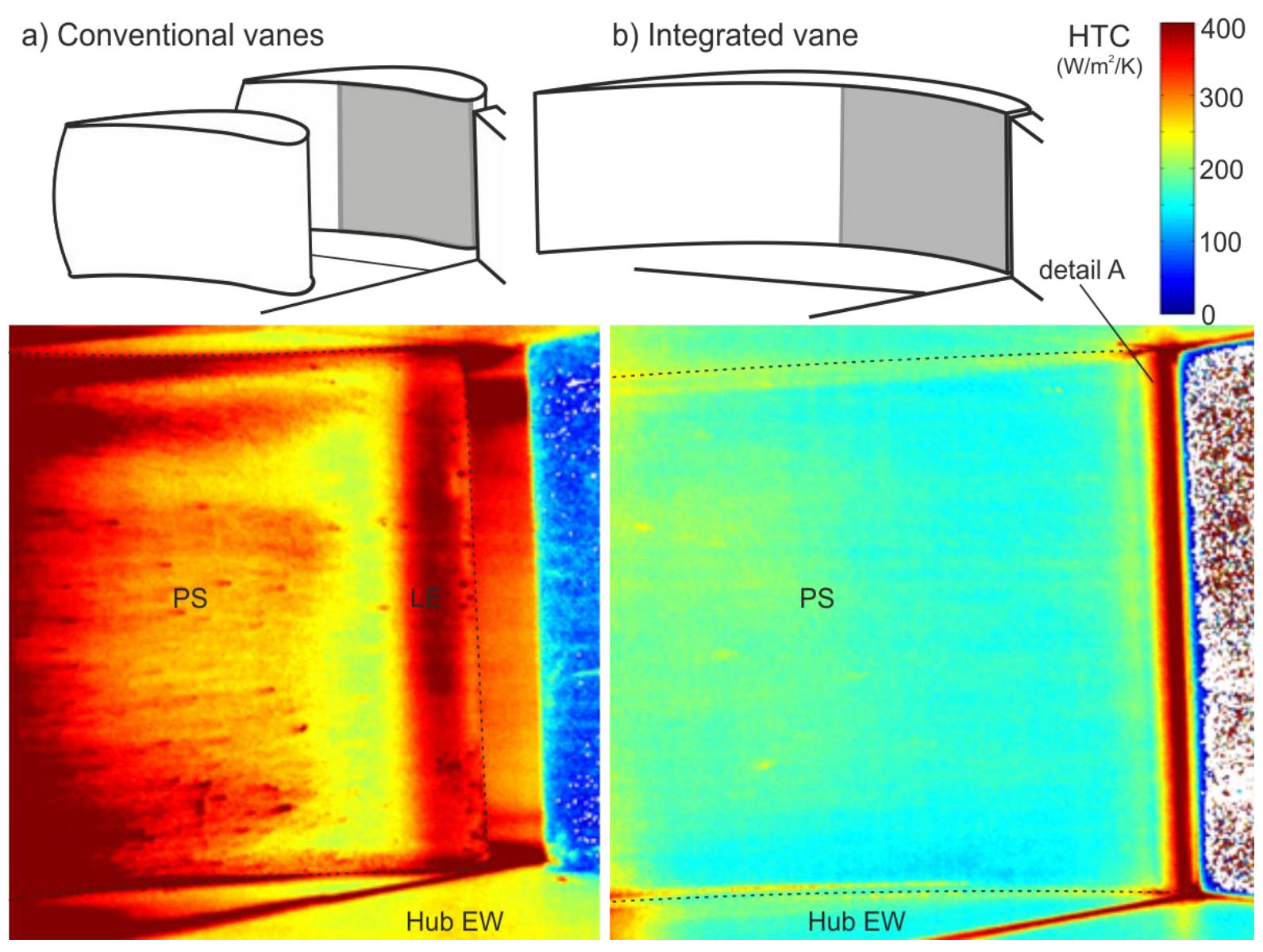

Figure 6-2: Measured heat transfer coefficient on shielded conventional vane's (a) and integrated vane's (b) pressure surface

The thin region of high heat transfer coefficient values at the leading edge of the integrated vane (see detail $\mathrm{A}$ ) is due to the high curvature and a slight misalignment with the combustor wall. Excluding this thin region results in a reduction in the heat transfer coefficient on the integrated vane's pressure surface by approximately $40 \%$ $50 \%$.

Figure 6-3 shows the heat transfer coefficient distributions on the conventional and integrated vanes leading edges. 
a) Conventional vane
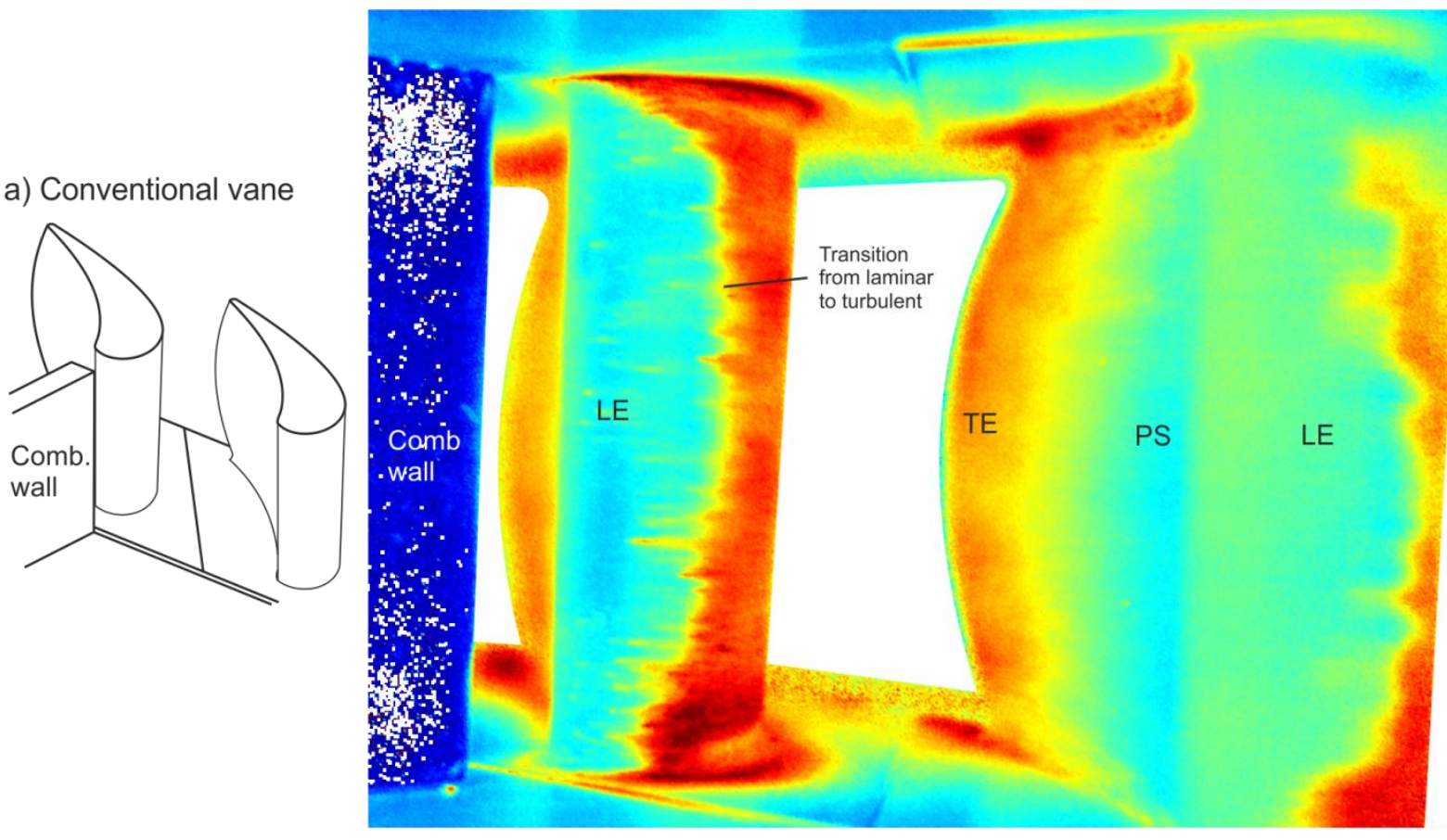

b) Integrated vane
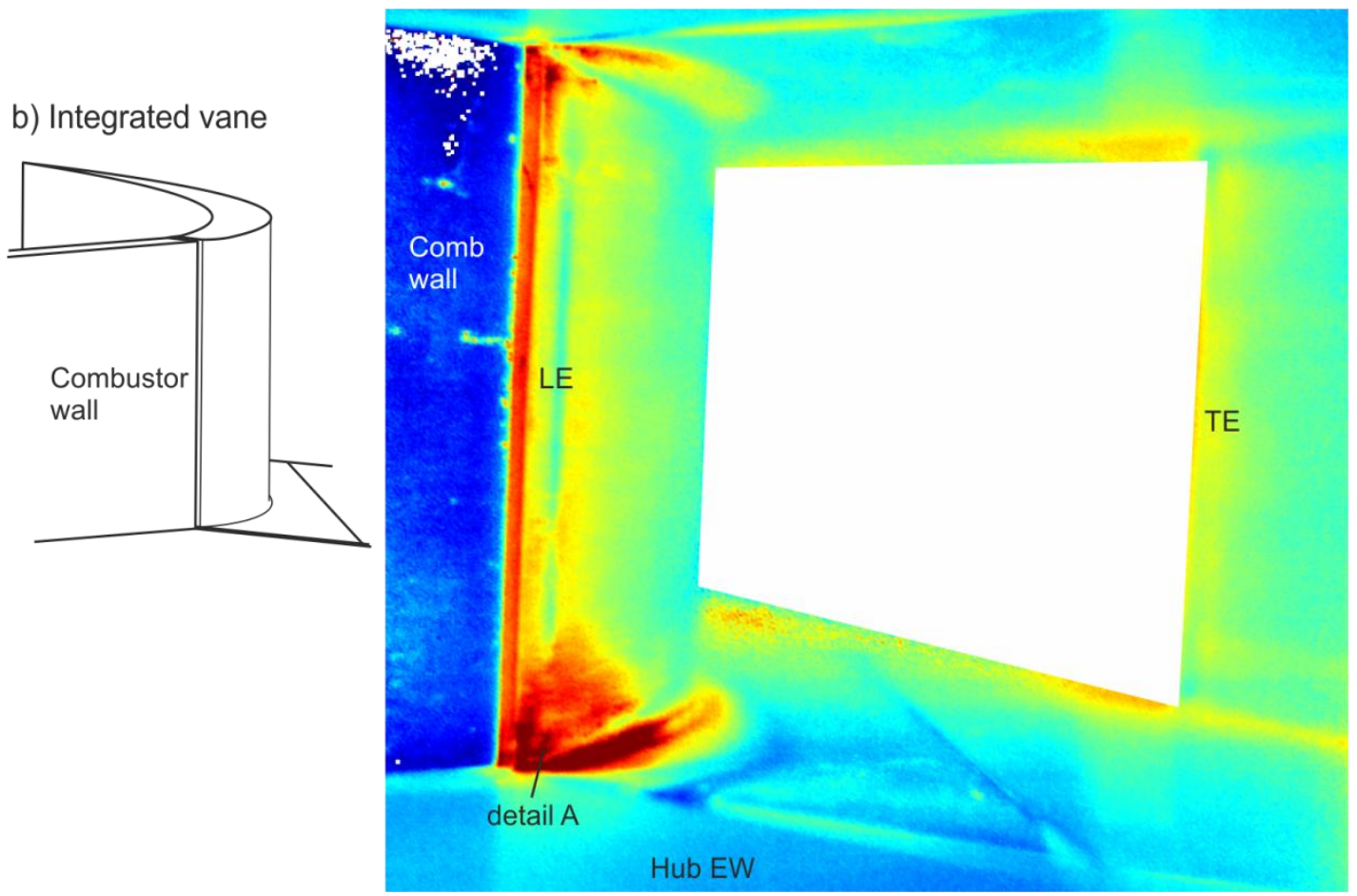

HTC

$\left(\mathrm{W} / \mathrm{m}^{2} / \mathrm{K}\right)$

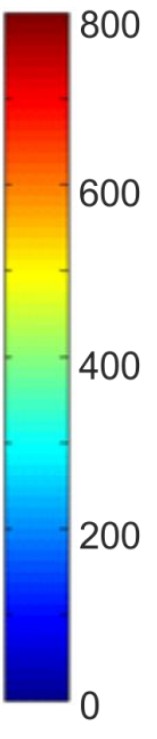

Figure 6-3: Measured heat transfer coefficient on the conventional (a) and integrated (b) vanes leading edges

It can be seen in Figure 6-3a that HTC levels are slightly lower on the shielded (300 $\left.\mathrm{W} /\left(\mathrm{m}^{2} \mathrm{~K}\right)\right)$ than unshielded $\left(400 \mathrm{~W} /\left(\mathrm{m}^{2} \mathrm{~K}\right)\right)$ conventional vane. This is due to the shielding of the upstream combustor wall (i.e. lower leading edge stagnation pressure of shielded vane leads to lower HTC levels). HTC levels rapidly increase 
towards the suction surface at the transition point from laminar to turbulent, with values up to $750 \mathrm{~W} /\left(\mathrm{m}^{2} \mathrm{~K}\right)$. HTC levels of $600 \mathrm{~W} /\left(\mathrm{m}^{2} \mathrm{~K}\right)$ are obtained at the trailing edge of shielded and unshielded vane. Heat transfer coefficient levels of the integrated vane, as seen in Figure 6-3b, are considerably lower. No transition line comparable to the conventional vane is evident. Neglecting the high HTC levels on the leading edge towards the hub in detail A (which are attributed to a slight protrusion of the integrated vane's leading edge with respect to the combustor wall and which should realistically be similar to the levels towards the casing) shows that peak HTC levels of $450 \mathrm{~W} /\left(\mathrm{m}^{2} \mathrm{~K}\right)$ are obtained on the leading edge and decrease from there onwards on the suction surface. Peak HTC values of $450 \mathrm{~W} /\left(\mathrm{m}^{2} \mathrm{~K}\right)$ are obtained on the pressure surface's trailing edge. This implies a reduction in HTC of $40 \%((450-750) / 750)$ at the leading edge and of $25 \%((450-600) / 600)$ at the trailing edge for the integrated vane.

To summarize, it has been shown that HTC levels are considerably reduced on the suction surface, pressure surface and leading edge for the integrated vane. A look onto the conventional and integrated vanes endwalls in Figure 6-3 already indicates a significant reduction on the endwalls as well, and the subsequent subchapter will investigate this in more detail.

\subsection{Experimental comparison of vane endwall heat transfer}

To obtain a full picture of the thermal characteristics of the integrated vanes in respect to the conventional vanes, one also has to take the endwalls into consideration. Figure 6-4 thus compares heat transfer coefficient distributions on the conventional and integrated vanes endwalls from an upstream view. 


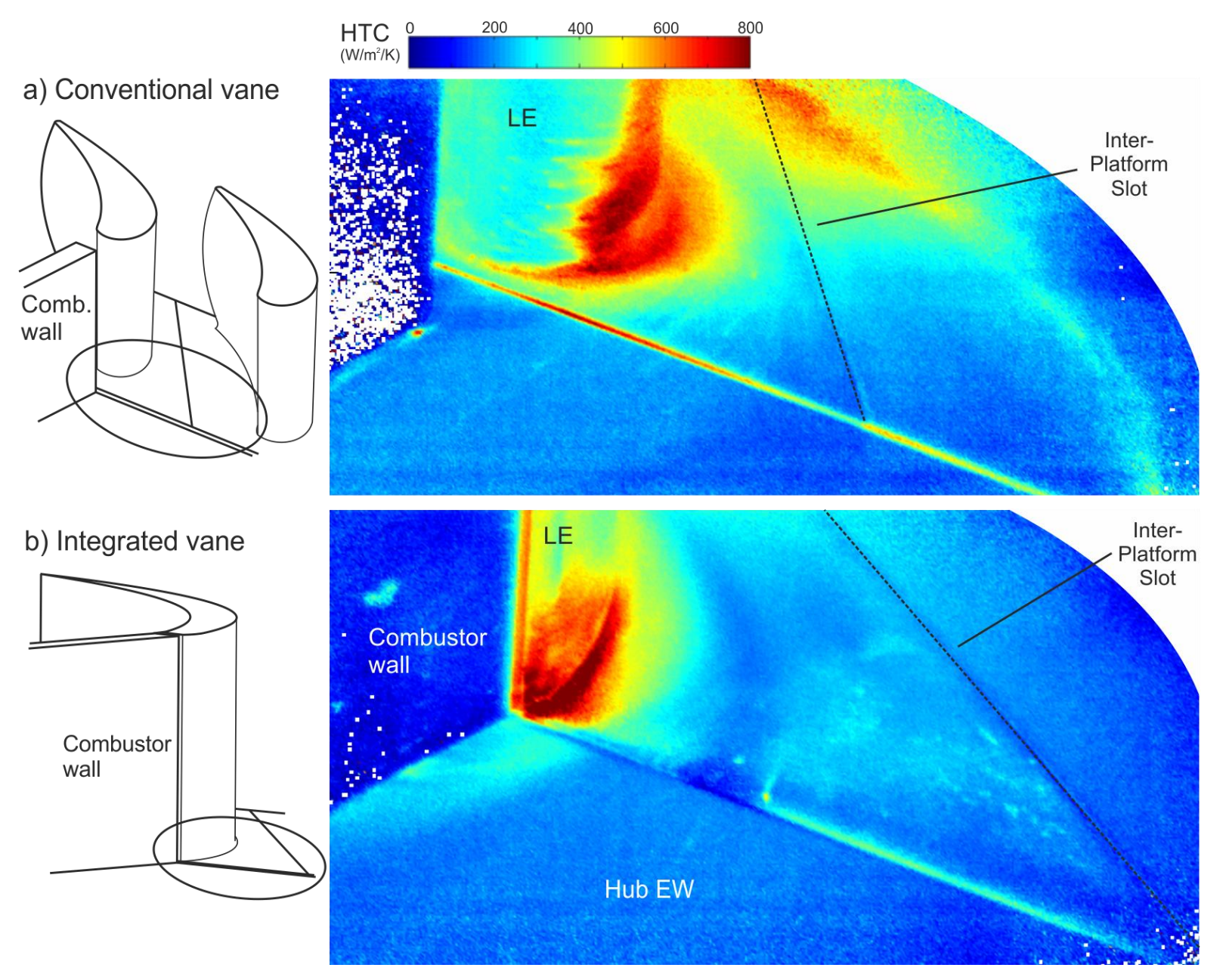

Figure 6-4: Measured heat transfer coefficient on the conventional (a) and integrated (b) vanes hub endwall (upstream view)

Figure 6-4a shows that the conventional vane HTC levels increase on the endwall along the passage from $200 \mathrm{~W} /\left(\mathrm{m}^{2} \mathrm{~K}\right)$ to $600 \mathrm{~W} /\left(\mathrm{m}^{2} \mathrm{~K}\right.$ ) (close to the unshielded vane pressure side) while the integrated vane HTC levels only slightly increase from 200 $\mathrm{W} /\left(\mathrm{m}^{2} \mathrm{~K}\right)$ to $300 \mathrm{~W} /\left(\mathrm{m}^{2} \mathrm{~K}\right)$ for the same distance. Qualitative and quantitative agreement to the HTC distributions on the leading edges of Figure 6-3 is very good.

Figure 6-5 similarly shows the heat transfer coefficient distribution of the conventional and integrated vanes hub endwalls from a downstream view. 

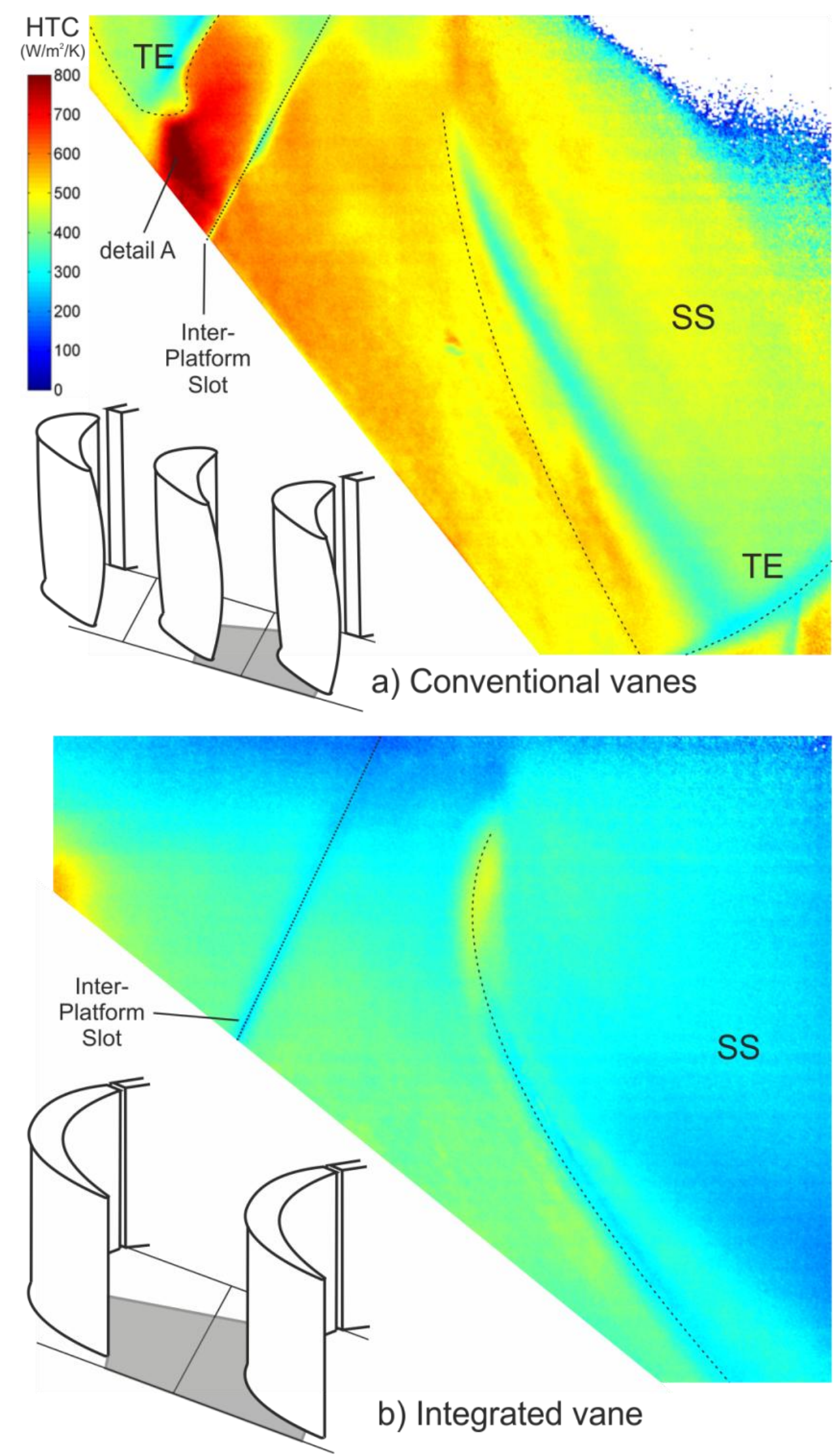

Figure 6-5: Measured heat transfer coefficient on conventional vane's (a) and integrated vane's (b) hub endwall 
Both plots partly show the heat transfer coefficient distributions on the vanes suction surfaces. These agree qualitatively and quantitatively very well to the previous measurements of Figure 6-1. The contour of the conventional vane's hub endwall shows high heat transfer coefficient values downstream of the passage vortex and downstream of the trailing edge (detail A, due to vortex shedding) of approximately $550-800 \mathrm{~W} /\left(\mathrm{m}^{2} \mathrm{~K}\right)$. The integrated vane on the other hand shows significantly lower values of the heat transfer coefficient of approximately $300-400 \mathrm{~W} /\left(\mathrm{m}^{2} \mathrm{~K}\right)$ (reduction of approximately $30 \%-50 \%$ ) and the distribution is much more homogeneous.

Understanding the flow physics of boundary layer development is key to comprehend this change in the endwall heat transfer characteristics. When the upstream endwall boundary layer approaches the conventional vane's leading edge stagnation pressure region, the low momentum fluid closer to the endwall is turned quicker than the fluid further from the endwall. This gives rise to a horse-shoe vortex system. It's pressure-side leg is convected to the adjacent vane's suction surface due to the passage pressure gradient, and interacts with the suction-side leg of the horse-shoe vortex. Downstream of this passage vortex, a very thin boundary layer is newly developed, giving rise to high heat transfer coefficient values. This is analogous to the explanation of the high entropy generation rate for the conventional vane design in Figure 5-14 and has already been shown in investigations by Graziani et al. (1980) for example. In contrast stands the integrated vane. In this case the upstream endwall boundary layer is not exposed to any vane and thus does not interact with a stagnation pressure region. The horse-shoe vortex system is thus removed and the boundary layer is simply transferred through the integrated vane passage. The thicker boundary layer then results in reduced levels of the heat transfer coefficient on the integrated vane's hub endwall. 
This can be clearly seen in the comparison of the boundary layer state for conventional and integrated vanes of Figure $6-6$ which shows an axial cut $(80 \%$ of $\mathrm{C}_{\mathrm{x} C v}$ downstream of the combustor wall trailing edge) of dimensionless total pressure $\overline{p_{0}}$ on the hub endwall.

a) Conventional vanes

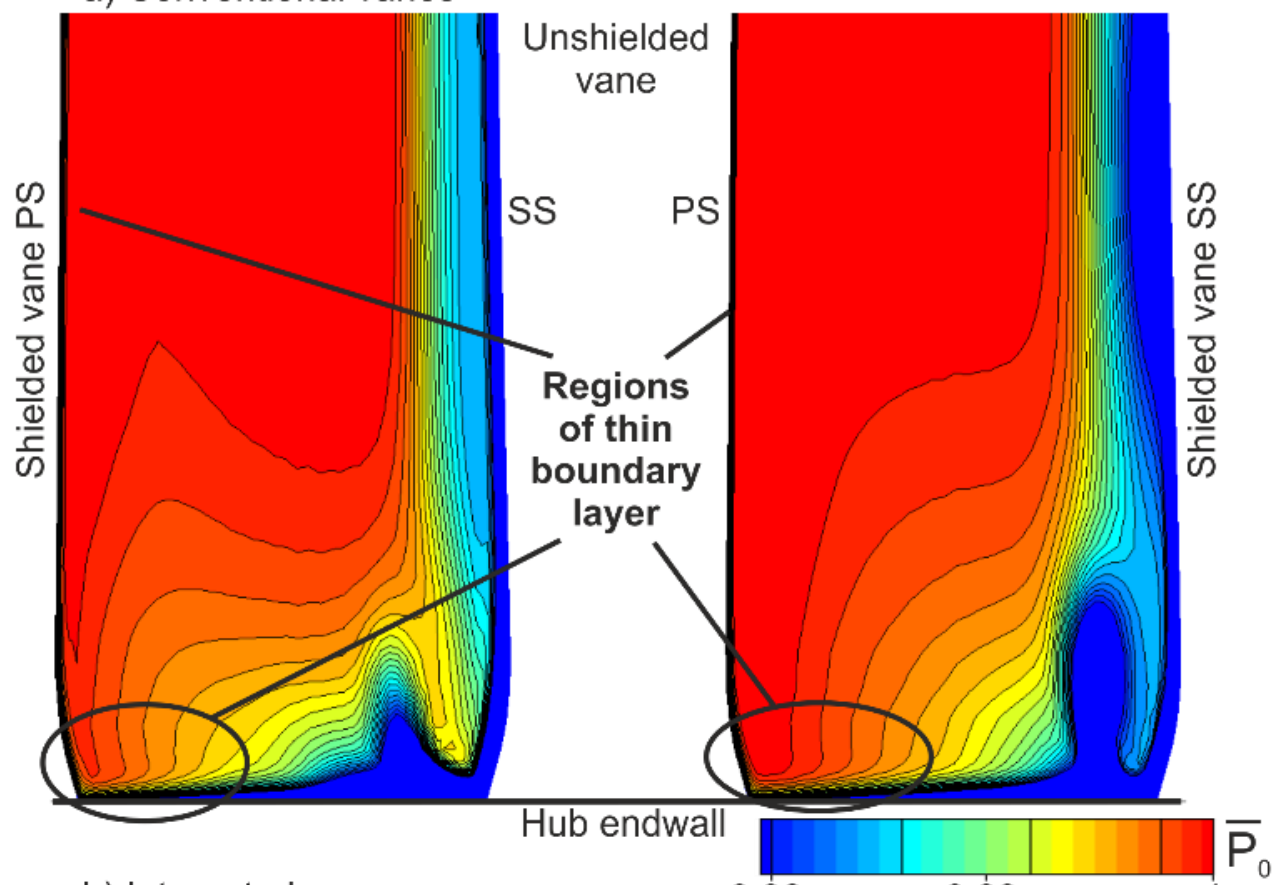

b) Integrated vane 0.98

$$
0.99
$$
1
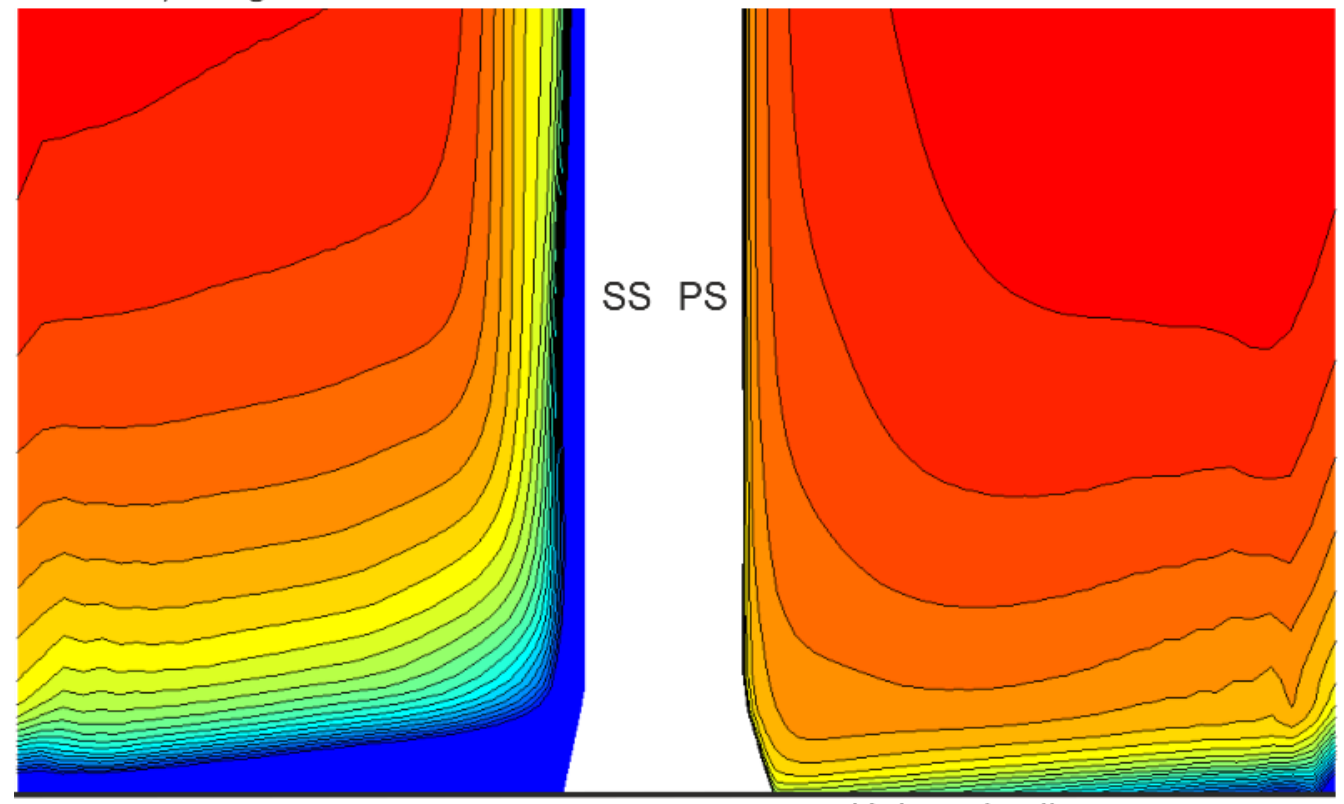

Hub endwall

Figure 6-6: Axial cut showing predicted dimensionless total pressure distribution for conventional vanes (a) and integrated vane (b) 
The distributions show a very thin boundary layer on the conventional vanes pressure surfaces and parts of its hub endwall. The integrated vane on the other hand exhibits considerably thicker boundary layers on pressure and suction surface and on the hub endwall. This results in the reduced heat transfer coefficient levels indicated in the prior measurements.

\subsection{Numerical comparison of vane heat transfer}

The alteration in the thermal characteristics illustrated by the experimental measurements is also validated by the numerical simulations. The heat transfer coefficient distributions predicted for the conventional and integrated vanes and endwalls are shown in Figure 6-7.
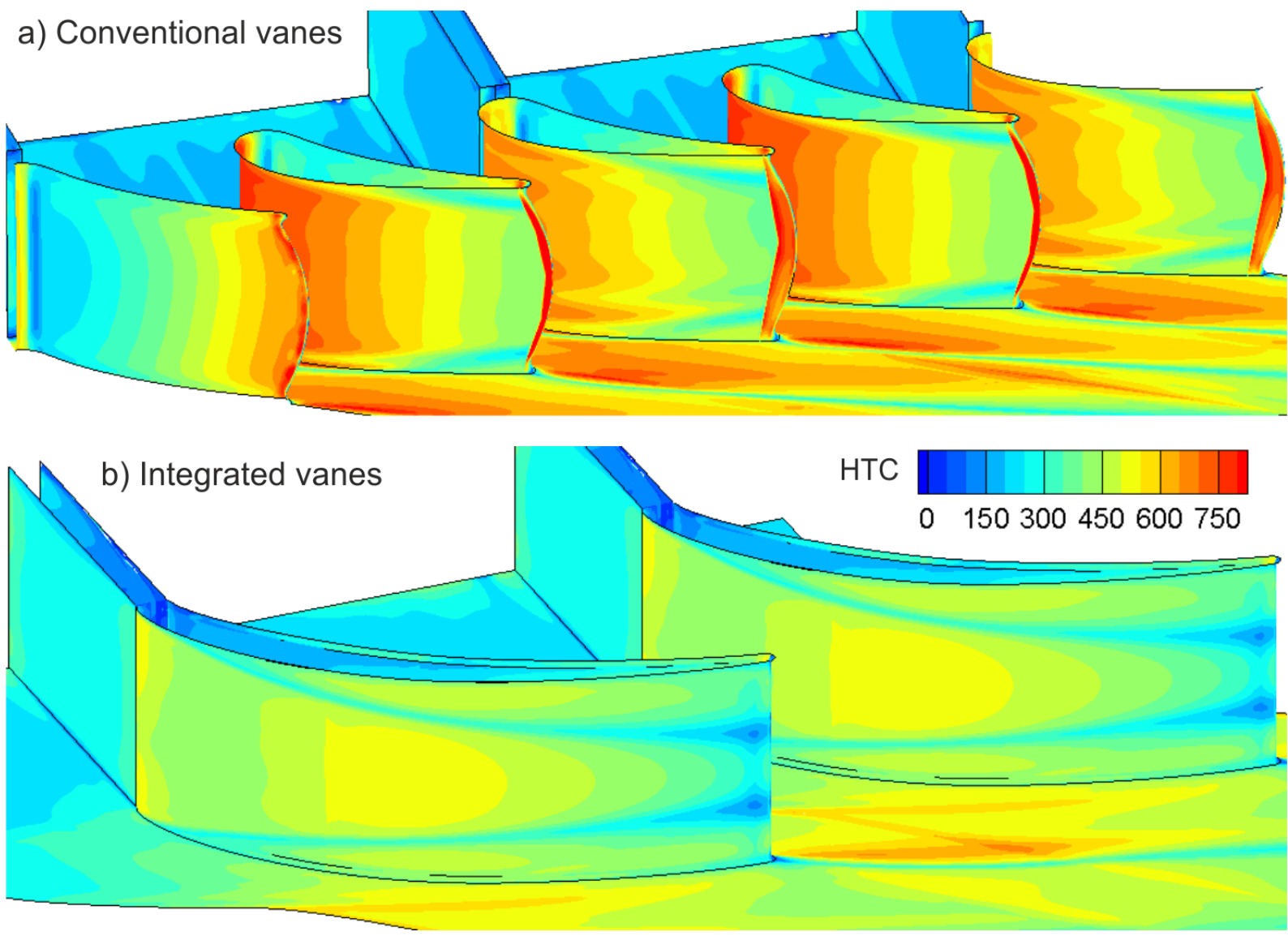

Figure 6-7: Predicted heat transfer coefficient distribution for conventional (a) and integrated (b) vane design 
Despite slightly over-predicting the magnitude of the heat transfer coefficient for both designs, the numerical results show qualitatively and quantitatively good agreement to the experimental measurements and confirm the illustrated trends: Overall significantly reduced levels of the heat transfer coefficient (by $40 \%-50 \%$ ) on the integrated vane due to the transferral of the combustor wall's boundary layer, and on the endwalls due to the removal of the horse-shoe vortex system.

A comparison of experimental HTC levels, complimented by numerical values, along the integrated vane's axial chord at midspan between two conventional vanes, shielded and unshielded, and the integrated vane is shown in Figure 6-8.

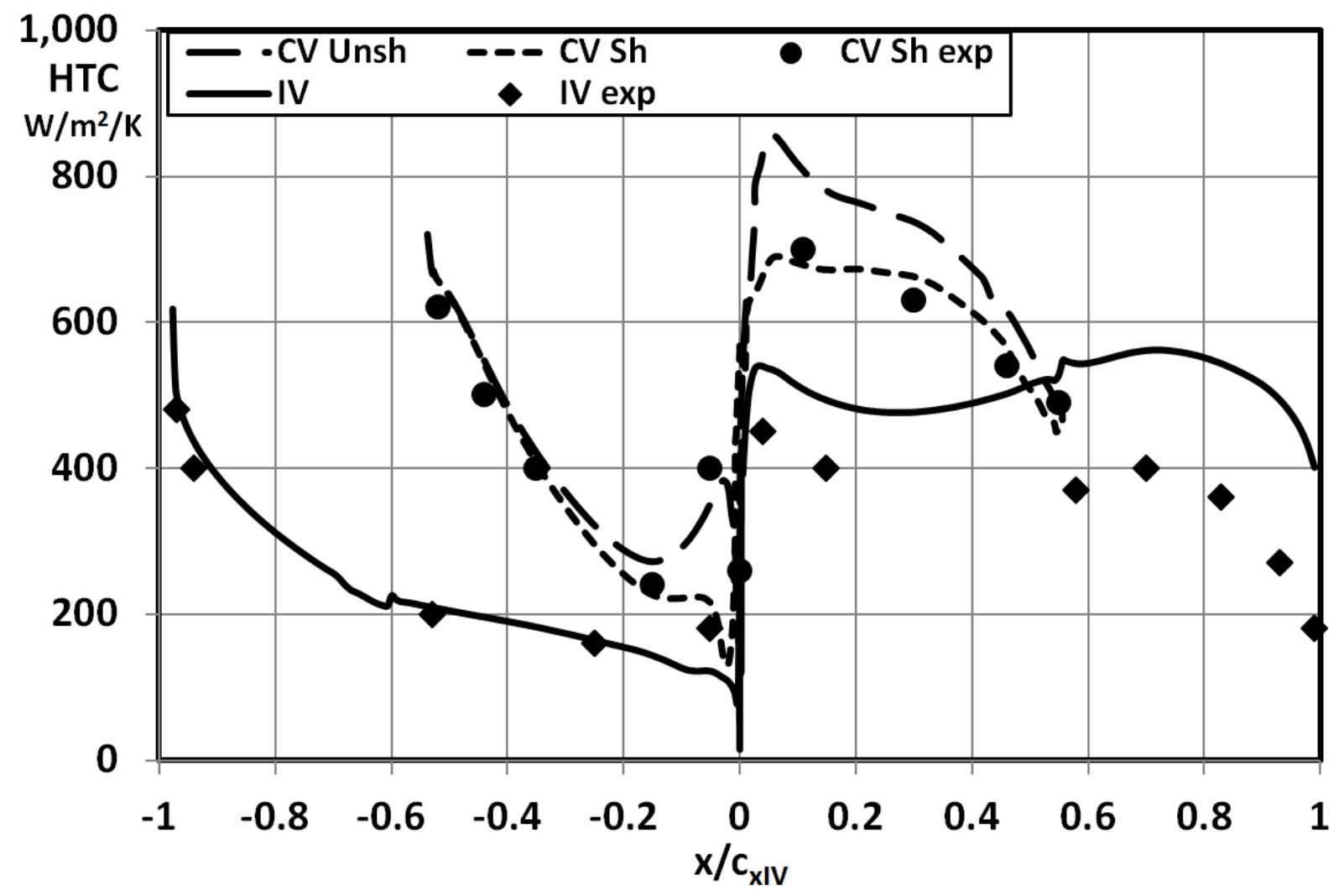

Figure 6-8: Heat transfer coefficient levels for conventional and integrated vanes at midspan; lines and dots represent numerical and experimental results respectively

The measured HTC-values were obtained through the three heat transfer coefficient plots of Figure 6-1, Figure 6-2 and Figure 6-3. The experimental HTC values show qualitatively and quantitatively good agreement to numerical results on the shielded conventional vane and on the integrated vane's pressure side. HTC levels on the 
integrated vane's suction side are overpredicted by the simulations but show similar qualitative trends. The CFD-simulations predict increased HTC values of the unshielded conventional vane compared to the shielded conventional vane, especially in the leading edge and suction surface region. The simulations do not incorporate a transition model, instead assuming fully turbulent flow. The experimental results of Figure 6-3 however clearly show the transition from laminar to turbulent on the conventional vanes leading edges. The lack of the transition in the simulations thus results in an overprediction of the unshielded vane's HTC levels.

The flowfield at the shielded conventional vane's leading edge is influenced by the upstream combustor wall. It sheds vortices and generates additional turbulence. A comparison between integrated and conventional vanes shows that HTC levels are considerably reduced overall by the integrated vane concept. The reduction of the heat transfer coefficient values on the integrated vane's pressure and suction surfaces in respect to the conventional vanes surfaces is caused mainly by the integrated vane's interaction and proximity with the upstream combustor wall. By physically minimising the distance between the integrated vane's leading edge and the combustor wall's trailing edge, while simultaneously converging their thickness, results in a removal of the stagnation pressure region conventionally found on a vane's leading edge and allows the boundary layer from the combustor wall to transfer onto the integrated vane. This, as previously described, leads to the considerable reduction in peak levels of heat transfer on the integrated vane (located on the suction side of the vanes' leading edge) by $37 \%$. This reduction could have a significant impact for gas turbines by allowing an increase in peak gas temperature or a reduction of cooling fraction, either way resulting in increased efficiency. 


\subsection{Empirical correlation of vane surface heat transfer}

The extent of the heat transfer coefficient reduction on pressure and suction surfaces can also be supported by Reynold's empirical correlation of the heat transfer in a turbulent boundary layer for a flat plate (Reynolds, Kays, \& Kline, 1958):

$$
S t \cdot\left(\frac{T_{W}}{T_{\infty}}\right)=0.0296 \cdot \operatorname{Pr}^{-0.4} \operatorname{Re}_{x}{ }^{-0.2}
$$

With $S t=\frac{N u}{R e \cdot P r}=\frac{h \cdot x}{k \cdot R e \cdot P r}$ this equation can be abbreviated to obtain $h \sim \frac{R e_{x}^{0.8}}{x} \sim x^{-0.2}$. Assuming an identical axial position of the conventional and integrated vane's trailing edge, taking into account the combustor wall for the integrated vane and neglecting it for the conventional vane, and disregarding the effect of streamwise curvature results in a reduction in the heat transfer coefficient for the integrated vane (on both pressure and suction surface) in the order of $60 \%-25 \%$ ranging from the conventional vane's leading to trailing edge. Figure 6-9 schematically shows the simplification of the conventional and integrated vanes, the formula for the ratio of their HTC levels and finally a graph of the HTC ratio on the pressure side over the conventional vane's chord. Also shown are the corresponding experimental HTC ratios at the leading edge, midchord and trailing edge, which all agree rather well with the empirical correlation prediction. 


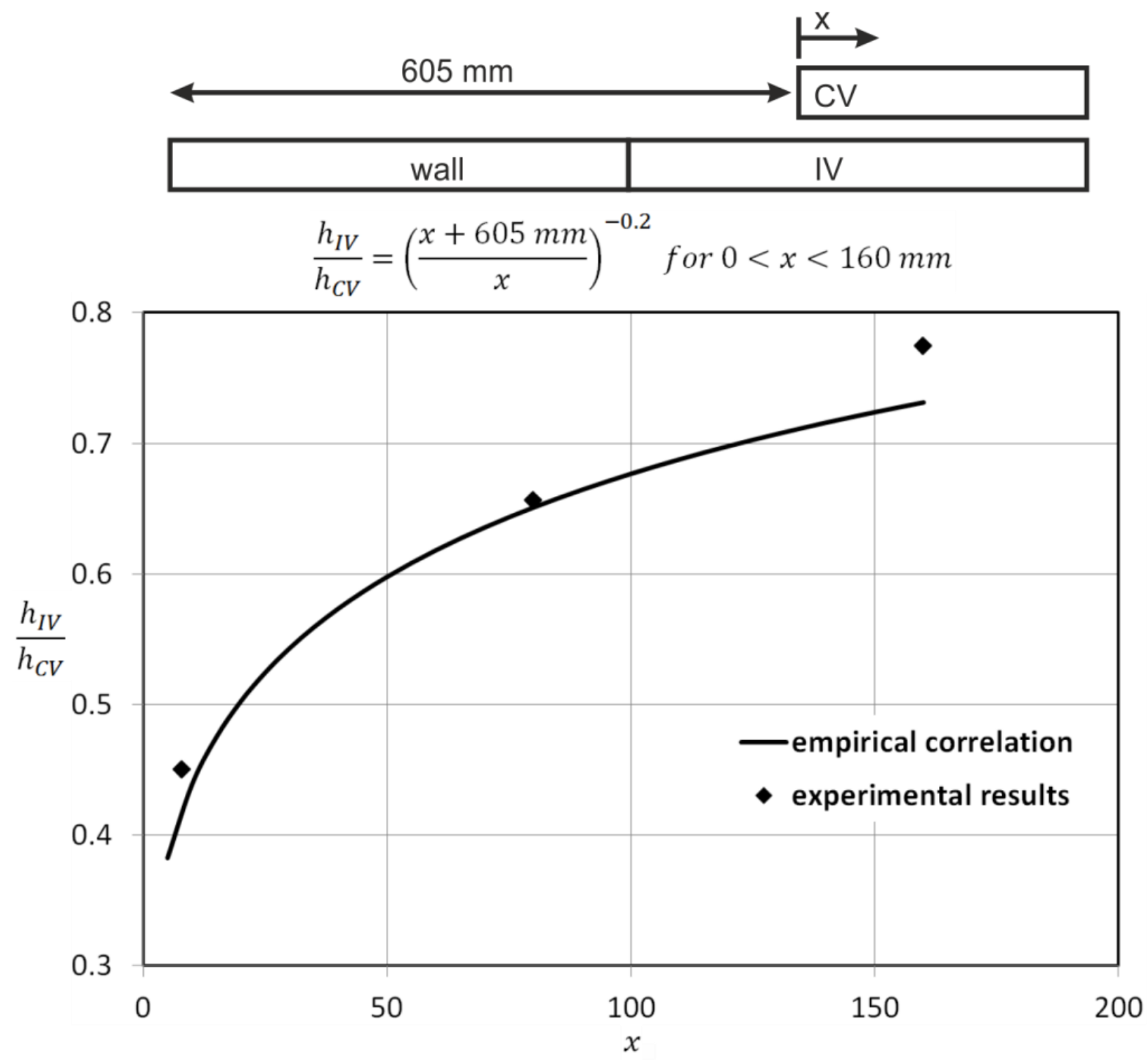

Figure 6-9: Empirical correlation for the ratio of HTC between conventional and integrated vane's pressure side along conventional vane's chord 


\section{Chapter 7: Full Stage Performance of Integrated Vane}

\subsection{Abstract}

This chapter focuses on the numerical investigation of the aerodynamic performance of the integrated combustor vane concept. First off, the transition from linear to annular domains is described. Subsequently it is shown that a considerable gain in stage efficiency is achieved by the integrated vanes. This is caused by a reduction in loss in the stator (which was previously shown in chapter 4) and an improved rotor performance. The latter is attributed to a halving of the number of stator wakes passing through the rotor, and a reduction in spanwise variation of rotor incidence angles. It is also described how the integrated vane design leads to an increased pitchwise variation of the flowfield downstream of the stator compared to the conventional vane design, and the effect thereof on the flowfield within and downstream of the rotor passage. The annular full stage simulations prove the validity of the concept for realistic gas turbine applications. An overview of the results presented in this chapter is given by Jacobi et al. (2017).

\subsection{Influence of transition from linear to annular domain}

Annular simulations were performed for the integrated vane in order to analyse the effect of radial momentum on the distributions downstream of the vane. The same inlet and outlet boundary conditions as for the linear simulations were initially applied to allow drawing a comparison with the prior linear simulations.

Figure 7-1a shows a comparison of the turning downstream of the integrated vanes between annular and linear setup. 


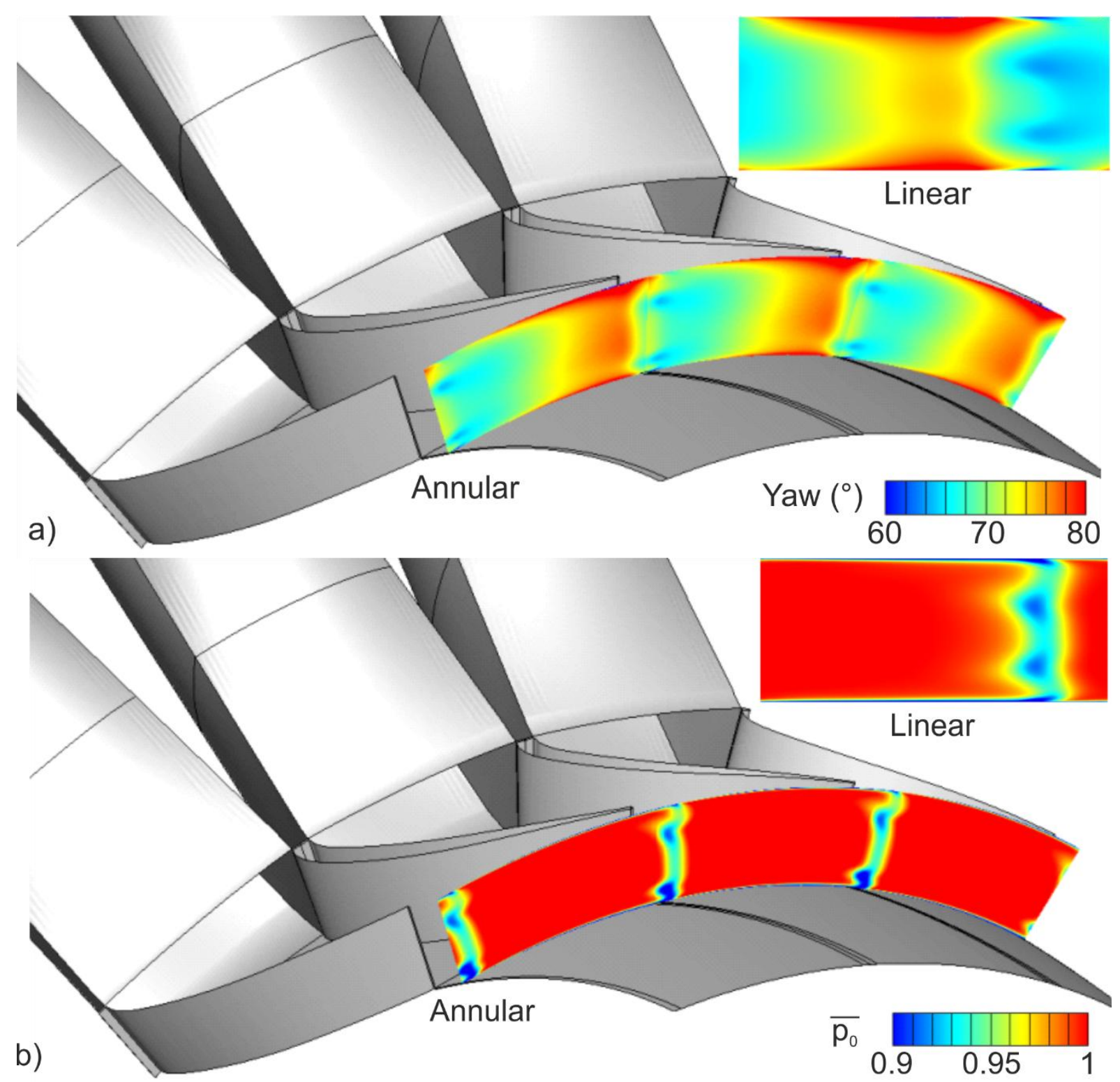

Figure 7-1: Predicted turning (a) and dimensionless total pressure (b) contours downstream of integrated vane for annular and linear domain

Nearly identical distributions are apparent with a slight reduction in overturning at the endwalls and slightly increased turning around midspan. Massflow-weighted averaging results in $73^{\circ}$ for integrated and $72^{\circ}$ for conventional vane design and verifies that turning is also maintained for the annular setup. Furthermore, a comparison of dimensionless total pressure between annular and linear setup is shown in Figure 7-1b. It can be seen that distributions remain similar. A slightly more pronounced hub loss core is evident and the boundary layer is thicker at the hub then at the casing. This is caused by the radial pressure gradient, with reduced static 
pressure at the hub and increased static pressure at the casing. Thus, CFD shows that the radial impact of the annular setup does not deteriorate the results and benefits exhibited by the linear CFD and experiments.

Figure 7-2 shows the surface distribution of HTC for the conventional and integrated vanes in the annular configuration.
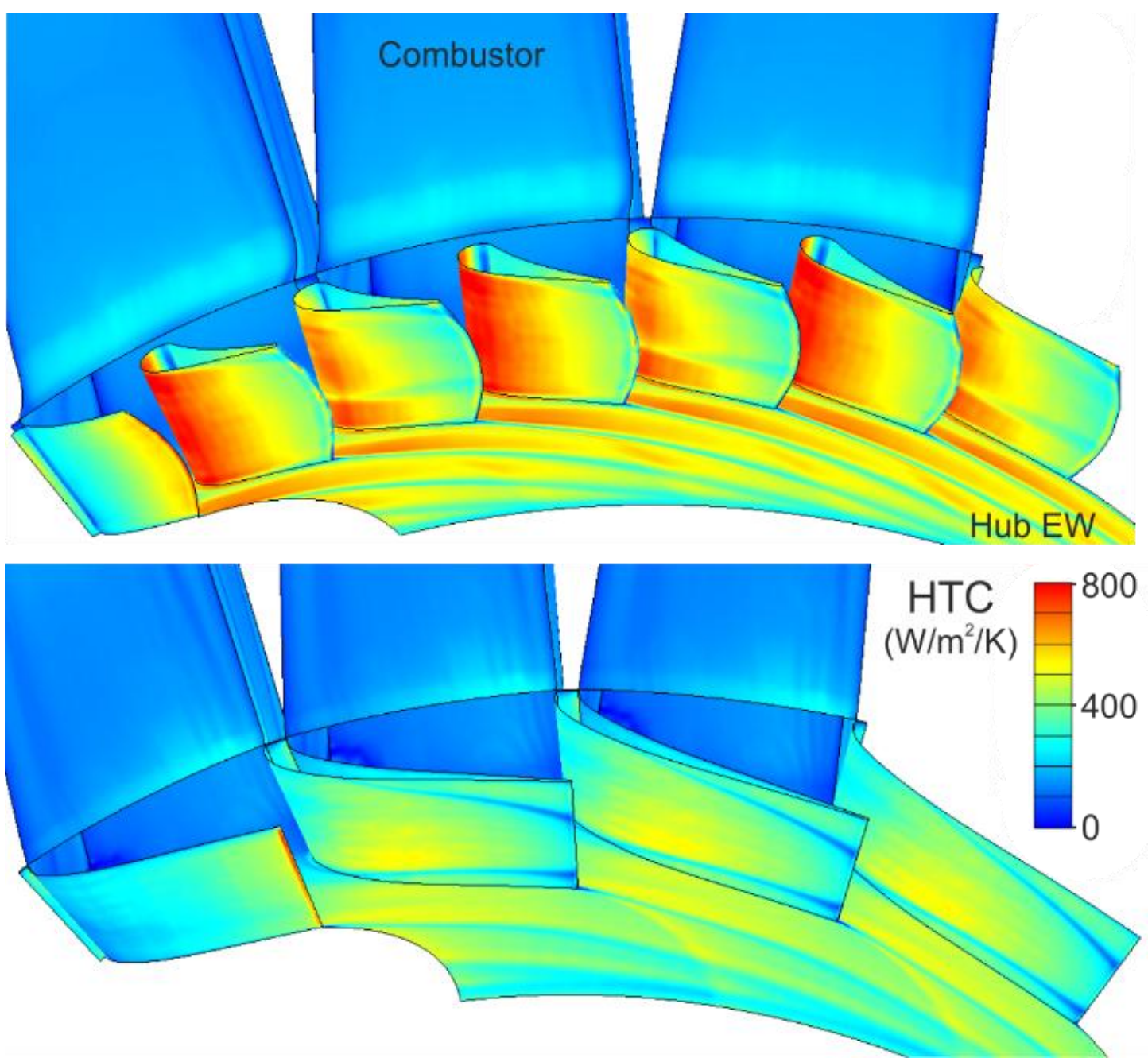

Figure 7-2: Predicted heat transfer coefficient distributions of annular conventional (top) and integrated (bottom) vane domain

The annular HTC distributions are nearly identical to the HTC distributions obtained from linear simulations and agree well with the experimental measurements. A distinct reduction of HTC for the integrated vane concept is evident on vanes and endwalls. The integrated vane does not have a stagnation pressure region as the conventional vane, and peak values thus occur only in the region of peak velocities 
on the suction surface. The boundary layer from the combustor wall is passed onto the integrated vane and thus effectively further reduces HTC levels on the integrated vane surfaces. In addition, the region of thin boundary layer development, found downstream of the passage vortex on the conventional vane endwalls, is absent in the integrated vane design resulting in reduced HTC levels. This phenomenon is congruent to the behaviour depicted by the entropy generation rate and explained schematically by Figure 5-15. Furthermore, the increased radial migration of secondary flows on the suction surface of the integrated vane, indicated by separation lines, can be seen. This is the cause of reduced HTC levels on the integrated vane's suction surface close to the endwall regions.

Averaging HTC and integrating HTC (heat load) over all the wetted surfaces of the same axial distance for both domains results in a reduction of $15 \%$ and $20 \%$ respectively, with a reduced wetted area of $7 \%$. It should further be noted that the modelled flowfield at the combustor-turbine interface is quite realistic since combustors of gas turbines for power generation are based on lean-burn premixed technologies, are increasingly internally cooled using steam or precooled air, and do not have cooling dilution jets. Thus, the state of the BLs at the inlet of this study's domain and real turbines is similar.

\subsection{Stage efficiency}

To evaluate the performance of the full stage, engine realistic boundary conditions (as described in chapter 3) were applied. The effect of the new integrated vane concept on the stage aerodynamic performance is shown in Figure 7-3 in form of lost efficiency and compared to the conventional vane design. The lost efficiency was obtained by using the growth of massflow-weight averaged entropy through the domain, as shown in equation (6). 


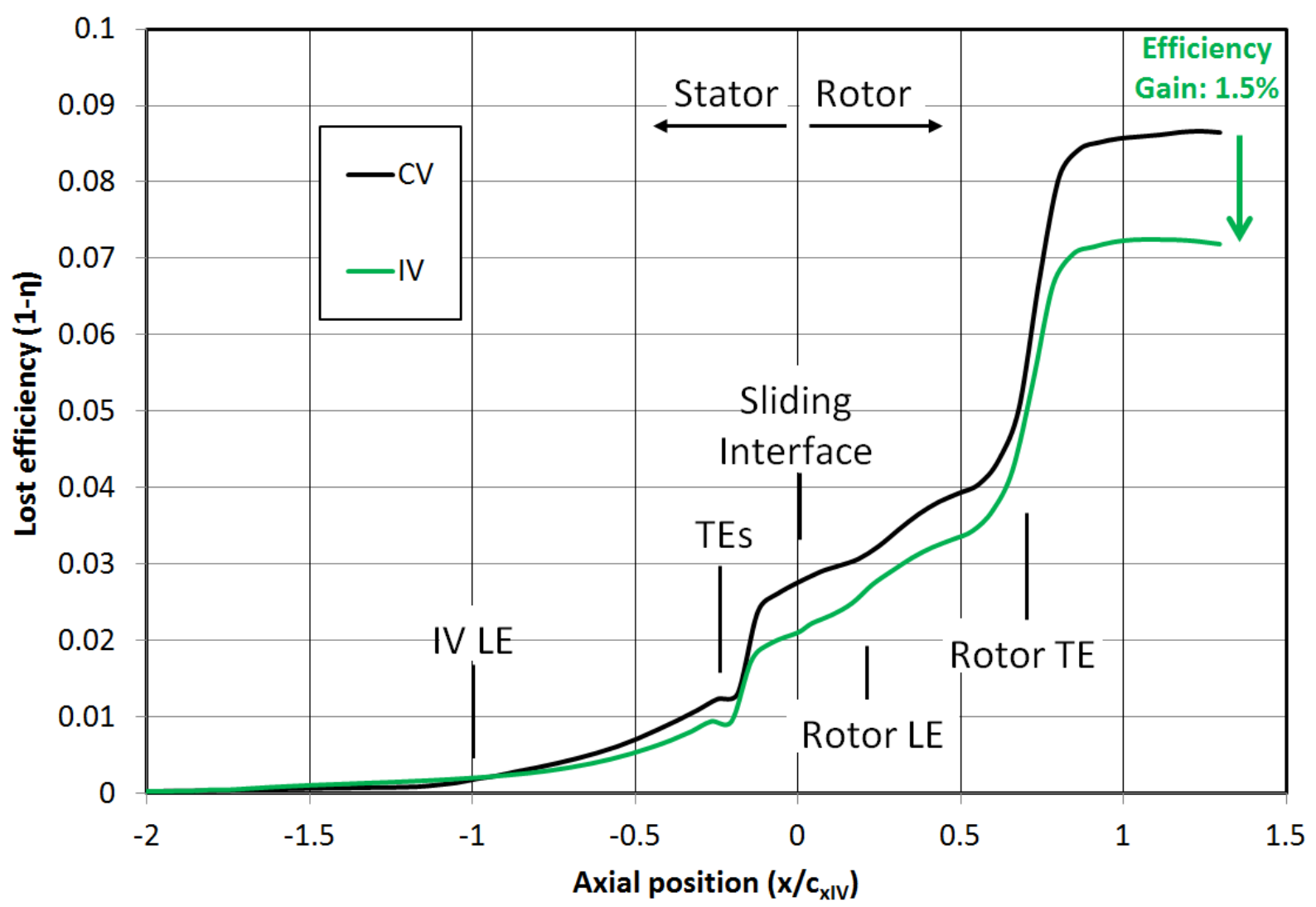

Figure 7-3: Time-averaged efficiency loss through conventional and integrated full stage domains

It can be seen that the integrated vane design outperforms the conventional vane design by $1.5 \%$ at the exit of the domain. This gain can be subdivided into a gain in stage efficiency of approximately $0.7 \%$ and $0.8 \%$ in the stator $\left(-1<x / c_{x I V}<0\right)$ and rotor $\left(0<x / c_{x I V}<1\right)$ respectively. It has been shown (see reduction of total pressure loss coefficient in Figure 5-16) that the former is due to a reduction in profile and endwall loss caused by a reduction in the area of high Mach numbers and boundary layer diffusion on the vane surface, and a halving of the number of wakes. On the rotor side, it can be seen that the lost efficiency stays nearly parallel for conventional and integrated vanes between $0<x / c_{x I V}<0.5$. It is apparent that the slope of lost efficiency through the rotor is slightly lower at the exit and downstream of the rotor for the integrated vane design. 
A better qualitative understanding of this loss generation can be obtained by looking at the instantaneous distribution of the entropy function $\left(\frac{p}{p_{r e f}} \cdot \frac{T}{T_{r e f}}\right)^{\left(\frac{-\gamma}{\gamma-1}\right)}$ within the rotor, which is shown in Figure 7-4 at midspan for conventional and integrated vane domains.

a) Conventional vane stage At midspan

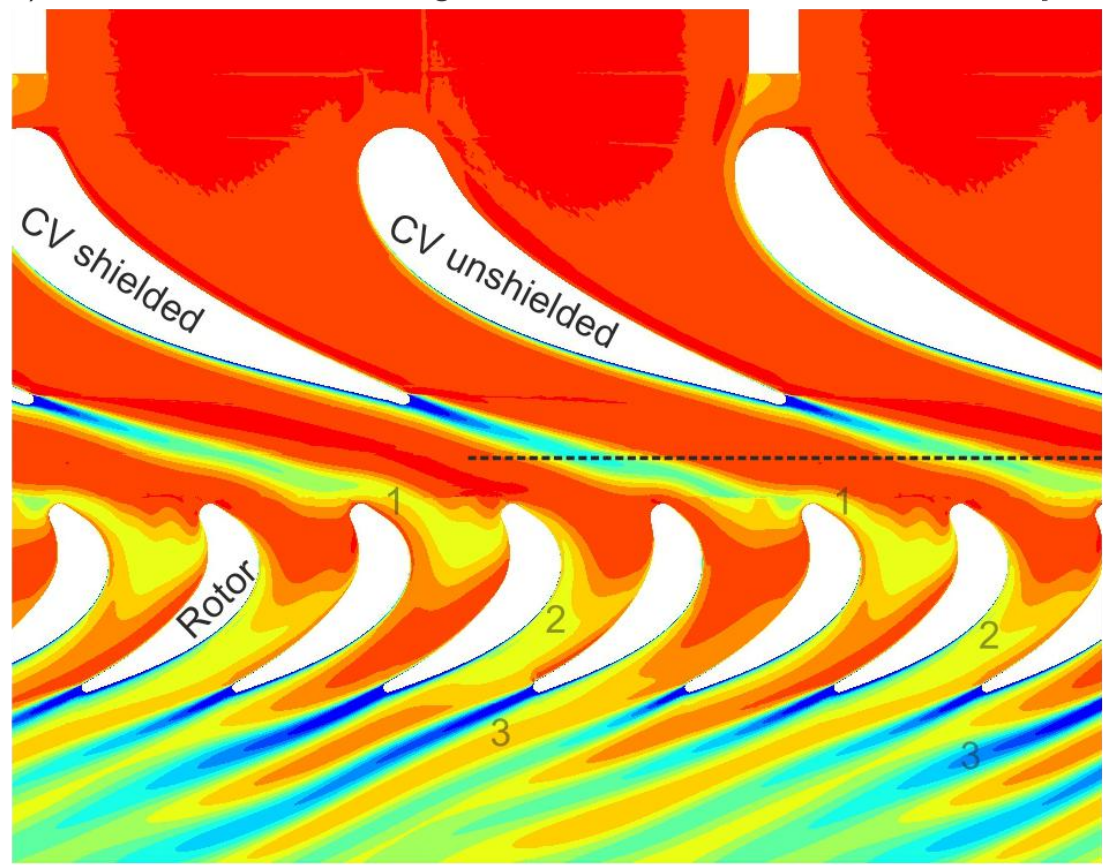

b) Integrated vane stage

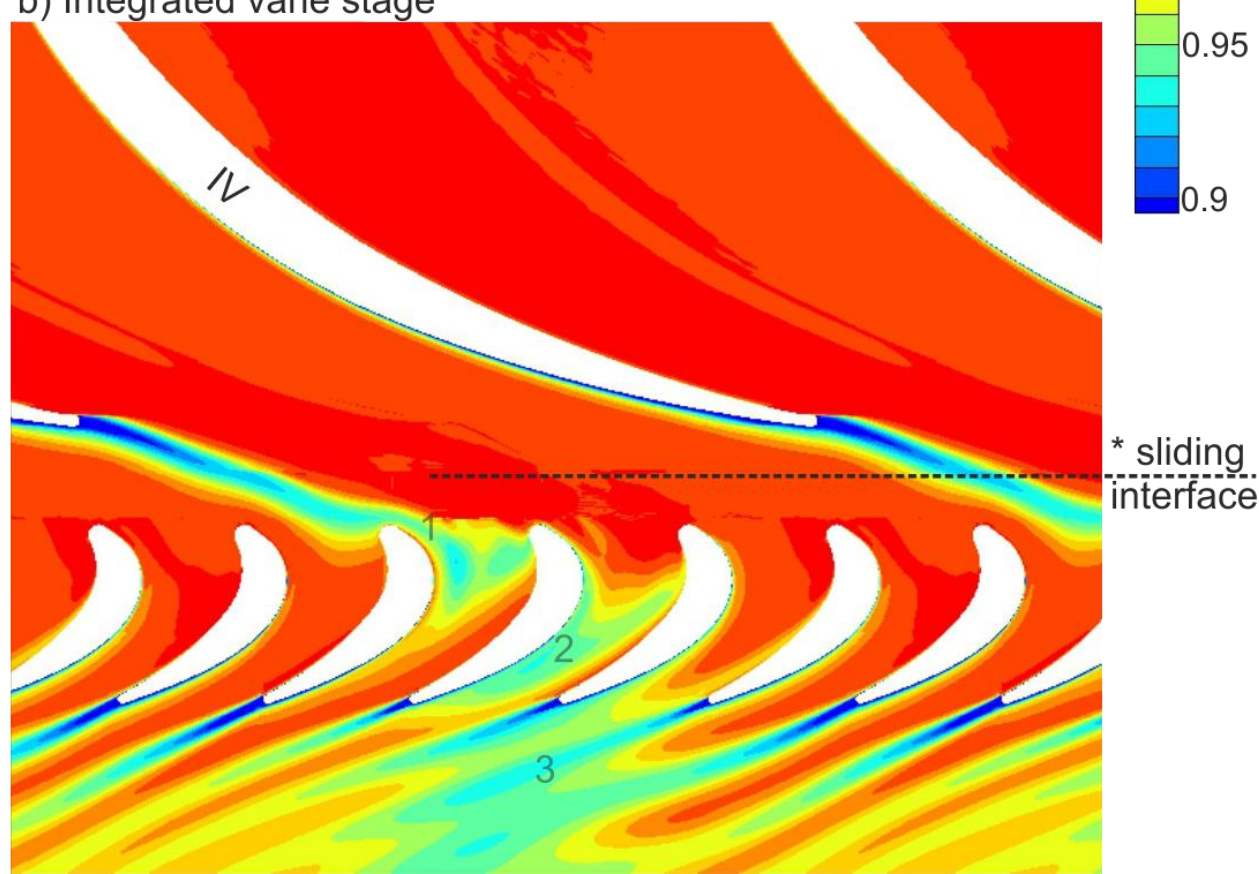

Figure 7-4: Instantaneous entropy function contour of unsteady full-stage CFD for conventional (top) and integrated (bottom) vane design at midspan for no-swirl case 
For the conventional vane design, two wakes per combustor are generated. The shielded conventional vane leads to slightly higher loss (thicker wake, lower entropy function levels) compared to the unshielded conventional vane due to the upstream combustor wall's wake. This can also be seen in Figure 7-5, which shows the instantaneous entropy function distribution at the sliding interface for the conventional and integrated vane design.

a) Conventional vane stage At sliding interface $\left(^{*}\right)$
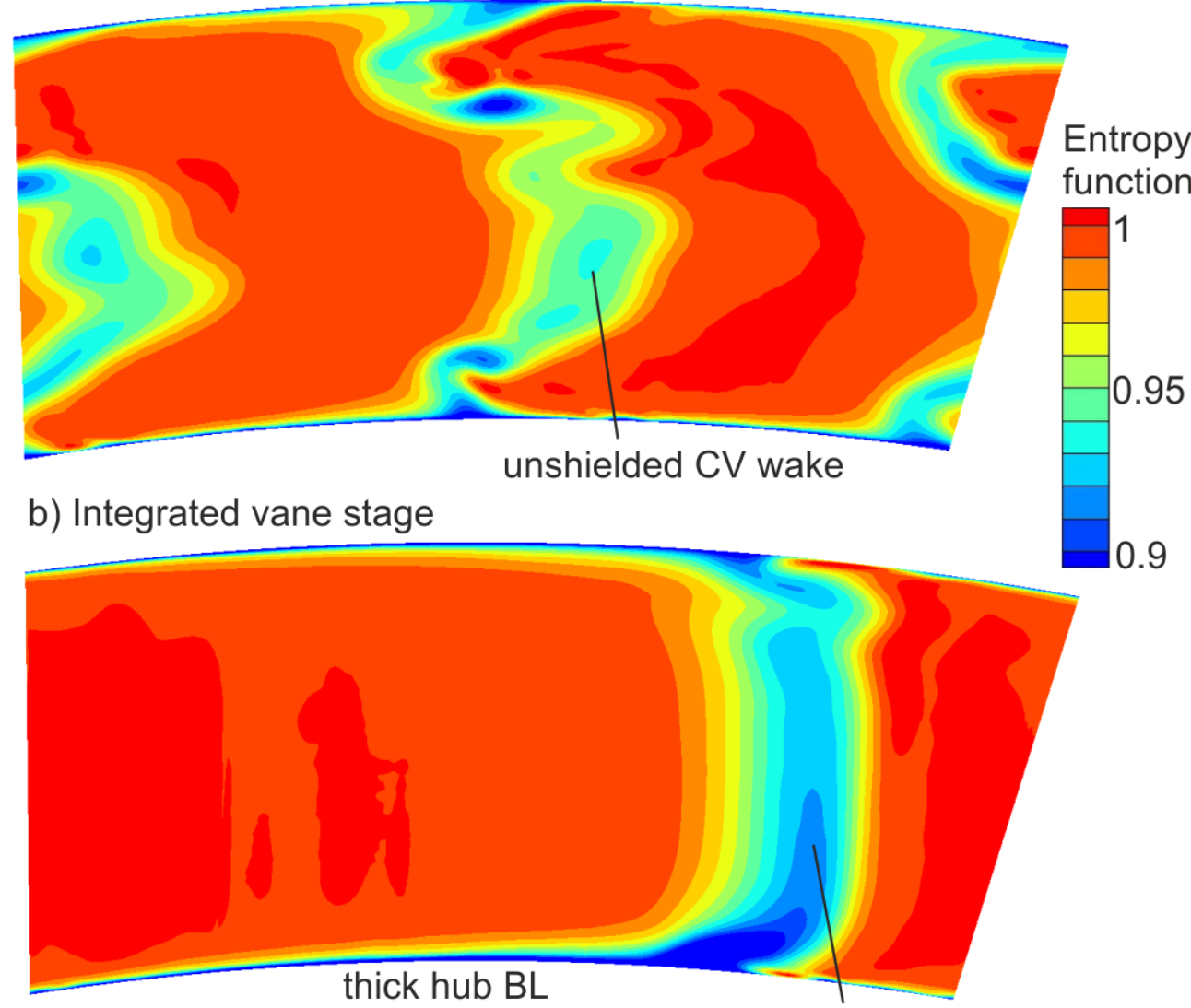

IV wake

Figure 7-5: Instantaneous entropy function at sliding interface for conventional vane

(a) and integrated vane (b) design

The distribution at the sliding interface shows that the conventional vane wakes are skewed due to the compound lean of the vane profile (with increased metal exit angle at midspan and reduced metal exit angle at the endwalls. The wakes become increasingly distorted when approaching the rotor's potential field. The wakes feed into the rotor. This results in losses inside and downstream of the rotor (see steps 1-3 
in Figure 7-4) due to the mixing out of the wakes and due to the interaction of the wake with the rotor's blade boundary layers on pressure and suction surface. The integrated vane design, in comparison, generates only one wake per combustor. While the integrated vane's trailing edge thickness is identical to the conventional vane's trailing edge thickness, its aspect ratio is reduced. The increased axial chord and the transferral of the combustor wall boundary layer onto the integrated vane lead to higher profile loss for one integrated vane compared to one conventional vane. This results in a slightly stronger wake. It can nonetheless be seen that the halving of the number of wakes by the integrated vane design overcompensates the slightly increased wake strength, with clearly lower loss inside and downstream of the rotor compared to the conventional vane design. The distribution at the sliding interface shows that the integrated vane's wake is nearly radial in shape. This is due to the vane's effectively two-dimensional shape, which does not feature any lean or sweep profiling techniques. The wake is thus distributed over a smaller number of rotor passages compared to the conventional vane design, which should be noticeable in the downstream distributions. A look at the endwalls shows that the integrated vane design results in thicker boundary layers due to the removal of the horse-shoe vortex system commonly found in conventional vane passages. Moreover, the boundary layer is slightly thicker at the hub compared to the casing due to the radial pressure gradient (with higher pressures towards the casing than towards the hub). This is likely to influence the rotor's secondary flow system. In order to obtain a better understanding of the aerodynamic stage performance it is important to compare the flowfield at the inlet of the rotor.

The performance of the rotor is influenced by the flowfield downstream of the stator vanes. The most relevant indicators of the flowfield are the total pressure and turning distributions. The effect of former (i.e. wake) on the stage was illustrated in Figure 7-4 
with the help of the entropy function. Figure 7-6 on the other hand shows the spanwise distribution of instantaneous relative turning at the sliding interface (i.e. between stator and rotor) and the rotor inlet metal angle.

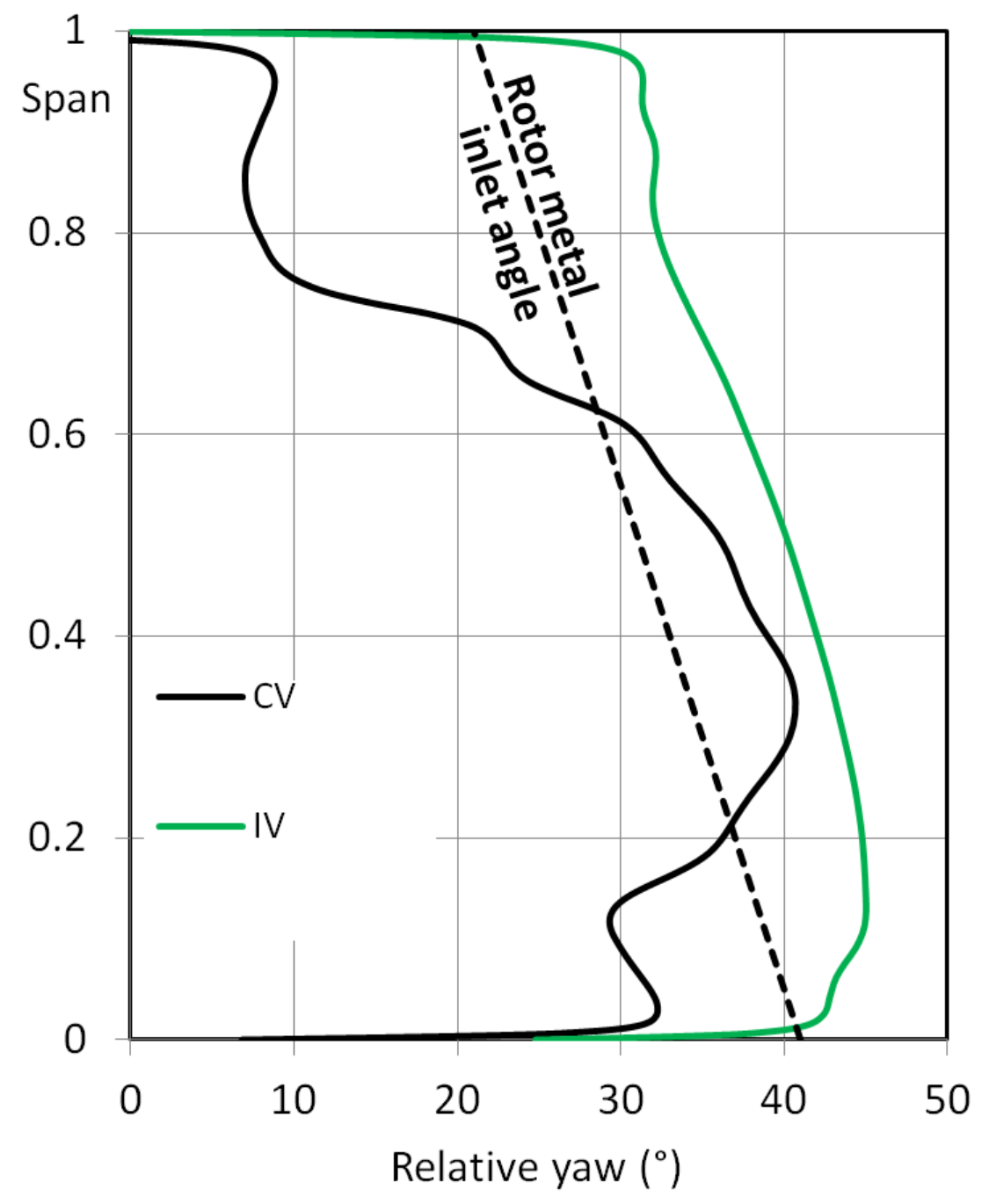

Figure 7-6: Instantaneous relative turning at sliding interface for no-swirl case

Relative turning drops significantly close to the endwalls due to the low axial velocities in this region. As a result the rotors rotational speed has a significant influence on the relative turning in this region $\left(\right.$ since $\left.Y a w_{r e l}=\operatorname{atan}\left(\frac{v_{t}-u}{v_{x}}\right)\right)$. In general, relative turning is lower towards the casing due to the larger radius at constant rotational speed of $3000 \mathrm{rpm}$. Overall the graph shows higher relative turning for the integrated vane. It can be seen that the turning distribution downstream of the integrated vane features significantly lower angle deviations from the rotor inlet metal 
angle between approximately $0 \%-20 \%$ and $65 \%-100 \%$ of span compared to the turning distribution downstream of the conventional vane. This reduction in rotor incidence leads to a smaller penalty of the rotor's aerodynamic performance.

The spanwise distribution presented in Figure 7-6 does not provide information about the pitchwise variation in turning. Since the rotor has a fixed metal inlet angle for each radial profile, a closer look at the pitchwise flow variations is important for understanding the stage performance. Figure 7-7 thus shows the conventional vane rotor instantaneous streamtraces, and inlet total pressure and relative yaw at the hub and casing endwall. The streamtraces indicate negative incidence at the casing endwall. Furthermore, pitchwise variations in relative turning between approximately $20^{\circ}$ to $40^{\circ}$ can be seen at the hub endwall. The total pressure contour also shows a relatively thin and constant boundary layer thickness at the rotor inlet hub and casing endwall, with only slightly lower levels of total pressure behind the conventional vane wakes. The rotor shows relatively tolerant incidence behaviour: The observed range of inlet angles does not significantly alter the secondary flow system. The secondary flow seen on the rotor suction surfaces is - as a result of the rotor's tolerant incidence behaviour and relatively homogeneous boundary layer thickness - also comparable between the rotor passages, and similar for hub and casing.

Figure $7-8$ shows the respective streamtraces, total pressure and relative yaw plot for the integrated vane's rotor. It can be seen that the pitchwise variation in relative incidence at the hub and casing endwall of the integrated vane rotor is significantly higher than for the conventional vane rotor, ranging from approximately $-40^{\circ}$ to $65^{\circ}$. This large variation exceeds the rotor's incidence flexibility and the horse-shoe vortex system differs significantly between the rotor passages. 

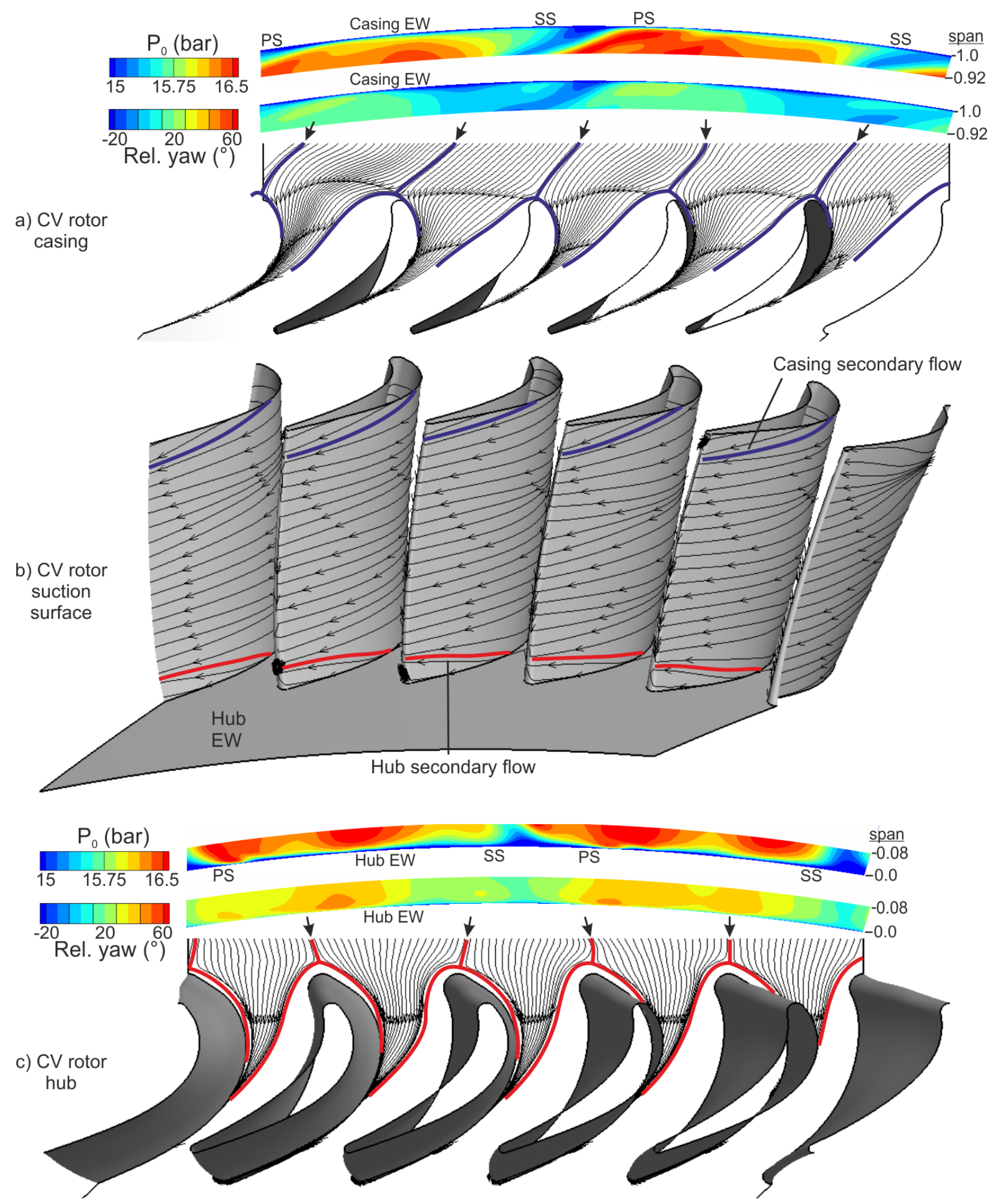

Figure 7-7: Conventional vane rotor instantaneous streamtraces and inlet total pressure \& relative yaw at hub (a) and casing (c) endwall, and instantaneous streamtraces on conventional vane rotor suction surfaces (b) 

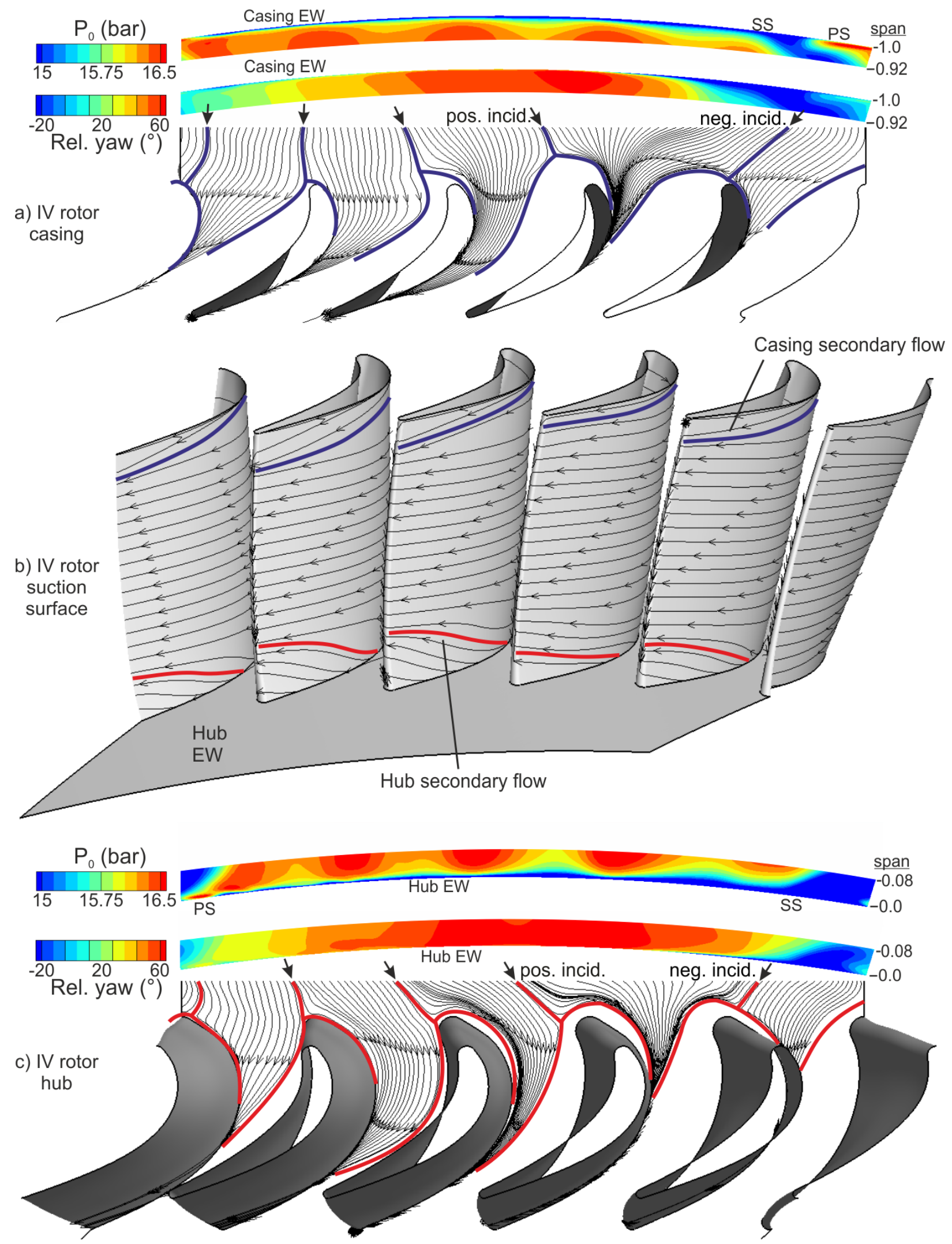

Figure 7-8: Integrated vane rotor instantaneous streamtraces and inlet total pressure \& relative yaw at hub (a) and casing (c) endwall, and instantaneous streamtraces on integrated vane rotor suction surfaces (b) 
The total pressure plots show that the boundary layer is thinner at the casing compared to the hub due to the higher pressures at the casing caused by the radial pressure gradient of the annular design. This results in stronger hub than casing secondary flow on the integrated vane rotor suction surface. It can furthermore be seen that the hub and casing endwall boundary layer thickness increases continuously across the pitch towards the integrated vane suction side. This leads to a higher non-uniformity between the rotor passages secondary flows compared to the conventional vane rotor secondary flows.

The altered number of wakes, and the differing span- and pitchwise turning distribution of the integrated vane design compared to the conventional vane design affect the rotor's aerodynamic performance and a look at the downstream distributions should verify the differences in loss. Figure 7-9 thus shows an axial cut of the instantaneous entropy function downstream of the rotor for conventional and integrated vane design. It can be seen that the integrated vane design results in a pitchwise less uniform distribution of the entropy function due to the halving of the vane count. The wake of the integrated vane is passed through the rotor and leads to an increased loss downstream of rotor passage 4 (see also location ' 3 ' in Figure 7-4b). A relatively clean flow can be seen downstream of the remaining rotor passages $1,2,3$, and 5 (see areas marked with ' + '). The total pressure distribution at the hub endwall in Figure 7-8 (see also Figure 5-15 and Figure 6-6) showed a thickening of the boundary layer towards the integrated vane's suction side. This thicker boundary layer gives rise to increased secondary flows at the rotor hub resulting in the pronounced loss cores downstream of the rotor. This effect is significantly stronger at the hub than at the casing due to the radial pressure gradient of the annular configuration (with reduced and increased static pressure at the hub and casing respectively), resulting in thicker boundary layers at the hub endwall 
compared to the casing endwall upstream of the rotor. The reduced turning towards the integrated vane's pressure side (to the right of the integrated vane's wake) increases the loading of the rotor and leads to higher profile loss (see wakes of the passage 1 rotor blades). The profile loss downstream of the remaining rotors is decreased (see areas marked by '*') resulting in thinner wakes compared to the conventional vane rotor wakes.

a) Conventional vane stage rotor
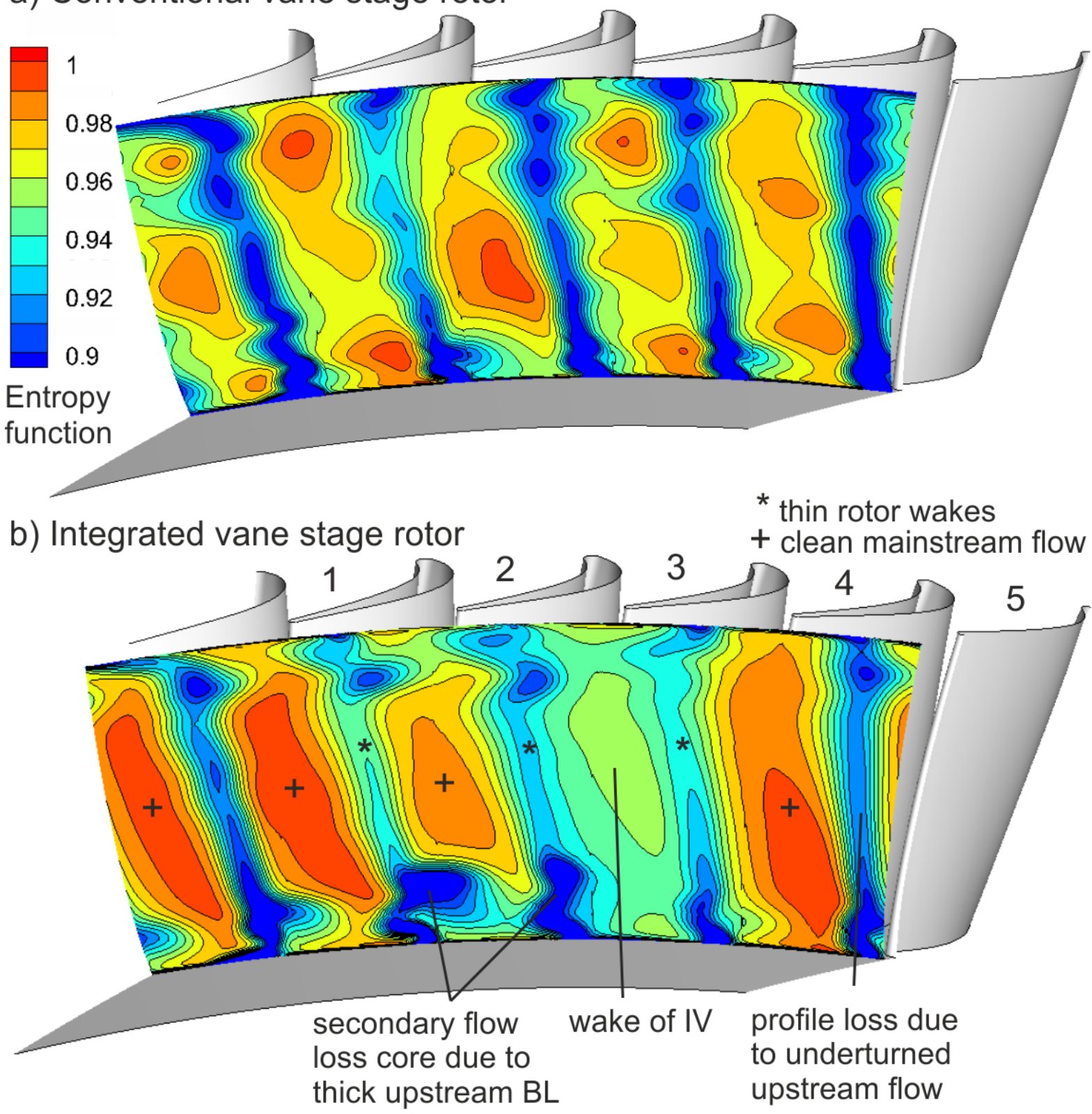

Figure 7-9: Axial cut of instantaneous entropy function downstream of rotor for conventional (top) and integrated (bottom) vane design for a no-swirl case 
In comparison, overall levels of the entropy function are lower for the conventional vane's design, indicating higher loss. The conventional vanes wakes span over a larger circumferential distance compared to the integrated vane (since the former use compound lean towards the trailing edge while the latter effectively has a twodimensional profile) and become increasingly distorted when approaching the rotor. Each wake is thus distributed over a larger number of rotor passages so that they cannot be clearly identified downstream of the rotor.

Time- and pitchwise-averaging the entropy function distributions at the rotor inlet (at the sliding interface) and outlet (at the exit of the domain), as seen in Figure 7-10, allows for a more quantitative comparison. Levels of the entropy function are higher at the rotor inlet for the integrated vane except for the region close to the hub endwall (due to the integrated vane's thicker endwall boundary layer and more pronounced loss cores). This is due to the integrated vane's lower profile and trailing edge loss. The entropy function levels are also higher at the rotor outlet for the integrated vane between $25 \%-100 \%$ of span. Entropy function levels are similar for both designs at the rotor outlet between $0 \%-25 \%$ of span due to the integrated vane's higher hub secondary flow loss. 


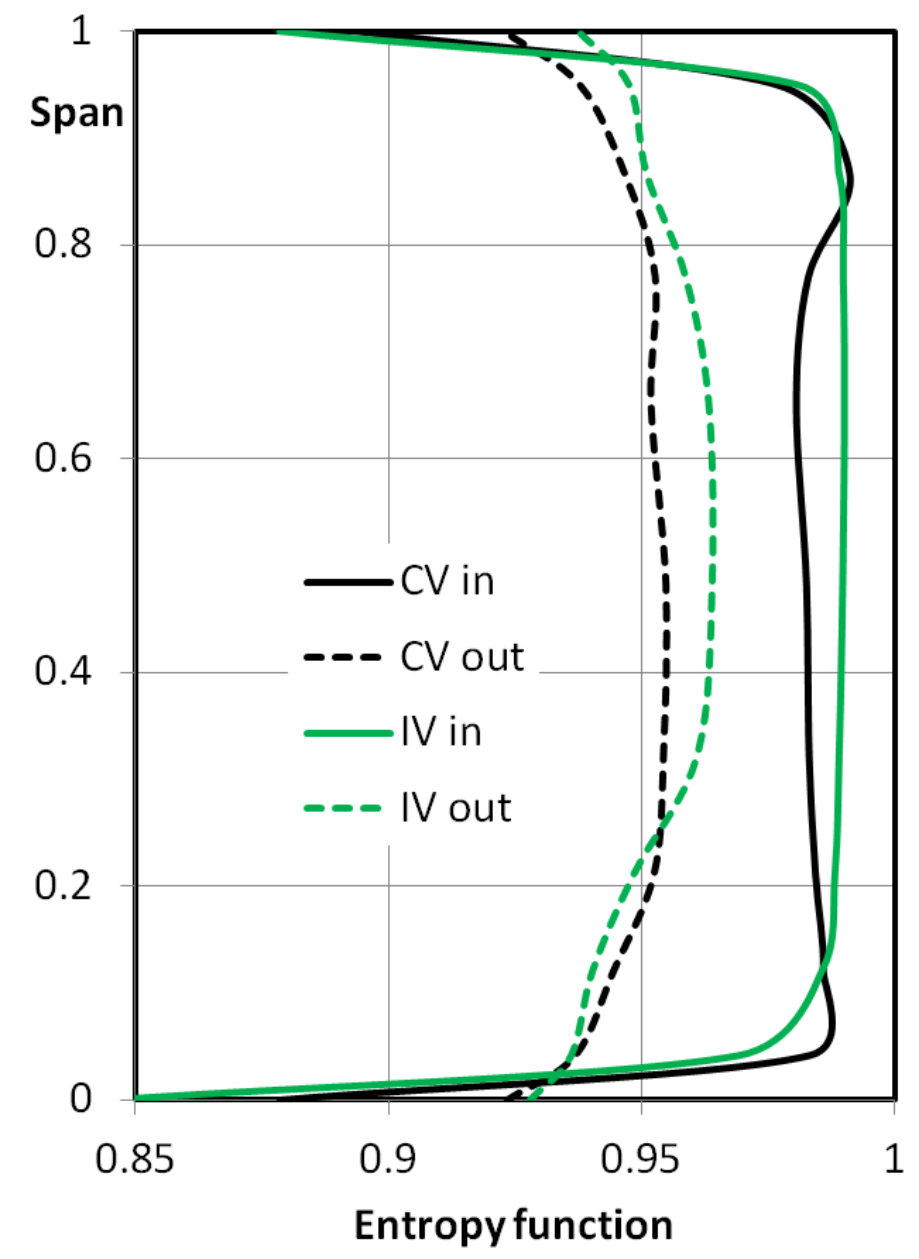

Figure 7-10: Time- and pitchwise-averaged entropy function at rotor inlet and outlet for a no-swirl case

It thus seems that the altered flow distribution downstream of the integrated vane (see Figure 5-11), and thus the altered incidence at the inlet of the rotor, does not detrimentally affect the aerodynamic performance of the rotor. On the contrary, the rotor for the integrated vane design outperforms the rotor for the conventional vane design due to the halved number of incoming wakes as well as the decreased pitchwise averaged rotor incidence angle penalty. A gain in stage efficiency of approximately $1.5 \%$ for the integrated vane design compared to the conventional vane design at the exit of the stage domains is reached. As the simulations for the conventional and integrated vane domains were performed with identical rotors, which were optimized for a conventional vane design, further aerodynamic benefits might be achieved by a full-stage design optimization. 


\section{Chapter 8: Influence of Swirl on Integrated Vane}

\subsection{Abstract}

This chapter focuses on an experimental and numerical investigation into the effect of combustor swirl on the integrated vane's performance. The flowfield distributions downstream of the combustor transition duct are obtained for a swirl and a reversed swirl case using aerodynamic traverse measurements. Downstream measurements show a slight skewing of the integrated vane's wake due to swirl and a pronounced spanwise variation in turning. The conventional vane design leads to smaller variations in the downstream flowfield distributions due to the passage central vane that helps to homogenize the flow. It is furthermore shown that swirl leads to only a minor shift in the heat transfer coefficient distributions. Finally it is illustrated that swirl affects the rotor performance more strongly for an integrated vane design than for a conventional vane design. The non-uniformity of turning downstream of the integrated vane due to swirl leads to changes in rotor incidence and loading. Despite a stage efficiency penalty of $1.2 \%$ due to swirl for the integrated vane design, it still outperforms the conventional vanes by $0.7 \%$. In order to mitigate the pronounced effect of flow non-uniformity due to swirl it is suggested to use vane profiling. It is hereby shown that further stage performance gains are possible by homogenizing the turning downstream of the integrated vane. An overview of the results presented in this chapter is given by Jacobi and Rosic (2016) (2018). 


\subsection{Aerodynamics upstream of the vane}

Combustors nowadays use swirl generators to create a lean mixture of air and fuel prior combustion for a cleaner operation with less emissions. The thesis so far only investigated a no-swirl case, representative of the upstream turbulence grid. This chapter will illustrate the effect of combustor swirl on the integrated vanes aerodynamic, thermal and stage performance. To do this, the flowfield generated downstream of the swirlers needs to be characterised first. Thus, aerodynamic traverse measurements using a five-hole-probe were obtained at the exit of the combustor transition duct. Figure 8-1 shows the total pressure $\overline{p_{0}}$, yaw and pitch angle distributions downstream of the transition duct obtained through traverse measurements with the two different swirlers (swirl case and reversed swirl case) installed upstream. The distributions are obtained through interpolation between the 570 measurement locations. A pronounced low-pressure core in the centre is apparent, which represents the swirlers wake. Furthermore, areas of high total pressure in the top left and bottom right corners can be seen for the swirl case. The white arrows in the yaw and pitch contours indicate the global velocity direction in this region. Combining both plots illustrates the residual swirl. The swirl case thus leads to positive yaw (in the direction of the vane turning) in the upper half (with peak levels exceeding $30^{\circ}$ ) and to negative yaw in the lower half of the domain (with minimum levels below $-30^{\circ}$ ). The azimuthal velocity of the residual swirl leads to a pressure gradient $\frac{d p}{d r}=\frac{\rho u_{\theta}}{r}$ due to centrifugal forces and thus to a low static pressure core (similar to the low total pressure core in Figure 8-1) in the centre. Traverse measurements of the reversed swirl case show that the distributions are nearly identical when mirroring the swirl distributions on a vertical axis and switching the velocity directions for pitch and yaw (i.e. rotating each velocity vector by $180^{\circ}$ ). 


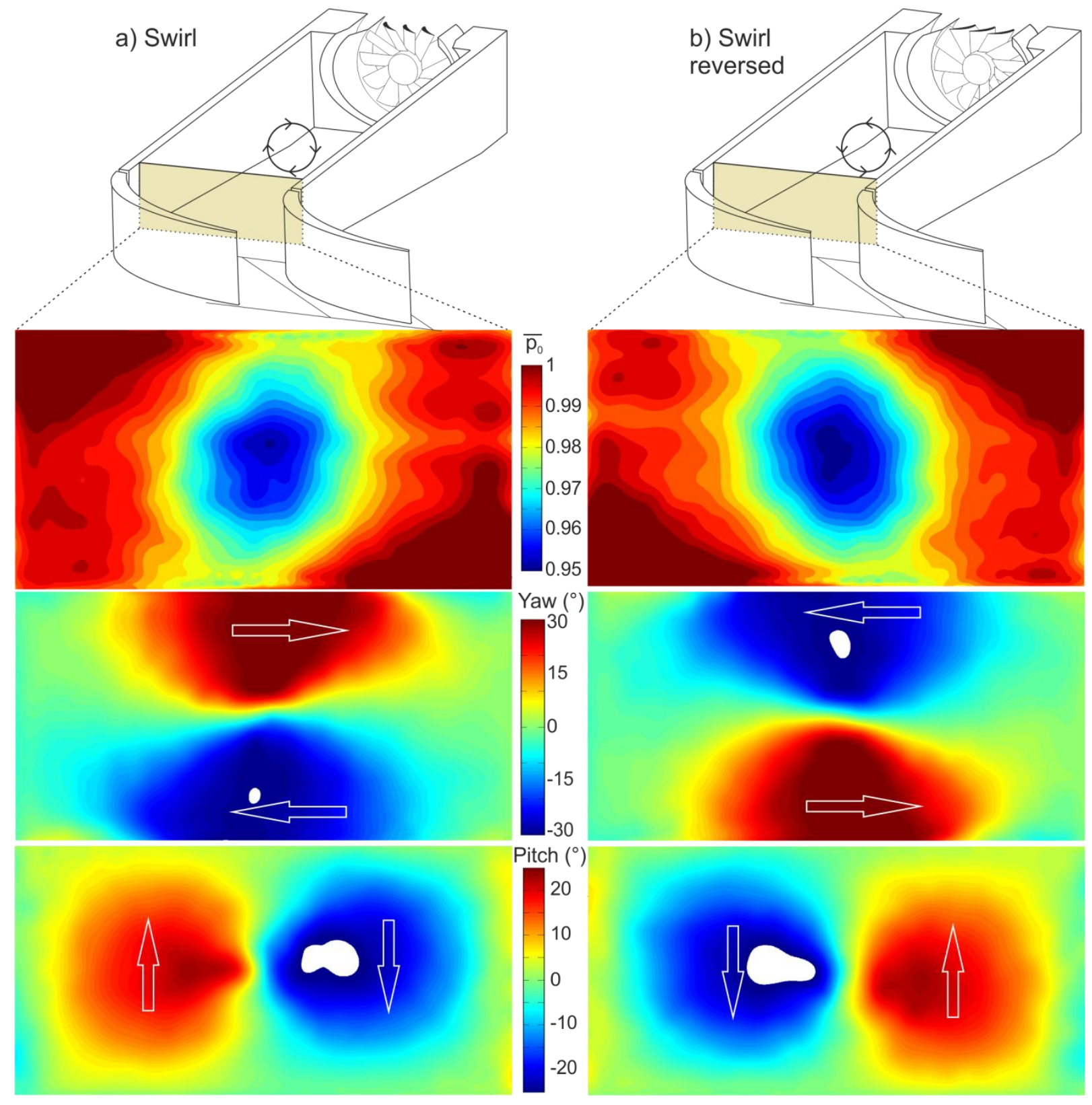

Figure 8-1: Measured dimensionless total pressure (top), yaw (middle) and pitch (bottom) distributions downstream of the transition duct (vanes not present) for a swirl (a) and a reversed swirl (b) case

\subsection{Aerodynamics downstream of the vane}

The residual swirl and low pressure core (subsequently referred to as residual swirl core) at the exit of the combustor transition duct also modify the downstream aerodynamic distributions. Figure $8-2$ shows the total pressure in an axial plane $15 \%$ of integrated vane axial chord downstream of the integrated vane for a no-swirl, a 
swirl and a reversed swirl scenario. While distributions are clearly skewed due to the influence of swirl, overall magnitudes remain similar. It can be seen that the low pressure residual swirl core is slightly convected towards the hub for a swirl scenario, and towards the casing for a reversed swirl scenario.

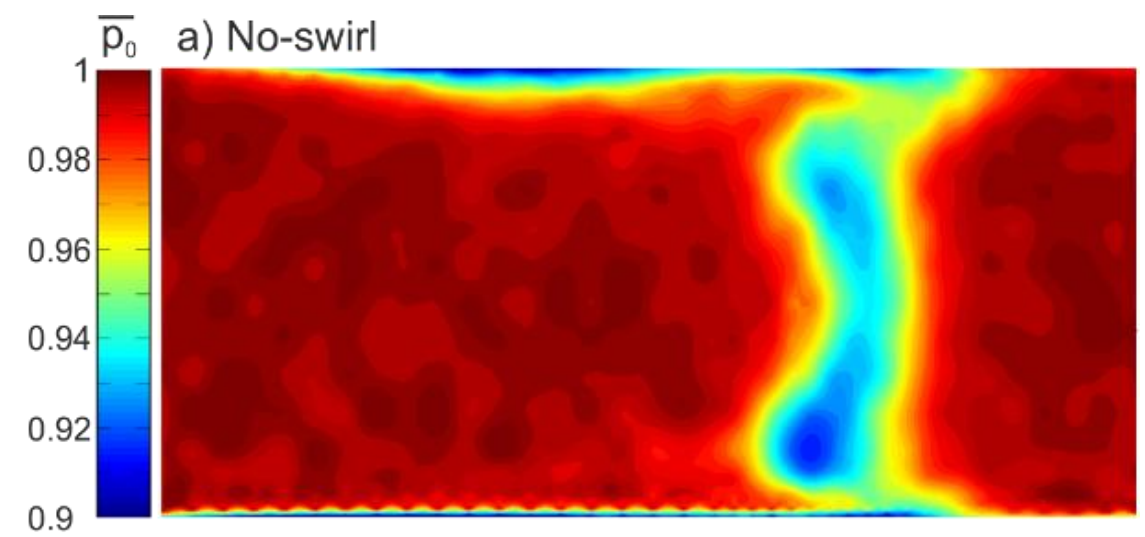

b) Swirl

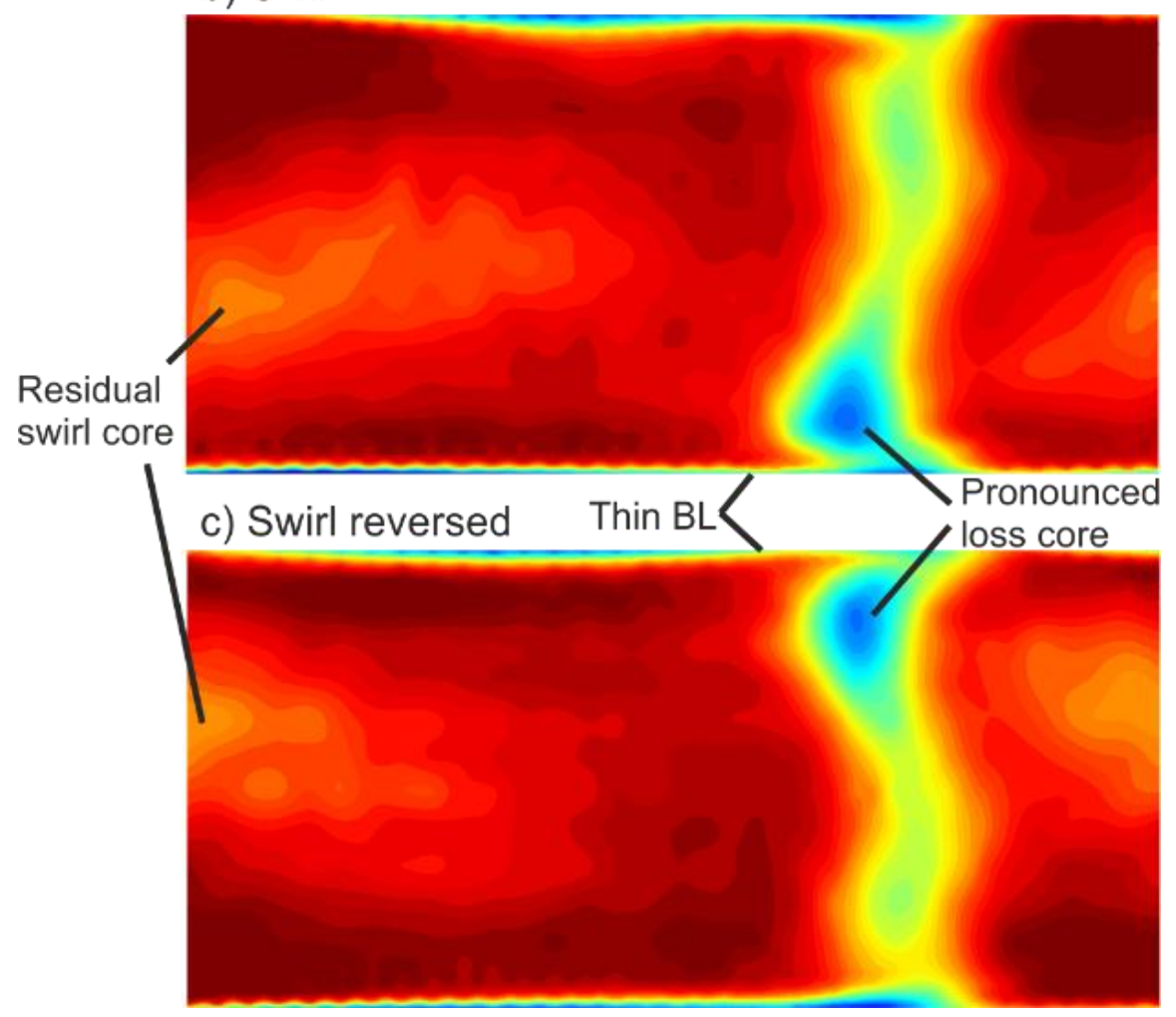

Figure 8-2: Traverse measurements downstream of integrated vane showing dimensionless total pressure distributions for a no-swirl (top), swirl (middle) and reversed swirl (bottom) setup

The wakes of the integrated vane are skewed by the swirl. In the swirl case, the negative upstream yaw towards the hub (see Figure 8-1) leads to an increased vane 
loading at the hub which results in increased secondary flows and thus a pronounced hub loss core. Towards the casing, upstream yaw is positive, leading to reduced vane loading, a reduction in casing secondary flow and thus a reduction of the casing loss core. Similarly, the reversed swirl case leads to a pronounced casing loss core and reduced hub loss core.

Figure 8-3 shows the turning downstream of the integrated vane.

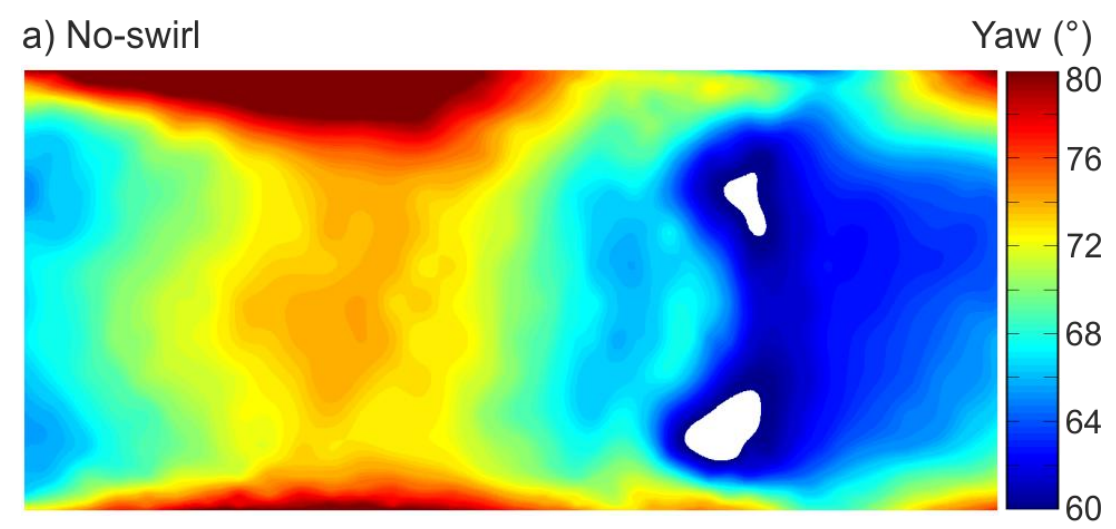

b) Swirl

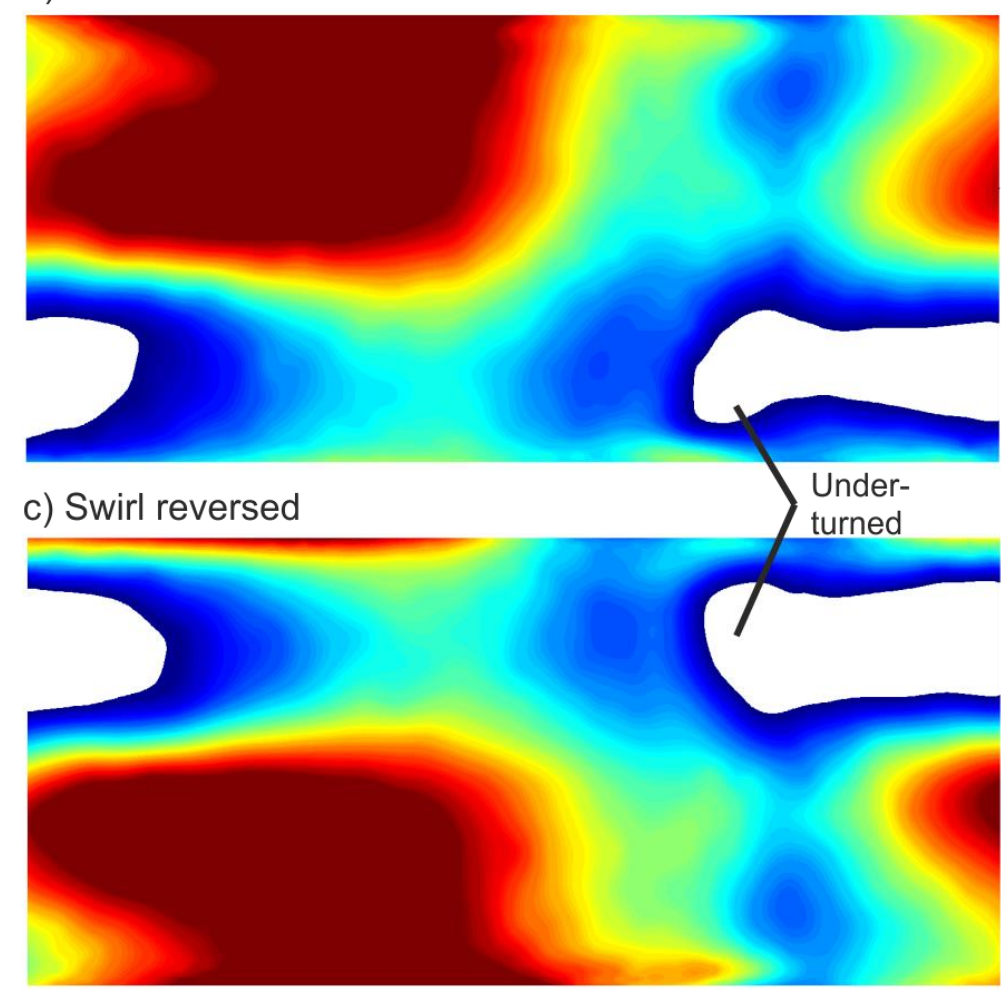

Figure 8-3: Traverse measurements downstream of integrated vane showing yaw distributions for a no-swirl (top), swirl (middle) and reversed swirl (bottom) setup

While the no-swirl case leads to a horizontally symmetric yaw distribution, the swirl case leads to distorted turning with increased turning towards the casing and 
reduced turning towards the hub (due to the negative upstream yaw at the hub and positive upstream yaw at the casing in Figure 8-1). Opposite trends are observed for the reversed swirl case with decreased turning towards the casing and increased turning towards the hub.

Figure 8-4 shows the numerical predictions for the turning downstream of the integrated vane for the three cases.

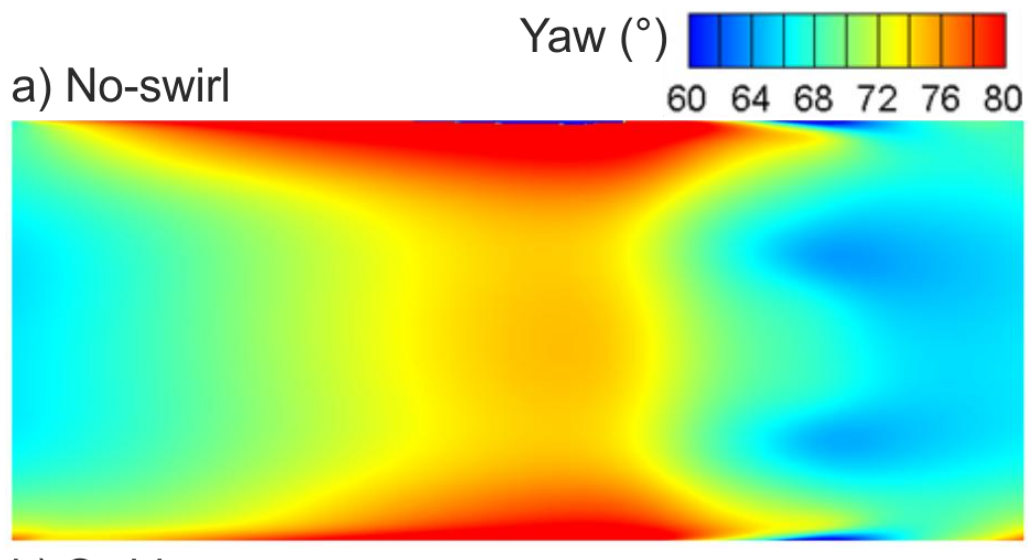

b) Swirl

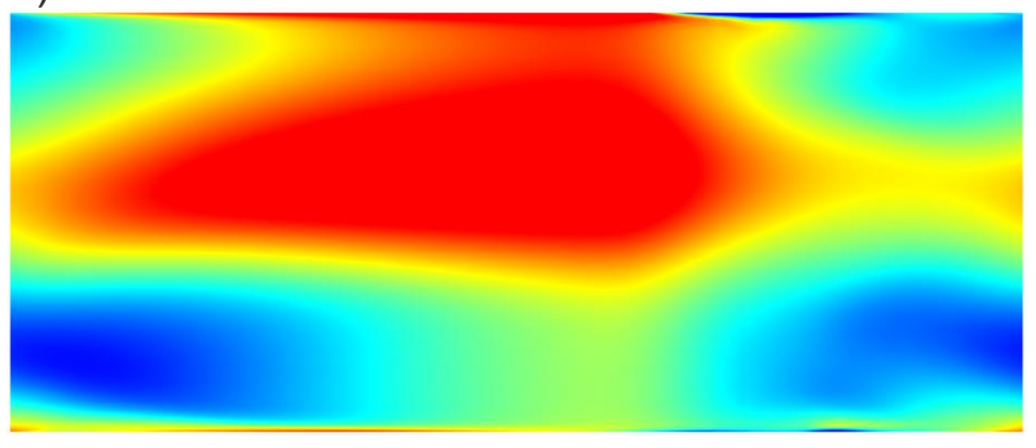

c) Swirl reversed

Figure 8-4: Predicted turning for a no-swirl (a), swirl (b) and reversed swirl (c) case

It can be seen that overall qualitative agreement is very good for all cases. Nonetheless it is apparent that regions of very high turning are underpredicted while regions of very low turning are overpredicted. Pitchwise averaging dimensionless 
total pressure and yaw, as seen in Figure 8-5, allows for a more quantitative comparison.
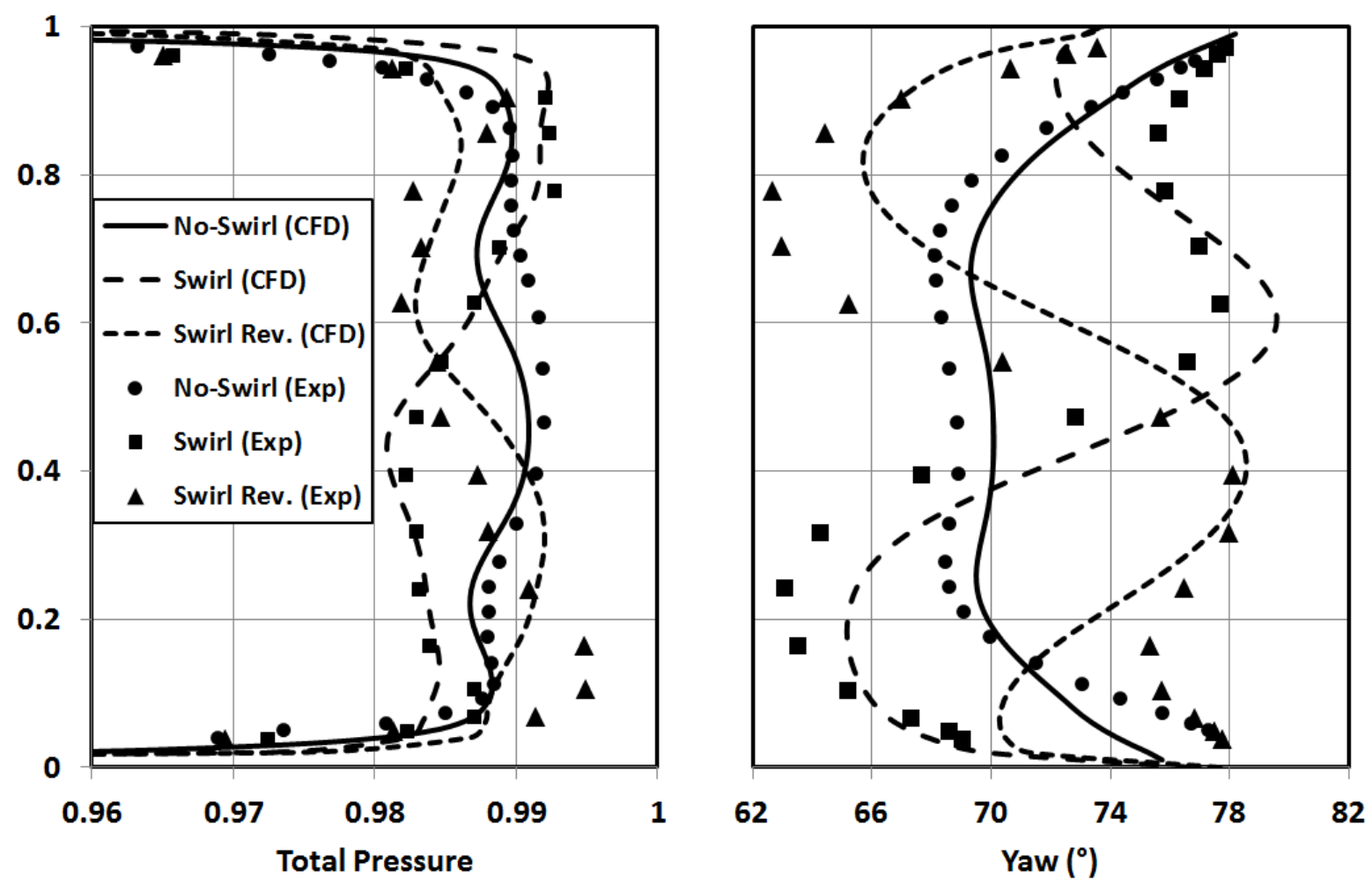

Figure 8-5: Pitchwise averaged dimensionless total pressure (left) and yaw (right) downstream of the integrated vane for a no-swirl, swirl and reversed swirl scenario; dots and lines represent experiments and simulations respectively

The no-swirl scenario results in symmetric total pressure and yaw distributions. The two loss cores of the wake have the same magnitude and an equal distance to the hub and casing endwalls. The flow close to the endwalls is overturned and flow around midspan is slightly underturned, with an average turning of $70.5^{\circ}$. The swirl scenario in comparison results in decreased loss and increased turning in the upper half of the domain, and in increased loss (due to more pronounced loss core and residual swirl core) and decreased turning (due to negative upstream yaw) in the lower half of the domain. The opposite is the case for the reversed swirl scenario. Averaging yaw for both swirl scenarios results in a turning of $71.4^{\circ}$. Overall agreement between experiments and CFD is found to be good for the dimensionless 
total pressure. The predicted turning captures the trends correctly while its quantitative agreement is suboptimal for the swirl cases. A likely reason for this is the fact that the upstream traverse measurements were obtained in the absence of the downstream vanes. This changes the back pressure and ignores the fact of the vanes' potential field extending upstream into the transition duct. Better agreement might thus be achieved by altering the working section design to allow upstream traverse measurements with vanes in their usual location. Since these measurements were used as the CFD inlet boundary condition, additional discrepancies will be caused by this limitation. Moreover it is possible that the mixing length approach is not capable of accurately predicting the complex mixing processes of swirling flows and large structures in the freestream. For this study it was however decided that the trends are captured to a satisfactory extent.

Figure 8-6 shows the predicted flowfield inside and downstream of the integrated vane passage. An axial cut of static pressure (grey-scale) illustrates a slight static pressure gradient in spanwise direction. This forces the residual swirl core (illustrated by iso-surface of total pressure) closer to the hub endwall and allows more of the hub endwall boundary layer fluid to migrate onto the suction surface (compared to a noswirl case with no radial pressure gradient). The suction surface streamtraces also indicate a radial velocity component towards the hub endwall. Both flows (i.e. from the suction surface and from the hub endwall) meet and form the more pronounced hub loss core for the swirl case. The early onset of endwall fluid migration onto the suction surface also leads to a thin boundary layer on the hub endwall close to the suction surface (also indicated in Figure 8-2b). The opposite behaviour is observed for the reversed swirl case and analogous reasoning can be applied. Good qualitative and quantitative agreement of the dimensionless total pressure downstream of the 
integrated vane between experiments (see Figure 8-2) and the predicted distributions is noted.

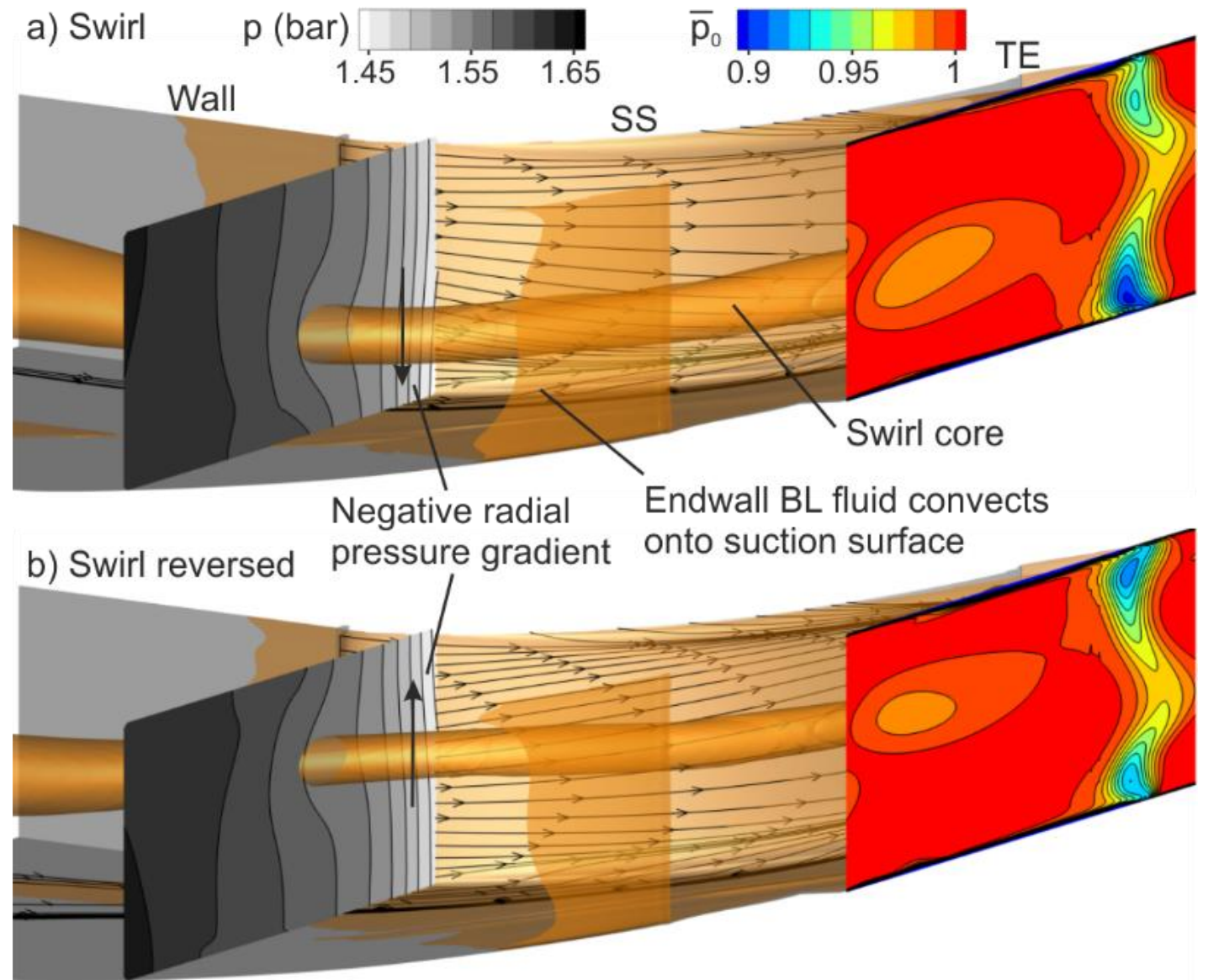

Figure 8-6: Predicted dimensionless total pressure downstream of the integrated vane with swirl (a) and reversed swirl (b); axial cut of static pressure; iso-surface of total pressure; streamtraces on suction surface

In order to assess the effect of swirl on the integrated vane's aerodynamic performance it is useful to draw a comparison to the conventional vane's aerodynamic performance with swirl. Figure 8-7 thus shows aerodynamic traverse measurements with total pressure and yaw distributions downstream of the conventional vanes with upstream swirl. It can be seen that the form of the shielded vane's wake (which has a higher loss compared to the unshielded vane's wake due to the upstream combustor wall) is nearly symmetrical. The unshielded vane in contrast seems to be more affected by the swirl. It's hub loss core is larger in size and is convected less in pitchwise direction (further left) due to the negative upstream yaw in the bottom half of the domain (see Figure 8-1a). It can also be seen that the 
high total pressure zones in the top left and bottom right corners of Figure 8-1a are convected through the vane passages and can still be seen downstream of the vanes. The yaw distributions show that turning towards the hub is slightly lower than the turning towards the casing, as expected. Besides these aspects, the total pressure and yaw distributions seem similar to the no-swirl traverse measurements shown in Figure 5-8 and Figure 5-9.

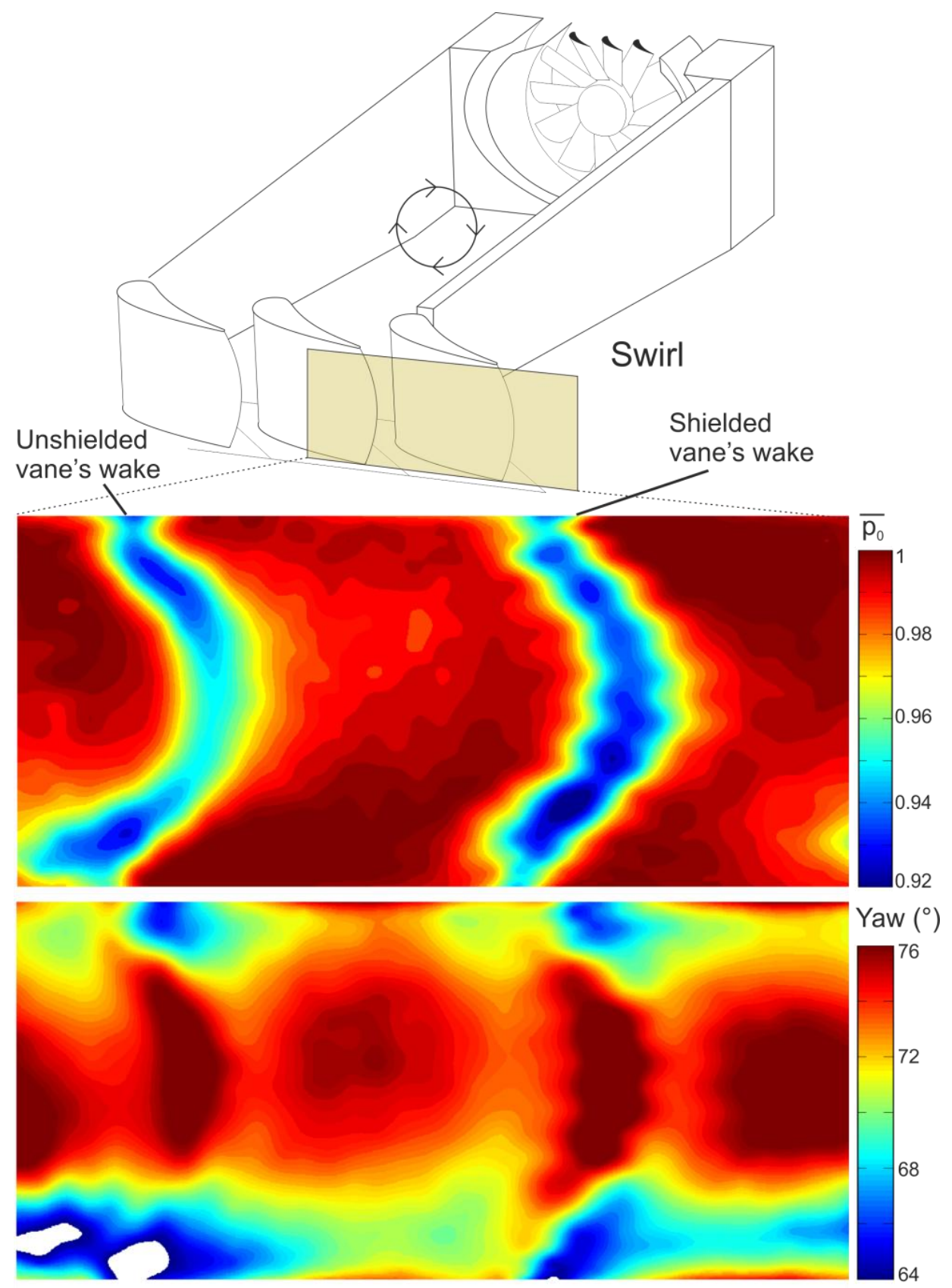

Figure 8-7: Traverse measurements downstream of conventional vanes showing dimensionless total pressure (top) and yaw (bottom) distributions for a swirl setup 
The pitchwise averaged total pressure and yaw distributions in Figure 8-8 allow a more detailed quantitative comparison between the swirl and no-swirl cases. The increased hub secondary flows (caused by the increased vane loading due to the negative upstream yaw) result in stronger hub loss cores. Overall, losses are higher except in the casing loss cores which are decreased due to the reduced vane loading caused by the positive upstream yaw. Furthermore it can be seen that turning is decreased by approximately $2^{\circ}$ and $1^{\circ}$ at $15 \%$ and $90 \%$ of span respectively.

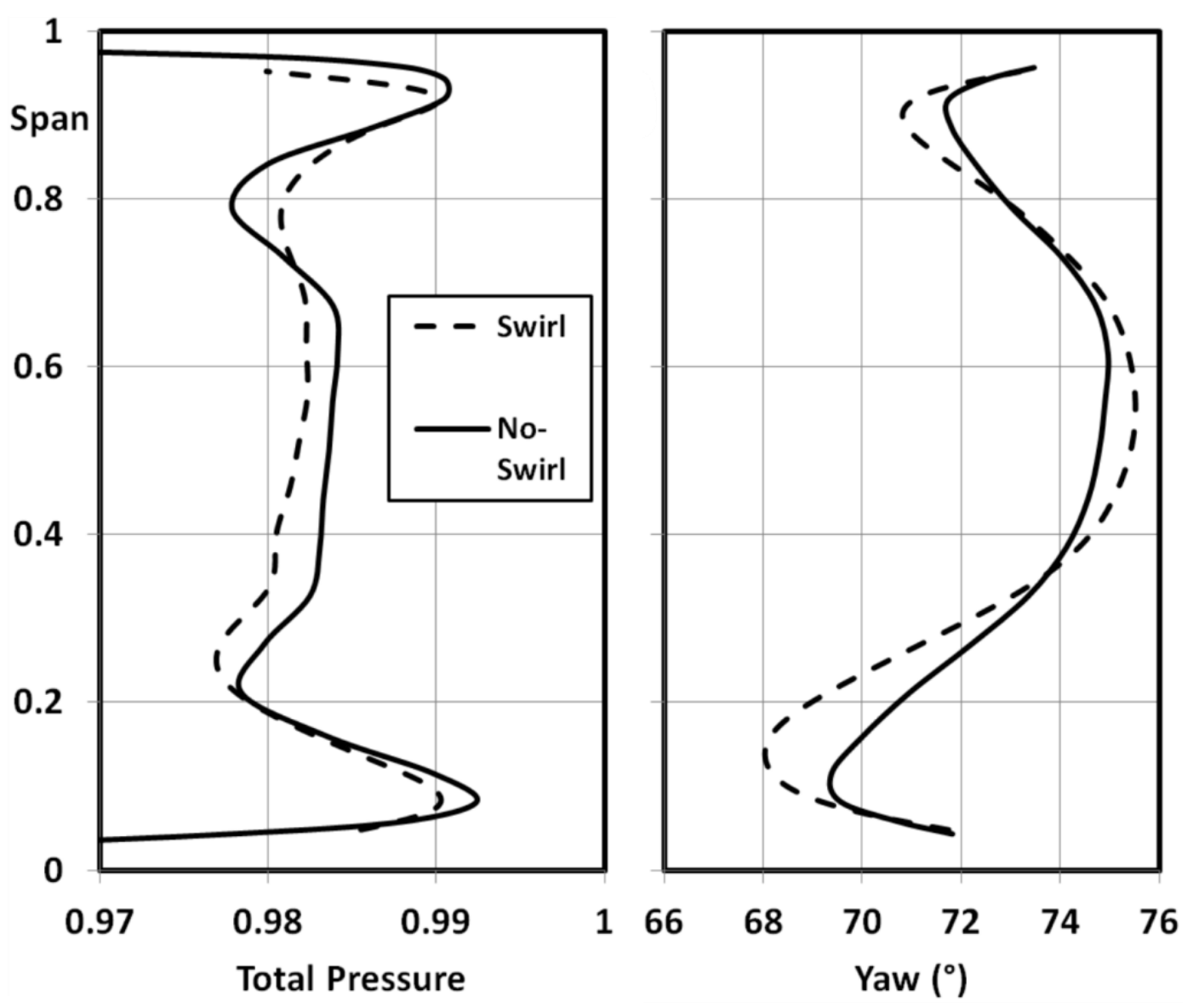

Figure 8-8: Pitchwise averaged dimensionless total pressure (left) and yaw (right) obtained from experimental traverse measurements for the swirl and no-swirl cases The effect of swirl on the aerodynamic performance thus seems to be less pronounced for the conventional vanes than for the integrated vanes. This is due to the passage-central vane of the conventional vane design that splits the swirl loss core and reduces the residual swirl. 


\subsection{Comparison of heat transfer}

In order to see the effect of combustor flow with swirl on the vanes thermal performance, infrared measurements were performed with the two swirl inlet cassettes. Figure 8-9 thus shows the measured heat transfer coefficient on the suction surfaces of the conventional and integrated vane design with inlet swirl.

a) Conventional vane with swirl unshielded
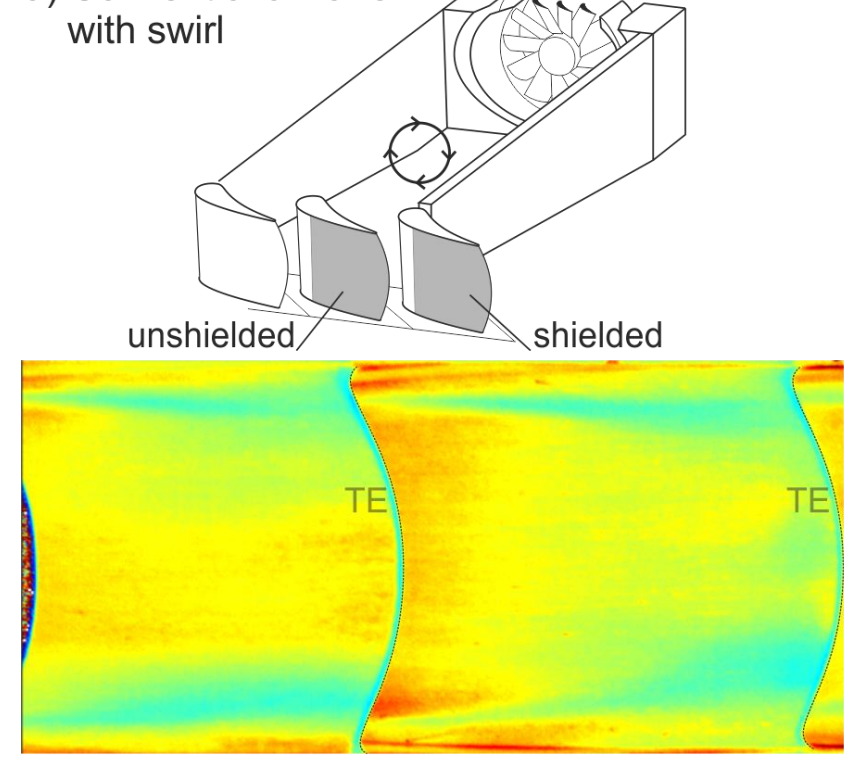

b) Integrated vane

with swirl

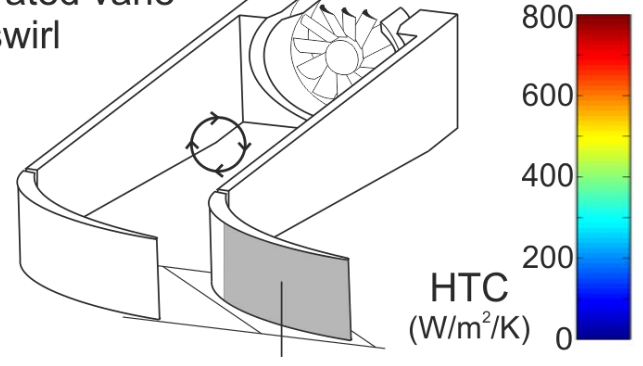

TE

Figure 8-9: Measured heat transfer coefficient on conventional vane (a) and integrated vane (b) suction surfaces with inlet swirl

For the conventional vane design it can be seen that the heat transfer coefficient distribution of the shielded vane remains very similar to the no-swirl measurements in Figure 6-1, with only slightly increased levels. The unshielded vane is however considerably more affected due to being aligned with the upstream swirler. Levels are increased by approximately $10 \%-15 \%$ and the distribution is not symmetric anymore, with increased secondary flow at the hub and decreased secondary flow at the casing due to the negative and positive upstream yaw respectively. For the integrated vane design, heat transfer coefficient levels remain significantly lower (30\%-50\%) compared to the conventional vane design. Compared 
to the no-swirl case (in Figure 6-1), levels are slightly increased likely due to increased turbulence. Furthermore, the distribution shows the slightly increased and decreased secondary flows at hub and casing respectively (caused by the negative and positive upstream yaw and its effect on vane loading), illustrated in Figure 8-2 and Figure 8-6.

\subsection{Heat transfer of integrated vane}

To obtain a better understanding of the influence of engine-realistic combustor flow on the integrated vane, thermal measurements were conducted for two scenarios with opposing swirl direction but equal swirl magnitude.

Figure 8-10 shows the measured heat transfer coefficient contours and predicted local isentropic Mach number lines on the integrated vane's suction surface. Compared to a no-swirl scenario with a horizontally symmetric heat transfer coefficient distribution (see Figure 6-1b), the swirl scenario leads to a shift in the heat transfer coefficient contour. The redistribution of the flow leads to high local isentropic Mach numbers towards the casing $(A)$ and thus high heat transfer levels in this region. Furthermore, the swirl causes negative upstream yaw at the hub (see Figure 8-1) which increases the blade loading in this region and increases the migration of low-momentum fluid up the suction surface thus causing a stronger hub secondary flow (B) (also seen in Figure 8-2b and Figure 8-6). A reversed swirl orientation leads to the opposite behaviour and the same reasoning can be applied. Good qualitative agreement between experimental measurements and numerical simulations is observed with an overestimation of peak HTC levels by approximately $20 \%$. Overall, the heat transfer coefficient values of the no-swirl, swirl and reversed swirl scenario are of comparable magnitude due to similar levels of turbulence intensity. 


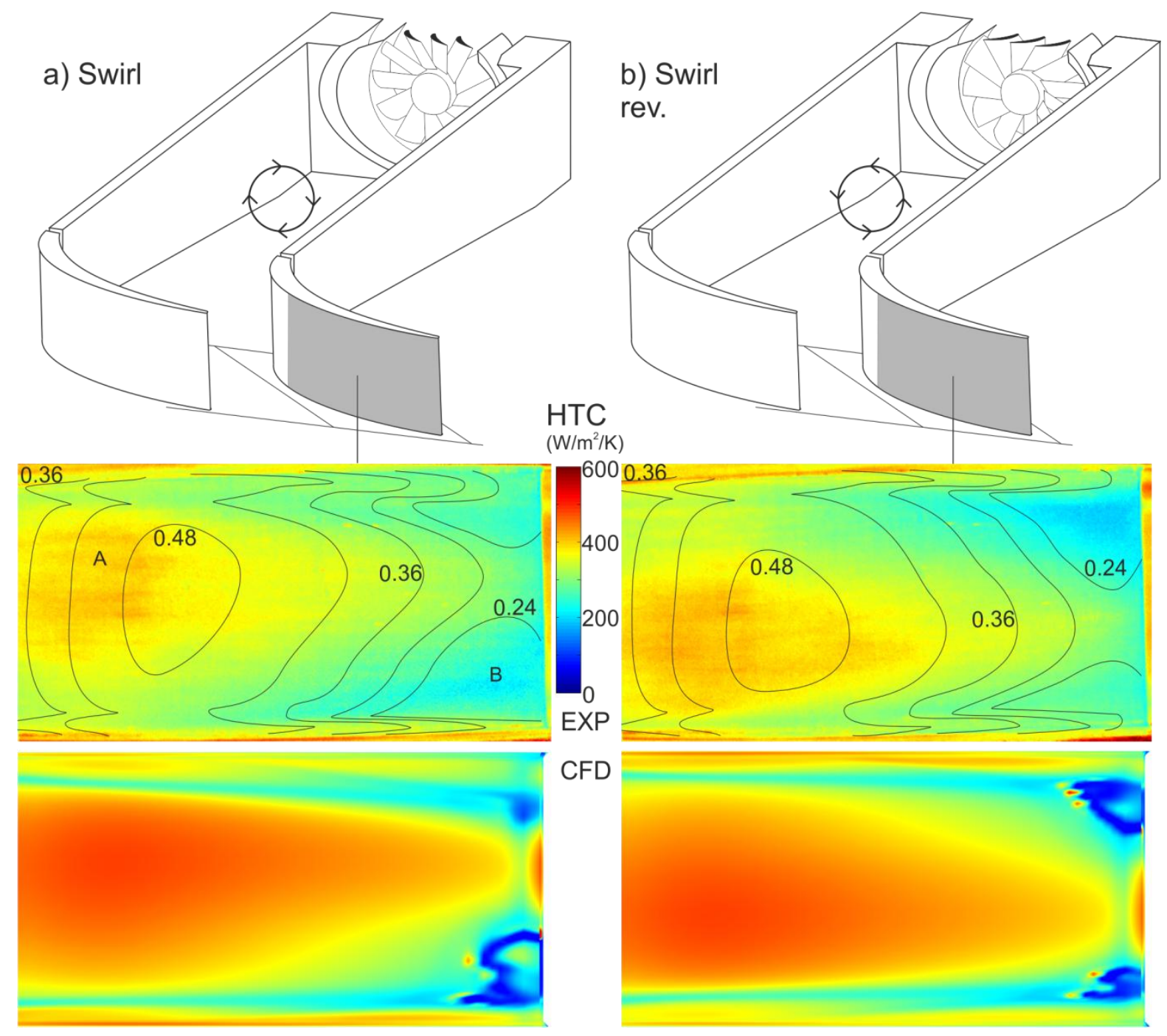

Figure 8-10: Measured HTC contour and predicted isentropic Mach number lines (top) and predicted HTC contour (bottom) on integrated vane's suction surface with swirl (left) and reversed swirl (right)

Figure 8-11 shows the measured heat transfer coefficient distributions and predicted local isentropic Mach number lines on the integrated vane's pressure surface for a swirl and a reversed swirl scenario. Compared to the horizontally symmetric heat transfer coefficient distribution seen for the no-swirl case (see Figure 6-2b) the swirl scenario leads to increased heat transfer coefficient levels towards the casing (A). This is due to the higher local isentropic Mach numbers caused by the redistribution of the flow due to the incoming swirl. The opposite effect is seen for the reversed swirl orientation and a corresponding explanation holds for the local isentropic Mach 
number levels and the heat transfer coefficient distribution. Agreement between experimental measurements and numerical simulations is qualitatively good, with slightly underpredicted HTC levels towards the hub for the swirl case.
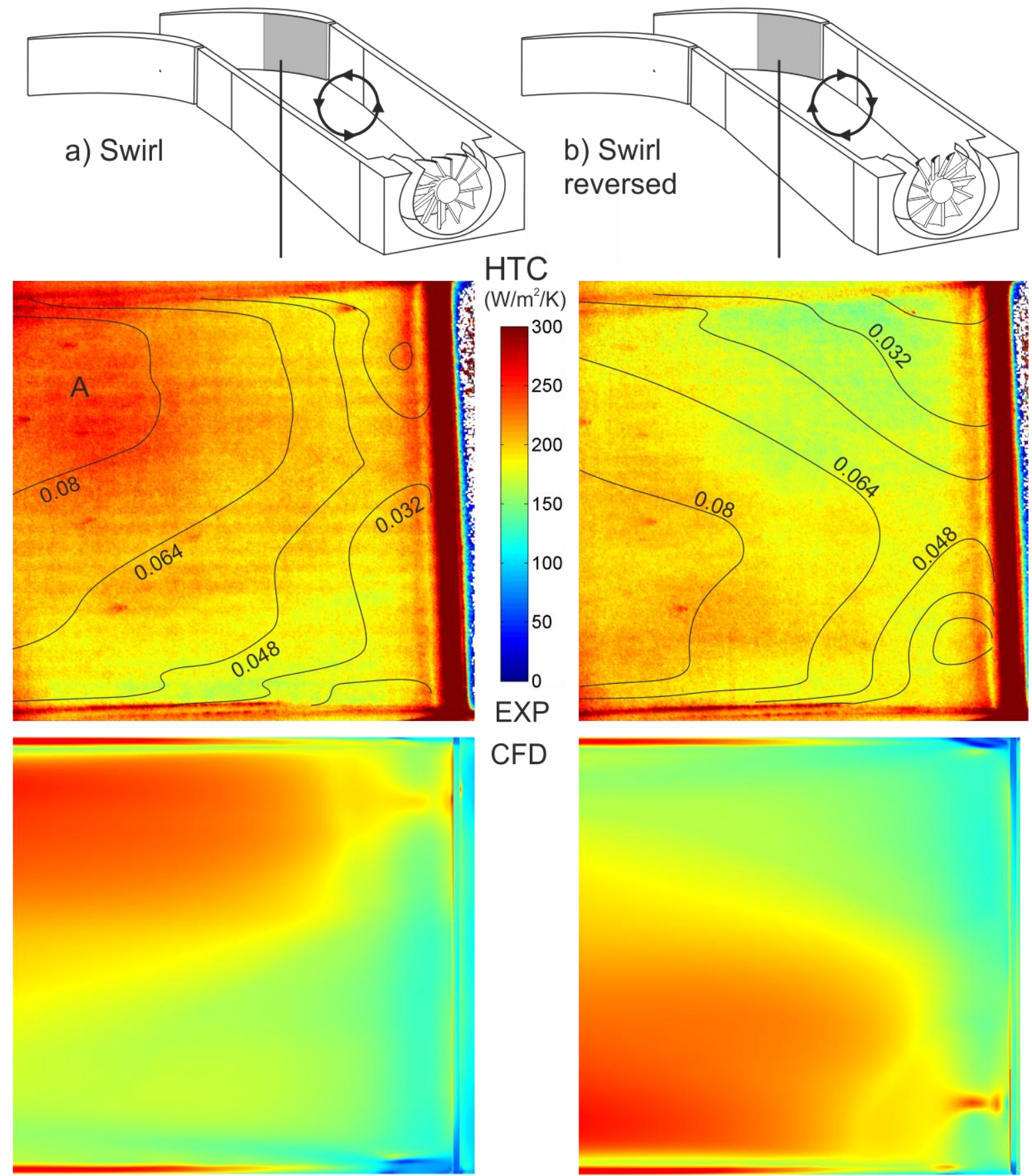

CFD

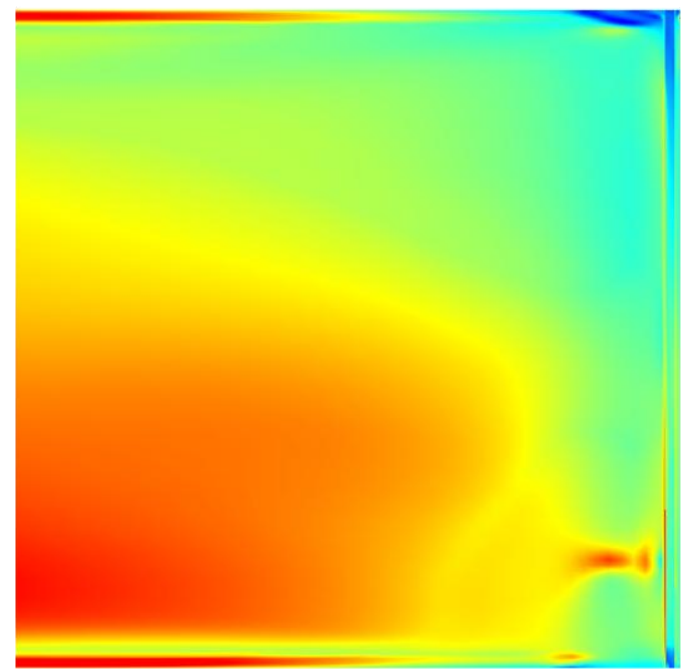

Figure 8-11: Measured HTC contour and predicted isentropic Mach number lines on integrated vane's pressure surface with swirl (left) and reversed swirl (right)

Figure 8-12 shows the measured heat transfer coefficient on the integrated vane's endwall with swirl and with reversed swirl. The differing strength of the secondary 
flow on the suction side can be seen and the distributions agree well with the measurements presented in Figure 8-10. The swirl scenario leads to approximately $20 \%$ higher HTC levels (compared to the reversed swirl scenario) on the endwall close to the suction side due to the thin boundary layer in this region (see Figure 8-2), and slightly lower HTC levels on the endwall close to the pressure side.

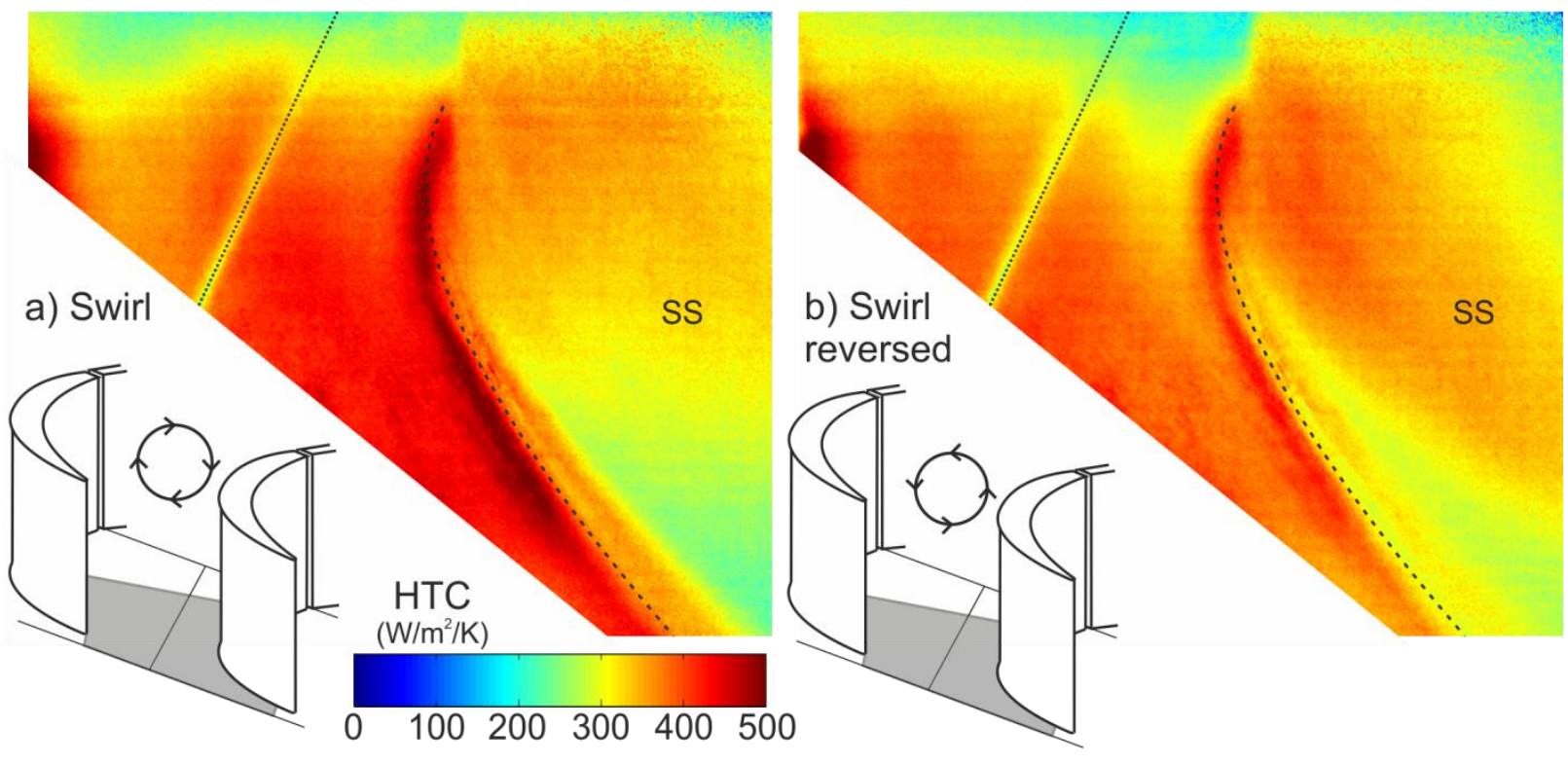

Figure 8-12: Measured HTC on integrated vane's endwall with swirl (a) and reversed swirl (b)

In conclusion it can be seen that the influence of swirl leads to only a slight, nondetrimental shift in the heat transfer coefficient distributions on the integrated vane surfaces and endwalls. The influence of the combustor swirl on the downstream aerodynamics is, however, more significant. While the wakes are only slightly distorted, the turning is considerably altered. Subsequent investigations should thus focus on the influence of the flow redistribution on the rotor, and determine if a threedimensional integrated vane design could be beneficial for the stage performance. Furthermore it is assumed that the swirl generators can potentially lead to additional unsteadiness due to the formation of a precessing vortex core. Although not examined in this thesis, future studies should address the implications of this 
unsteadiness on the integrated vanes full-stage aerothermal performance and cooling.

\subsection{Stage performance of conventional and integrated vane}

The experimental traverse measurements of Figure 8-2, Figure 8-3 and Figure 8-5 showed significant variations in turning downstream of the integrated vane due to swirl, and a convection of the swirl core through the vane passage (see also Figure 8-6). Full stage simulations were thus performed for conventional and integrated vane domains with the swirl inlet (see Figure 8-1a) to compare the effect of swirl on the downstream rotor for both designs. Figure 8-13 shows the lost efficiency through conventional and integrated vane domain averaged over one cycle for the no swirl case (solid lines, previously shown in Figure 7-3) and the swirl case (dotted lines).

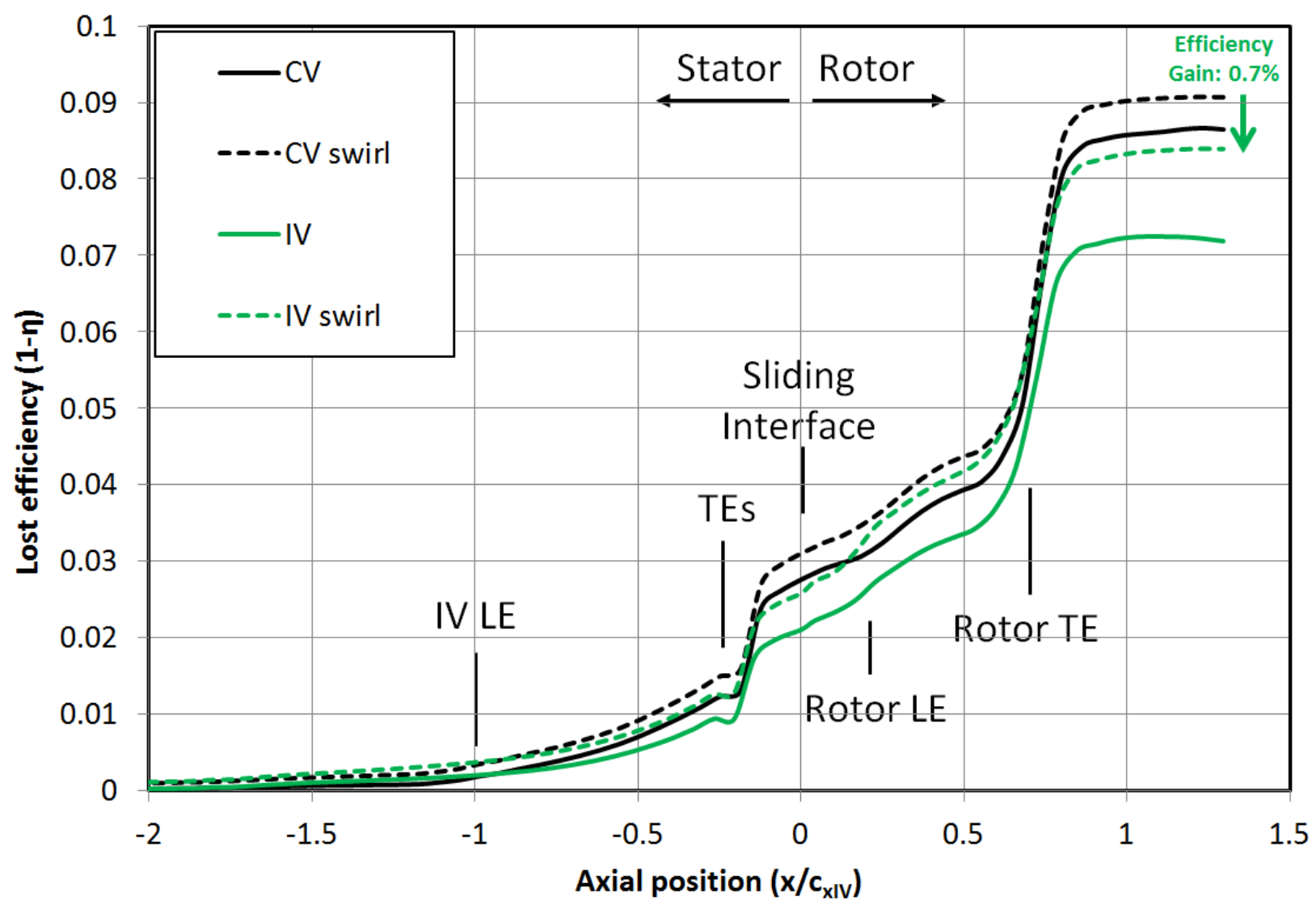

Figure 8-13: Influence of swirl on stage efficiency loss for conventional and integrated vanes 
It can be seen that at the sliding interface the conventional vane's stage efficiency decreased by $0.3 \%$ due to swirl, while the integrated vane's stage efficiency decreased by $0.5 \%$ due to swirl. At the exit of the domain, the influence of swirl resulted in a stage efficiency reduction of $0.4 \%$ for the conventional vane, and of $1.2 \%$ for the integrated vane. This shows that most of the loss for the conventional vane design occurs in the stator $(75 \%)$ and the rotor is not significantly affected. For the integrated vane design on the other hand, the stage efficiency loss in the stator 'only' accounts for $40 \%$ while a further $60 \%$ is generated in the rotor. Swirl thus has a stronger effect on the integrated vane design. Nonetheless, the integrated vane design still shows a stage efficiency advantage of $0.7 \%$ over the conventional vane design (down from 1.5\% in Figure 7-3).

In order to obtain a better understanding of the reasons for the differences in the effect of swirl on the stage performance of conventional and integrated vane, a detailed look at the entropy inside of the stage is presented. Figure 8-14 shows the instantaneous entropy function at midspan, upstream and downstream of the rotor for the conventional vane stage simulation with swirl. It can be seen that the swirl core is 'split' by the unshielded conventional vane, convected along pressure and suction side, and still visible downstream of the vane. As a result, the unshielded vane's wake is now slightly stronger than the shielded vane's wake (opposite to the no-swirl case in Figure 7-5). The wakes are similarly distributed across several rotor passages and convected through the rotor. The downstream distribution of the entropy function with swirl looks relatively similar to the no-swirl case (see Figure 7-9), suggesting that the influence of swirl on the rotor is of minor strength. 
a) At midspan

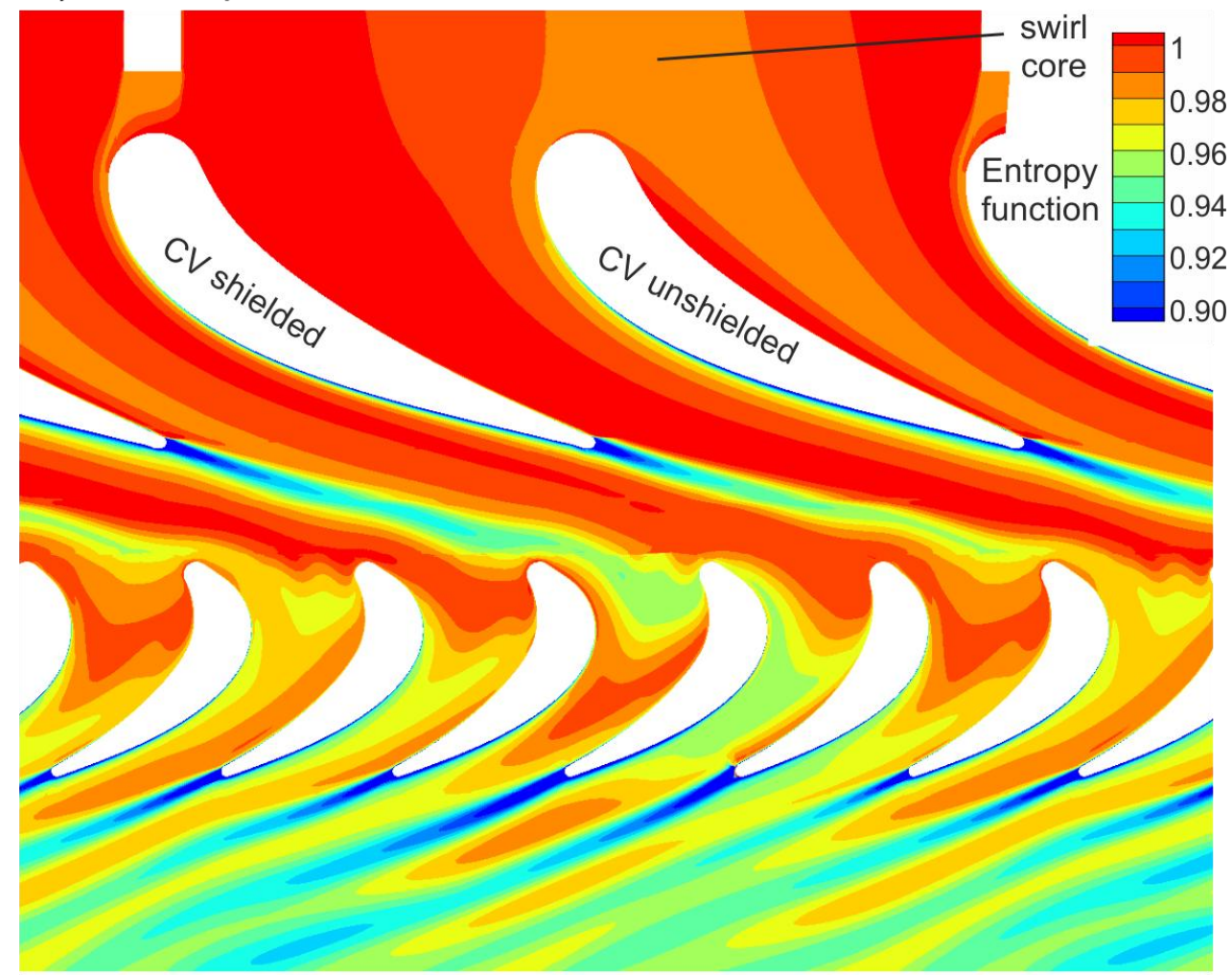

b) At sliding interface

wake of unshielded CV

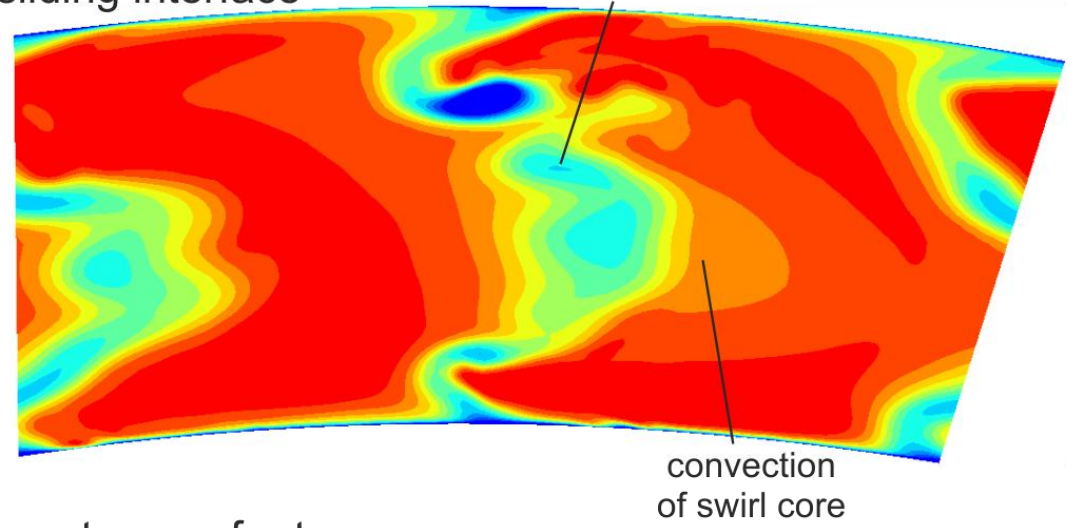

c) Downstream of rotor

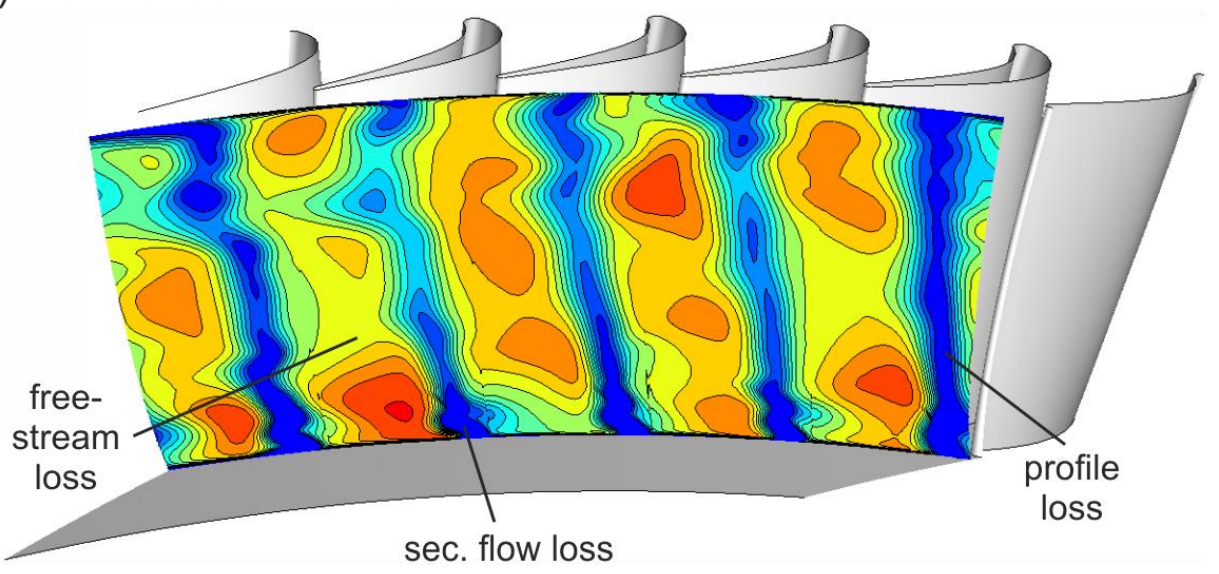

Figure 8-14: Instantaneous entropy function at midspan (a), and axial cut upstream (b) downstream (c) of rotor for conventional vane stage simulation with swirl 
To obtain a better understanding of the swirl's limited effect on the rotor of the conventional vane stage it is important to understand the flowfield at the stator-rotor interface, which is mainly described by the total pressure and turning distributions. Figure 8-14 illustrated the effect of swirl on the total pressure within the stage with help of the entropy function. Figure 8-15 on the other hand shows the time- and pitchwise averaged turning at the sliding interface (i.e. between stator and rotor) for conventional (black) and integrated (green) vane design for a no-swirl (solid line) and a swirl (dotted line) case.

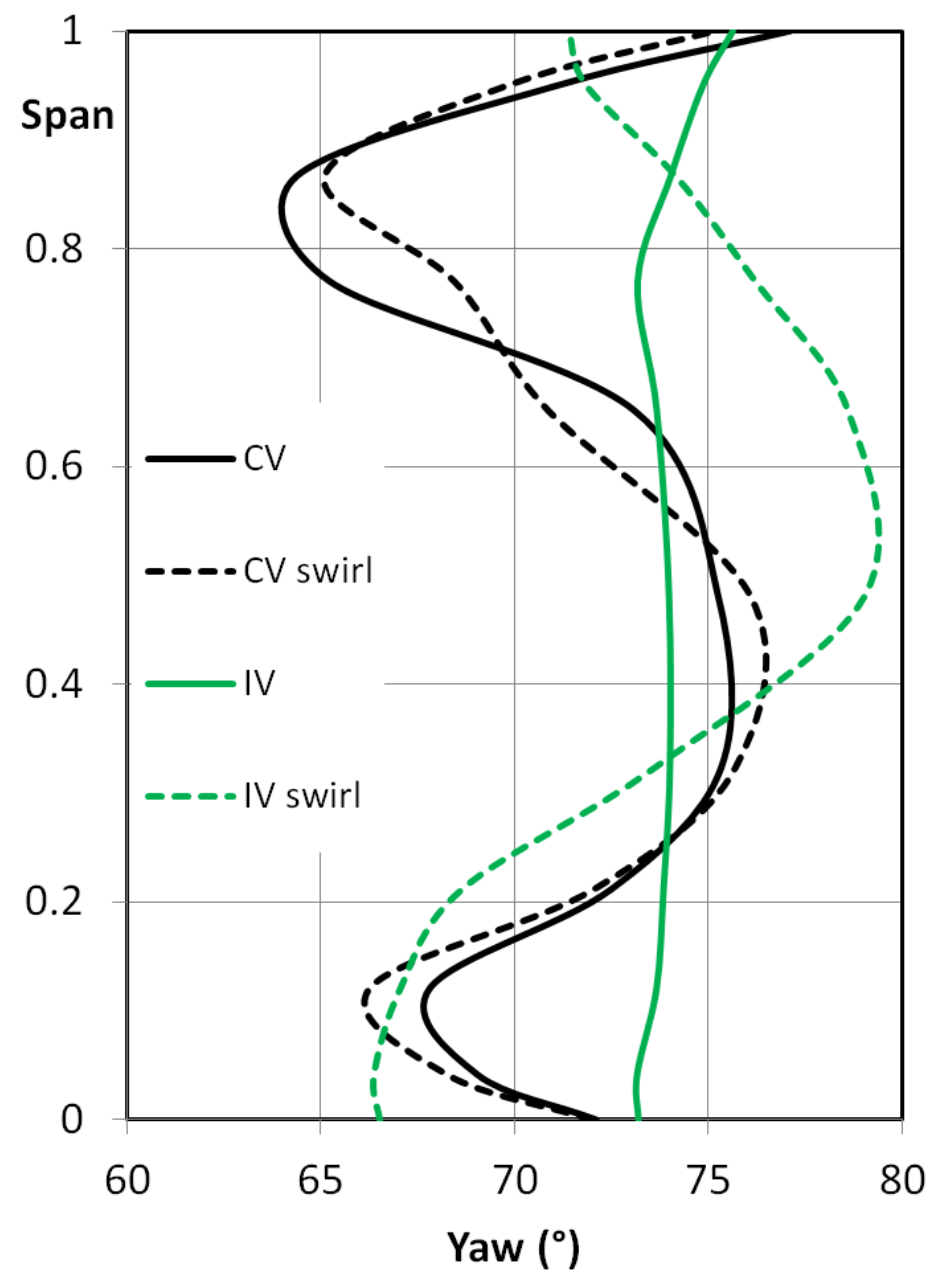

Figure 8-15: Time- and pitchwise-averaged turning at the sliding interface for a noswirl (solid line) and swirl (dotted line) case

For the conventional vane design, it can be seen that turning is highest at midspan (due to the vane compound lean with increased metal exit angle at midspan), and 
also high at the endwalls due to the overturning of the low-momentum fluid (i.e. endwall boundary layer). It is evident that inlet swirl does not significantly change the turning distribution downstream of the conventional vanes, with only slightly decreased $\left(-2^{\circ}\right)$ and increased $\left(+2^{\circ}\right)$ turning at $10 \%$ and $80 \%$ of span respectively. The trend is plausible since the upstream traverse measurements of Figure 8-1 indicated positive and negative yaw in the top and bottom half respectively for the swirl case.

A very different behaviour can be observed for the integrated vane. For a no-swirl case, pitchwise averaged turning is nearly constant across the span due to the effectively two-dimensional profile of the integrated vane. Turning is however significantly altered with inlet swirl. Due to the lack of a passage-central vane, the swirl core is simply transferred through the vane passage, leading to underturned and overturned flow (up to $\pm 6^{\circ}$ ) in the bottom and top half of the domain respectively (in-line with the trend of the upstream turning distribution in Figure 8-1).

The spanwise non-uniformity in turning downstream of the integrated vane due to swirl results in varying incidence at the rotor inlet. Figure 8-16 thus shows the instantaneous streamtraces on the integrated vane rotor leading edges, pressure surfaces and hub endwall for a no-swirl and a swirl case. For the no-swirl case it can be seen that the stagnation lines on the rotor leading edges are nearly linear. The streamtraces on the pressure (and the visible part of the suction) surface follow a meridional direction with a low radial velocity component. This is due to the very uniform rotor-upstream pitchwise-averaged turning. The endwall streamtraces illustrate a wide variety of rotor incidence across the different rotor passages due to a considerable turning non-uniformity in pitchwise direction (also see Figure 7-8). The horse-shoe vortex system thus varies significantly between the rotor passages. 
a) IV rotor - no-swirl
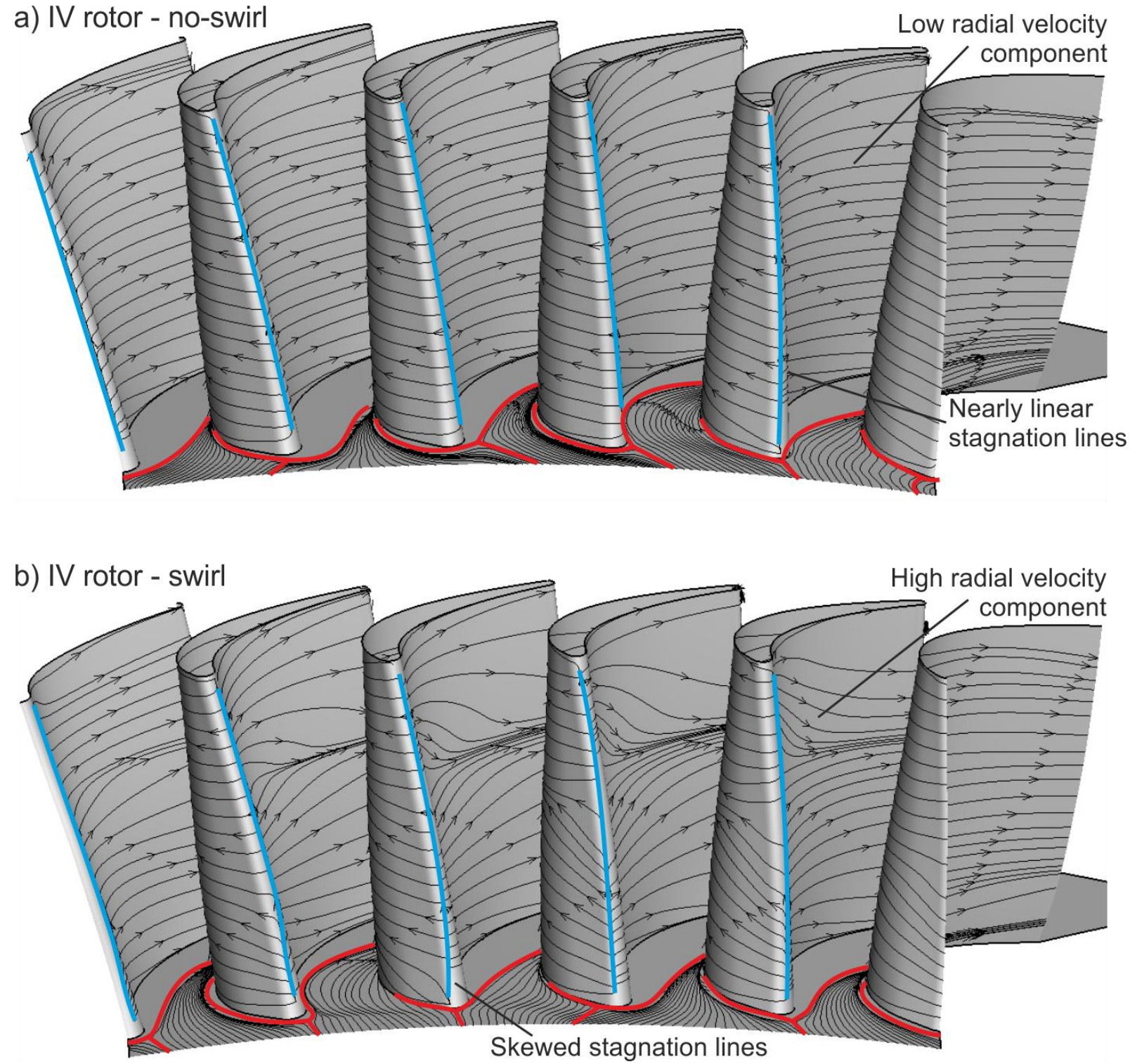

Figure 8-16: Instantaneous streamtraces on integrated vane rotor for a no-swirl (a) and a swirl (b) case

For the swirl case, it can be seen that at the endwall the rotor incidence angles are shifted more towards negative levels due to the overall lower turning in this region shown in Figure 8-15. The horse-shoe vortex system remains significantly different between the rotor passages. It is moreover visible that the stagnation lines on the rotor leading edges are skewed due to the spanwise variation in turning upstream of the rotor and the corresponding variance in rotor incidence. This also alters the loading distribution across the rotors span. As a result of this static pressure redistribution, the streamtraces on the rotor pressure surfaces show considerable 
radial velocity components, visibly in contrast to the no-swirl case. The rotor performance of the integrated vane design is thus clearly influenced by the inlet swirl and a better understanding can be obtained by looking at the entropy function distributions as done for Figure 8-14.

Figure 8-17 shows the instantaneous entropy function at $33 \%$ of span, upstream and downstream of the rotor for the integrated vane stage simulation with swirl. It can be seen that the swirl core is convected through the vane passage (also shown in Figure $8-2 b$ and Figure $8-6 a)$. It is clearly visible at the sliding interface, having radially convected closer to the hub due to the radial pressure gradient of the annular design (with higher pressures towards the casing than towards the hub). It passes through the rotor (see $\left.\mathrm{sc}_{\mathrm{i}}-\mathrm{sc}_{\mathrm{iii}}\right)$ and reduces levels of the entropy function downstream, albeit hardly distinguishable. The integrated vane wake is more skewed than in the no-swirl case (nearly radial shape in Figure $7-5 b$ ) due to the spanwise variation in turning (see Figure 8-3b). It thus spans a larger circumferential distance and becomes increasingly distorted when approaching the rotors potential field. It is thus distributed across a larger number of stator passages compared to the no-swirl case and its reduction of entropy function levels can still be seen downstream of the rotor (slightly less distinguishable than in Figure 7-9). The pronounced secondary flow hub loss cores can also be seen (downstream of passage 5), just as in Figure 7-9. Overall, levels of the entropy function are noticeably lower than for the no-swirl case. A slight increase in profile loss, and lower and less uniform levels of the entropy function in the freestream flow compared to the no-swirl case (as indicated by ' + ' in Figure 7-9) is evident. This is caused by the increased rotor incidence and respective penalty on rotor performance. 
a) At $33 \%$ span

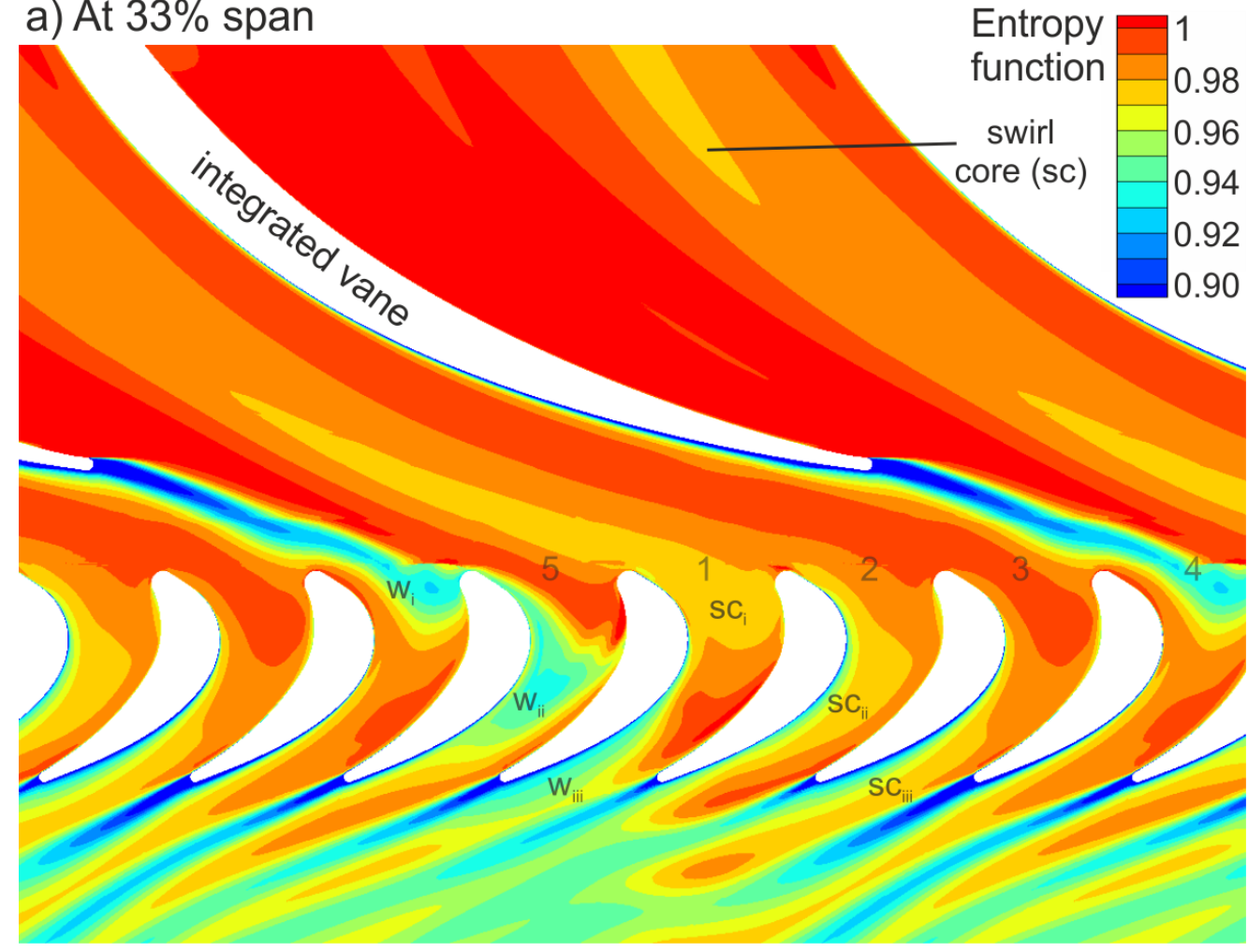

b) At sliding interface

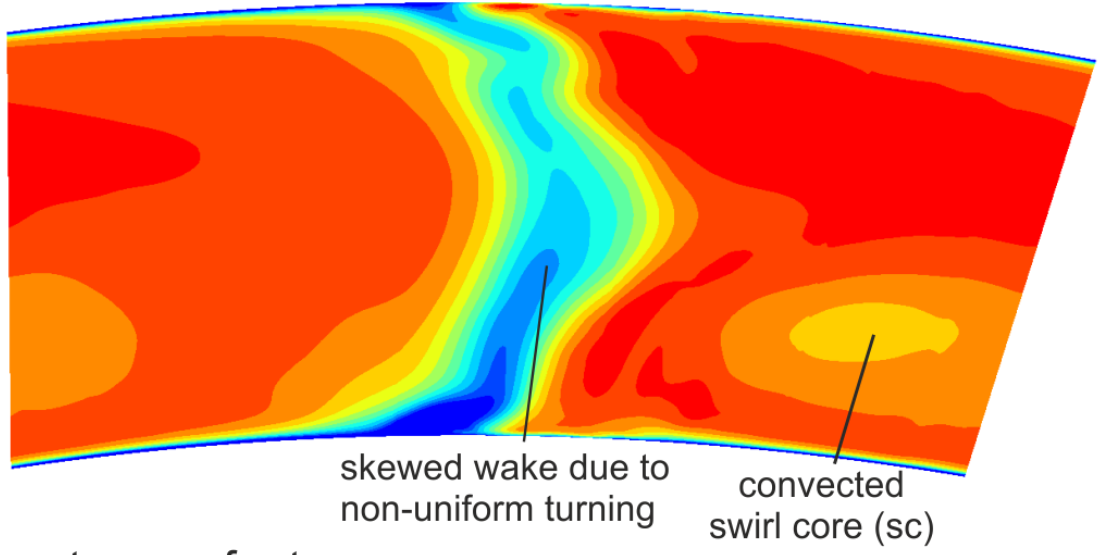

c) Downstream of rotor

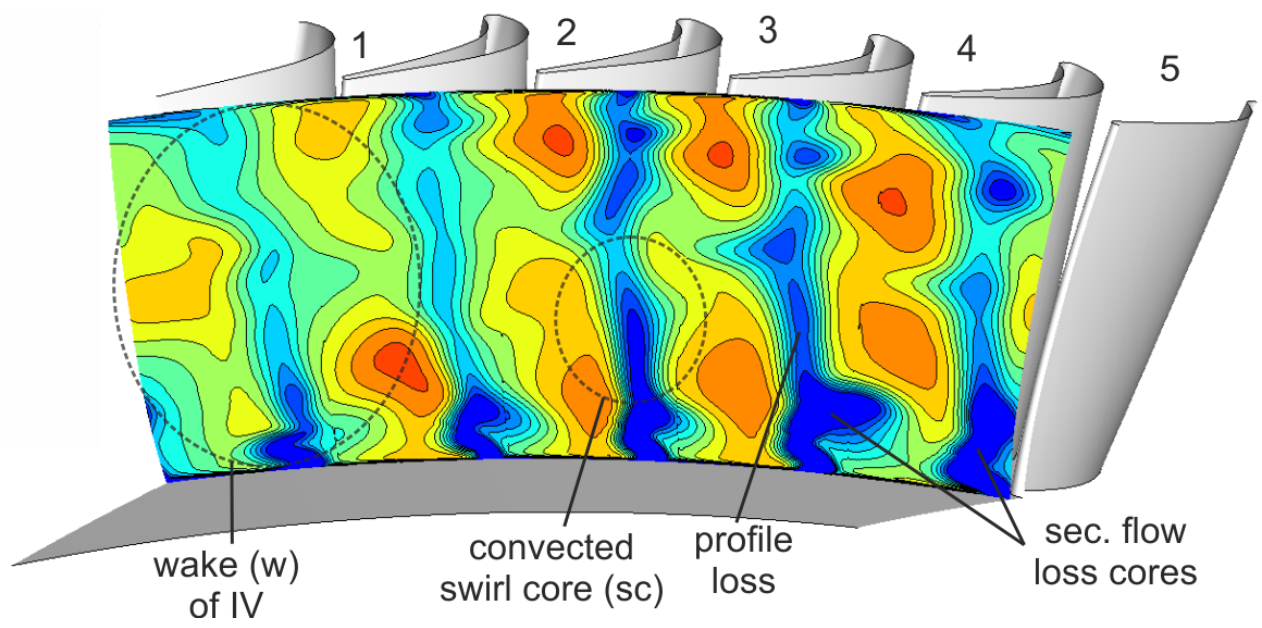

Figure 8-17: Instantaneous entropy function at 33\% span (a), and axial cut upstream (b) and downstream (c) of rotor for integrated vane stage simulation with swirl 
Time- and pitchwise averaging the entropy function distributions at the sliding interface (rotor inlet) and exit of the domain (rotor outlet) for the swirl case, as shown in Figure 8-18, allows for a more quantitative comparison of the stage performance between conventional and integrated vane design.

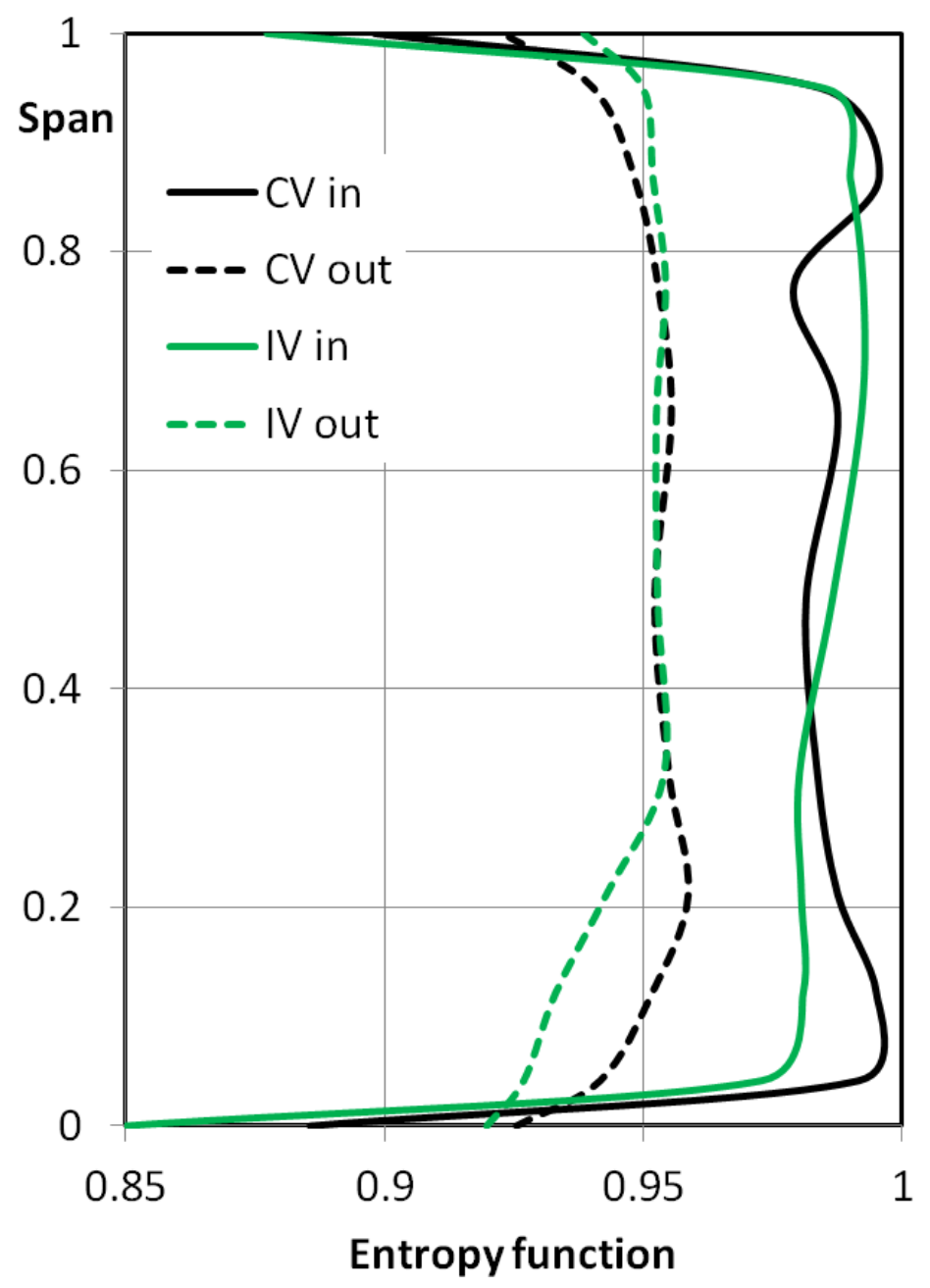

Figure 8-18: Time- and pitchwise-averaged entropy function at rotor inlet and outlet for the swirl case

It can be seen that at the sliding interface the integrated vane (green) has a slightly higher loss at the endwalls due to the thicker boundary layers (caused by removal of horse-shoe vortex system). Moreover loss is higher between $0 \%-35 \%$ as a result of the pronounced integrated vane hub loss core (caused by reduced aspect ratio and increased loading of integrated vane compared to conventional vane). Downstream of the rotor, loss is higher and lower for the integrated vane between $0 \%-30 \%$ and 
$80 \%-100 \%$ respectively. The former is caused by the strong rotor hub secondary flow and negative rotor incidence (due to underturned flow upstream of the integrated vane's rotor) in this region. The latter is caused by the negative incidence (due to underturned flow upstream of the conventional vane's rotor) in this region.

Combining all effects of the swirl (skewed wake, swirl core, non-uniform turning) on the stage results in its reduced performance by $1.2 \%$ compared to the no-swirl case. It nonetheless outperforms the conventional vane design by $0.7 \%$ stage efficiency (see Figure 8-13). It should however be remembered that the swirlers chosen for this study (generic design) have a stronger swirl strength than is common in industrial practice so that the actual impact would be less drastic. In order to reduce the effect of swirl on the integrated vane's full stage performance, it is suggested to use vane profiling to minimize non-uniformities of turning downstream of the integrated vane.

\subsection{Integrated vane profiling for swirl}

The initial objective of the integrated vane development was to match the flow turning of conventional vanes whilst ideally reducing aerodynamic loss. This resulted in a simple two dimensional design, omitting vane profiling like sweep and lean. In the course of the study it was however shown that the residual swirl at the inlet of the vanes, caused by engine realistic combustor geometry, leads to a significant nonuniformity of turning upstream and downstream of the integrated vanes. The lack of a duct-central vane, as with nozzle guide vanes, resulted in a stronger influence of swirl on stage performance for the integrated vane concept (see Figure 8-13). This chapter thus aims to investigate if three-dimensional integrated vane profiling can help to mitigate the effect of inlet swirl on the aerodynamic stage performance. 
In order to come up with a new three-dimensional integrated vane design it is necessary to recall the aerodynamic traverse measurements with swirl of Figure 8-1 and Figure 8-3. Since swirl increases turning in the top half of the domain and reduces it in the bottom half, the new vane design should feature a reduced vane exit metal angle at the casing and an increased one at the hub. Figure 8-19 shows the final geometry of the three-dimensional integrated vane design. It can be seen that the design only features vane lean (same axial position of trailing edge along span) and keeps a constant trailing edge thickness. An iterative design approach was used to maintain averaged downstream flow turning while achieving a more homogenous spanwise yaw distribution.
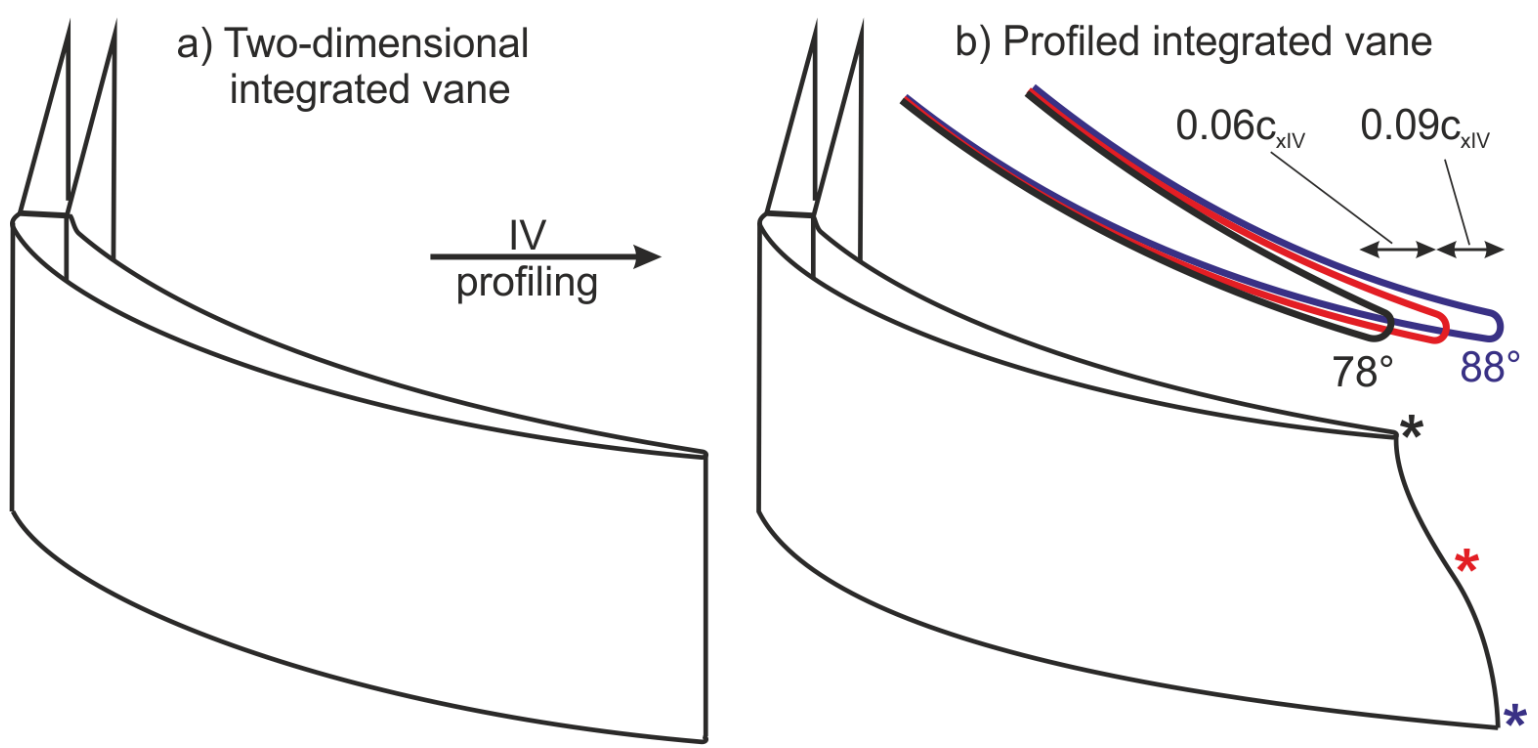

Figure 8-19: Schematic of three-dimensional vane profiling with 'original' twodimensional integrated (a) and 'new' profiled integrated vane (b)

Figure 8-20 thus shows the turning distribution downstream of the two- and threedimensional integrated vane, and also a comparison of pitchwise averaged yaw obtained from linear CFD simulations. 

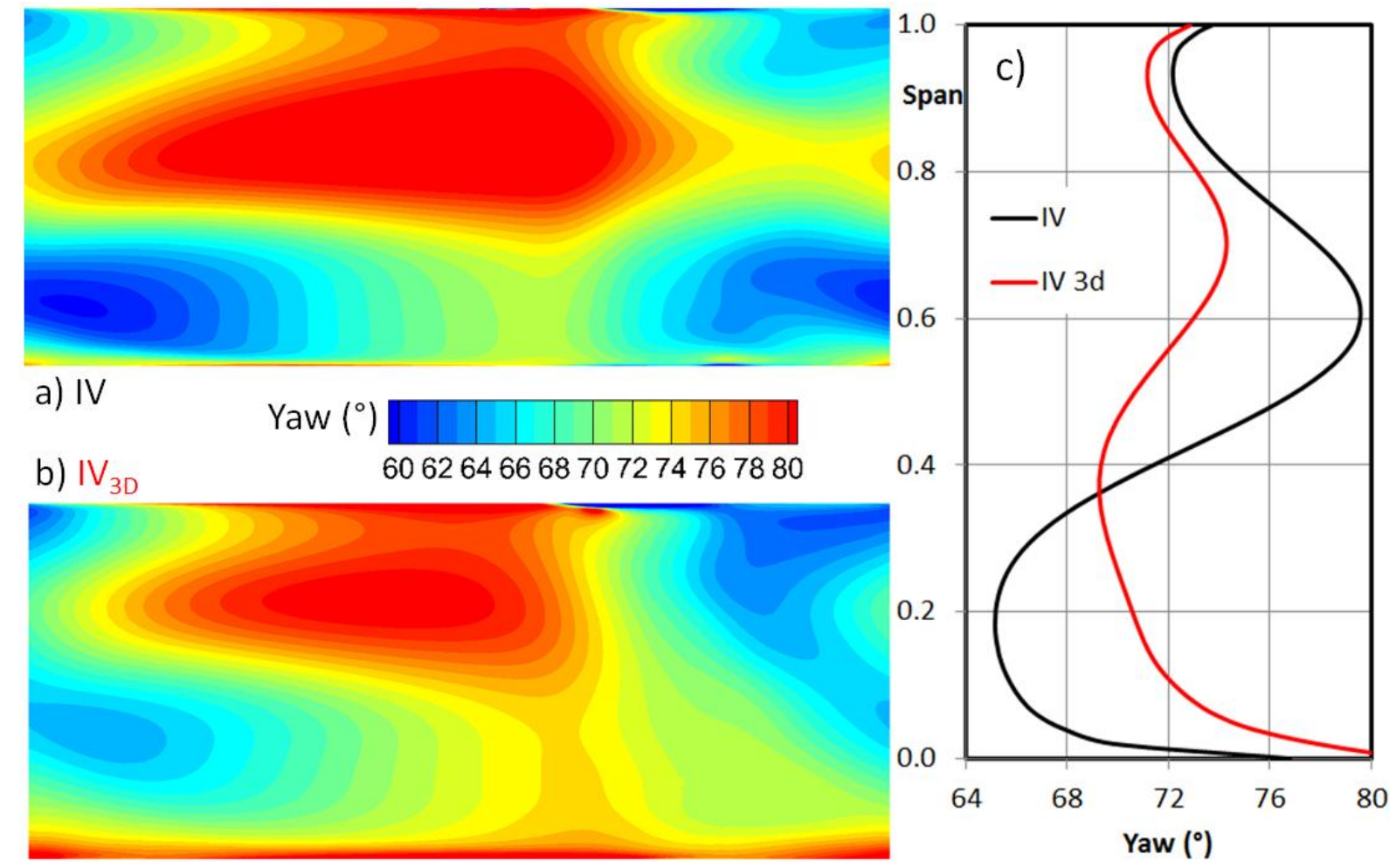

Figure 8-20: Predicted turning downstream of two- (a) and three- (b) dimensional integrated vane, and pitchwise averaged yaw (c) for steady linear simulation

It can be seen that the turning is reduced in the top half, and increased in the bottom half of the domain for the three-dimensional integrated vane. The graph of pitchwise averaged yaw confirms the trends seen in the turning distributions and shows a significantly more homogenous turning in spanwise direction downstream of the three-dimensional integrated vane, except for the increased overturning at the hub endwall. While average turning stays nearly constant at $72^{\circ}$, deviations are reduced from $\pm 7^{\circ}$ to $\pm 2.5^{\circ}$ (excluding the endwall region). Continuing the iterative design approach would inevitably lead to an even more homogeneous downstream turning, but the given design shall suffice for proof of the effectiveness of the integrated vane profiling.

Figure 8-21 shows the dimensionless total pressure distribution downstream of the two- and three-dimensional integrated vane and a pitchwise averaged graph of spanwise total pressure. It can be seen that the wake of the three-dimensional 
integrated vane is slightly more skewed and that the residual swirl core remains closer to the midspan. The graph of Figure 8-21c shows only a slight decrease in total pressure downstream of the three-dimensional integrated vane close to the hub endwall, caused by an increased strength of the hub loss core (due to the increased vane turning towards the hub and thus stronger secondary flow). Total pressure is higher towards the casing due to a decreased casing loss core as a result of the reduced vane turning at the casing. The effect on total pressure is thus nondetrimental.
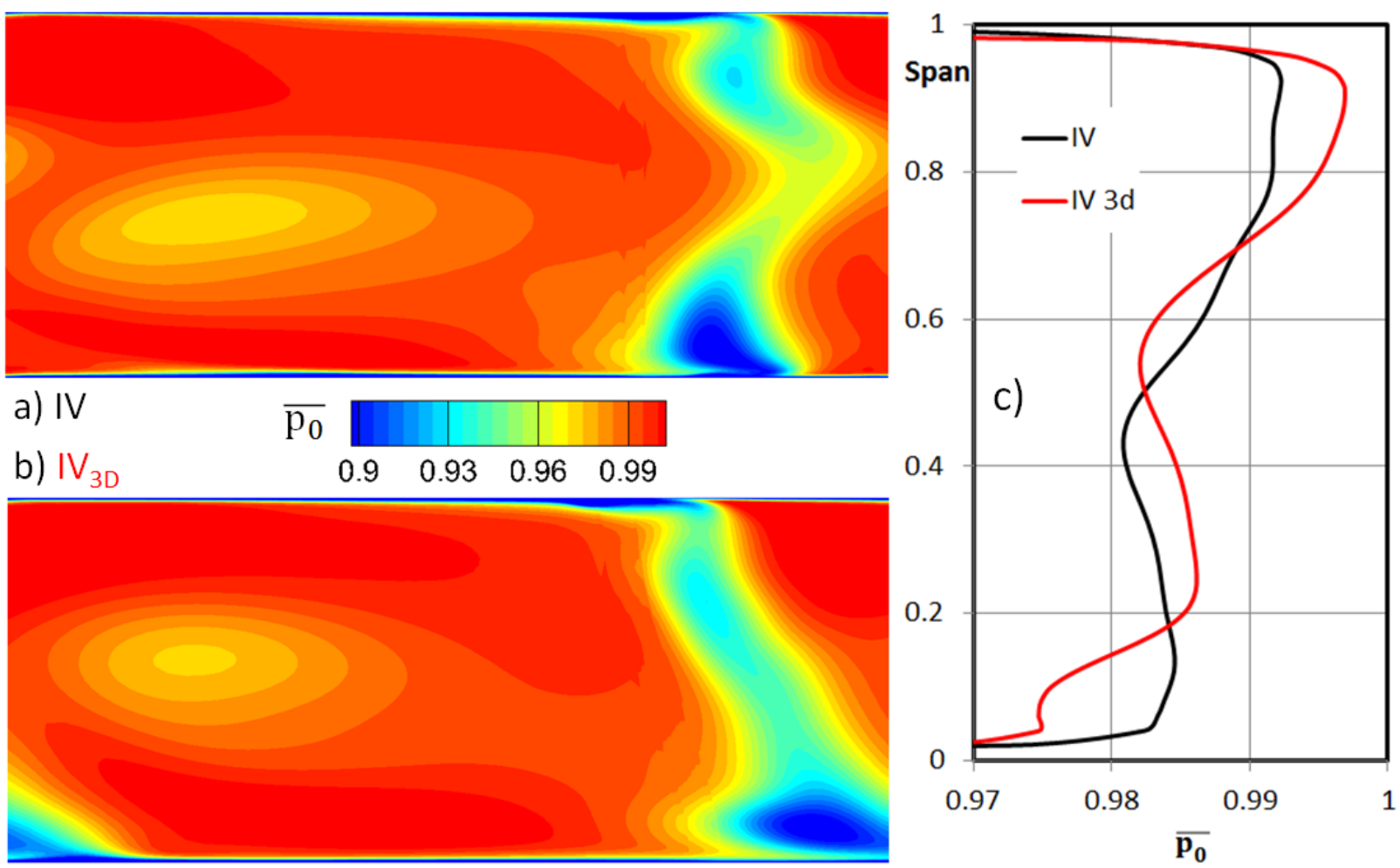

Figure 8-21: Predicted dimensionless total pressure distribution downstream of two(a) and three- (b) dimensional integrated vane, and pitchwise averaged dimensionless total pressure (c) for steady linear simulation

Figure 8-22 shows the heat transfer coefficient distribution on the two- and threedimensional integrated vane suction surface. The slightly increased hub secondary flow for the three-dimensional integrated vane can be seen. It can nonetheless be 
concluded that the vane profiling only has a negligible effect on the integrated vane's heat transfer distribution.

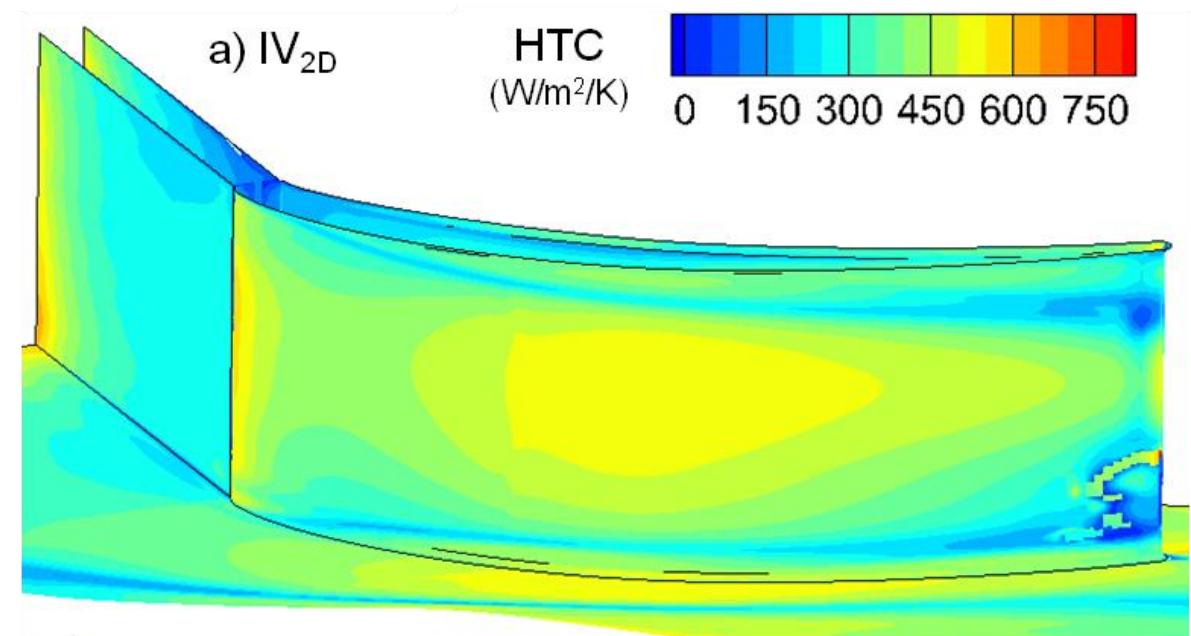

b) $I V_{3 D}$

Figure 8-22: Predicted heat transfer coefficient distribution on two- (a) and three- (b) dimensional integrated vane suction surface for steady linear simulation

Figure 8-23 shows the effect of the integrated vane profiling on the efficiency loss, obtained from full stage simulations with swirl. It can be seen that the profiled integrated vane successfully reduces the efficiency loss downstream of the vane's trailing edge. At the sliding interface the lost efficiency is reduced by $0.46 \%$. The distributions stay nearly parallel within the rotor. The losses of the profiled integrated vane however increase slightly more at the outlet of the rotor. The gains obtained within the stator are thus partially offset by a reduced rotor performance and the stage efficiency is increased by a total of $0.23 \%$ at the exit of the domain. 


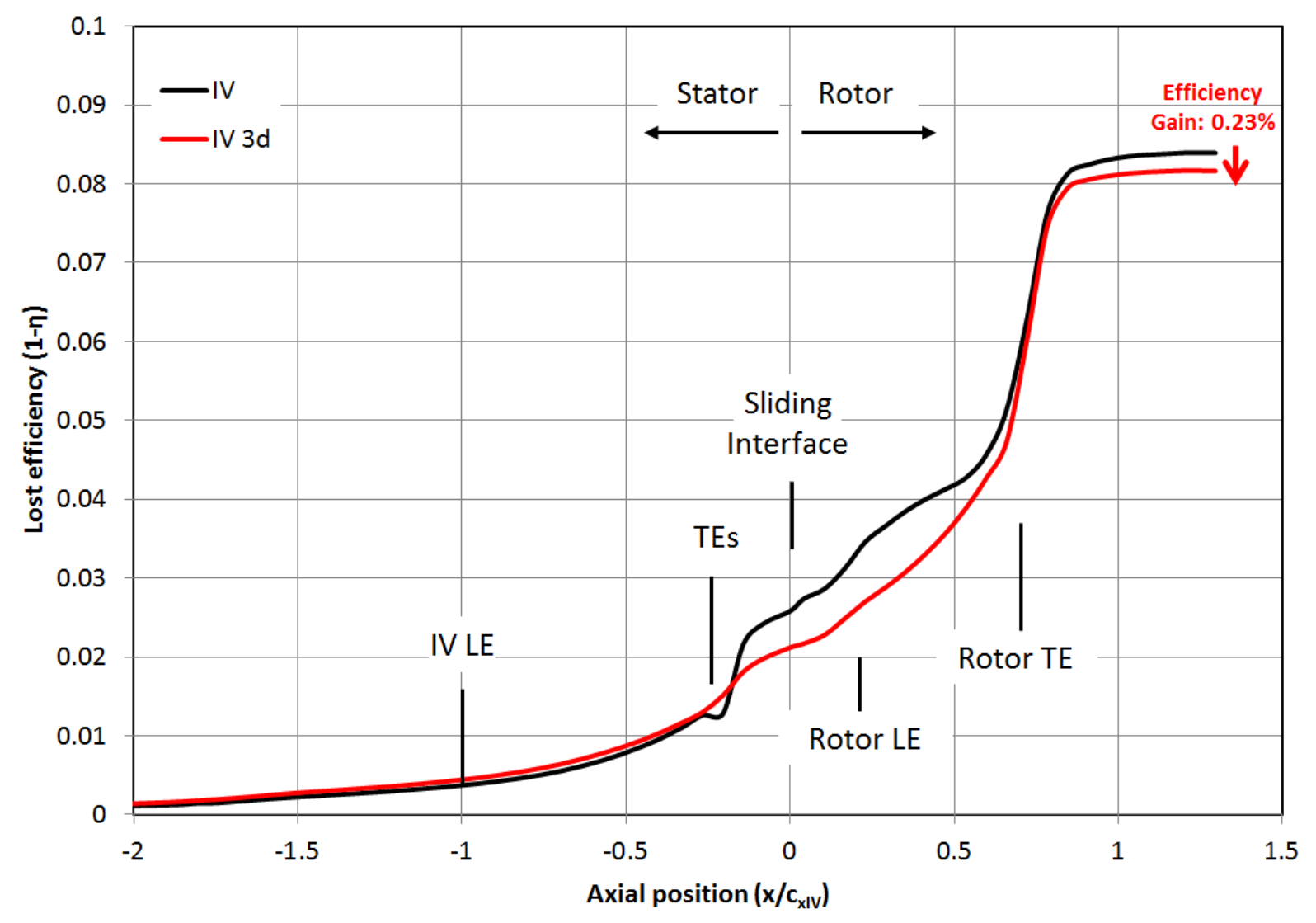

Figure 8-23: Predicted effect of integrated vane profiling on stage efficiency loss

To get a better understanding of the loss generation within the stage, distributions of the entropy function will be used. Figure 8-24 shows the instantaneous entropy function at $33 \%$ of span, upstream and downstream of the rotor for the profiled integrated vane full stage simulation with swirl. It can be seen that the wake of the profiled integrated vane spans a larger circumferential distance due to the decreased and increased vane turning at the casing and hub respectively. The low-pressure core stays closer to the midspan (also seen in Figure 8-21) due to an altered radial static pressure field (increased and reduced static pressure at hub and casing respectively) caused by the changed vane loading across the span. The wake and swirl core are similarly convected through the rotor as for the 'normal' integrated vane. A look at the entropy function distribution downstream of the rotor shows that the profiled integrated vane's wake (downstream of the rotor passages 3-4) is slightly 
less apparent. This is because the wake is distributed across more rotor passages due to spanning an increased circumferential distance at the inlet of the rotor.

a) At $33 \%$ span

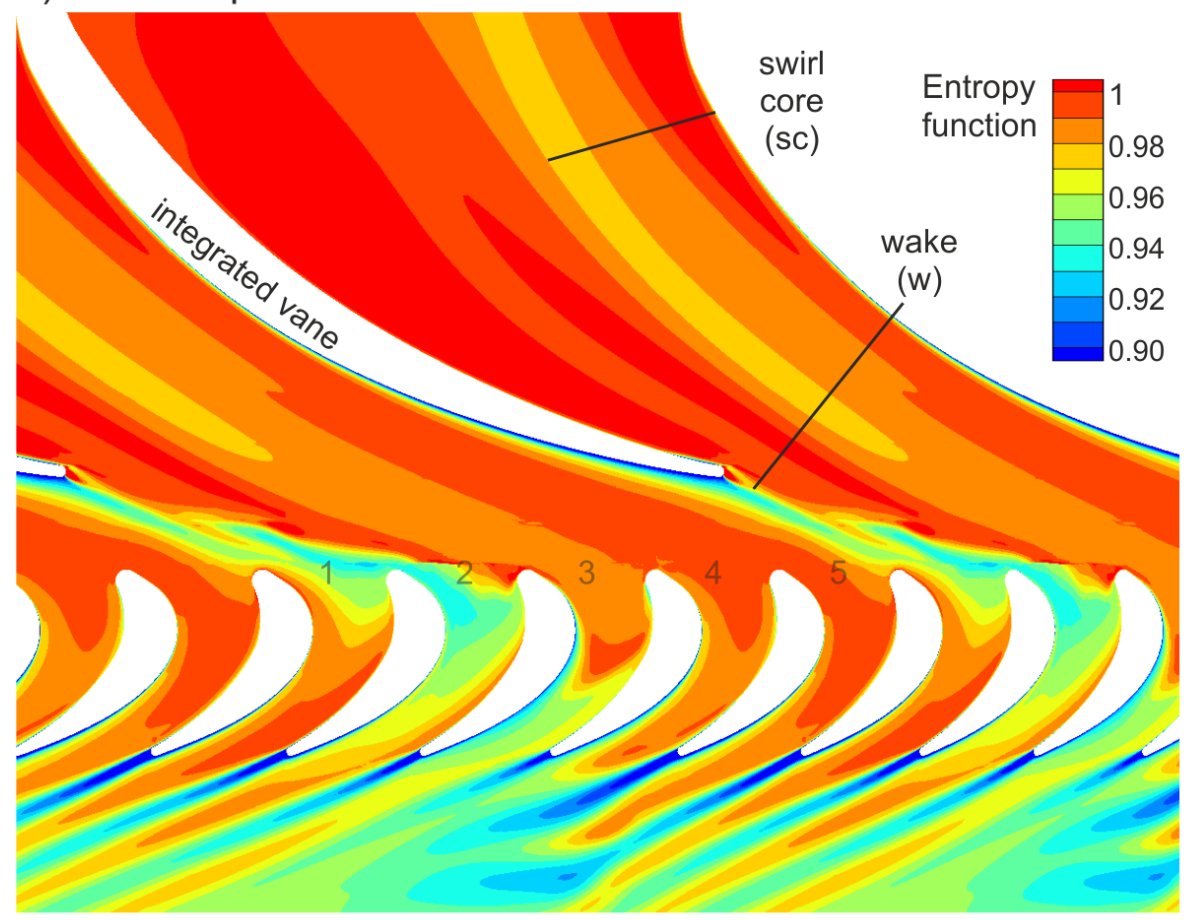

b) At sliding interface convected swirl core (sc)

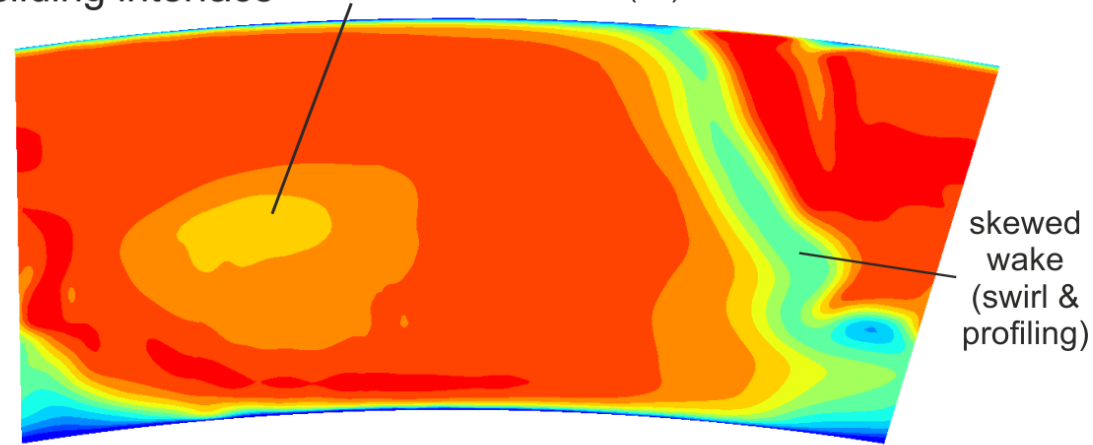

c) Downstream of rotor

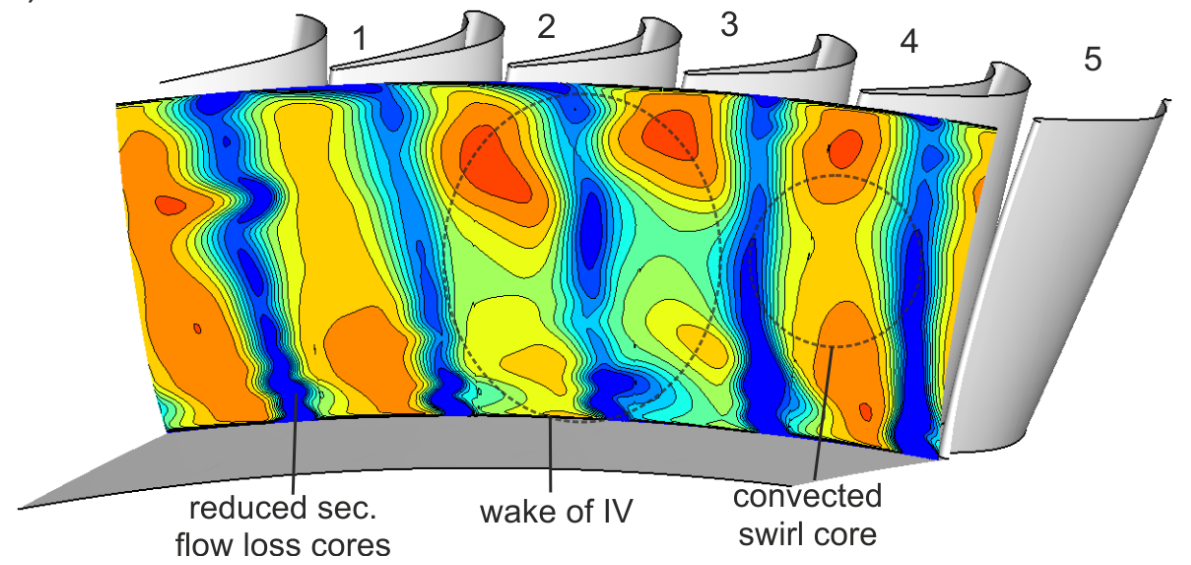

Figure 8-24: Instantaneous entropy function at 33\% span (top) and axial cut downstream of rotor (bottom) for profiled integrated vane stage simulation with swirl 
Most notably it can be seen that the rotor hub secondary flow loss cores (seen in Figure 8-17) are considerably reduced. This is caused by the more favourable rotor inlet flow conditions obtained through the vane profiling, with reduced underturning and overturning between $0 \%-25 \%$ and $40 \%-80 \%$ of span respectively. A look at the rotor hub instantaneous streamtraces for the integrated and profiled integrated vane, as shown in Figure 8-25, allows for a better qualitative understanding. It can be seen that the rotor inlet flow is more uniform for the profiled integrated vane, and the horse-shoe vortex system as a result more comparable between the rotor passages than in the case of the 'normal' integrated vane.

a) Integrated vane rotor - with swirl inlet

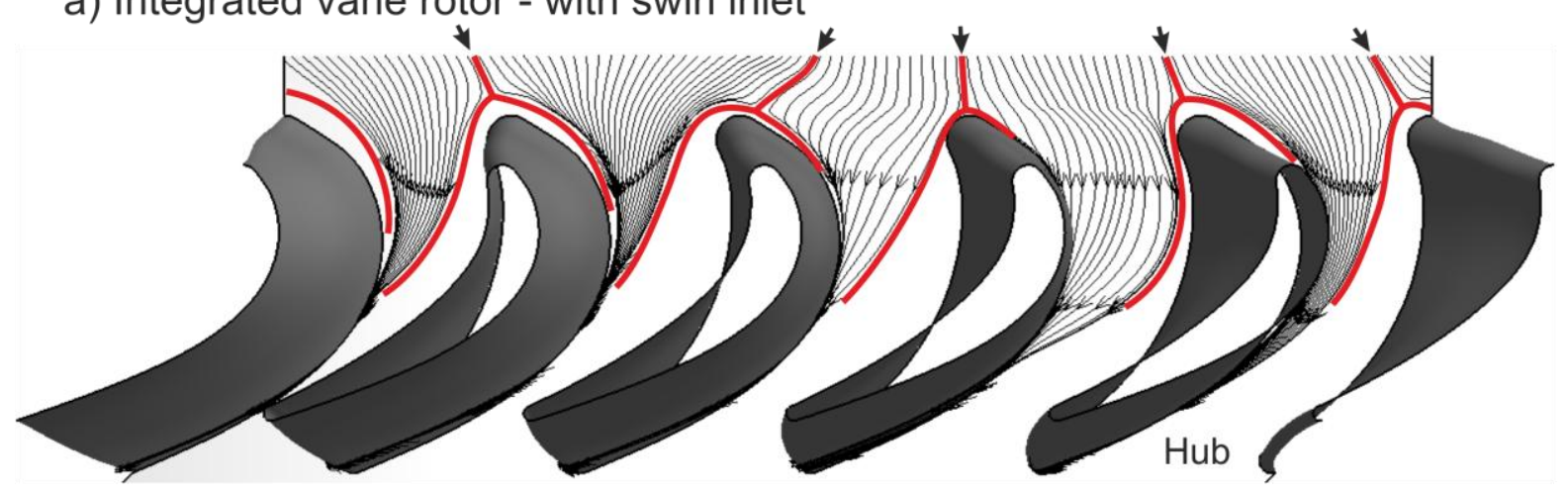

b) Profiled integrated vane rotor - with swirl inlet

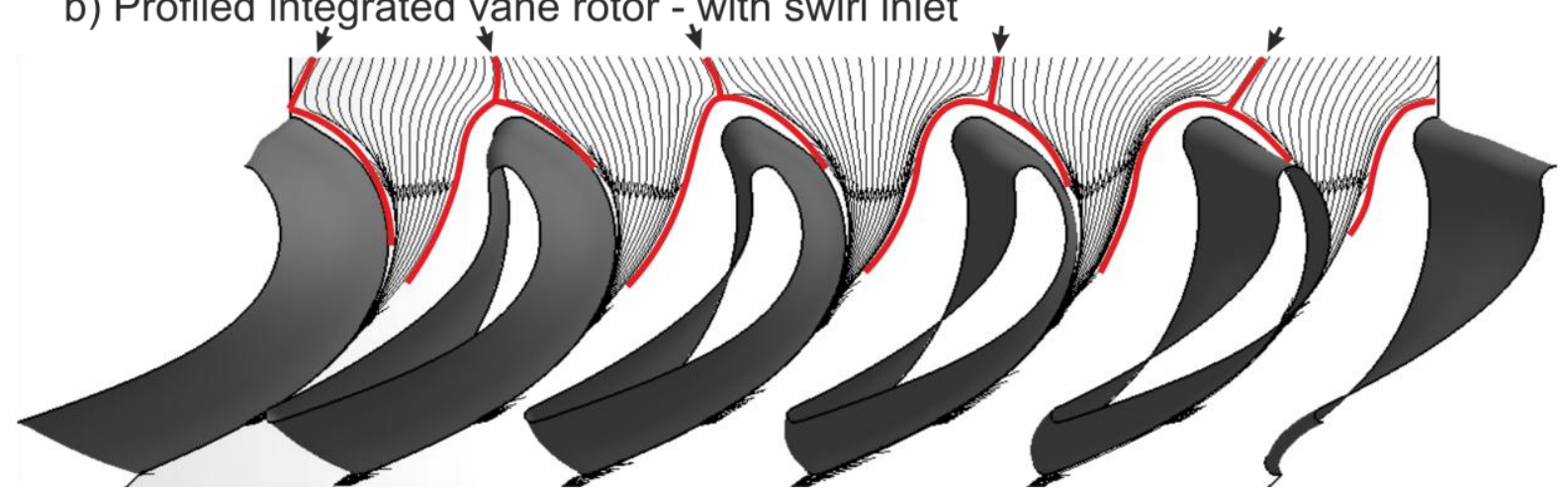

Figure 8-25: Instantaneous streamtraces on integrated (a) and profiled integrated (b) vane rotor for the swirl case

In order to compare the influence of profiling the integrated vane on the loss, Figure 8-26 shows the time- and pitchwise averaged entropy function profile at the rotor inlet and outlet. The graph shows that the vane profiling reduces aerodynamic loss downstream of the stator. It furthermore verifies the loss reduction between $0 \%-30 \%$ 
of span downstream of the rotor due to the decreased underturning of the flow downstream of the profiled integrated vane and the reduced hub secondary flow strength. This results in the illustrated increase in stage efficiency (Figure 8-23). It can thus be concluded that the substantial impact of swirl on the integrated vane stage performance can be effectively mitigated by employing three-dimensional vane profiling. Even in the presence of inlet swirl, the integrated vane design still outperforms the conventional vane design by more than $0.9 \%$ in stage efficiency.

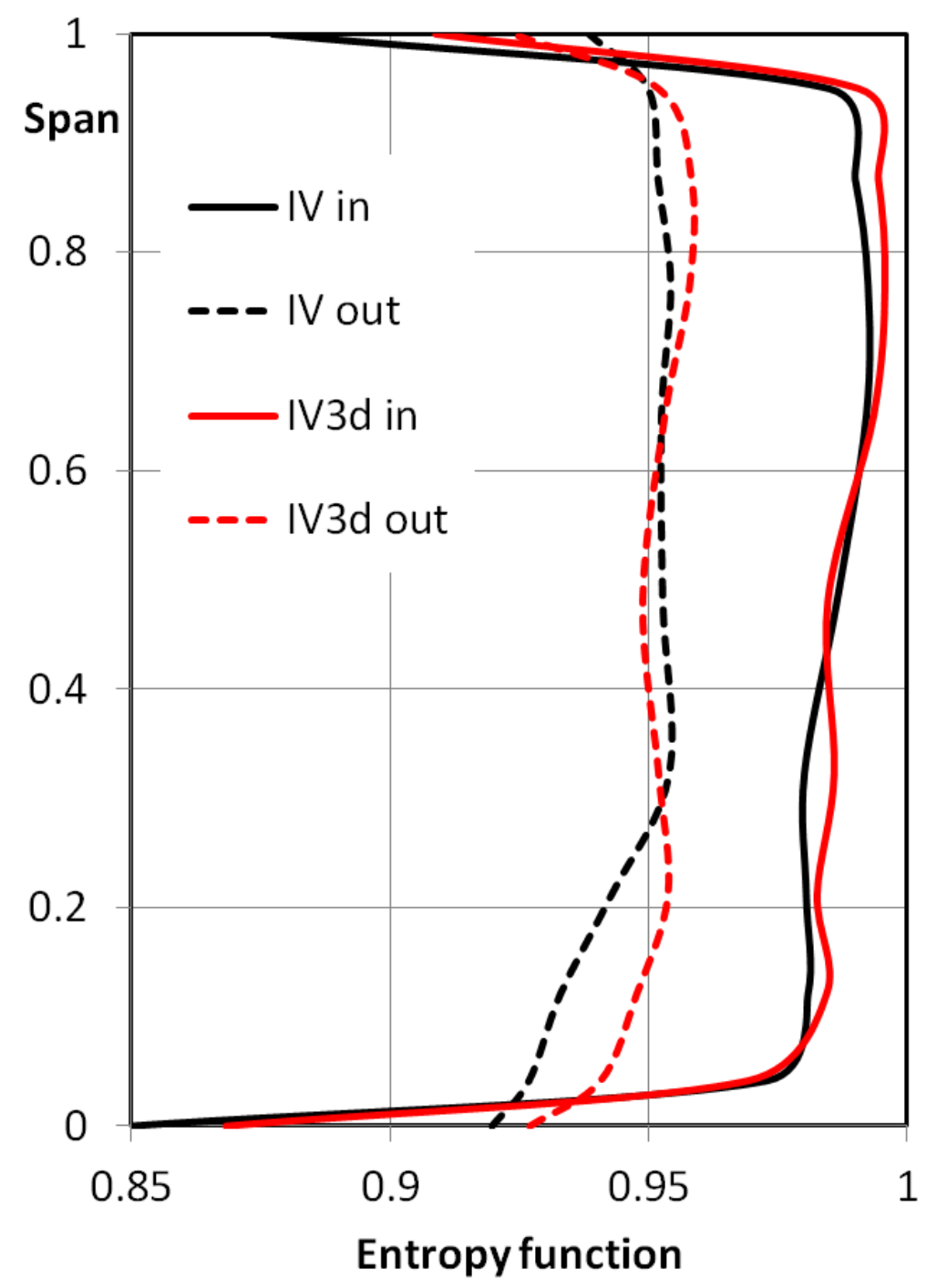

Figure 8-26: Time- and pitchwise-averaged entropy function at rotor inlet and outlet for the swirl case 


\section{Chapter 9: Cooling Performance of Integrated Vane}

\subsection{Abstract}

This chapter focuses on the cooling design, and experimental and numerical investigation of the integrated vane cooling characteristics. The integrated and conventional vane endwall cooling is achieved through continuous slots in the endwall, upstream of the vane. The integrated vane surfaces are cooled through two slots in the combustor wall, one for cooling the pressure and one for cooling the suction side. The cooling plena that are attached to the cooling system are developed using numerical simulations with the goal to achieve a uniform coolant supply. The numerical cooling effectiveness predictions are validated by experimental measurements. In summary, it is shown that the lack of a passagecentral vane (and thus removal of the horse-shoe vortex system) leads to a pitchwise more uniform static pressure distribution, and thus higher and more uniform endwall cooling effectiveness. Furthermore it can be seen that the leading edge cooling design is able to achieve high coolant coverage of the suction surface. Close attention needs to be paid to the sealing of the gap between combustor wall and integrated vane in order to achieve adequate cooling of the integrated vane pressure surface.

\subsection{Cooling design}

The cooling system of the combustor-vane interface was developed by conducting a numerical study using the TBLOCK solver. The cooling geometry for hub and casing endwalls is identical and shown in Figure 9-1, detail a). 


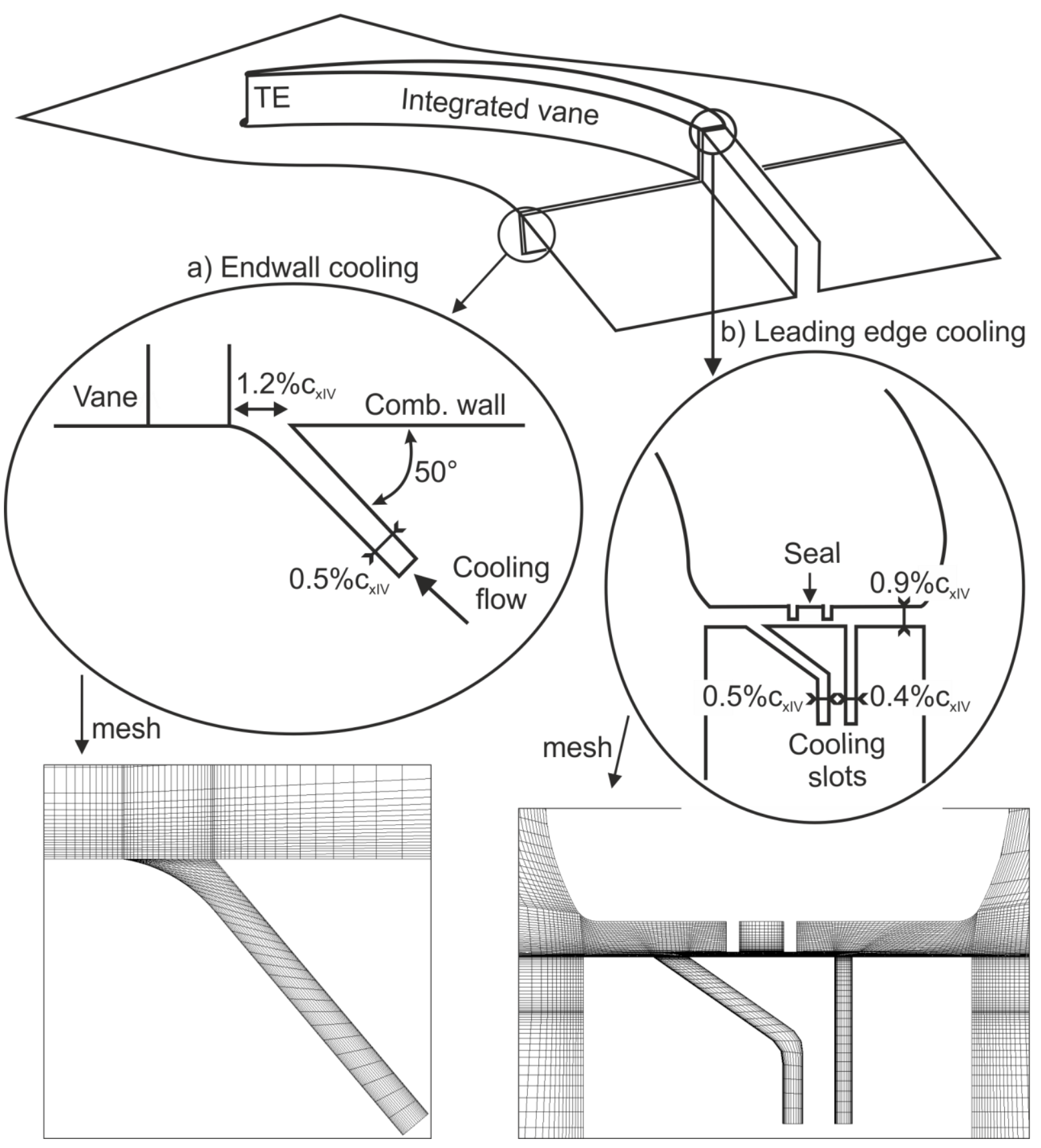

Figure 9-1: Schematic of integrated vane's endwall and leading edge cooling geometry and mesh

Endwall cooling is thus achieved with one slot inclined at $50^{\circ}$ with respect to axial flow. This resembles a simplification compared to a more realistic geometry with two cooling slots which will be briefly discussed at the end of the chapter. The simplification was needed for manufacturing (stereolithography's minimum manufacturing feature size) and mechanical integrity (thin walls will break due to 
pressure difference) reasons. The proximity of the integrated vane's leading edge to the transition duct wall trailing edge allows one to use continuous cooling slots in the combustor wall. The detailed geometry is shown in Figure 9-1, detail b). Figure 9-1 furthermore shows the mesh of the endwall and leading edge cooling. The mesh size for conventional and integrated vane design with cooling is 5.2 million and 6.3 million respectively.

Figure 9-2 shows pressure contours of the cooling slots exhausting from the transition duct wall trailing edge onto the integrated vane leading edge.

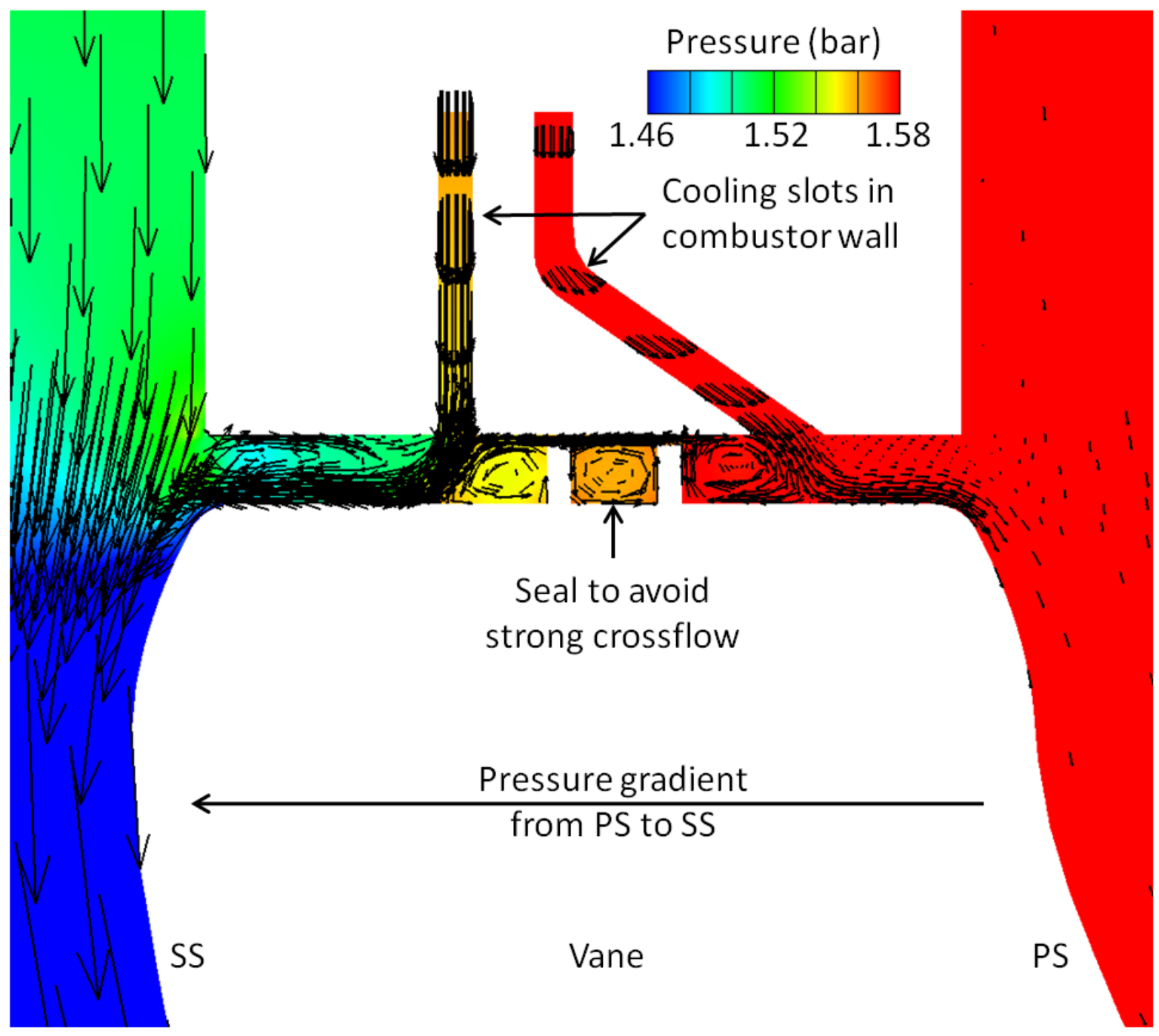

Figure 9-2: Predicted static pressure contour of integrated vane leading edge cooling design and seal at midspan; velocity vectors 
The cooling geometry consists of two slots, one for cooling the vane's pressure side and one for cooling the vane's suction side. The slot for the pressure side cooling has an angle of $45^{\circ}$ to counteract the pressure gradient from pressure to suction side in the gap between transition duct wall and integrated vane leading edge. Furthermore, a seal was designed in the gap to prevent a crossflow from pressure to suction side that could potentially affect the cooling of the vane's pressure surface. The flow pattern typical of a labyrinth seal is developed within the seal. It results in an intended pressure drop across the seal and thus helps to minimize the crossflow within the gap.

In order to provide the cooling for the two leading edge slots, a feeding plenum was developed. The flow solver Ansys Fluent was used for this in order to not constrain the geometry to simplistic designs by using unstructured meshing. The part's aim was to allow easy attachment to the cooling system, integrate smoothly into the combustor wall, and to consist of a cooling chamber large enough to provide sufficient and uniform coolant to the leading edge cooling slots. Figure 9-3 shows the evolution of the geometry of, and streamtraces in the feeding plenum for the leading edge cooling design, and the stagnation pressure in the final version. Option 1 resulted in a large vortex within the final plenum which could not be eliminated entirely by inclining two of the feeding holes in option 2. Options 1 and 2 did not result in a spanwise homogeneous pressure and velocity distribution. Option 4 eliminated the vortex of option 3 in the top right corner by rounding off its edge, and lead to a uniform exit pressure and velocity distribution. Option 4 was thus chosen as the final geometry. The inlet is fed through insulated plastic tubing by the cooling system. 11 holes are used to distribute the cooling flow homogeneously along the span. The coolant is finally ejected through two slots, one for cooling the integrated vane's pressure surface and one for cooling the integrated vane's suction surface. 


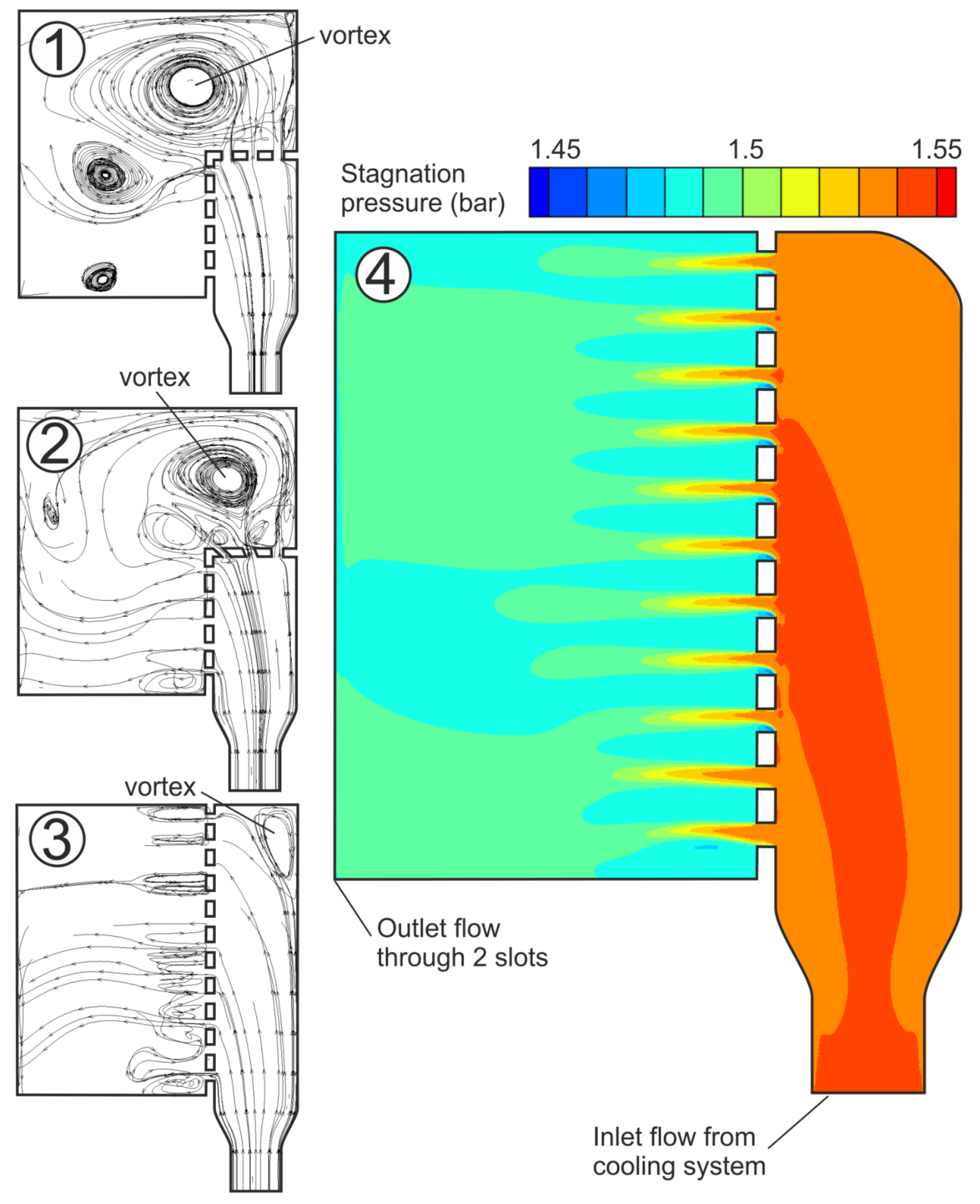

Figure 9-3: Predicted stagnation pressure in feeding plenum for leading edge cooling design

A further cooling box was devised for cooling the conventional and integrated vanes endwalls. As for the leading edge cooling plenum, Ansys Fluent was used due to the complex geometry. Figure 9-4 shows the CAD geometry of the cooling box. Coolant 
enters the chamber through three feeds which are connected to insulated tubes of the cooling system. Each of these feeds consists of 10 jet-holes ( 3 at the top of each feed) in order to distribute the flow evenly in the chamber. The jets of one feed can clearly be seen in the stagnation pressure plot in Figure 9-4. The design achieves a uniform pressure and velocity distribution at the exit of the outlet slot.

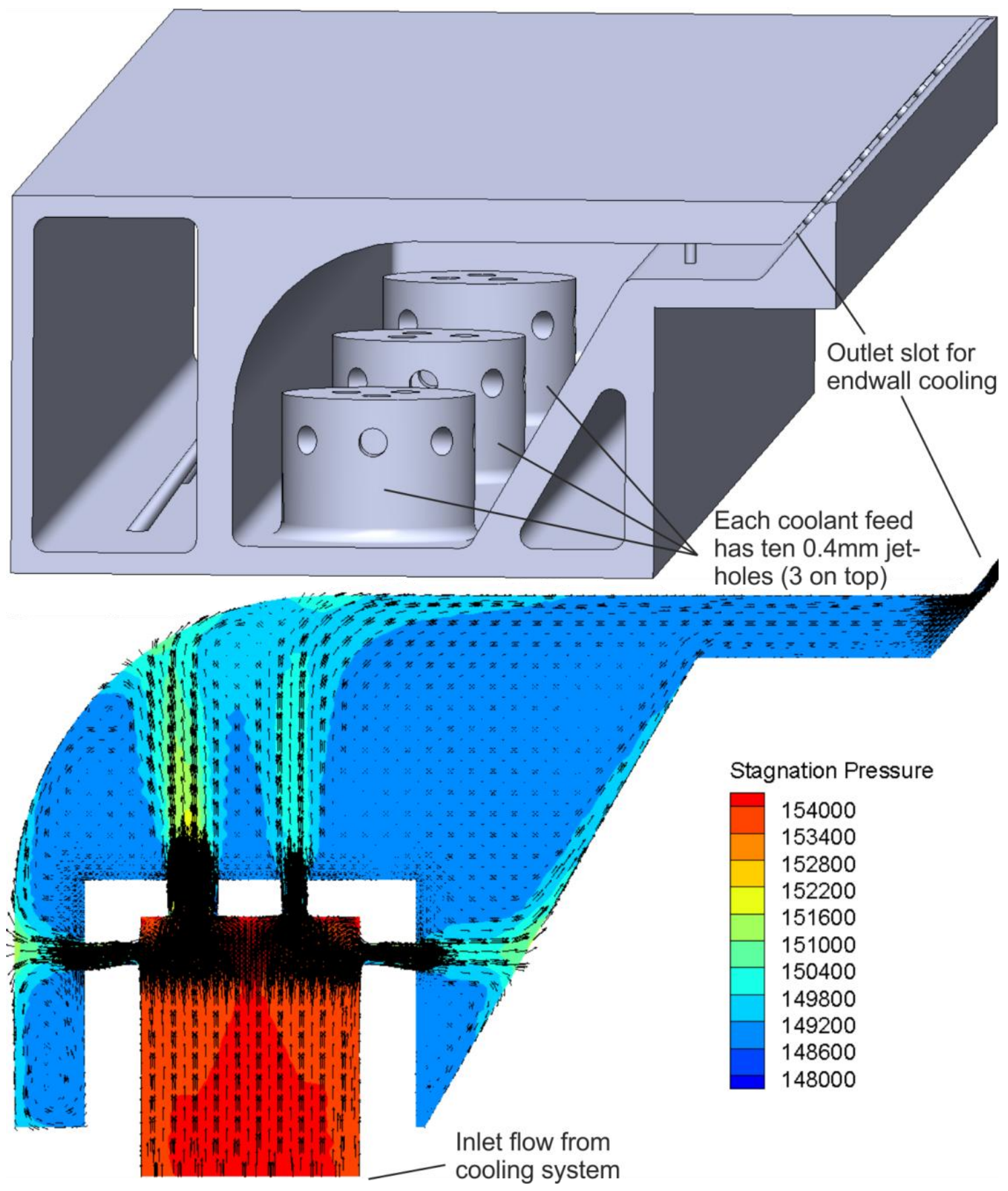

Figure 9-4: Geometry of cooling box used for endwall cooling (top), and slice of predicted stagnation pressure with velocity vectors (bottom) 
The cooling boxes were manufactured using stereolithography and equipped with thermocouples and pressure tappings in order to measure the conditions inside the coolant plenums. A photo of the cooling box for leading edge cooling and the cooling box for endwall cooling can be seen in Figure 9-5. The coolant feeds can easily be connected to the cooling system when needed for experimental measurements.

a) Cooling box for leading edge cooling

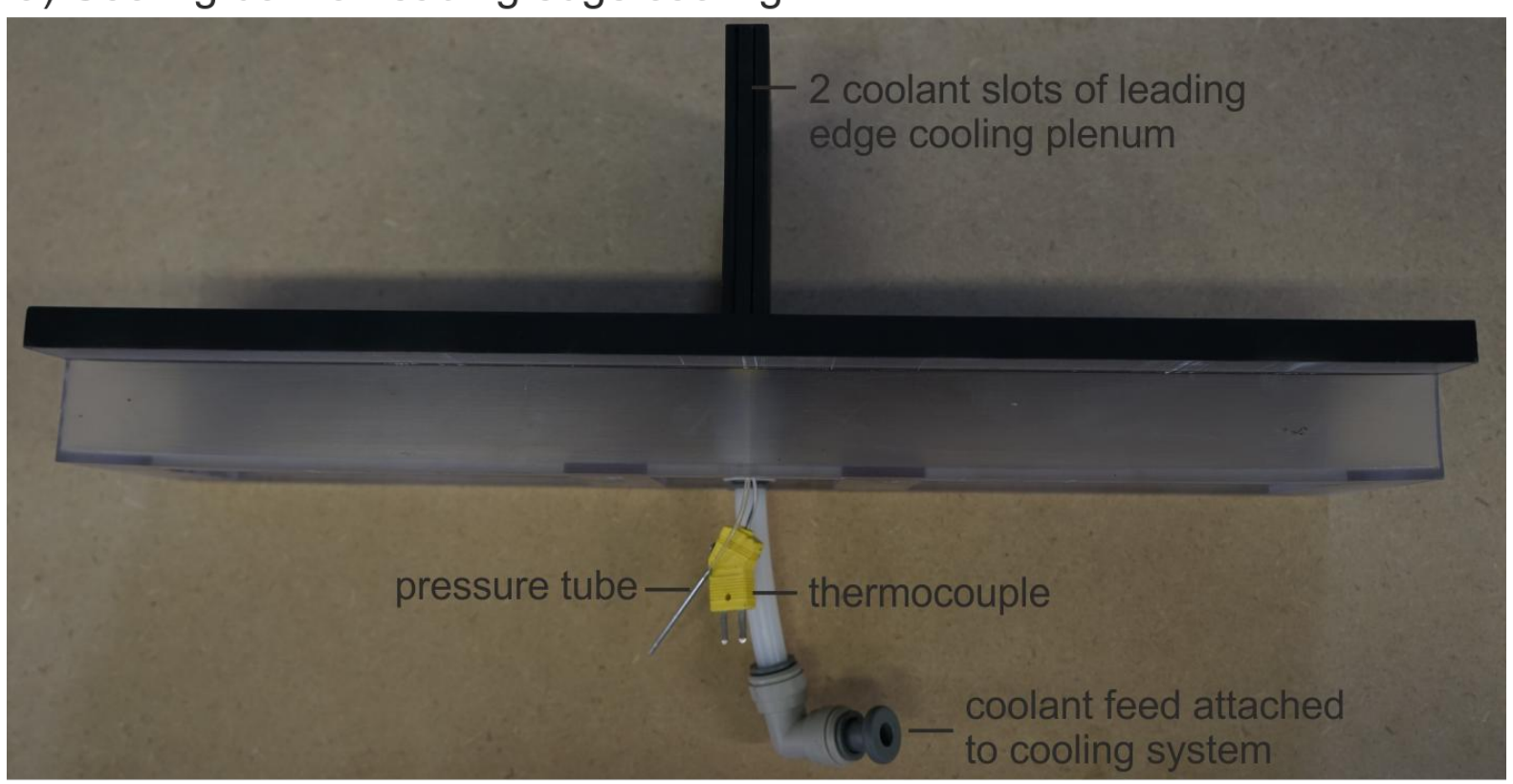

b) Cooling box for endwall cooling

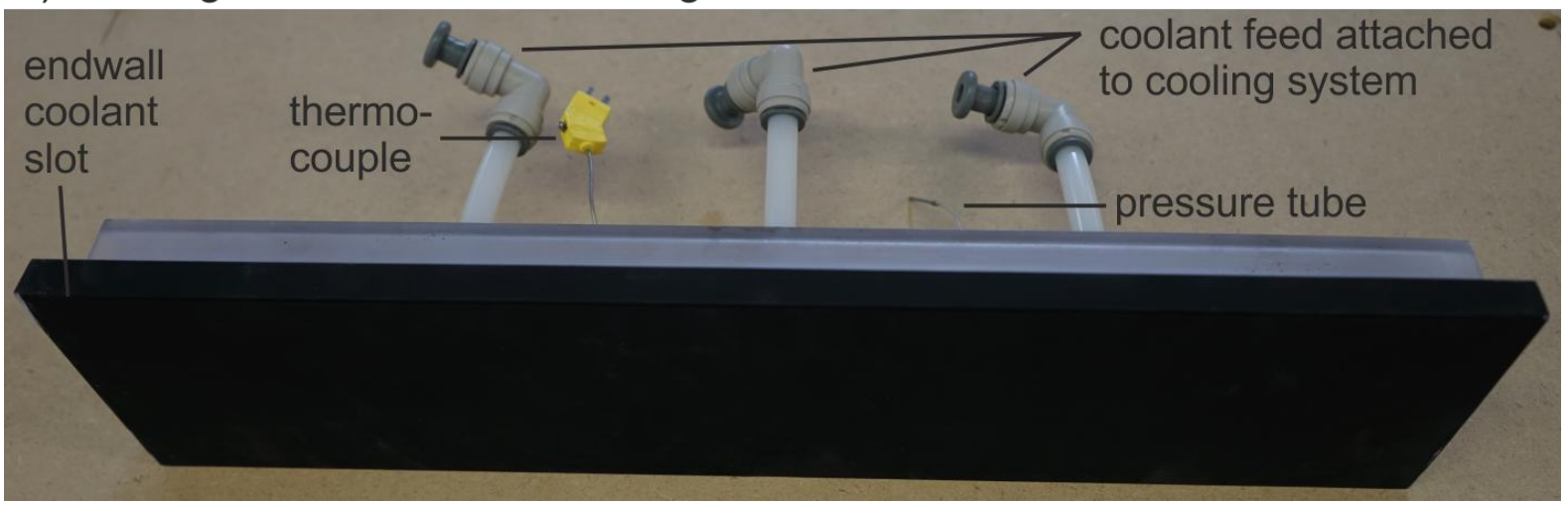

Figure 9-5: Photo of cooling box for leading edge cooling (a) and endwall cooling (b)

\subsection{Experimental procedure and conditions}

The experimental cooling campaign is undertaken in order to compare cooling effectiveness between conventional and integrated vanes. The coolant flow from the 
cooling system is either attached to the leading edge cooling box (for integrated vane leading edge cooling) or to the hub endwall cooling box (for conventional and integrated vane endwall cooling). The experiments are performed for several different views in order to resolve the suction and pressure surfaces, leading edges and endwalls. Prior to each run on the experimental facility, the cooling system needs to be started for approximately 20-30 minutes (detaching the coolant tubing from the cooling boxes) to cool down the whole cooling system to a steady temperature. This is necessary to minimize the heat pickup within the system and tubing as much as possible.

Figure 9-14 shows the temperatures (measured by thermocouples) of the mainstream flow, inside of the cooling box and inside of the cooling plenum (which is attached to the vortex tubes) during an experimental cooling run.

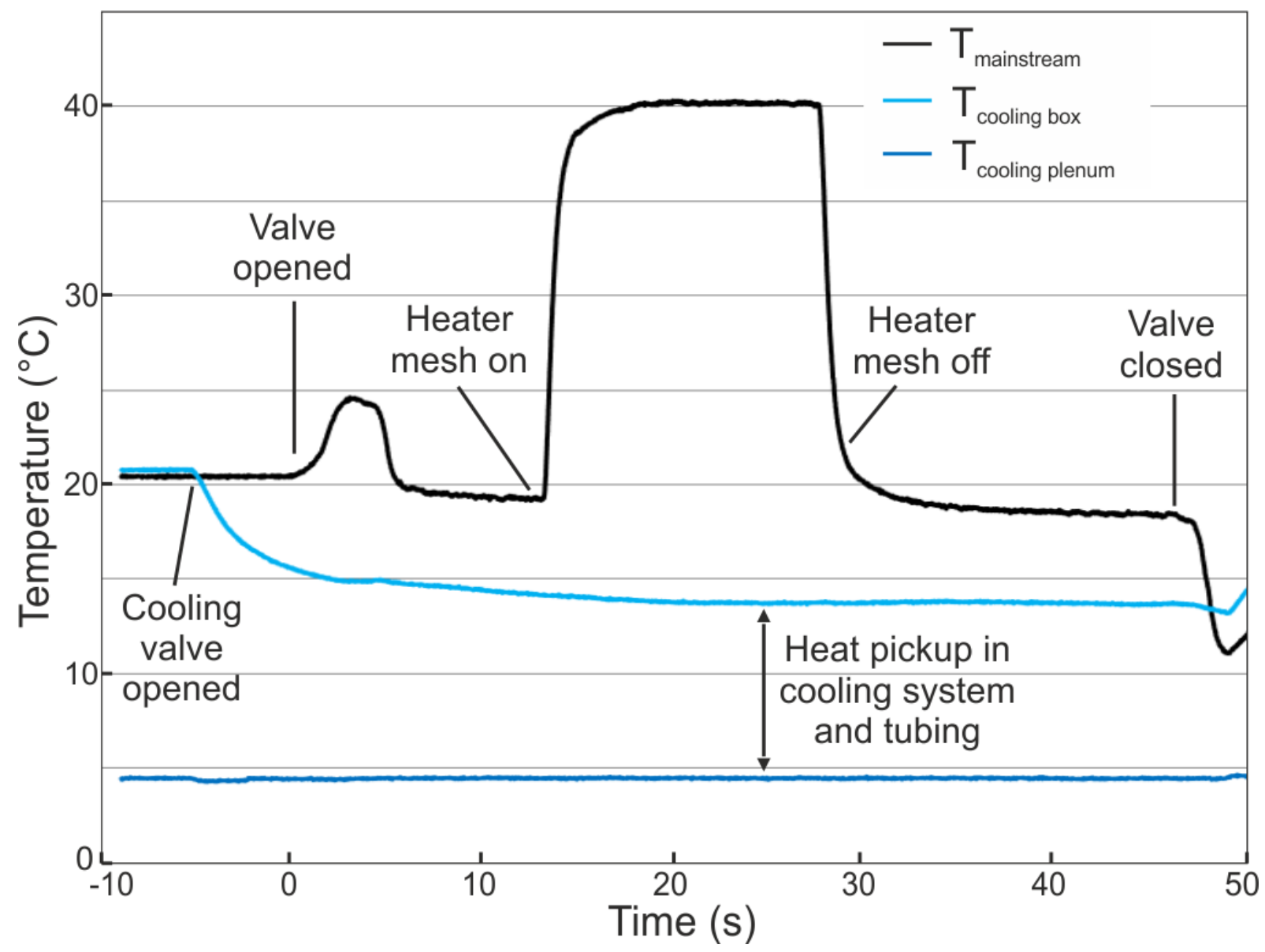

Figure 9-6: Measured temperatures during a cooling run on the experimental facility 
It can be seen that the ball valve at the exit of the cooling system (see Figure 3-13) is opened before the valve of the mainstream opens. This is done to fill the cooling box with coolant, thus pressurizing it and reducing the pressure difference across the cooling boxes endwall (thus reducing the risk of breakage). At the time the heater mesh is turned on, the temperature in the cooling box has reached nearly steady state. A temperature difference of more than $25 \mathrm{~K}$ is thus obtained between the mainstream and coolant flow. The graph however also highlights that the temperature difference between the cooling plenum of the cooling system and the cooling box is significant. Despite extensive use of insulation of the cooling system and tubing, the coolant picks up heat on its way to the cooling boxes and is thus approximately $10 \mathrm{~K}$ warmer than the air exiting the vortex tubes.

Figure 9-7 similarly shows a graph of the pressures obtained upstream of the turbulence grid, inside of the combustor transition duct and inside of the leading edge cooling box during an experimental test run with cooling. It can be seen how the pressure inside of the cooling box rises (prior to the opening of the mainstream valve) as soon as the valve of the cooling system is partially opened. It is fully opened during the rise of mainstream pressure. It can be seen how the total pressure drops from 1.65 bar to 1.5 bar across the turbulence grid. The graph furthermore shows that the pressure inside of the cooling box follows the overall trends of the mainstream pressure and its level is between the total and static pressure inside of the transition duct during the time the heater mesh is turned on. This pressure is the maximum attainable coolant pressure for the current cooling system, and only a redesign would allow for higher coolant pressures and thus massflow rates. 


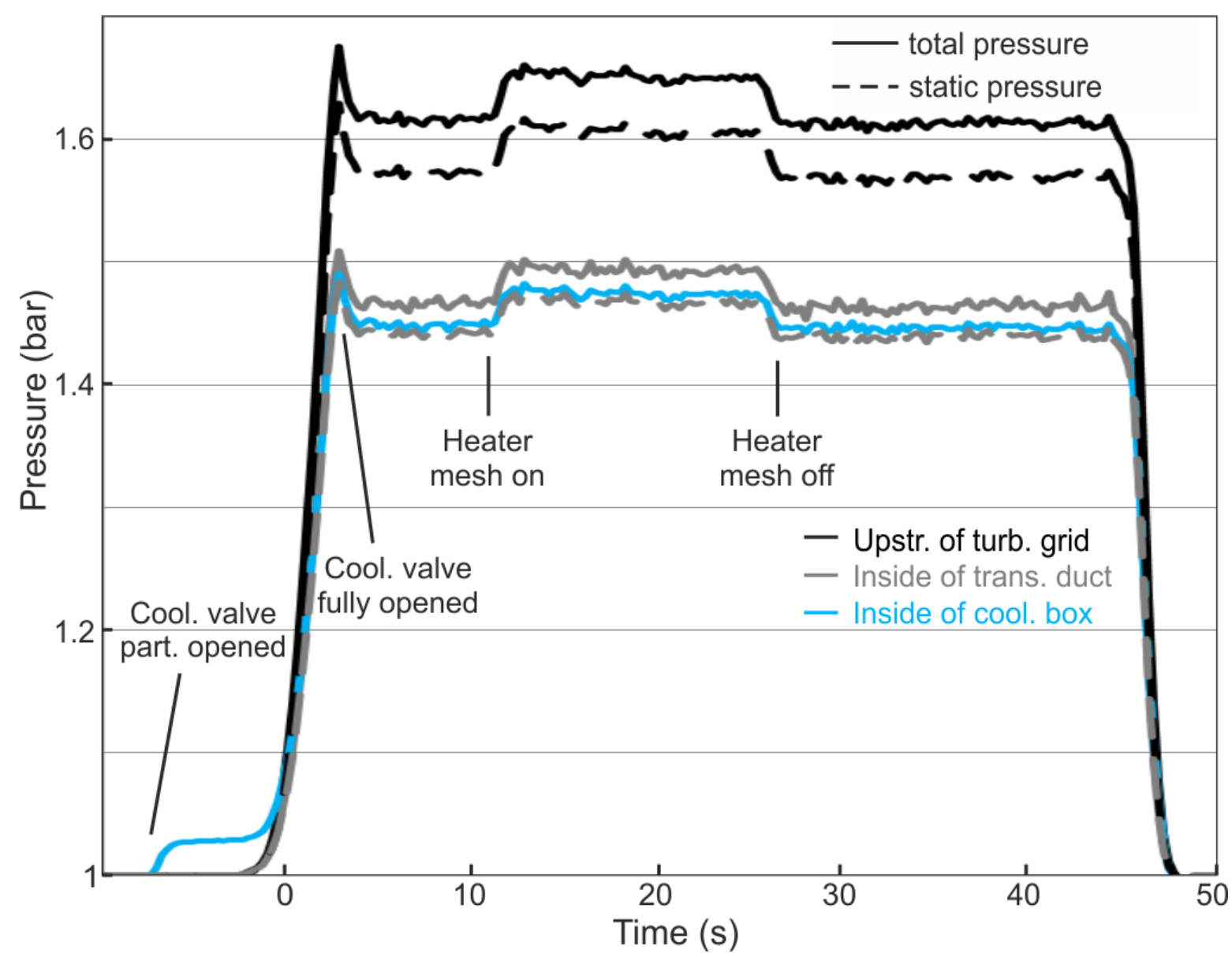

Figure 9-7: Measured pressures in the working section during an experimental cooling run

\subsection{Experimental and numerical cooling of conventional vane}

Conventional vanes use showerhead film cooling for cooling the vane's leading edge (in contrast to the leading edge cooling system for the integrated vane) and further cooling hole rows on the pressure and suction surfaces. This study did however not perform any cooling hole experiments, instead only investigating endwall cooling performance. Figure 9-8 thus shows the predicted cooling effectiveness of the conventional vane design with endwall cooling, for an upstream (top) and a downstream (bottom) view. It can be seen how the coolant exits the endwall cooling slot close to the shielded and unshielded vanes leading edges on the suctions sides. This leaves large parts of the hub endwall uncooled. The trace of cooling 
effectiveness quickly narrows, and the flow is convected towards the suction side. The coolant is collected by the secondary flows and migrates up the suction surfaces. A slight trace of coolant can thus be seen with a similar appearance to suction surface HTC separation lines.

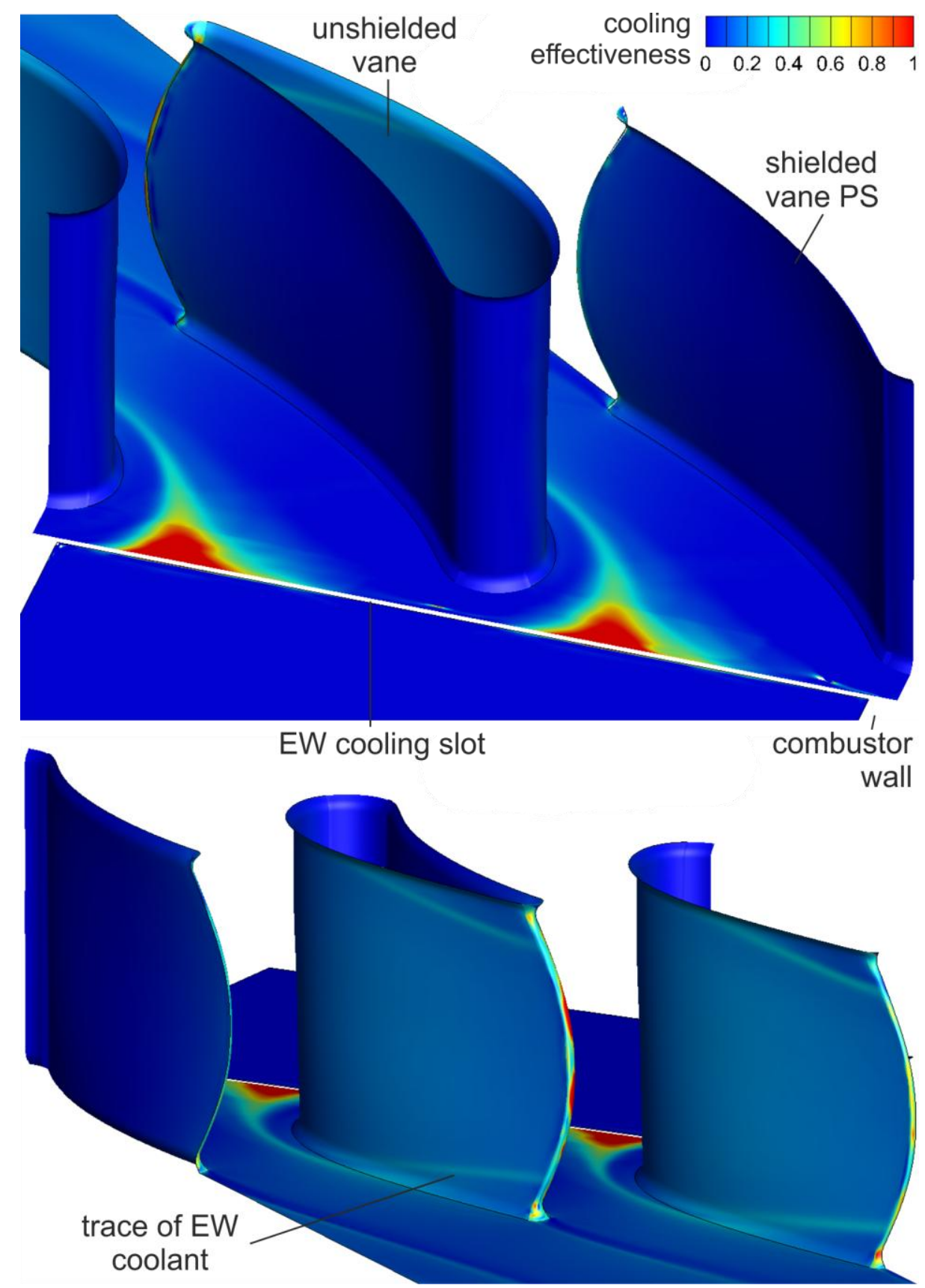

Figure 9-8: Predicted cooling effectiveness of conventional vane design with endwall cooling

In order to validate the cooling effectiveness distributions predicted by the numerical simulations, experimental measurements were conducted for several views using the 
infrared camera. Figure 9-9 shows the cooling effectiveness on the conventional vane's hub endwall - focussing on the region close to the shielded vane's leading edge as seen in the schematic's grey ellipse - with endwall cooling.

\section{Cooling effectiveness}
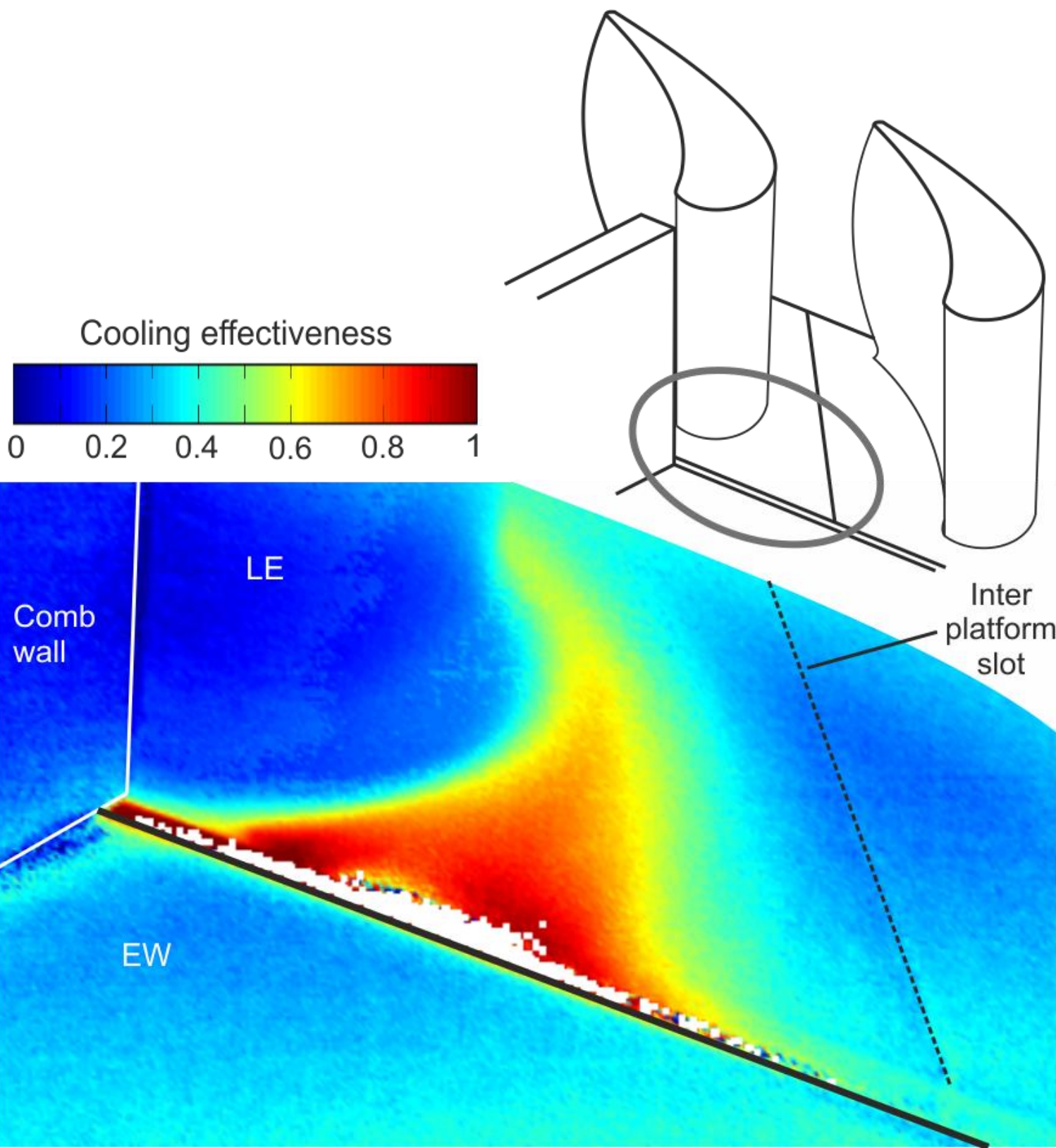

Figure 9-9: Measured cooling effectiveness on conventional vane hub endwall with endwall cooling for a suction side view

It can be seen that the coolant is ejected in the vicinity of the shielded conventional vane's leading edge towards the suction side since this is the region of the lowest static pressure. The coolant trace narrows due to the passage's pressure gradient 
and is convected towards the suction surface. The distribution agrees very well with the predicted cooling effectiveness. Figure 9-10 similarly shows the cooling effectiveness on the conventional vane's hub endwall focussing on the region close to the unshielded vane's leading edge as indicated in the schematic's grey ellipse.
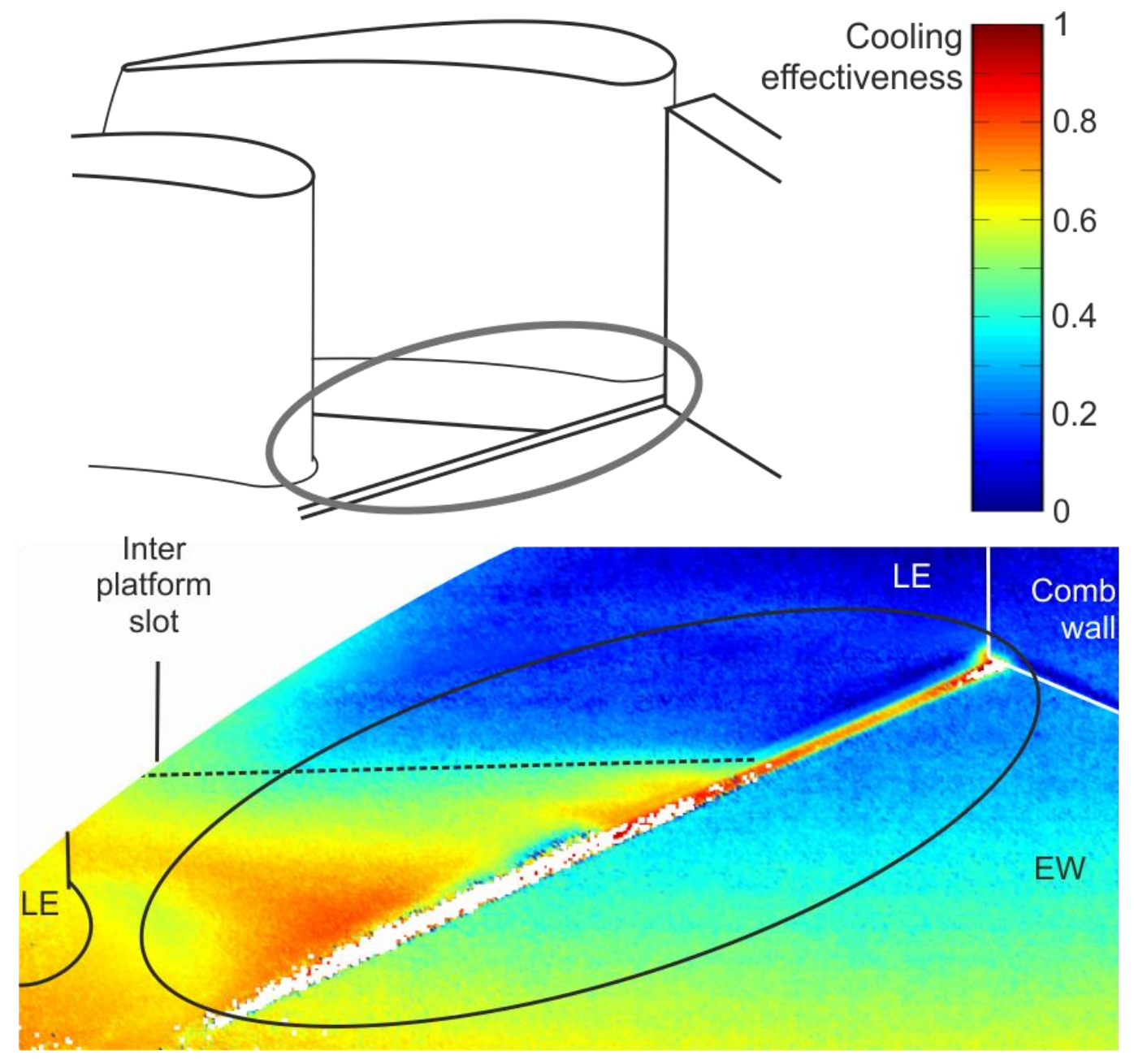

Figure 9-10: Measured cooling effectiveness on conventional vane hub endwall with endwall cooling for a pressure side view

The coolant similarly exits the endwall slot in the vicinity of the unshielded vane's leading edge towards the suction side and the distribution of cooling effectiveness is comparable in its form and levels to the trace seen in the measurements of Figure 9-9 and the predictions of Figure 9-8. The endwall cooling design thus covers only a limited part of the passage's pitch, as predicted by the CFD-simulation. It is noted that only the region marked by the black lined ellipse in Figure 9-10 should be 
evaluated since the values outside of this ellipse are affected by reflections of the infrared window. Figure 9-9 and Figure 9-10 showed upstream coolant distributions, while Figure 9-11 and Figure 9-12 will show downstream distributions, thus following the path of the coolant flow.

Figure 9-11 shows the measured cooling effectiveness on the conventional vane hub endwall - looking from downstream - with endwall cooling.

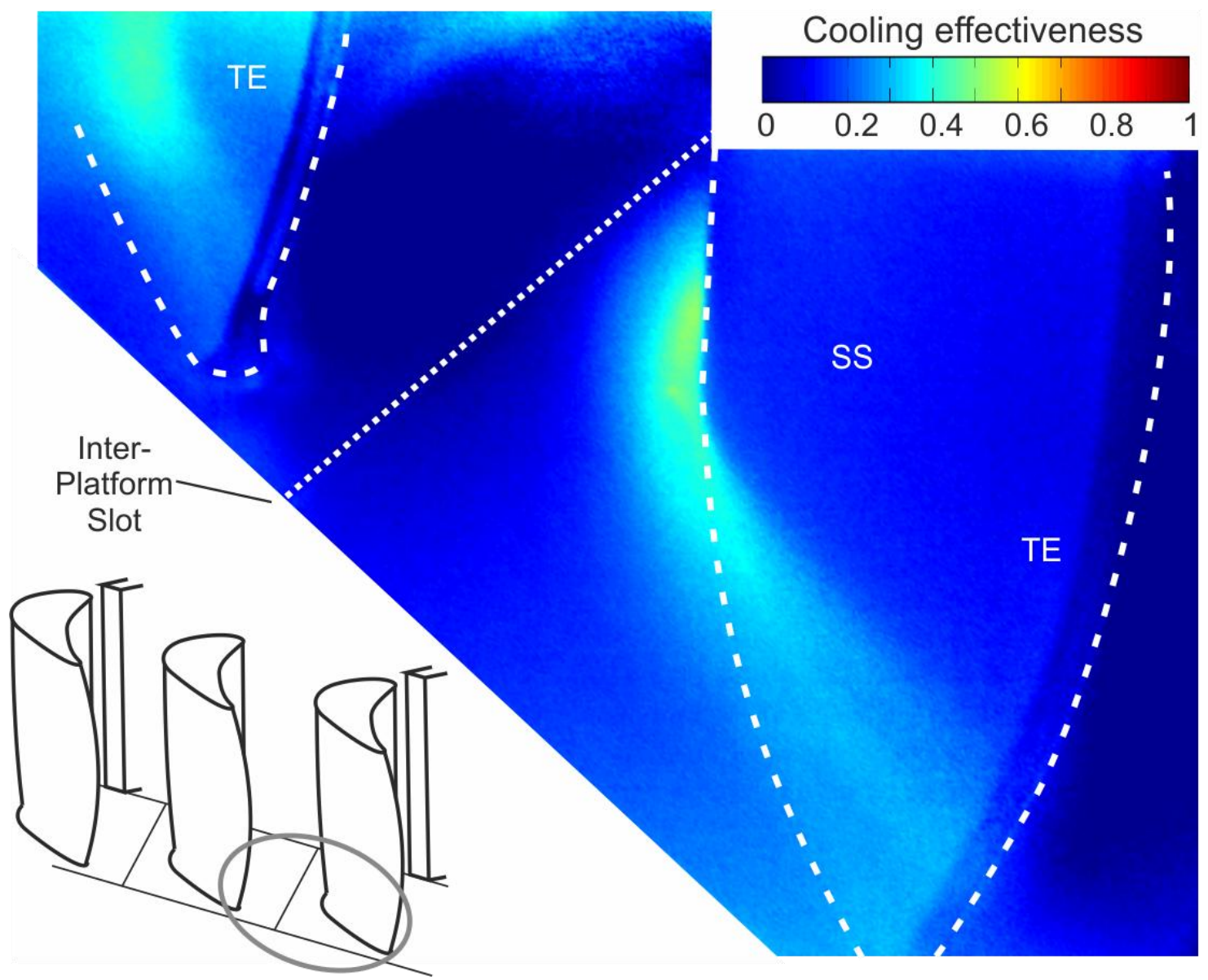

Figure 9-11: Measured cooling effectiveness on conventional vane hub endwall (looking from downstream) with endwall cooling

It can be seen how the coolant ejected from the upstream hub endwall slot is quickly convected towards the shielded vane's suction side and migrates up the suction surface. This leaves nearly the entire remainder of the hub endwall completely uncooled. The cooling effectiveness distribution on the suction surface can be seen clearly in Figure 9-12. It is likely that the coolant flow is picked up by the passage 
vortex as it subsequently follows the trajectory of the secondary flows onto the suction surface. The levels of cooling effectiveness are only slightly higher for the shielded conventional vane while the distributions of the shielded and unshielded conventional vanes suction surface cooling effectiveness distributions are qualitatively very similar. The slightly higher cooling effectiveness levels of the shielded vane are due to the decreased pressure behind the combustor wall, which leads to slightly increased coolant ejection. The levels of cooling effectiveness decrease towards the trailing edge due to the diffusion on the suction surface. Agreement with the numerical simulation is good, with only slightly higher cooling effectiveness levels for the measurements.
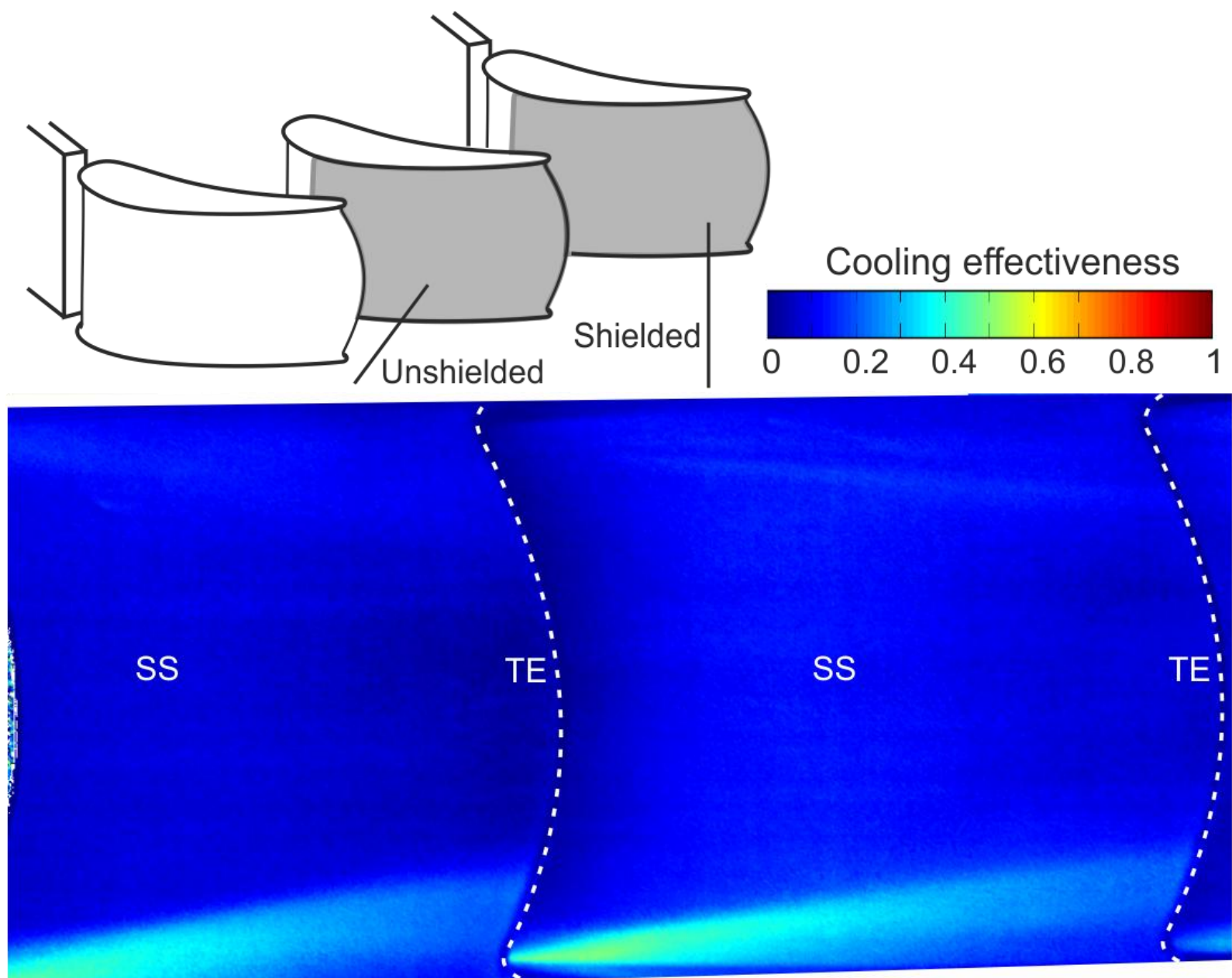

Figure 9-12: Measured cooling effectiveness on conventional vane suction surfaces with endwall cooling 
To summarize, it has been shown how the conventional vane design leads to a nonuniform pitchwise endwall coolant ejection. The coolant only covers a small fraction of the endwall due to the strong passage pressure gradient and horse-shoe vortex formation. Good agreement of the cooling effectiveness distribution between experimental measurements and numerical simulations was shown. The following subchapter will similarly investigate the experimental and numerical cooling effectiveness of the integrated vane in order to finally compare the conventional and integrated vane cooling performances.

\subsection{Experimental and numerical cooling of integrated vane}

Figure 9-13 shows the predicted cooling effectiveness of the integrated vane design with leading edge (top) and with endwall (bottom) cooling. This distinction is made not only to clearly separate their effects on the suction surface, but also due to a limited supply of coolant massflow rate in the experimental cooling system design. Figure 9-13a shows that the coolant ejected through the leading edge cooling slots is convected along the suction surface. This leads to the coverage in cooling effectiveness of nearly the entire suction surface, with levels decreasing from 1 at the leading edge to 0.3 at the trailing edge. A slight contraction towards the trailing edge is visible due to the migration of low-momentum endwall fluid up onto the suction surface. On the pressure side only the leading edge is cooled due to the strong pressure gradient from pressure to suction side (forcing the majority of coolant towards the suction side). Figure 9-13b shows how the coolant is ejected through the endwall cooling slot. The cooling effectiveness increases in pitchwise direction towards the suction surface due to the lowest static pressure in this region. The coolant is convected towards the suction side and migrates onto the suction surface 
following the path of the secondary flow. The endwall cooling thus covers the area of the suction surface that was left uncooled when only employing leading edge cooling.

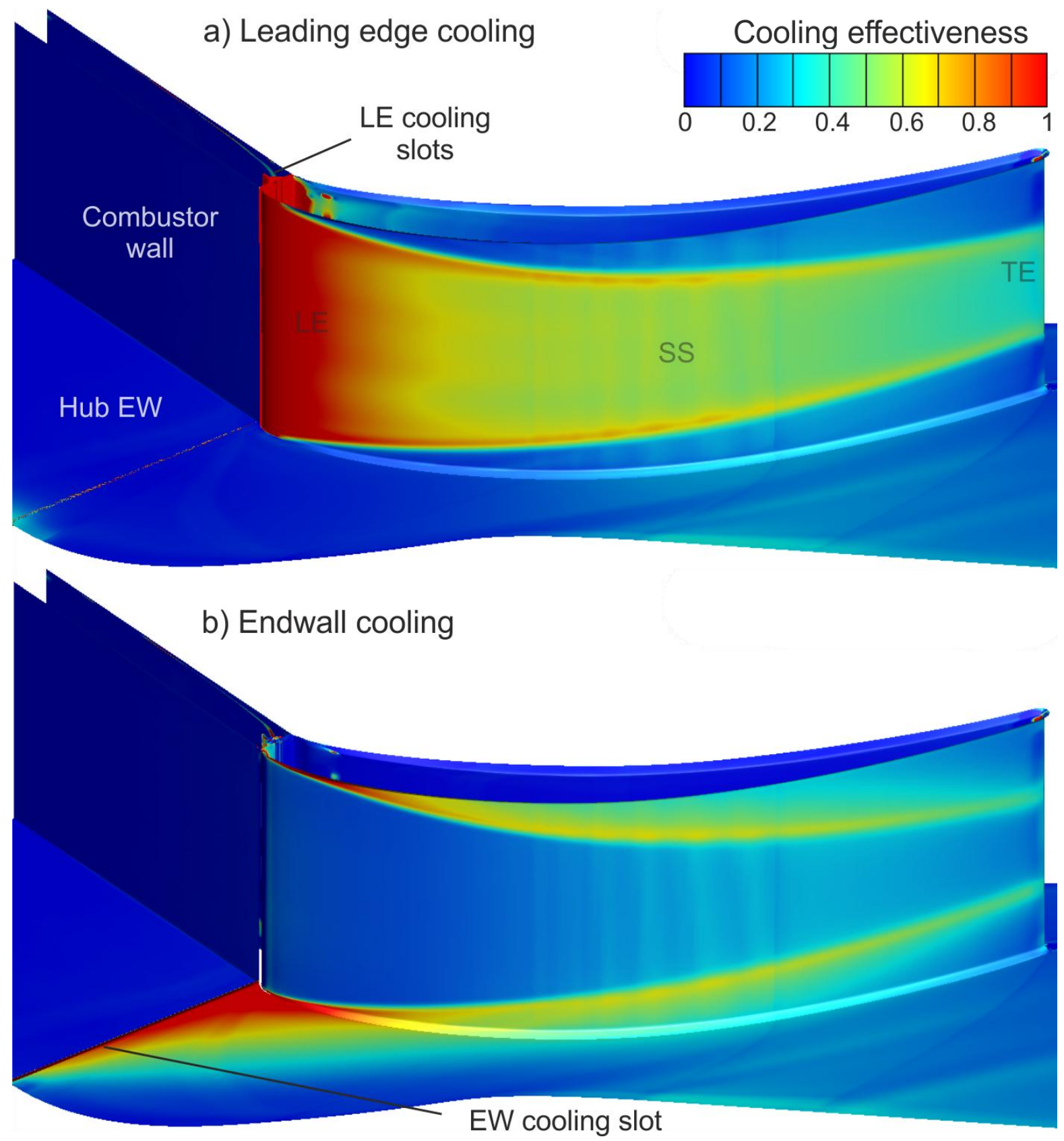

Figure 9-13: Predicted cooling effectiveness of integrated vane design with leading edge (a) and endwall (b) cooling

The following investigations will feature an experimental validation - showing various views with the infrared camera - of the numerical simulations. Figure 9-14 shows the measured cooling effectiveness on the integrated vane's leading edge with leading edge cooling. The cooling effectiveness on the leading edge achieves values around 
0.8 and is evenly distributed along the span, with only slightly higher levels towards the hub and casing. This gives confidence regarding a successful design (i.e. uniform plenum exit flow) of the leading edge coolant feed of Figure 9-3 (option 4). The levels of cooling effectiveness subsequently drop to $0.5-0.65$ towards the suction surface.

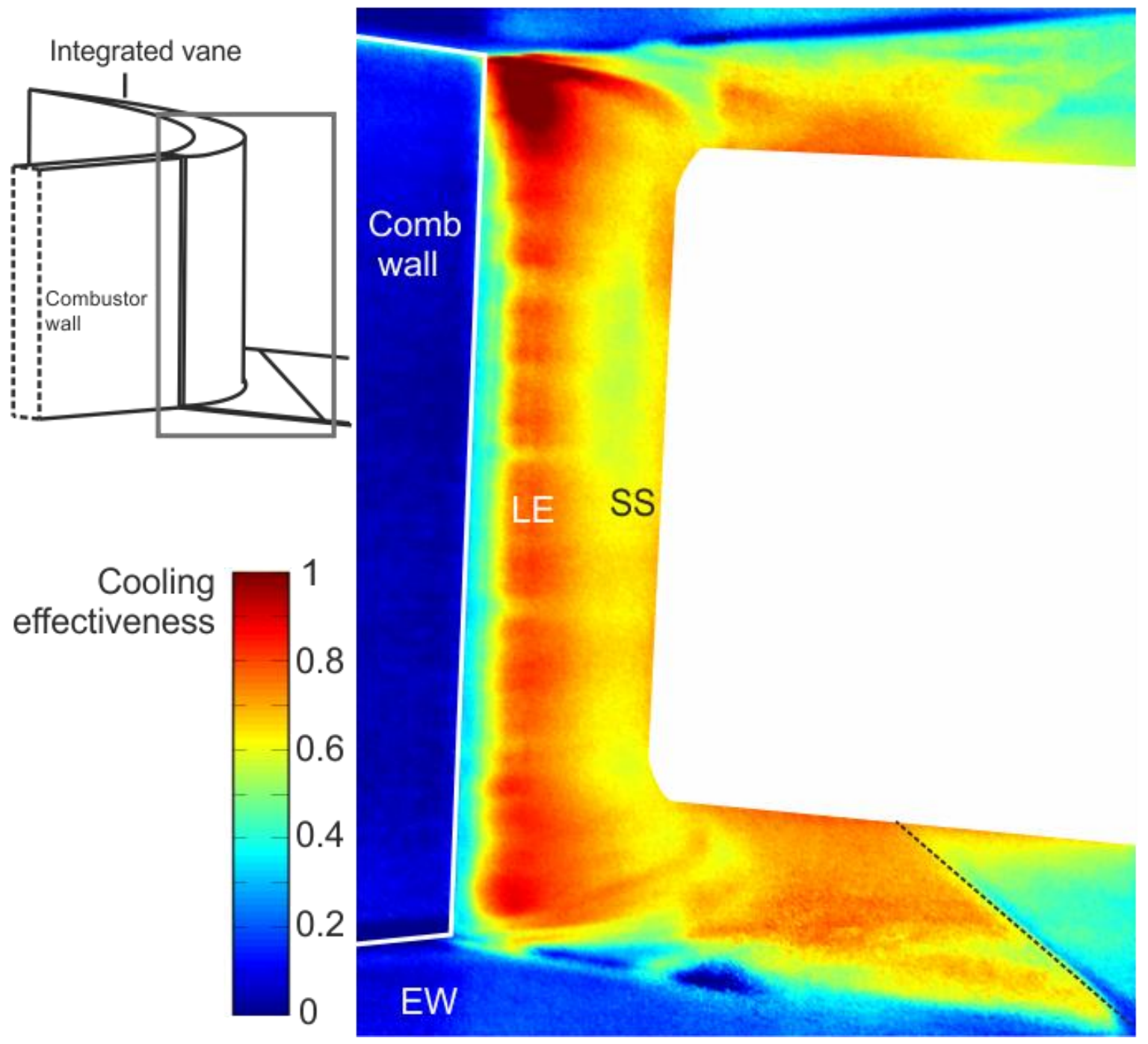

Figure 9-14: Measured cooling effectiveness on integrated vane leading edge with leading edge cooling

Figure 9-15 shows the measured cooling effectiveness on the integrated vane's suction surface with leading edge cooling. It can be seen that the coolant stays attached to the suction surface nearly until the trailing edge. Values of cooling effectiveness decrease from 0.55 in the region of peak velocities to 0.15 at the 
trailing edge. The region towards hub and casing in the area of the secondary flows remains uncooled.
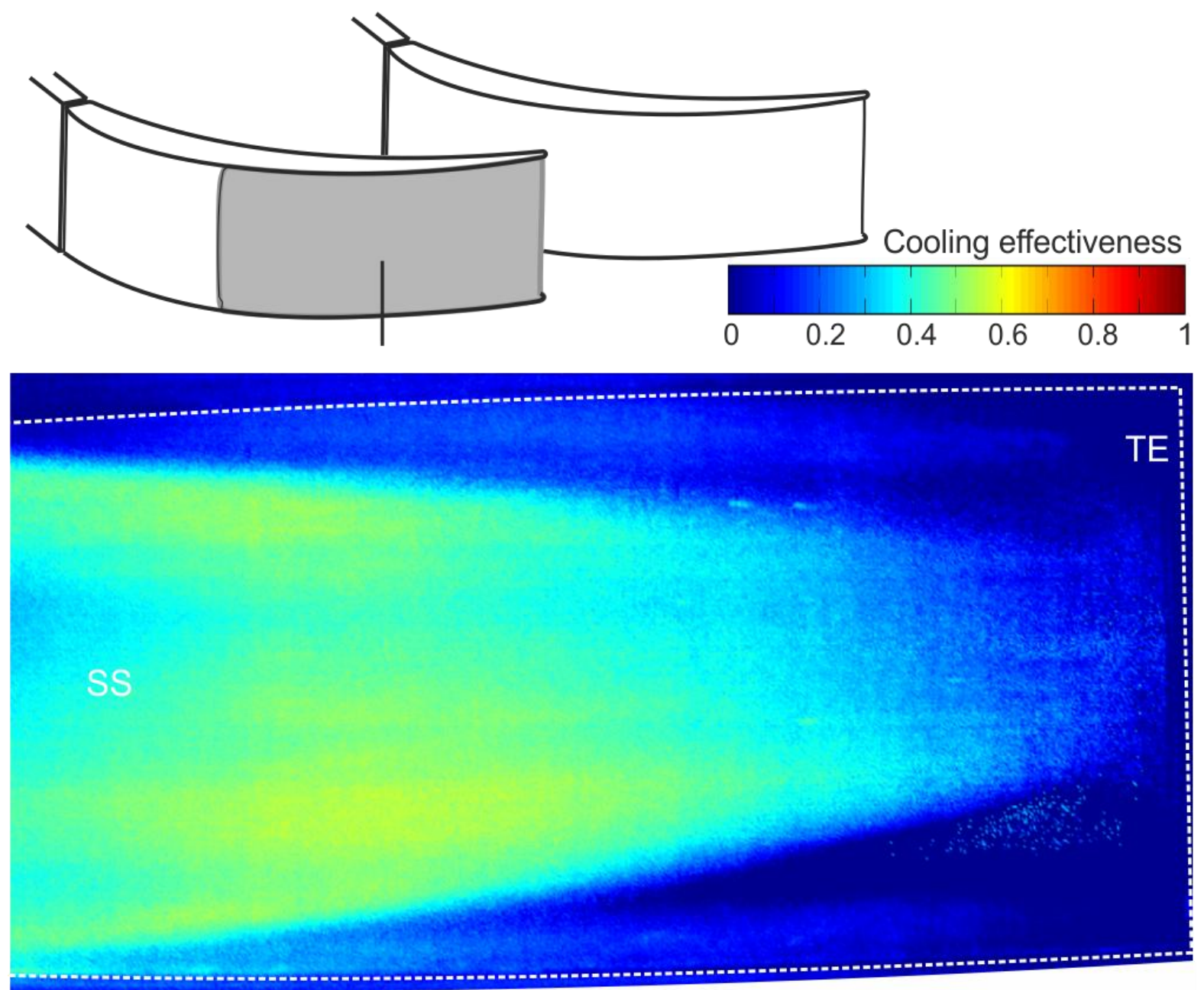

Figure 9-15: Measured cooling effectiveness on integrated vane suction surface with leading edge cooling

The experimental measurements with leading edge cooling thus agree qualitatively and quantitatively fairly well to the predictions of the CFD-simulation in Figure 9-13a. The following measurements will focus on validating the endwall cooling predictions.

Figure 9-16 shows the cooling effectiveness on the integrated vane's hub endwall obtained from experimental measurements with endwall cooling. It can be seen that coolant flow exits the endwall slot across the pitch. More coolant is ejected towards the suction side than towards the pressure side, since static pressure is lower at the 
suction side. The coolant stays attached to the hub endwall and is turned by the remaining flow. The measured cooling effectiveness levels are higher compared to the predictions of the CFD-simulations, indicating that the coolant flow stays attached to the endwall for longer. An irregularity in the cooling effectiveness can be seen in detail A, which can also be noticed in Figure $6-4 \mathrm{~b}$. It is suspected that this is due to a minor manufacturing deviation on the integrated vane's hub endwall that causes the coolant flow to bypass this location. This feature does not occur in the CFDsimulations.

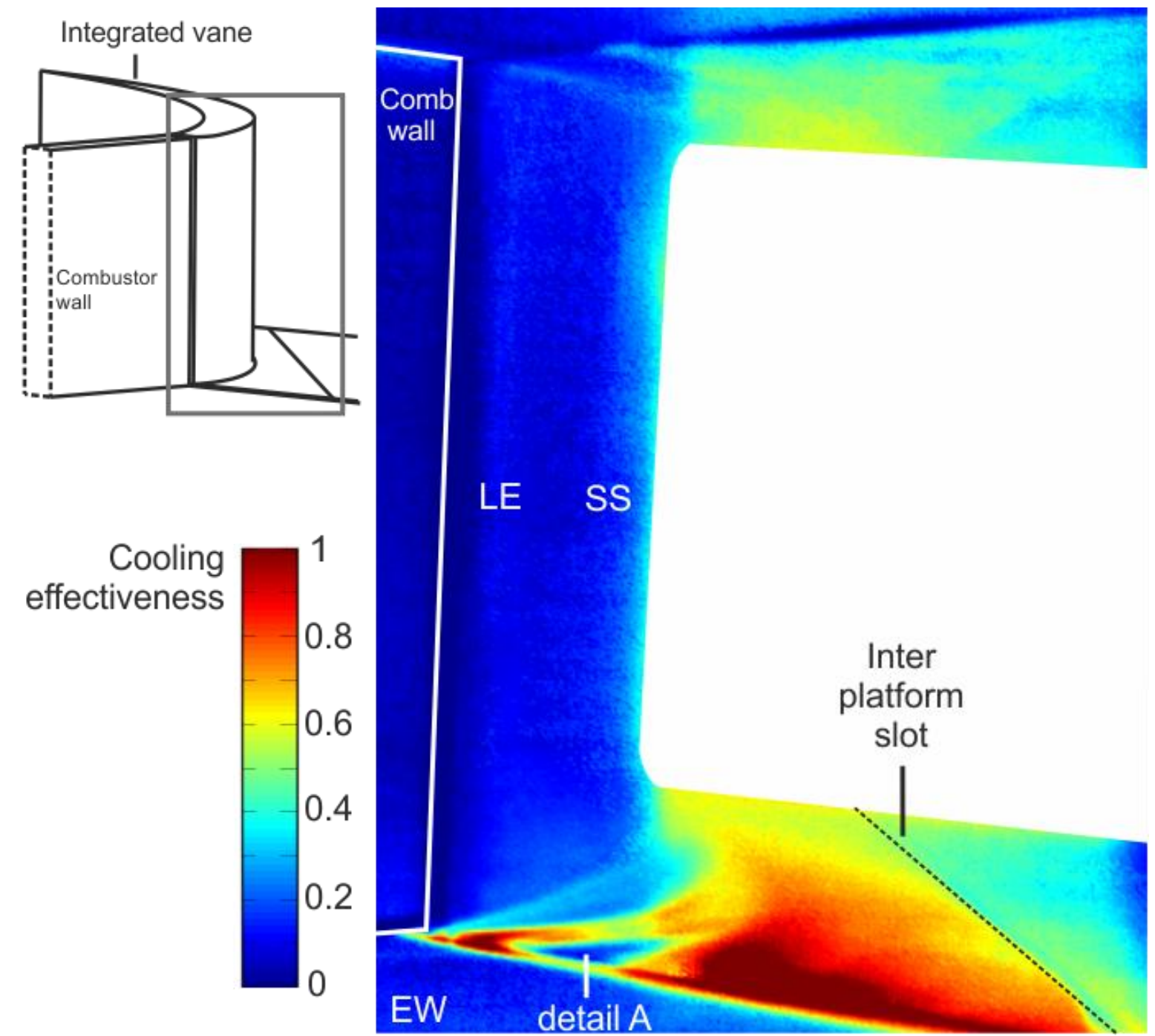

Figure 9-16: Measured cooling effectiveness on integrated vane hub endwall with endwall cooling 
A further view of the integrated vane with endwall cooling is needed to understand the convection of the coolant flow through the vane passage. Figure 9-17 thus shows the cooling effectiveness on the integrated vane's hub endwall, looking from downstream.

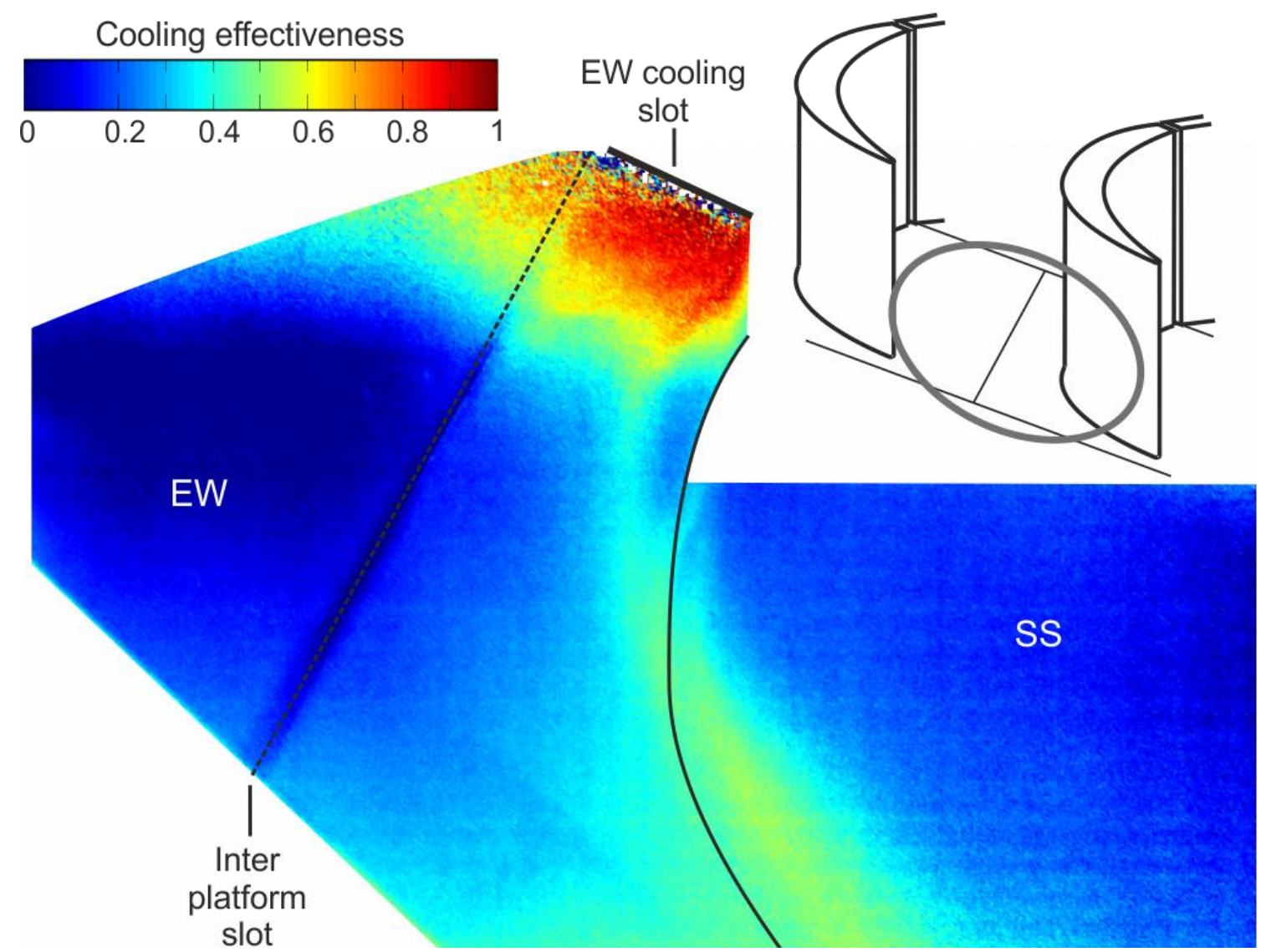

Figure 9-17: Measured cooling effectiveness on integrated vane hub endwall and suction surface with endwall cooling

The levels of cooling effectiveness at the endwall cooling slot agree well to the previous measurement in Figure 9-16. It can be seen that the majority of coolant flow is convected towards the integrated vane's suction side due to the pressure gradient in the passage. The coolant flow then migrates up onto the suction surface, following the path of the secondary flow. A further view is needed for a more detailed picture. Figure 9-18 thus shows the measured cooling effectiveness on the integrated vane's suction surface with endwall cooling. It can be seen that the coolant is convected onto the suction surface slightly further downstream than predicted by the CFD- 
simulations. Levels of cooling effectiveness decrease from peak values of 0.65 to 0.15 at the trailing edge. Qualitative and quantitative agreement of the experimental measurements with endwall cooling in Figure 9-16, Figure 9-17 and Figure 9-18 to the numerical prediction in Figure $9-13 \mathrm{~b}$ is found to be fairly good.

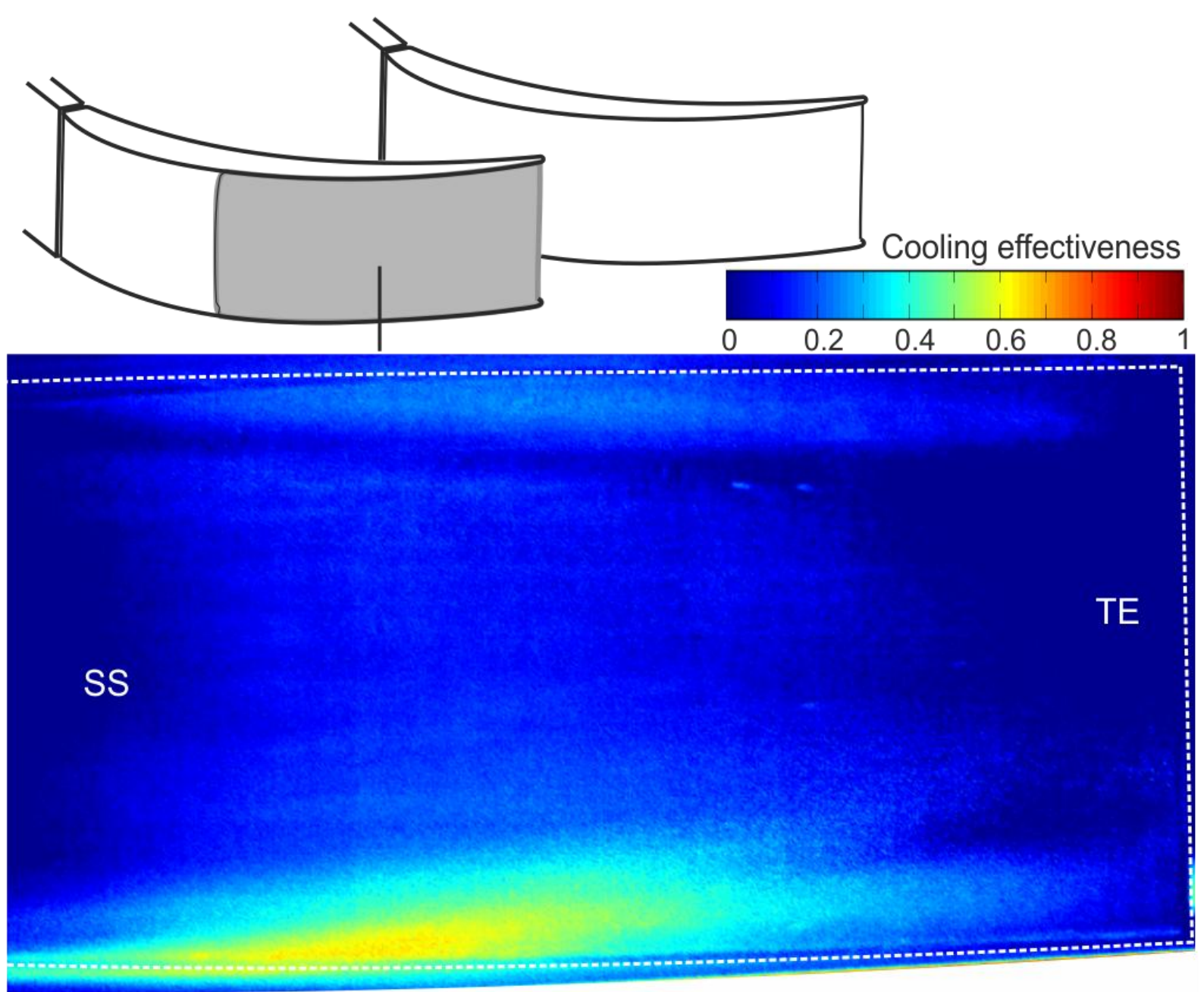

Figure 9-18: Measured cooling effectiveness on integrated vane suction surface with endwall cooling

Figure 9-12 and Figure 9-18 only show increased cooling effectiveness towards the hub of the suction surfaces and not towards the casing. This is because coolant flow is only ejected from the hub endwall in this experimental setup for simplicity reasons. In a realistic setup the distribution would be symmetrical with the same streak of cooling effectiveness seen on the suction surface towards the casing (as seen in the CFD-simulation). It is furthermore noted that the area that is cooled on the integrated 
vane suction surface when using only endwall cooling corresponds to the area that is uncooled when only using leading edge cooling. Leading edge and endwall cooling thus complement each other in the cooling effectiveness coverage of the suction surface.

In comparison to the hub endwall cooling effectiveness of the conventional vanes, the integrated vanes hub endwall coolant covers a wider area of the pitch. This can be better understood when looking at the static pressure distribution at the hub endwall in the location of the cooling slot. Figure 9-19 thus shows this static pressure (non-dimensionalised by the maximum static pressure) distribution along the pitch for conventional and integrated vane design.

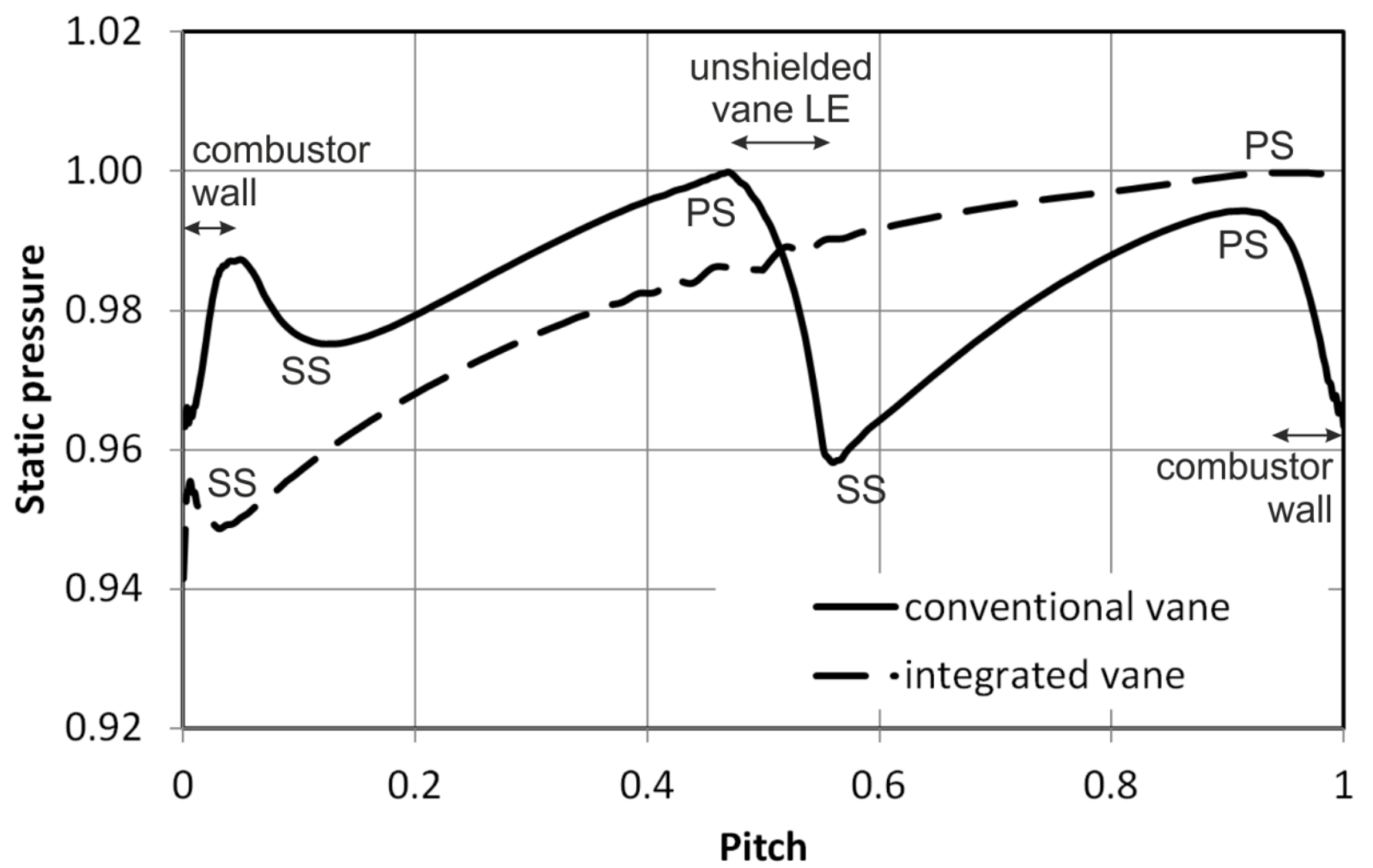

Figure 9-19: Dimensionless static pressure on hub endwall of conventional and integrated vane in the location of the cooling slot obtained from steady CFD simulations without cooling

It can be seen how the conventional vane's static pressure is low behind the combustor wall trailing edge and then rises across the first passage, reaching its 
maximum on the unshielded vane's pressure side. The pressure drops across the leading edge and again rises across the second passage, reaching its maximum at the shielded vane's pressure side. The integrated vane's static pressure shows a considerably simpler distribution due to the lack of a passage-central vane. It continuously rises across the pitch, from its minimum at the vane's suction side to its maximum on the vane's pressure side where it reaches exactly the same pressure as on the unshielded conventional vane's pressure side. In addition to the non-uniform static pressure distribution, the conventional vane design leads to the formation of a horse-shoe vortex system. It sweeps away the coolant from the endwall and is difficult to predict. As previously discussed, this horse-shoe vortex system formation is absent in the integrated vane design due to the lack of a vane stagnation pressure region. The integrated vane cooling thus not only covers a larger fraction of the endwall, it is also significantly easier to design.

\subsection{Numerical comparison of cooling effectiveness with realistic endwall cooling slot geometry}

The presented studies of endwall cooling were done using one simple inclined endwall cooling slot. This was done due to limitations of the manufacturing process (stereolithography could not make features of less than $0.5 \mathrm{~mm}$ ) and due to mechanical constraints (the thickness of the cooling box wall would not withstand the pressure within the plenum). Simulations were thus done using a more realistic endwall cooling geometry with two cooling slots. The new geometry, replacing the geometry seen in detail a) of Figure 9-1, is thus shown in Figure 9-20. Cooling flows 1 and 2 represent intended cooling flow and leakage flow respectively. Geometries and dimensions are representative of industrial practice. The figure also shows the 
mesh of the endwall cooling slots. The mesh size for conventional and integrated vane design is 6.6 million and 8.5 million respectively.

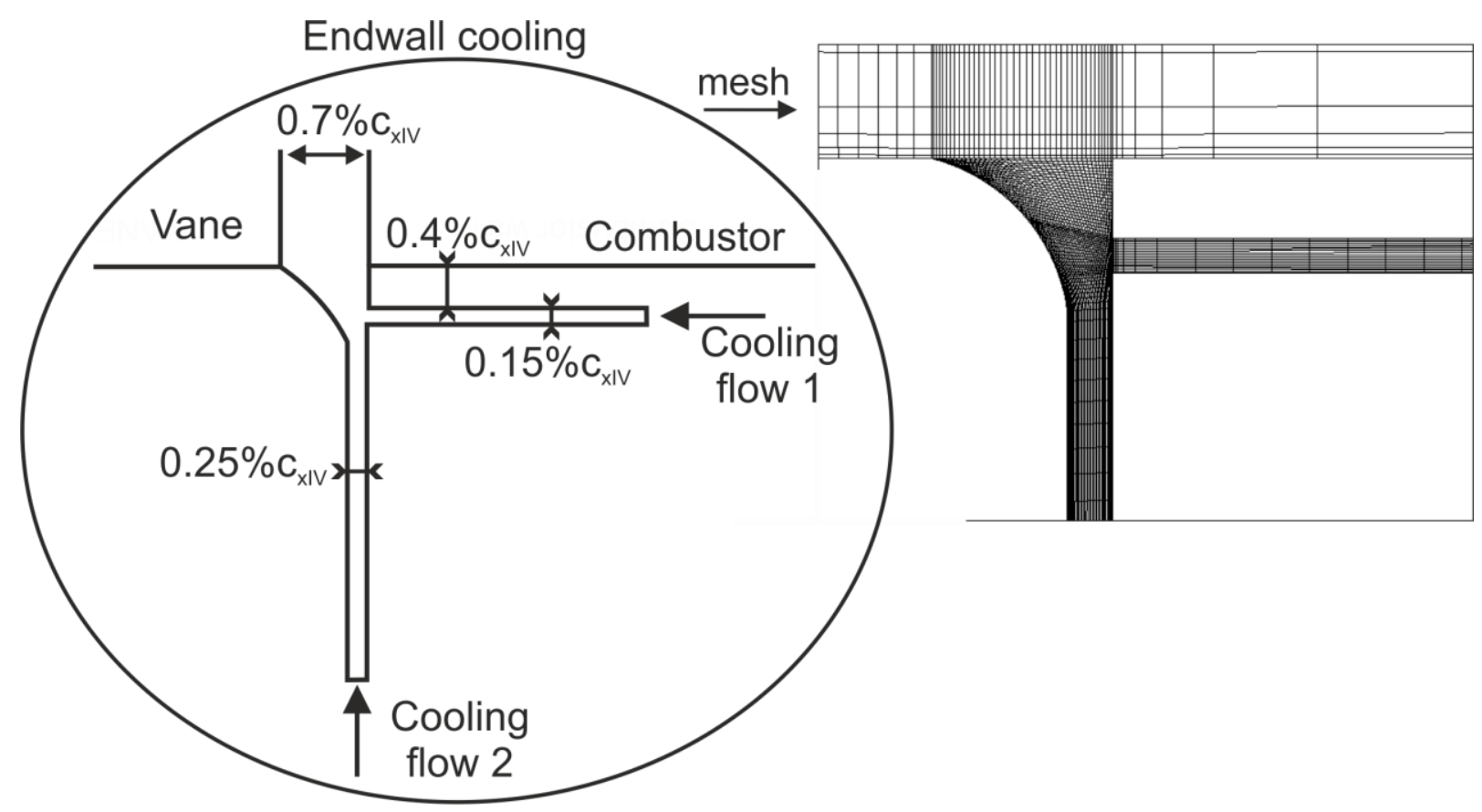

Figure 9-20: Schematic of realistic geometry and mesh of endwall cooling slots

Figure 9-21 shows the new predicted cooling effectiveness of conventional and integrated vane design. The inlet pressure of the endwall cooling slots was slightly increased to 1.59 bar. The cooling of the integrated vane endwalls $(0.9 \%$ coolant massflow are used for hub and casing endwall combined) is facilitated by the absence of a horseshoe vortex system and thus leads to a favourable, circumferentially more uniform surface coverage of cooling flow compared to the conventional vane design (which used $0.76 \%$ of endwall coolant massflow). Moreover, it can be seen that $0.57 \%$ of coolant massflow (in relation to mainstream massflow) from the transition duct wall suffice to effectively cool all integrated vane leading edges. This is especially relevant when considering that in a conventional design the coolant massflow emanating from the combustor wall is ejected into the mainstream without further purpose. Additionally, the entire cooling flow used for showerhead leading edge film cooling in the conventional vane design is saved and 
the total vane surface area is reduced by $10 \%$. This strengthens the authors' belief that ultimately the cooling flow of one conventional vane will be sufficient for one integrated vane, thus halving the amount of first vane cooling requirements.
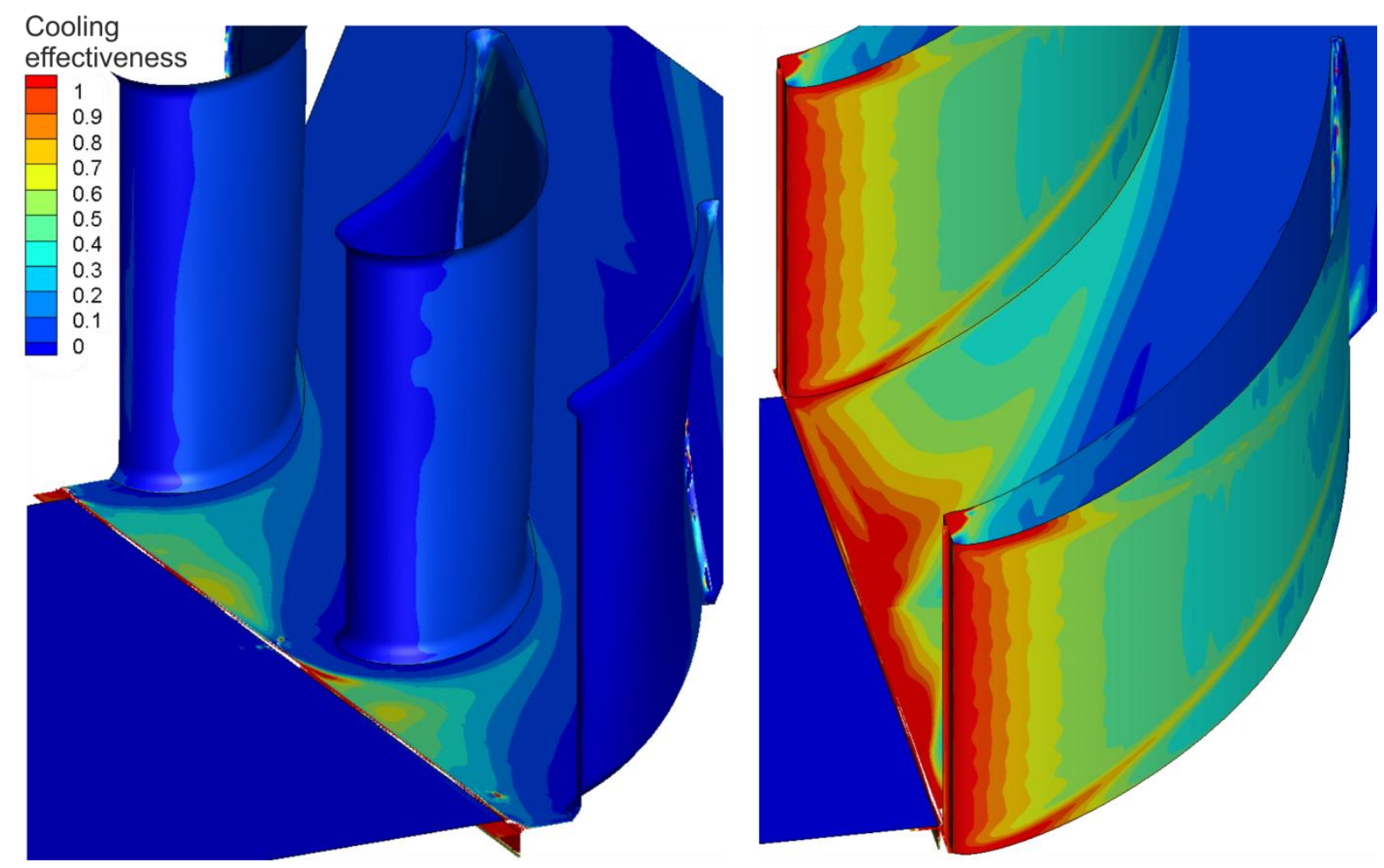

Figure 9-21: Predicted endwall cooling effectiveness of conventional vane (left), and endwall \& leading edge cooling effectiveness of integrated vane (right) 


\section{Chapter 10: Conclusion}

This thesis presented the development and aerothermal investigation of the integrated combustor vane concept, which replaces the high pressure turbines' nozzle guide vanes with vanes that extend the combustor walls. The integrated vanes were developed using the in-house CFD code TBLOCK with the goal of maintaining turning while reducing aerodynamic loss. Aerothermal experiments were conducted on a high-speed linear cascade comprising of two can combustors and either four conventional vanes or two integrated vanes. Aerodynamic measurements showed good agreement with the linear numerical simulations. A decrease in the total pressure loss coefficient of $27 \%$ at the exit of the stator row was illustrated, caused by a reduction in profile as well as endwall loss and a halving of the number of wakes.

Thermal measurements illustrated a significant reduction in the heat transfer coefficient for the integrated vane surfaces and endwalls in the order of $40 \%-50 \%$ compared to the conventional vanes. The reduction in the heat transfer coefficient on the integrated vane surfaces is caused by the close proximity of the integrated vane and combustor wall, which allows the combustor wall's boundary layer to transfer onto the vane. The extent of the heat transfer coefficient reduction is validated by a correlation for the heat transfer in a turbulent boundary layer on a flat plate. Moreover, the integrated vane concept leads to the removal of the horse-shoe vortex system and thus the region of high heat transfer coefficient values downstream of the passage vortex. The numerical simulations agree qualitatively and quantitatively well with the experimental measurements and support the physical explanations.

Annular full stage simulations confirm the validity of the linear aerothermal experimental and numerical results for realistic gas turbine applications. A gain in 
time-averaged stage efficiency of $1.5 \%$ is illustrated. This is attributed to the aerodynamic benefits within the stator and the superior aerodynamic performance within the rotor due to the halving of the number of wakes and the reduction of spanwise rotor incidence variation.

A further study illustrated the influence of engine realistic combustor flow with swirl on the integrated vane's aerothermal performance. The distributions of the heat transfer coefficient illustrate a slight, non-detrimental radial shift depending on the swirl orientation. The low pressure residual swirl core at the exit of the combustor transition duct slightly skews the integrated vane's wake and leads to a considerable spanwise variation in turning. This flow non-uniformity impacts the integrated vane's full-stage performance: The stage efficiency advantage over the conventional vane design drops to $0.7 \%$. It is nonetheless shown that profiling the integrated vane is suitable to homogenize the downstream flowfield and achieve further stage efficiency gains (of $0.23 \%$ for the chosen design).

A detailed cooling study shows good agreement between experiments and numerical predictions. The results illustrate a superior endwall coverage of cooling effectiveness due to a more uniform pitchwise pressure distribution and due to the removal of the horseshoe vortex system. Moreover, the potential of efficient cooling of the integrated vane leading edge and vane surfaces through cooling slots in the combustor wall is highlighted. The author believes that the cooling flow used for one conventional vane will be sufficient to cool one integrated vane, thus halving the amount of first vane cooling requirements.

Finally, by halving the number of vanes, simplifying the design and eliminating the need for leading edge film cooling, manufacturing and development costs are expected to be considerably reduced. 


\section{Suggestions for future work}

This thesis presented a detailed investigation of four key areas: Aerodynamics, heat transfer, stage efficiency and cooling. Nonetheless, several further studies are encouraged for an even better understanding of the concepts applicability.

Firstly it would be interesting to see the effect of the combustor flow's unsteadiness on the integrated vane's stage performance. While this study looked at unsteady stage performance, it used a constant (spatial and temporal) inlet boundary condition at the inlet of the domain (exit of the transition duct). It has been however shown that the precessing vortex core downstream of the combustor swirl generator is highly unsteady in nature with a significant impact on vane aerodynamics and heat transfer (Jacobi, Mazzoni, Chana, \& Rosic, 2017).

Secondly it would be important to consider the mechanical aspects of the integrated vane design. Since the integrated vanes have a reduced aspect ratio it would be relevant to know if the increased size poses difficulties for the manufacturing process (i.e. casting). Furthermore each vane's loading is essentially doubled and its thickness reduced. Would this limit its life span?

A further issue with the integrate vanes form, specifically its reduced thickness, is its cooling. Can the internal cooling architecture be implemented in the same manner as for conventional vanes. And does the reduced vane heat transfer and effective leading edge cooling geometry allow to (at least partially) remove external cooling. What would the effect of this be on the overall cycle performance?

A study should also look at the effect of combustion on the integrated vane's aerothermal performance, and how the acoustic modes are altered compared to a conventional vane design. What will be the impact on vibrations and rotor forcing? 
This brings us to the final point, costs. What does the increase in stage efficiency, the reduction in coolant requirements, the possible increases in turbine entry temperature due to improved heat transfer mean for the gas turbine's running costs (i.e. reduction in fuel consumption)? What are the potential savings associated to a reduction in development time, due to a simplification of the flowfield (i.e. box does the turning, and not vane passage) and the cooling design (i.e. improved endwall cooling coverage, removal of showerhead film cooling)? And what manufacturing cost reductions can be expected as a result of a halving of the number of first vane components and of an elimination of the need for drilling leading edge film cooling holes?

These are just a few aspects that could be considered for future work. Such investigations might just reveal further benefits of the concept, adding to the significant advantages highlighted in this work. 


\section{References}

Abuaf, N., Bunker, R., \& Lee, C. (1998). Effects of surface roughness on heat transfer and aerodynamic performance of turbine airfoils. ASME Journal of Turbomachinery, 120(3), pp. 522-529.

Airtx. (2016). Airtx.net. Retrieved from http://www.airtx.net/about-us

Alekseenko, S. V., Kuibin, P. A., \& Okulov, V. L. (2007). Theory of concentrated vortices. Berlin/Heidelberg: Springer.

Ames, F. (1997). The influence of large-scale high-intensity turbulence on vane heat transfer. ASME Journal of Turbomachinery, 119(1), pp. 23-30.

Ames, F. E. (1998). Aspects of Vane Film Cooling with High Turbulence- Part I: Heat Transfer. Journal of Turbomachinery, 120(4), 768-776.

Ames, F., Wang, C., \& Barbot, P. (2003). Measurement and Prediction of the Influence of Catalytic and Dry Low NOx Combustor Turbulence on Vane Surface Heat Transfer. ASME J. Turbomach., 125(2), pp. 221-231.

Anacleto, P. M., Fernandes, E. C., Heitor, M. V., \& Shtork, S. I. (2003). Swirl flow structure and flame characteristics in a model lean premixed combustor. Combustion Science and Technology, 175(8), pp. 1369-1388.

Aslanidou, I., Rosic, B., Knajirakkad, V., \& Uchida, S. (2011). Leading Edge Shielding Concept in Gas Turbines With Can Combustors. J. Turbomach 134(3), 031005.

Barringer, M. D., Richard, O. T., Walter, J. P., Stitzel, S. M., \& Thole, K. A. (2002). Flow field simulations of a gas turbine combustor. J. Turbomach 124(3), pp. 508-516.

Barringer, M., Thole, K., \& Polanka, M. (2004). Developing a Combustor Simulator for Investigating High Pressure Turbine Aerodynamics and Heat Transfer. ASME Turbo Expo GT2004-53613.

Barringer, M., Thole, K., \& Polanka, M. (2009). Effects of Combustor Exit Profiles on Vane Aerodynamic Loading and Heat Transfer in a High Pressure Turbine. Journal of Turbomachinery 131(2), 021008.

Beard, P., Smith, A., \& Povey, T. (2013). Effect of Combustor Swirl on Transonic High Pressure Turbine Efficiency. Journal of Turbomachinery 136(1), 011002.

Blair, M. F. (1974). An Experimental Study of Heat Transfer and Film Cooling on Large-Scale Turbine Endwalls. Journal of Heat Transfer 96(4), 524-529.

Bogard, D., \& Thole, K. (2006). Gas Turbine Film Cooling. Journal of Propulsion and Power, Vol. 22 (2), 249-270.

Bowman, C. (1992). Control of combustion-generated nitrogen oxide emissions: Technology driven by regulation. 24th Symposium (International) on Combustion, 24(1), (pp. 859878). 
Brennan, G., Harvey, N., Rose, M. G., Fomison, N., \& Taylor, M. (2003). Improving the efficiency of the trent $500 \mathrm{hp}$ turbine using non-axisymmetric end walls: Part 1: Turbine design. J. Turbomach. 125(3), pp. 497-504.

Came, P., \& Marsh, H. (1974). Secondary Flow in Cascades: Two simple derivations of the components of vorticity. Journal of Mechanical Engineering Science 16(6), 391-401.

Cameron, C., Brouwer, J., Wood, C., \& Samuelsen, G. (1989). A detailed characterization of the velocity and thermal fields in a model can combustor with wall jet injection. J. of Eng. for Gas Turbines and Power, 111(1), pp. 31-35.

Cha, C., Hong, S., Ireland, P., Denman, P., \& Savarianandam, V. (2012). Experimental and Numerical Investigation of Combustor-Turbine Interaction Using an Isothermal, Nonreacting Tracer. ASME J. Eng. Gas Turbines Power 134(8), p. 081501.

Cha, C., Hong, S., Ireland, P., Denman, P., \& Savarianandam, V. (2012). Turbulence Levels are High at the Combustor-Turbine Interface. ASME Paper No. GT2012-69130.

Correa, S. (1998). Power generation and aeropropulsion gas turbines: From combustion science to combustion technology. 27th Symposium (International) on Combustion/The Combustion Institute (pp. 1793-1807). Schenectady, NY 12301: GE Corporate Research \& Development Center.

Cranstone, A., Pullan, G., Curtis, E., \& Bather, S. (2014). Aerodynamic Design of High End Wall Angle Turbine Stages - Part I: Methodology Development. Journal of Turbomachinery, 136(2), 021006.

Cranstone, A., Pullan, G., Curtis, E., \& Bather, S. (2014). Aerodynamic Design of High End Wall Angle Turbine Stages: Part II: Experimental Verification. Journal of Turbomachinery 136(2), 021007.

Cutbirth, J. M., \& Bogard, D. G. (2002). Evaluation of Pressure Side Film Cooling with Flow and Thermal Field Measurements, Part I: Showerhead Effects. Journal of Turbomachinery, 124(4), 670-677.

Cutbirth, J. M., \& Bogard, D. G. (2002). Thermal Field and Flow Visualization Within the Stagnation Region of a Film Cooled Turbine Vane. Journal of Turbomachinery, 124(2), 200-206.

Dawson, J. R., Rodriquez-Martinez, V. M., Syred, N., \& O'Doherty, T. (2006). The effect of combustion instability on the structure of recirculation zones in confined swirling flames. Combust. Sci. and Tech., 177(12), pp. 2349-2371.

Denton, J. (1983). Denton, J. D., 1983, “An Improved Time-Marching Method for Turbomachinery Flow Calculation. J. Eng. Power, 105(3), pp. 514-521.

Denton, J. (1993). The 1993 IGTI Scholar Lecture: Loss Mechanisms in Turbomachines. ASME J. Turbomach., 115(4), pp. 621-656.

Denton, J. (1994). Designing in three dimensions. 
Denton, J., \& Pullan, G. (2012). A Numerical Investigation Into the Sources of Endwall Loss in Axial Flow Turbines. ASME Paper No. GT2012-69173.

Denton, J., \& Xu, L. (1999). The Exploitation of Three-Dimensional Flow in Turbomachinery Design. Proc. Inst. Mech. Eng., Part C: J. Mech. Eng. Sci., 213(2), pp. 125-137.

Doorly, J., \& Oldfield, M. (1987). The theory of advanced multi-layer thin film heat transfer gauges. International Journal of Heat Mass Transfer 30(6), 1159-1168.

Dunn, M. (1985). Turbine heat flux measurements: Influence of slot injection on vane trailing edge heat transfer and influence of rotor on vane heat transfer. J. Eng. Gas Turbines Power 107(1), pp. 76-83.

Ford, C., Carrotte, J., \& Walker, A. (2012). The Impact of Compressor Exit Conditions on Fuel Injector Flows. Jounral of Engineering for Gas Turbines and Power 134(11), 111504.

Friedrichs, S., Hodson, H. P., \& Dawes, W. N. (1996). Distribution of Film- Cooling Effectiveness on a Turbine Endwall Measured Using the Ammonia and Diazo Technique. Journal of Turbomachinery, 118(4), 613-621.

Friedrichs, S., Hodson, H. P., \& Dawes, W. N. (1997). Aerodynamic Aspects of Endwall FilmCooling. Journal of Turbomachinery, 119(4), 786-793.

Friedrichs, S., Hodson, H., \& Dawes, W. (1999). The design of an improved endwall film cooling configuration. Journal of Turbomachinery 121(4), 772-780.

Germain, T., Nagel, M., Raab, I., Schüpbach, P., Abhari, R., \& Rose, M. (2010). Improving Efficiency of a High Work Turbine Using Nonaxisymmetric Endwalls- Part I: Endwall Design and Performance. ASME Journal of Turbomachinery 132(2), 021007.

Gillespie, D. (1996). Intricate internal cooling systems for gas turbine blading. Oxford: University of Oxford, Engineering Science, DPhil thesis.

Goebel, S., Abuaf, N., Lovett, J., \& Lee, C. (1993). Measurements of combustor velocity and turbulence profiles. ASME Paper No. 93-GT-228.

Goldstein, R., \& Spores, R. (1988). Turbulent transport on the endwall in the region between adjacent turbine blades. ASME Journal of Heat Transfer, 110(4a), 862-869.

Granser, D., \& Schulenberg, T. (1990). Prediction and Measurement of Film Cooling Effectiveness for a First-Stage Turbine Vane Shroud. ASME Paper 90-GT-95.

Graziani, R., Blair, M., Taylor, J., \& Mayle, R. (1980). An Experimental Study of Endwall and Airfoil Surface Heat Transfer in a Large Scale Turbine Blade Cascade. Journal of Engineering and Power 102(2), 257-267.

Gregory-Smith, D., Graves, C., \& and Walsh, J. (1988). Growth of Secondary Losses and Vorticity in an Axial Turbine Cascade. ASME J. Turbomach. 110(1), pp. 1-8.

Griffini, D., Insinna, M., Salvadori, S., \& Martelli, F. (2015). Clocking Effects of Inlet NonUniformities in a Fully Cooled High-Pressure Vane: A Conjugate Heat Transfer Analysis. J. Turbomach 138(2), 021006. 
Hada, S., Yuri, M., Masada, J., Ito, E., \& Tsukagoshi, K. (2012). Evolution and Future Trend of Large Frame Gas Turbines: A New 1600 Degree C, J Class Gas Turbine. ASME Paper No. GT2012-68574.

Haldeman, C., Mathison, R., \& Dunn, M. (2004). Design, Construction and Operation of a Combustor Emulator for Short-Duration High-Pressure Turbine Experiments. 40th AIAA/ASME/SAE/ASEE Joint Propulsion Conference and Exhibit. American Institute of Aeronautics and Astronautics.

Hall, B., Chana, K., \& Povey, T. (2014). Design of a Nonreacting Combustor Simulator With Swirl and Temperature Distortion With Experimental Validation. J. Eng. Gas Turbines Power 136(8), 081501.

Han, J.-C., Dutta, S., \& and Ekkad, S. V. (2000). Gas Turbine Heat Transfer and Cooling Technology. Boca Raton, FL : CRC Press/Taylor \& Francis.

Han, S., \& Goldstein, R. (2006). Influence of blade leading edge geometry on turbine endwall heat (mass) transfer". ASME J. Turbomach. 128(4), pp. 798-813.

Hartland, J. C., Gregory-Smith, P. G., Harvey, N. W., \& Rose, M. G. (2000). Nonaxisymmetric turbine end wall design: Part ii experimental validation. ASME Journal of Turbomachinery, 122(2), 286-293.

Harvey, N. W., Rose, M. G., Taylor, M. D., Shahrokh, J., \& Gregory-Smith, D. G. (2000). Nonaxisymmetric Turbine End Wall Design: Part I - Three-Dimensional Linear Design System. ASME Journal of Turbomachinery, 122(2), 278-285.

Harvey, N., Brennan, G., Newman, D., \& Rose, M. (2002). Improving Turbine Efficiency Using Non-Axisymmetric End Walls: Validation in the Multi-Row Environment and With Low Aspect Ratio Blading. ASME Turbo Expo GT2002-30337. Amsterdam.

Huang, Y., \& Yang, V. (2009). Dynamics and stability of lean-premixed swirl-stabilized combustion. Progress in Energy and Combustion Science, 35(4), pp. 293 - 364.

Hurrion, J. (2002). The effect of an inlet temperature profile on the heat transfer to gas turbine blades. University of Oxford, Department of Engineering Science, DPhil thesis.

IEA. (2011). International Energy Agency - World Outlook. http://www.iea.org/.

Jacobi, S. (2013). Influence of Lean Premixed Combustor Geometry on the First Turbine Vanes' Aerothermal Performance. ETH Zurich; Department of Mechanical and Process Engineering.

Jacobi, S., \& Rosic, B. (2015). Development and Aerothermal Investigation of Integrated Combustor Vane Concept. ASME Journal of Turbomachinery 138(1), 011002.

Jacobi, S., \& Rosic, B. (2016). Thermal Investigation of Integrated Combustor Vane Concept under Engine-Realistic Conditions. ASME Journal of Turbomachinery 139(2), 02100510. 
Jacobi, S., \& Rosic, B. (2017). Full-Stage Performance of Integrated Combustor Vane Concept. Journal of Power and Propulsion Systems.

Jacobi, S., \& Rosic, B. (2018). Influence of Combustor Flow with Swirl on Integrated Combustor Vane Concept Full-Stage Performance. ASME Journal of Turbomachinery.

Jacobi, S., Mazzoni, C., Chana, K., \& Rosic, B. (2017). Investigation of Unsteady Flow Phenomena in First Vane Caused by Combustor Flow with Swirl. ASME Journal of Turbomachinery 139(4), 041006-12.

Jeong, J., \& Hussain, F. (1995). On the identification of a vortex. Journal of Fluid Mechanics, 285(2), 69-94.

Khanal, B., He, L., Northall, J., \& Adami, P. (2013). Analysis of Radial Migration of Hot-Streak in Swirling Flow Through High-Pressure Turbine Stage. ASME J. Turbomach., 135(4), p. 041005 .

Klostermeier, C. (2008). Investigation into the Capability of Large Eddy Simulation for Turbomachinery Design. PhD Thesis, Cambridge University Engineering Department.

Lakshminarayana, B. (1996). Fluid Dynamics and Heat Transfer of Turbomachinery. New York, USA: John Wiley \& Sons.

Langston, L. (1980). Crossflows in a turbine cascade passage. ASME Journal of Engineering for Power, 102(4), 866-874.

Lazik, W., Doerr, T., Bake, S., Bank, R., \& Rackwitz, L. (2008). Development of lean-burn low-nox combustion technology at rolls-royce deutschland. Preceedings of the ASME Turbo Expo GT2008-51115.

Lefebvre, A. (1999). Combustion: An International Series. In Gas Turbine Combustion. Taylor \& Francis.

Lefebvre, A., \& Ballal, D. (2010). Gas Turbine Combustion: Alternative Fuels and Emissions. CRC Press, Third Edition.

Lethander, A., Thole, K. A., Zess, G., \& Wagner, J. (2004). Optimizing the vane endwall junction to reduce adiabatic wall temperatures in a turbine vane passage. Journal of Propulsion and Power, Vol. 20, No. 6, pp. 1096-1104.

Lubbock, R. (2013). Fast insertion probes for unsteady temperature and turbulence measurements. DPhil Thesis, Department of Engineering Science, University of Oxford.

Luque, S., Kanjirakkad, V., Aslanidou, I., Lubbock, R., \& Rosic, B. (2015). A New Experimental Facility to Investigate Combustor-Turbine Interactions in Gas Turbines With Multiple Can Combustors. J. Eng. Gas Turbines Power 137(5), p.051503.

Mandai, S., Matsuura, M., Saitoh, K., Tanaka, K., \& Akizuki, W. (2004). Pilot burner, premixing combustor, and gas turbine US6701713 B2. Mitsubishi Heavy Industries, Ltd. 
Marn, A., Goettlich, E., Cadrecha, D., \& Pirker, H. (2009). Shorten the Intermediate Turbine Duct Length by Applying an Integrated Concept. J. Turbomach 131(4), 041014.

Mazzoni, C., Klostermeier, C., \& Rosic, B. (2014). Influence of Large Wake Disturbances Shed From the Combustor Wall on the Leading Edge Film Cooling. J. Eng. Gas Turbines Power 136(8), p.081503.

Mazzoni, C., Luque, S., \& and Rosic, B. (2015). Capabilities of Thermal Wall Functions to Predict Heat Transfer on the NGVS of a Gas Turbine With Multiple Can Combustors. ASME Paper No. GT2015-43515.

Mehendale, A. B., \& Han, J. C. (1992). Influence of High Mainstream Turbulence on Leading Edge Film Cooling Heat Transfer. Journal of Turbomachinery, Vol. 114(4), 707-715.

Moss, R. (1992). The effects of turbulence length scale on heat transfer. Ph.D. Dissertation, Department of Eng. Sc., University of Oxford, Report No. OUEL 1924/92.

Nealy, D., Mihelc, M., Hylton, L., \& and Gladden, H. (1984). Measurements of heat transfer distribution over the surfaces of highly loaded turbine nozzle guide vanes. ASME Journal of Engineering for Gas Turbines and Power, 106(1), 149-158.

O'Connor, J., \& Lieuwen, T. (2011). Further characterization of the disturbance field in a transversely excited swirl-stabilized flame. J. Eng. Gas Turbines Power 134(1), 011501.

Oke, R., Simon, T., Burd, S., \& Vahlberg, R. (2000). Measurements in a turbine cascade over a contoured endwall: discrete hole injection of bleed flow. ASME paper 2000-GT-214.

Oldfield, M. (2008). Impulse Response Processing of Transient Heat Transfer Gauge Signals. ASME J. Turbomach., 130(2), p. 021023.

Piccini, E. (1999). The development of a new heat transfer gauge for heat transfer facilities. Oxford: Univeristy of Oxford, Department of Engineering Science, DPhil Thesis.

Polanka, M. D., Witteveld, V. C., \& Bogard, D. G. (1999). Film Cooling Effectiveness in the Showerhead Region of a Gas Turbine Vane Part I: Stagnation Region and Near Pressure Side. ASME Paper 99-GT-048. American Society of Mechanical Engineers.

Povey, T., \& Qureshi, I. (2009). Developments in Hot-Streak Simulators for Turbine Testing. Journal of Turbomachinery 131(3), 031009.

Pullan, G., \& Harvey, N. (2007). Influence of Sweep on Axial Flow Turbine Aerodynamics at Midspan. Journal of Turbomachinery, 129(3), 591-598.

Pullan, G., \& Harvey, N. (2008). The Influence of Sweep on Axial Flow Turbine Aerodynamics in the Endwall Region. Journal of Turbomachinery, 130(4), 041011.

Pullan, G., Denton, J., \& Curtis, C. (2006). Improving the Performance of a Turbine with Low Aspect Ratio Stators by Aft-Loading. Journal of Turbomachinery, 128(3), 492-499.

Qureshi, I., Beretta, A., Chana, K., \& Povey, T. (2012). Effect of aggressive inlet swirl on heat transfer and aerodynamics in an unshrouded transonic hp turbine. J. Turbomach. 134(6), 061023. 
Qureshi, I., Smith, A. D., \& Povey, T. (2013). Hp vane aerodynamics and heat transfer in the presence of aggressive inlet swirl. J. Turbomach. 135(2), 021040.

Rahim, A., \& He, L. (2015). Rotor Blade Heat Transfer of High Pressure Turbine Stage Under Inlet Hot-Streak and Swirl. Journal of Engineering for Gas Turbines and Power, 137(6), 062601.

Reiss, H., \& Bolcs, A. (2000). Experimental Study of Showerhead Cooling on a Cylinder Comparing Several Configurations Using Cylindrical and Shaped Holes. Journal of Turbomachinery, Vol. 122(1), 161-169.

Reynolds, W., Kays, W., \& Kline, S. (1958). Heat transfer in a turbulent incompressible boundary layer. $i$ : Constant wall temperature. NASA Memo 12-1-58W.

Richards, A. (2015). University of oxford advanced research computing facility. Retrieved from dx.doi.org/10.5281/zenodo.22558

Roach, P. (1987). The Generation of Nearly Isotropic Turbulence by Means of Grids. Int. J. Heat Fluid Flow, 8(2), pp. 82-92.

Rolls-Royce. (2015). The Jet Engine, 5th edition. Derby: Wiley-Blackwell.

Rose, M. G., Harvey, N. W., Seaman, P., Newman, D. A., \& McManus, D. (2001). Improving the efficiency of the trent $500 \mathrm{hp}$ turbine using non-axisymmetric end walls: Part 2: Experimental validation. ASME 2001-GT-0505.

Rosic, B., Denton, J., Horlock, J., \& Uchida, S. (2011). Integrated Combustor and Vane Concept in Gas Turbines. ASME J. Turbomach. 134(3), p.031005.

Roux, S., Lartigue, G., \& Poinsot, T. (2005). Studies of mean and unsteady flow in a swirled combustor using experiments, acoustic analysis, and large eddy simulations. Combustion and Flame, 141(12), pp. 40-54.

Salvadori, S., Riccio, G., Insinna, M., \& Martelli, F. (2012). Analysis of Combustor/Vane Interaction With Decoupled and Loosely Coupled Approaches. ASME Paper No. GT2012-69038.

Schildmacher, K. U., \& Koch, R. (2005). Experimental investigation of the interaction of unsteady flow with combustion. J. Eng. Gas Turbines Power 127(2), 295-300.

Schluter, J., Schonfeld, T., Poinsot, T., Krebs, W., \& Hoffman, S. (2001). Characteristics of confined swirl flows using large eddy simulations. ASME Turbo Expo 2001GT-0060.

Schmid, G., Krichbaum, A., Werschnik, H., \& Schiffer, H. (2014). The Impact of Realistic Inlet Swirl in a 1.5 Stage Axial Turbine. ASME Paper No. GT2014-26716.

Schüpbach, P., Abhari, R., Rose, M., Germain, T., Raab, I., \& Gier, J. (2010). Improving Efficiency of a High Work Turbine Using Nonaxisymmetric Endwalls_Part II: TimeResolved Flow Physics. ASME Journal of Turbomachinery 132(2), 021008.

Selle, L., Benoit, L., Poinsot, T., Nicoud, F., \& Krebs, W. (2006). Joint use of compressible large eddy simulation and helmholtz solvers for the analysis of rotating modes in a industrial swirled burner. Combustion and Flame, 145(1-2), pp. 194-205. 
Selle, L., Lartigue, G., Poinsot, T., Koch, R., Schildmacher, K. U., \& Krebs, W. (2004). Compressible large eddy simulation of turbulent combustion in complex geometry on unstructured meshes. Combustion and Flame, 137(4), 489-505.

SHARCNET. (n.d.). Lambda 2 definition. Retrieved from https://www.sharcnet.ca/Software/Ansys/16.2.3/en-us/help/cfd_post/vortcore_details_v.html

Sharma, O., \& Butler, T. (1987). Predictions of endwall losses and secondary flows in axial flow turbine cascades. ASME Journal of Turbomachinery, 109(2), 229-236.

Shih, T. I.-P., \& Lin, Y.-L. (2003). Controlling secondary-flow structure by leading-edge airfoil fillet and inlet swirl to reduce aerodynamic loss and surface heat transfer. ASME J. Turbomach 125(1), pp. 48-56.

Sieverding, C. (1985). Recent Progress in the Understanding of Basic Aspects of Secondary Flows in Turbine Blade Passages. ASME J. Eng. Gas Turbines Power 107(2), pp. 248-257.

Syred, N. (2006). A review of oscillation mechanisms and the role of the precessing vortex core (pvc) in swirl combustion systems. Progress in Energy and Combustion Science 32(2), 93-161.

Takahashi, R., \& Ni, R. (1990). Unsteady euler analysis of the redistribution of an inlet temperature distortion in a turbine. AIAA Paper No. 90-2262.

Thole, K. (2006). Airfoil Endwall Heat Transfer. In The Gas Turbine Handbook. DOE, Morgantown, WV.: National Energy Technology Laboratory.

Thole, K., \& Knost, D. (2005). Heat transfer and film-cooling for the endwall of a first stage turbine vane. International Journal of Heat and Mass Transfer 48(25-26), 5255-5269.

Turrell, M. D., Stopford, P. J., Syed, K., \& Buchanan, E. (2004). Cfd simulations of the flow within and downstream of high swirl lean premixed gas turbine combustors. Proceedings of ASME Turbo Expo GT2004-53112.

Wang, D., \& He, L. (2010). Adjoint Aerodynamic Design Optimization for Blades in Multistage Turbomachines - Part I: Methodology and Verification. ASME Journal of Turbomachinery 132(2), 021011.

Wang, D., He, L., \& Wells, R. (2010). Adjoint Aerodynamic Design Optimization for Blades in Multistage Turbomachines - Part II: Validation and Application. Journal of Turbomachinery 312(2), 021012.

Wang, P., Bai, X. S., \& Wessman, M. (2004). Large eddy simulation and experimental studies of a confined turbulent swirling flow. Physics of Fluids, 16(9), pp. 3306-3324.

Wang, S., Yang, V. H., Hsieh, S., \& Mongiah, C. (2007). Large eddy simulations of gasturbine swirl injector flow dynamics. J Fluid Mech, 583, pp. 99-122. 
Wankhede, M. J., Bressloff, N. W., Keane, A. J., Caracciolo, L., \& Zedda, M. (2010). An analysis of unstable flow dynamics and flashback mechanism inside a swirlstabilised lean burn combustor. Proceedings of ASME Turbo Expo GT2010-22253.

Weisstein, E. (n.d.). Error function (erfc). Retrieved from From MathWorld--A Wolfram Web Resource: http://mathworld.wolfram.com/Erfc.html

Witteveld, V. C., Polanka, M. D., \& Bogard, D. G. (1999). Film Cooling Effectiveness in the Showerhead Region of a Gas Turbine Vane Part II: Stagnation Region and Near Suction Side. ASME Paper 99-GT-049. American Society of Mechanical Engineers.

Xiyun, L., Wang, S., Sung, H., Hsieh, S., \& Yang, V. (2005). Large eddy simulations of turbulent swirling flows injected into a dump chamber. Journal of Fluid Mechanics, 527, pp. 171-195.

Yoon, S., Denton, J., Curtis, E., Longley, J., \& Pullan, G. (2014). Improving Intermediate Pressure Turbine Performance by Using a Nonorthogonal Stator. Journal of Turbomachinery, 136(2), 021012.

You, D., Ham, F., \& Moin, P. (2008). Large-eddy simulation analysis of turbulent combustion in a gas turbine engine combustor. Center for Turbulence Research; Annual Research Briefs 2008.

Zess, G., \& Thole, K. (2002). Computational Design and Experimental Evaluation of Using a Leading Edge Fillet on a Gas Turbine Vane. Journal of Turbomachinery 124(2), 167175.

Zimmerman, D. R. (1979). Laser anemometer measurements at the exit of a t63-c20 combustor. NASA, Contract NAS 3-21267. 